\title{
In-Cylinder Fuel and Lubricant Effects on Gasoline Engine Friction
}

\section{Oliver Mark Edward Smith \\ (MEng)}

\author{
Submitted in accordance with the requirements for the degree of \\ Doctor of Philosophy \\ The University of Leeds \\ School of Mechanical Engineering
}

March 2007

The candidate confirms that the work submitted is his own and that appropriate credit has been given where reference has been made to the work of others.

This copy has been supplied on the understanding that it is copyright material and that no quotation from the thesis may be published without proper acknowledgement. 
For Sarah 


\section{Acknowledgements}

I wish to express my sincere gratitude to Prof. Martin Priest for his dedicated contribution to the supervision of this project, his knowledgeable counsel provided an invaluable resource throughout the research. I feel very fortunate to have been guided by such a gifted individual and can only hope that our time working together has imparted some of his vast tribological knowledge to me.

I would like to thank the Engineering and Physical Science Research Council and Shell Global Solutions (UK) for sponsoring this research, the latter also for providing technical assistance. In particular, Dr Ian Taylor (Shell) for his help and advice throughout the project. It is also necessary to note the invaluable contribution of Dr Richard Price, Dr Alex Cantlay, Dr Nigel Tait and Dr Catherine Boyd and Dr Hugues Renondeau to the success of this research.

I am particularly grateful to several colleagues at the University of Leeds for their contribution to this study. Prof. Dick Coy for the time he spent with me discussing all aspects of the project and Prof. Malcolm Fox whose enthusiasm and skill of disseminating his knowledge of complex chemistry is quite unique. I wish also to acknowledge the valuable guidance and friendship of Dr Dave Barrell whose input was significant throughout my work.

Technical staff at the University of Leeds contributed greatly to this study. Mr Paul Banks and Mr Keith Norris are both worthy of particular note as they provided essential practical help throughout the experiments which populate this thesis.

Throughout my academic career I have been supported by my parents whom I wish to acknowledge for both their financial backing and unfaltering belief in me, without which this study would not have been possible. 


\section{Abstract}

The purpose of the research reported in this thesis was to investigate the viability and quantify the potential gains of improving fuel economy of the gasoline engine through strategic application of additives.

An increased awareness of the link between greenhouse gas emissions and global warming means that there is a desire to reduce carbon dioxide emissions from transportation. There is therefore a growing emphasis on improving the fuel economy performance of vehicles. The addition of friction modifier additives to the fuel is one way to achieve this.

Using bespoke in-cylinder sampling techniques, an understanding of the operation of the piston assembly, a system responsible for much of the power loss in the internal combustion engine, is developed. Validation is given to the hypothesis that fuel economy gains can be achieved through the application of friction modifier administered to the engine via the gasoline. Results show gasoline administered friction modifier additive can accumulate in the piston assembly lubricant at levels 77 times greater than the initial fuel treatment level.

The performance of a large number of friction modifier additives were uniquely screened in a novel bench-top test which simulated the arduous in-cylinder conditions found in a firing gasoline engine. The test generated vast amounts of information which led to high performance formulations capable of reducing the friction coefficient in both the boundary and mixed lubrication regimes by around $50 \%$ when compared with the result for the base oil alone. Surface analysis techniques were also employed on engineering surfaces coated with friction modifier additives and add to the knowledge of their mechanism of action.

Finally a series of engine tests were conducted which prove the effectiveness of friction modifier administered to the engine via the gasoline. A fuel economy improvement of approximately $2 \%$ was seen where friction modifier gasoline was employed. Application of successful technology such as this is shown to correspond to the projected saving of around 502 million litres of gasoline and 388,000 tonnes of carbon $\left(\mathrm{CO}_{2}\right)$ per year in the UK alone. 


\section{Contents}

Acknowledgements............................................................................................ iii

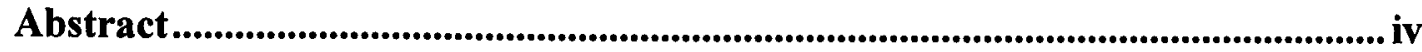

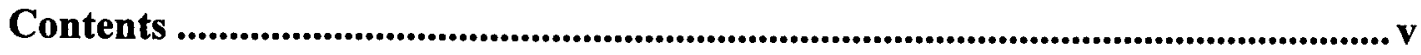

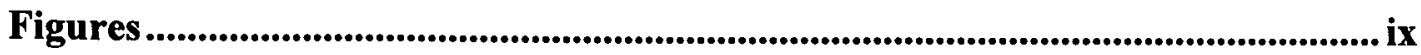

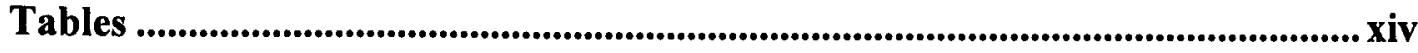

Abbreviations .................................................................................................... xvi

Notation .......................................................................................................... xviii

1. Introduction ...................................................................................................... 1

1.1. Engine Friction and Lubrication ..................................................... 4

1.2. Engine Lubricants and Formulation .................................................... 7

1.3. Lubricant Formulations for Fuel Economy ........................................ 11

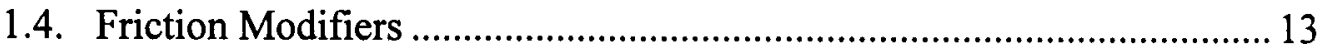

1.5. Gasoline and Gasoline Additives ................................................... 18

1.6. Fuel and Lubricant Interactions............................................................ 21

1.7. Other Attempts at Increasing Fuel Economy ....................................... 25

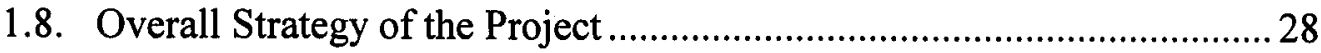

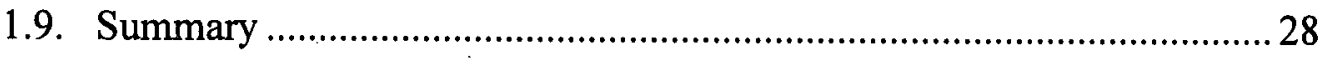

2. In-Cylinder Lubricant Sampling ............................................................ 30

2.1. The Ricardo Hydra Gasoline Engine................................................. 30

2.2. Top Ring Zone Sampling ..................................................................... 32

2.2.1. Top Ring Zone Sampling Tests......................................... 33

2.3. Three Point Lubricant Sampling From The Cylinder Wall (TPS) .......... 34

2.3.1. Development and Problems with the TPS System................ 37

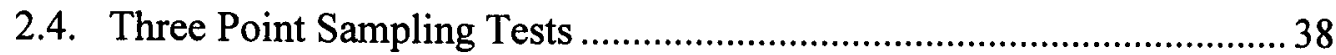

2.4.1. Cold-Start Engine Tests .................................................. 38

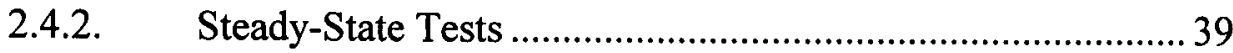

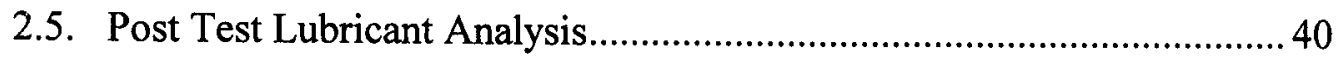

2.5.1. Fuel Dilution Measurement by Gas Chromatography........... 41

2.5.2. Potassium Concentration Testing by ICP-AES Analysis ...... 41

2.5.3. Dynamic Viscosity Measurement ....................................... 41 
2.6. Top Ring Zone Sampling Results and Analysis................................... 43

2.7. Three Point Sampling Results and Analysis .......................................... 47

2.7.1. Cold-Start TPS Results and Analysis.................................4 47

2.7.2. Steady-State TPS Results and Analysis .............................. 49

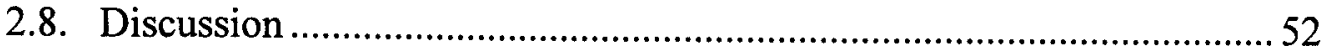

2.8.1. TRZ Sampling Discussion ...............................................52

2.8.2. Three Point Sampling Discussion ....................................... 54

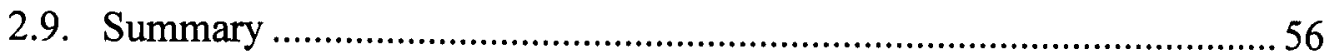

3. Tribometer Investigation of Environmentally Friendly Friction Modifier Additives ....................................................................................................59

3.1. The Cameron Plint TE-77 Machine and Test Specimens ......................6 60

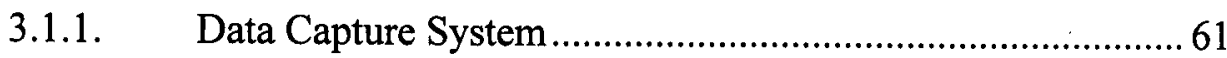

3.1.2. Reciprocator Rig Components .........................................6 62

3.2. Organic Friction Modifiers Under Investigation....................................64 64

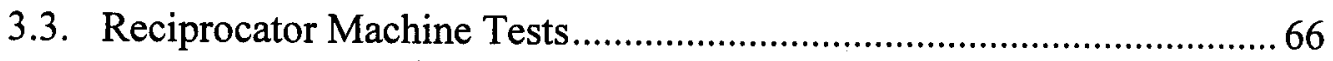

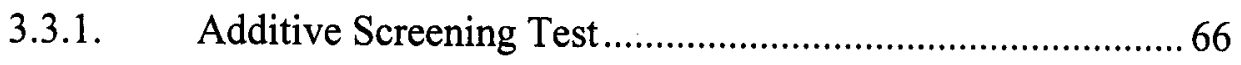

3.3.2. Additive Package Formulation and Blending Test .............. 69

3.3.3. Additive Concentration Tests.............................................69 69

3.3.4. Piston Ring Type Test ...................................................... 70

3.3.5. Film Creation Tests ........................................................ 71

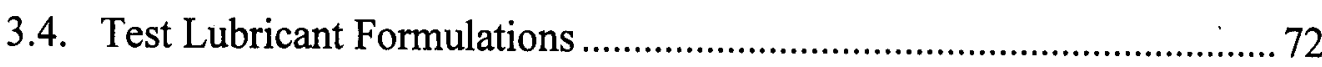

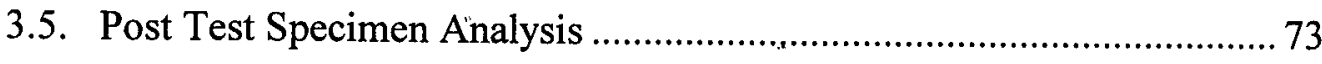

3.5.1. Fourier Transform InfraRed Microscopy (FTIR) ................. 73

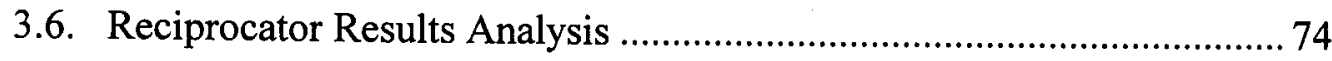

3.6.1. Additive Screening Tests Results Analysis......................... 74

3.6.2. Additive Package Formulation and Blending Results

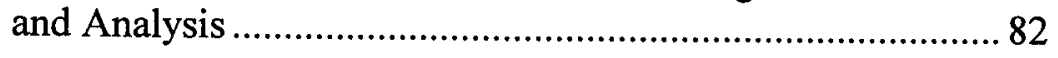

3.6.3. Additive Concentration Investigation Results and Analysis. 83

3.6.4. Piston Ring Type Tests Results and Analysis ..................... 84

3.6.5. Film Creation FTIR Microscopy Results and Analysis .......87

3.7. Reciprocator Machine Tests Discussion ............................................. 94

3.7.1. Additive Screening Tests Discussion.................................. 94 
3.7.2. Additive Concentration Tests Discussion ............................... 98

3.7.3. Piston Ring Material Friction Discussion ............................ 100

3.8. Film Creation FTIR Microscopy Discussion ......................................... 101

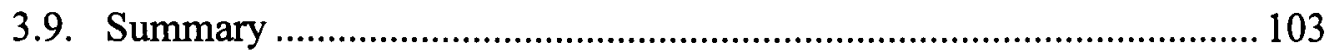

4. Mini Traction Machine Investigation of Novel Friction Modifier

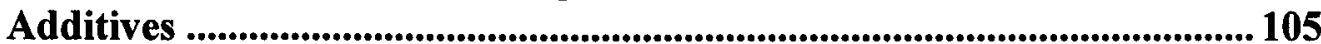

4.1. The Mini Traction Machine and Test Specimens ................................. 105

4.1.1. Mini Traction Machine Components .................................... 106

4.2. Post Test Specimen Analyses................................................................. 107

4.2.1. Optical Microscopy …………………………………........... 109

4.2.2. Form Talysurf Contacting Profilometer Measurement ....... 109

4.2.3. Scanning Electron Microscopy and Energy Dispersive X-ray Technique (SEM / EDX).............................................. 110

4.2.4. X-ray Photon Spectroscopy (XPS)...................................... 111

4.2.5. Fourier Transform InfraRed Microscopy (FTIR)................. 111

4.3. Mini Traction Machine Test................................................................ 112

4.4. Test Lubricant Formulations ................................................................... 114

4.5. MTM Friction Coefficient Measurement Results and Analysis ............ 115

4.6. Optical Microscopy Results and Analysis............................................ 119

4.7. Profilometry Results and Analysis ...................................................... 121

4.8. SEM / EDX Results and Analysis........................................................ 123

4.9. XPS Results and Analysis ................................................................... 125

4.10. FTIR Microscopy Results and Analysis................................................ 128

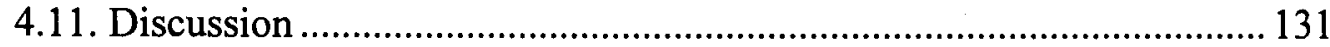

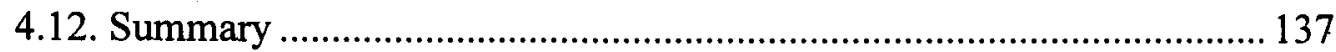

5. Novel Fuel Formulation Engine Tests ........................................................... 140

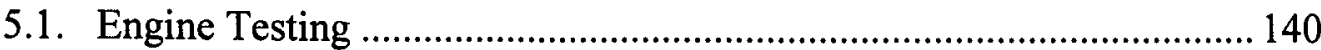

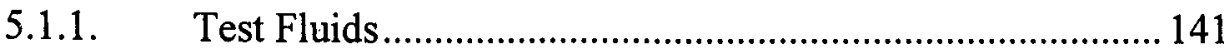

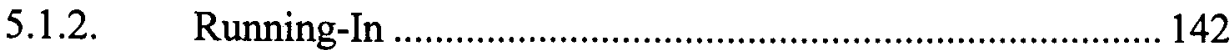

5.1.3. Engine Performance Testing ............................................. 142

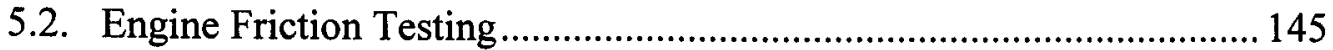

5.2.1. Standard Engine Friction Test Methodology ....................... 148

5.2.2. Modified Engine Friction Test Methodology ....................... 150 


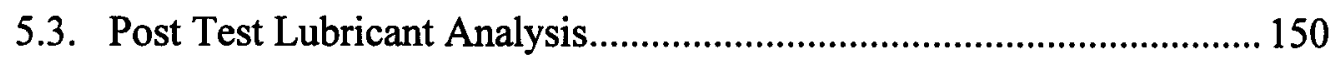

5.3.1. Fourier Transform InfraRed Microscopy (FTIR)................. 150

5.3.2. Fuel Dilution Measurement by Gas Chromatography......... 151

5.4. Engine Performance Tests Results and Analysis .................................. 151

5.5. Engine Friction Tests Results and Analysis ........................................ 156

5.6. Lubricant Analysis Results and Analysis ............................................... 161

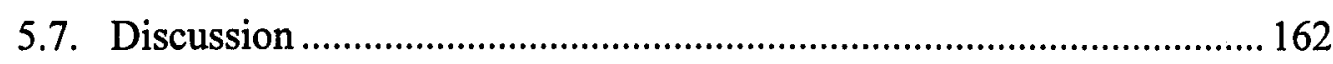

5.7.1. Engine Performance Tests Discussion ................................. 162

5.7.2. Engine Friction Tests Discussion........................................ 164

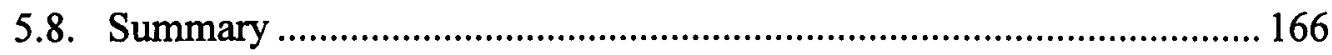

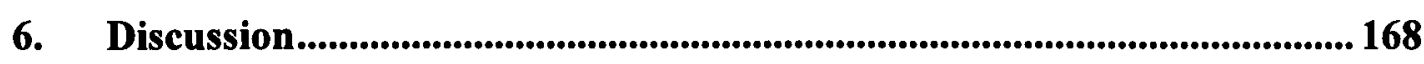

6.1. Key Project Findings ............................................................................ 168

6.1.1. The Success of FM Gasoline............................................... 178

6.2. The Global Impact Of The Research..................................................... 180

7. Conclusions \& Recommendations for Future Work ..................................... 183

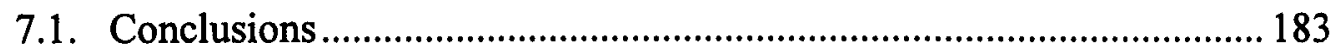

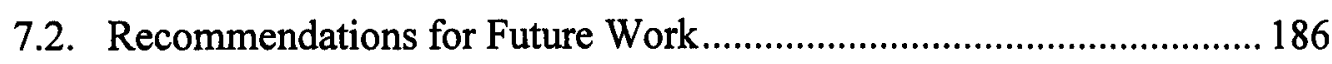

7.2.1. Further Work ...................................................................... 187

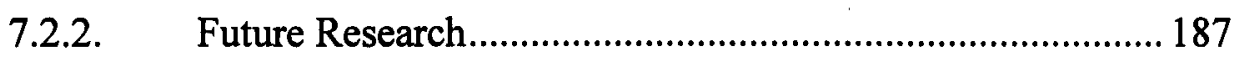

References ....................................................................................................................................... 189

Appendix ................................................................................................................................. 209

A1: Determination of Normal Force Present At the Cylinder Liner / Piston Ring Interface:............................................................................. 209

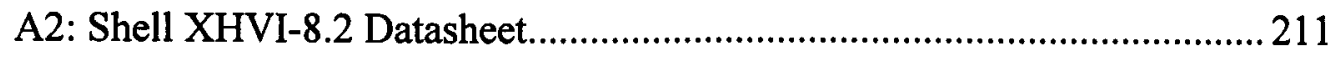

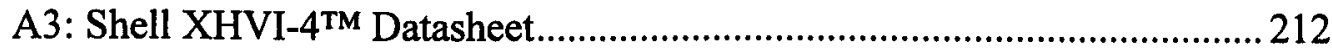

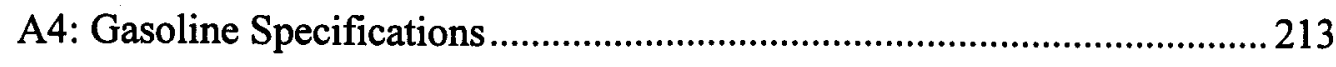

A5: TPS System Manufacturing Information .............................................. 214 


\section{Figures}

Figure 1-1 Global Information Flows Governing Legislation ................................ 2

Figure 1-2 Automotive Lubricant Legislation (Bardasz 2005) .............................. 3

Figure 1-3 Typical Fuel Energy Distribution in an Internal Combustion Engine. After (Taylor 1998)........................................................5

Figure 1-4 Typical Four-Stroke Gasoline Engine Cylinder Layout and Piston Assembly. After (Priest 1996; Subaru 2006) ...................................... 5

Figure 1-5 Modified Stribeck Diagram (Priest 2000) .......................................... 7

Figure 1-6 Sulphated Ash Blockage in Catalyst (Bardasz 2005) ........................... 10

Figure 1-7 Fatty Acid Formation on Ideal Surfaces ........................................... 14

Figure 1-8 Breakdown or Transition Temperature of Fatty Acids on Steel Surfaces (Bowden and Tabor 1950) ................................................... 15

Figure 1-9 Measured Boundary Coefficient of Friction of Fatty Acids (Castle and Bovington 2003)

Figure 1-10 Measured Boundary Coefficient of Friction Modifiers (Castle and Bovington 2003)

Figure 1-11 Patenting of Different Types of Fuel Additives Between 1981 and 2000: 1) detergents; 2) depressants and wax dispersants; 3) antioxidants, metal deactivators, stabilisers; 4) ignition modifier; 5) combustion modifiers; 6) other. (Danilov 2001).

Figure 1-12 Image of Fuel Dilution in the Cylinder Liner Oil Film. After (Kim, Yoon et al. 2003).

Figure 1-13 'Gel-Like' Sludge Formation in Engine Sump as a Result of Incompatibility Between Lubricant and Fuel Additive (Thiel and Hayden 2001b)

Figure 2-1 The Ricardo Hydra Research Engine ................................................ 31

Figure 2-2 Top Ring Zone Sampling Apparatus ................................................. 32

Figure 2-3 CAD Image of Three Point Sampling System...................................... 35

Figure 2-4 Three Point Sampling System: (a) TDC Reversal, (b) BDC Reversal................................................................................ 35

Figure 2-5 Flow Diagram of Three Point Sampling System .................................. 36

Figure 2-6 Photograph of TPS Liner, Sampling Pipe Attachment and Carbon Deposit

Figure 2-7 Bohlin CV120HR Dynamic Viscometer. 
Figure 2-8 Sump Sample Viscosity Analysis Repeatability. 43

Figure 2-9 Top Ring Zone Fuel Dilution Variation with Engine Condition. 44

Figure 2-10 Variation of Potassium Concentration with Varying Engine Load ...... 44

Figure 2-11 Variation of Potassium Concentration with Varying Engine Speed .... 45

Figure 2-12 Variation of Potassium Concentration with Varying Lambda Ratio

Figure 2-13 Variation of TRZ Potassium Concentration with Varying Fuel Potassium Concentration.

Figure 2-14 Variation of Potassium Concentration with Different Boiling Point Fuels.

Figure 2-15 Cold-Start Sump Lubricant Sample Fuel Dilution and Dynamic Viscosity Variation with Time.

Figure 2-16 Fuel Dilution in Both TPS and Sump Samples for Fuel Ab. 48

Figure 2-17 Fuel Dilution in Both TPS and Sump Samples for Fuel Ba

Figure 2-18 Top Sample Fuel Dilution (a) and Potassium Concentration (b) with Varying Engine Condition

Figure 2-19 Middle Sample Fuel Dilution (a) and Potassium Concentration

(b) with Varying Engine Condition.

Figure 2-20 Bottom Sample Fuel Dilution (a) and Potassium Concentration

(b) with Varying Engine Condition. .50

Figure 2-21 Dynamic Viscosity Variation with Engine Speed and Sample Location for Half Load Condition.

Figure 2-22 Dynamic Viscosity Variation with Engine Speed and Sample Location for Full Load Condition

Figure 2-23 Photograph of Samples Taken From the Bottom, Middle and Locations on the Cylinder Liner. .52

Figure 3-1 Plint TE-77 High Friction Reciprocator Tribometer 60

Figure 3-2 Schematic Representation of Loaded Piston Ring Section on Cylinder Liner Specimen in TE-77.

Figure 3-3 Schematic Representation of Reciprocator Setup 66

Figure 3-4 Photograph of Chrome Coated and Molybdenum Coated Piston Rings 70

Figure 3-5 Additive Screening Test Coefficient of Friction Results. .76

Figure 3-6 Reduction in Coefficient of Friction................................................... 77

Figure 3-7 Performance Related to Saturation of Chemical Bonds .77

Figure 3-8 Pure Base Oil Instantaneous Friction and Contact Potential Measurement. 
Figure 3-9 Fatty Acid Instantaneous Friction and Contact Potential

Measurement 79

Figure 3-10 Amide Instantaneous Friction and Contact Potential Measurement .... 80

Figure 3-11 Triglyceride and Ester Instantaneous Friction and Contact Potential Measurement. .81

Figure 3-12 Additive Blending Results............................................................ 82

Figure 3-13 Additive Concentration Effects ..................................................... 83

Figure 3-14 Piston Ring Material Coefficient of Friction Results .......................... 85

Figure 3-15 Instantaneous Friction and Contact Potential Results (Mo Ring) ........ 86

Figure 3-16 Film Conductivity Test Conducted on Stearic Acid...........................87

Figure 3-17 FTIR Analysis of Chrome Ring (a) and Cylinder Liner (b) Run with Linoleic Acid.

Figure 3-18 FTIR Analysis of Chrome Ring (a) and Cylinder Liner (b) Run with Erucylamide

Figure 3-19 FTIR Analysis of Chrome Ring (a) and Cylinder Liner (b) Run with EruLin

Figure 3-20 FTIR Analysis of Cylinder Liner at the End of Stroke Position Run with EruLin.

Figure 3-21 FTIR Analysis of Molybdenum Ring (a) and Cylinder Liner (b) Run with Linoleic Acid

Figure 3-22 Optical Image (a) and FTIR Line Scan (b) of Cylinder Liner Specimen Run with Erucylamide

Figure 3-23 Wire Grid Projection of FTIR Scan on Cylinder Liner Specimen Run with Erucylamide.

Figure 3-24 Individual FTIR Spectra at 400, 4500 and 8400 microns on Cylinder Liner Specimen Run with Erucylamide

Figure 3-25 $1560 \mathrm{~cm}^{-1}$ Absorbance Variation on Cylinder Liner Specimen Run with Erucylamide

Figure 3-26 $1640 \mathrm{~cm}^{-1}$ Presence (top) and Absorbance Variation $1645 \mathrm{~cm}^{-1}$ (bottom) on Cylinder Liner Specimen Run with Erucylamide

Figure 3-27 Optical Image (a) and FTIR Line Scan of Cylinder Liner Specimen Run with Linoleic Acid

Figure 3-28 Wire Grid Projection of FTIR Scan on Cylinder Liner Specimen

Run with Linoleic Acid

Figure 3-29 Individual FTIR Spectra at 1200, 4800 and 7800 microns on

Cylinder Liner Specimen Run with Linoleic Acid. 94

Figure 3-30 Influence of Molecular Orientation on Additive Performance. 
Figure 3-31 Hypothesised Formation of Additive Films ..................................... 98

Figure 4-1 PCS Mini Traction Machine............................................................... 106

Figure 4-2 Specimen Surfaces and Analytical Techniques. After (Renondeau 2005) 108

Figure 4-3 1:1 Scale Plan View of MTM Disc Specimen Detailing Profilometer Path

Figure 4-4 Typical MTM Test Result .................................................................. 113

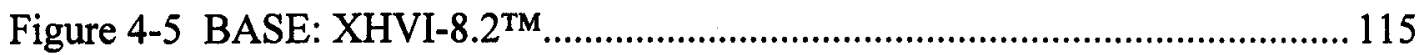

Figure 4-6 LIN: 2 wt\% Linoleic Acid in XHVI-8.2 ${ }^{\mathrm{TM}}$........................................... 116

Figure 4-7 ERU: 2 wt\% Erucylamide in XHVI-8.2 ${ }^{\mathrm{TM}}$.................................. 117

Figure 4-8 ERULIN: 1 wt\% Erucylamide + 1 wt\% Linoleic Acid in XHVI$8.2^{\mathrm{TM}}$

Figure 4-9 Averaged Friction Coefficient Data for Run 1 ( 0 mins)...................... 118

Figure 4-10 Averaged Friction Coefficient Data for Run 4 (30 mins).................. 119

Figure 4-11 BASE Post Test Disc Surface...................................................... 119

Figure 4-12 LIN Post Test Disc Surfaces - a) First Test, b) Repeat ..................... 120

Figure 4-13 ERU Post Test Disc Surfaces - a) First Test, b) Repeat.................... 120

Figure 4-14 ERULIN Post Test Disc Surfaces - a) First Test, b) Repeat ............... 121

Figure 4-15 BASE Post Test Disc Surface Profilometry Measurement................. 122

Figure 4-16 LIN Post Test Disc Surface Profilometry Measurement .................... 122

Figure 4-17 ERU Post Test Disc Surface Profilometry Measurement.................. 122

Figure 4-18 ERULIN Post Test Disc Surface Profilometry Measurement ............ 122

Figure 4-19 ERULIN Post Test Ball Surface SEM / EDX Analysis..................... 124

Figure 4-20 ERULIN Post Test Disc Surface SEM / EDX Analysis.................... 124

Figure 4-21 BASE Post Test Disc Surface XPS Analysis On Wear Track............ 126

Figure 4-22 BASE Post Test Disc Surface XPS Analysis Off Wear Track ........... 126

Figure 4-23 BASE Post Test Ball FTIR Analysis ............................................... 129

Figure 4-24 LIN Post Test FTIR Analysis on a) Ball and b) Disc Specimen ........ 129

Figure 4-25 ERU Post Test Specimen FTIR Analysis on a) Ball and b) Disc Specimen .............................................................................. 130

Figure 4-26 ERULIN Post Test Specimen FTIR Analysis.................................. 131

Figure 5-1 Engine Dynamometer Calibration .................................................. 143 
Figure 5-2 Inspection of Components After Sixth Engine Test: a) Cylinder Bore, b) Piston Crown, c) Valves, d) Piston and Connecting Rod.

Figure 5-3 Piston Assembly Friction Measurement Apparatus ............................ 146

Figure 5-4 Connecting Rod Calibration Graph ................................................. 148

Figure 5-5 Engine Torque Measurement for Test 1 ........................................... 152

Figure 5-6 Near Surface TDC Cylinder Liner Temperature for Test 1 ................. 153

Figure 5-7 Lambda Sensor Position Response for Test 1 .................................. 153

Figure 5-8 Engine Torque Measurement for Test 3 ...................................... 154

Figure 5-9 Near-surface TDC Cylinder Liner Temperature for Test 3 ................. 155

Figure 5-10 Lambda Sensor Position Response for Test 3 ................................. 155

Figure 5-11 Piston Assembly Friction Measurement and Key Engine Events:

Test 1 ................................................................................. 157

Figure 5-12 Piston Assembly Friction Around End of Stroke: Test 1 ................. 158

Figure 5-13 Piston Assembly Friction Power Loss: Test 1 ................................ 158

Figure 5-14 Piston Assembly Friction Measurement and Key Engine Events:

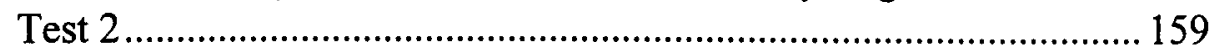

Figure 5-15 Piston Assembly Friction Around End of Stroke: Test 2 ................. 160

Figure 5-16 Piston Assembly Friction Power Loss: Test 2 .............................. 160

Figure 5-17 Piston Friction Power Loss: Test 2............................................. 161

Figure 6-1 Comparison of Results Presented by Castle and Bovington (2003) and Those Presented in Chapter 3 ................................................ 172

Figure 6-2 Comparison of Boundary Friction Coefficient for TE77 and MTM Tests

Figure 6-3 Comparison of End-of-Stroke Reversal Between a) TE-77 and b) Engine Tests

Figure 6-4 Gasoline Consumption in the UK and Calculated Saving Made Through Implementation of Friction Modified Gasoline. After (DfT 2006) 


\section{Tables}

Table 1-1 Typical Engine Lubricant Additives. After (Priest 2006)......................... 9

Table 1-2 Main Classes of Gasoline Additive in use from 1926 - 1971. After (Robinson 1977).

Table 2-1 Fuels Blends Used In Investigation .................................................. 33

Table 2-2 Top Ring Zone Sampling Eng3ine Testing Matrix ................................ 34

Table 2-3 Cold-Start Engine Test Parameters...................................................... 39

Table 2-4 Steady State Engine Test Parameters.................................................. 40

Table 2-5 Steady State Engine Test Matrix ......................................................... 40

Table 2-6 Calculated Error Associated with TPS Viscosity Measurement.............. 43

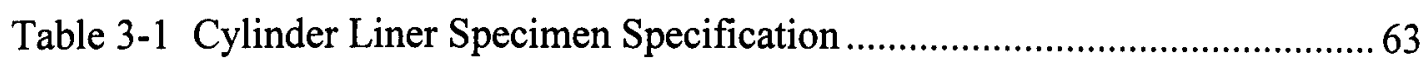

Table 3-2 Running-In Conditions for Piston Ring and Cylinder Liner

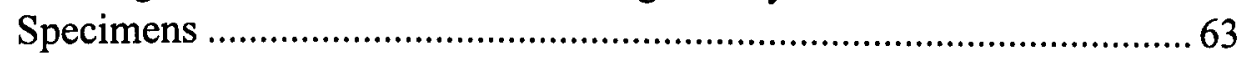

Table 3-3 Organic Friction Modifiers Under Investigation .....................................6 65

Table 3-4 Additive Screening Test Conditions ..................................................6 67

Table 3-5 Coefficient of Friction Comparison .................................................... 83

Table 4-1 Specification of Ricardo Hydra Cylinder Liner and Bespoke MTM Disc Specimen

Table 4-2 Portfolio of Surface Analysis Techniques Employed on MTM Specimens

Table 4-3 Test Lubricant Formulations and Dynamic Viscosity at $100^{\circ} \mathrm{C}$ 114

Table 4-4 Wear Area Measurements

Table 4-5 Average Atomic \% Over Information Depth

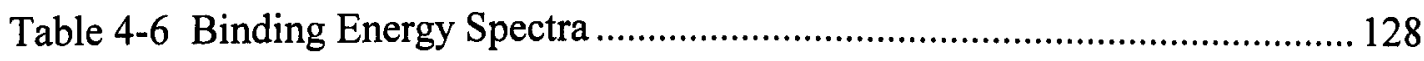

Table 5-1 Fuels Used in Engine Tests........................................................... 141

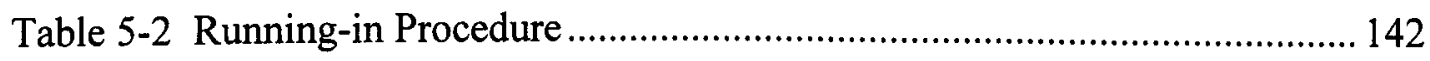

Table 5-3 Engine Performance Test Conditions ............................................... 143

Table 5-4 Engine Flushing Procedure ............................................................ 144

Table 5-5 Standard Engine Friction Test Conditions........................................ 148

Table 5-6 Friction Data Capture Procedure ...................................................... 149

Table 5-7 Sump Lubricant Sample Fuel and Linoleic Acid Concentration ........... 162 
Table 6-1 Comparison of Sump Fuel Dilution Findings....................................... 170

Table 6-2 Comparison of Test Conditions for Additive Screening Tests............... 172

Table 6-3 Comparison of Key Test Parameters in TE-77 and MTM Tests ........... 174 


\section{Abbreviations}

AAAE

ABDC

AFR

AISI

ASTM

ATDC

$\mathrm{BBC}$

BBDC

$\mathrm{BC}$

BDC

BHN

BP

BTDC

CAD

CHON

DfT

EDX

FM

FTIR

GC

GM

GNP

HB

HBP

HFRR

ICP-AES
Acetic Acid Alkyl Esters

After Bottom Dead Centre

Air / Fuel Ratio

American Iron and Steel Institute

American Society for Testing and Materials

After Top Dead Centre

British Broadcasting Corporation

Before Bottom Dead Centre

Before Christ

Bottom Dead Centre

Brinell Hardness Number

British Petroleum

Before Top Dead Centre

Computer Aided Design

Carbon, Hydrogen, Oxygen and/or Nitrogen

Department for Transport

Energy Dispersive X-Ray (Spectroscopy)

Friction Modifier

Fourier Transform Infrared (Spectroscopy)

Gas Chromatography

General Motors

Gross National Product

Brinell Hardness

High Boiling Point

High Frequency Reciprocator Rig

Inductively Coupled Plasma Atomic Emission Spectrophotometry 
ICP-MS Inductively Coupled Plasma Mass Spectrometry

ILSAC International Lubricants Standardisation Committee

IMEP Instantaneous Mean Effective Pressure

IR Infrared

MoDTC Molybdenum Dithiocarbamate

MP Melting Point

MTM Mini Traction Machine

NOCH Nitrogen, Oxygen, Carbon, Hydrogen

OEM Original Equipment Manufacturer

PC Personal Computer

PCI Peripheral Component Interconnect

PTFE Polytetraflouroethylene

RMS Root Mean Square

RPM Revolutions Per Minute

RON Research Octane Number

SAE Society of Automotive Engineers

SBP Special Boiling Point

SEM Scanning Electron Microscopy

SRR Slide / Roll Ratio

TDC Top Dead Centre

TPS Three Point Sampling

TRZ Top Ring Zone

UK United Kingdom

ULG Unleaded Gasoline

UNECE United Nations Economic Commission for Europe

XHVI Extra High Viscosity Index

XPS X-Ray Photoelectron Spectroscopy 


\section{Notation}

$\begin{array}{cl}\mathrm{A}_{\mathrm{p}} & \text { Cross Section Area of Piston } \\ \mathrm{a}_{0} & \text { Piston Assembly Acceleration } \\ f & \text { Friction Force } \\ \mathrm{F}_{\mathrm{STG}} & \text { Forces Measured by Connecting Rod Strain Gauge } \\ \mathrm{F}_{\mathrm{c}} & \text { Forces Acting Along the Connecting Rod Axis } \\ \mathrm{g} & \text { Gravity } \\ \mathrm{m}_{\mathrm{p}} & \text { Piston Assembly Mass } \\ \eta_{f} & \text { Dynamic Viscosity of Fuel } \\ \eta_{o} & \text { Dynamic Viscosity of Oil } \\ \eta_{m} & \text { Dynamic Viscosity of Mixture } \\ \mathrm{P}_{1} & \text { Cylinder Pressure } \\ \mathrm{P}_{2} & \text { Crankcase Pressure } \\ x_{f} & \text { Proportion of Fuel in Mixture } \\ x_{o} & \text { Proportion of Oil in Mixture } \\ \mathrm{U}_{1} & \text { Velocity of Specimen 1 } \\ \mathrm{U}_{2} & \text { Velocity of Specimen } 2 \\ \lambda & \text { Equivalence Ratio } \\ \phi & \text { Crankshaft Attitude Angle } \\ \end{array}$




\section{Introduction}

The demand for oil products continues to rise on a global scale. The world wide dependence on oil causes the consumption of over 82 million barrels per day, which equates to 13,036 million litres combusted each day (B.P. 2005). Given the quantity of the planet's remaining proved reserves, at this rate of consumption there is only enough oil to sustain 40 years of oil fuelled activity. The reciprocating internal combustion engine in its many forms contributes a significant proportion to the consumption of oil resources and is said by some to be crucial to the economic success of all nations of the world and to the quality of life of their citizens (Priest 1996). In the UK, our modern lifestyles ensure we encounter a vast array of internal combustion engine powered systems on a daily basis. Cars, motorcycles, taxis, vans, buses, coaches, trains, planes, tractors and boats, to mention but a few, are the hub of modern living, all relying on the reciprocating internal combustion engine whose efficient and reliable nature makes it the unsung hero of the $21^{\text {st }}$ century.

The road transport industry consumes a vast amount of oil products in comparison to its counterpart transport systems of railway, water and aviation. This is primarily due to the success of the 'Private and Light Goods', or 'Private Motor Vehicle', whose numbers rose to approximately 30 million in the UK in 2005 (DfT 2006). The number of households in possession of a motor vehicle also rises every year. In 2005 it is reported that $75 \%$ of the population of the British Isles owned a private motor vehicle, with $32 \%$ of that amount owning two or more. Although the public's appetite for gasoline powered vehicles has decreased over recent years in preference of the more efficient diesel powered vehicle, gasoline powered vehicles still represents $72 \%$ of the market.

Despite the evolution of what is now a deeply embedded culture of dependence on oil products, inhabitants of the world are more environmentally aware than ever before. An increased awareness of the link between greenhouse gas emissions and global warming means that there is a desire to reduce carbon dioxide emissions from transportation. Carbon dioxide is the most important greenhouse gas and is estimated to account for about two thirds of man made global warming. Although carbon dioxide is less influential on global warming than many other greenhouse gases, it is present in our atmosphere in vastly greater quantities. Domestic transport systems alone contribute over 43 million tonnes of carbon as a result of carbon dioxide emissions in the British isles each year (DfT 2006). However, the burning of hydrocarbon fuels causes the emission of several other gases whose release is tightly regulated for the reasons given below (DfT 2006). 
- Carbon monoxide is emitted as a result of the incomplete combustion of hydrocarbon fuels and is thought to be one of the most directly toxic substances, interfering with the respiratory system bio-chemistry of the body and affecting the central nervous and cardiovascular systems.

- Nitrogen oxides are also formed in the combustion of carbon fuels, like carbon monoxide they harm the respiratory system and reduce lung function. Their presence also contributes to the formation of ozone, a secondary pollutant which contributes to global warming.

- Benzene, a well known human carcinogen, and butadiene, a suspected human carcinogen are also emitted when burning carbon fuels.

- Sulphur dioxide, an acid gas, is also emitted in the burning of hydrocarbon fuels and can affect both health and vegetation. Its damaging effects on the environment have caused it to be recognised by the United Nations Economic Commission for Europe (UNECE), leading to stringent legislation detailing acceptable emission levels.

The general public's knowledge of the problems associated with the global dependence on hydrocarbon fuels cause governments to respond with legislation to control the situation, Figure 1-1.

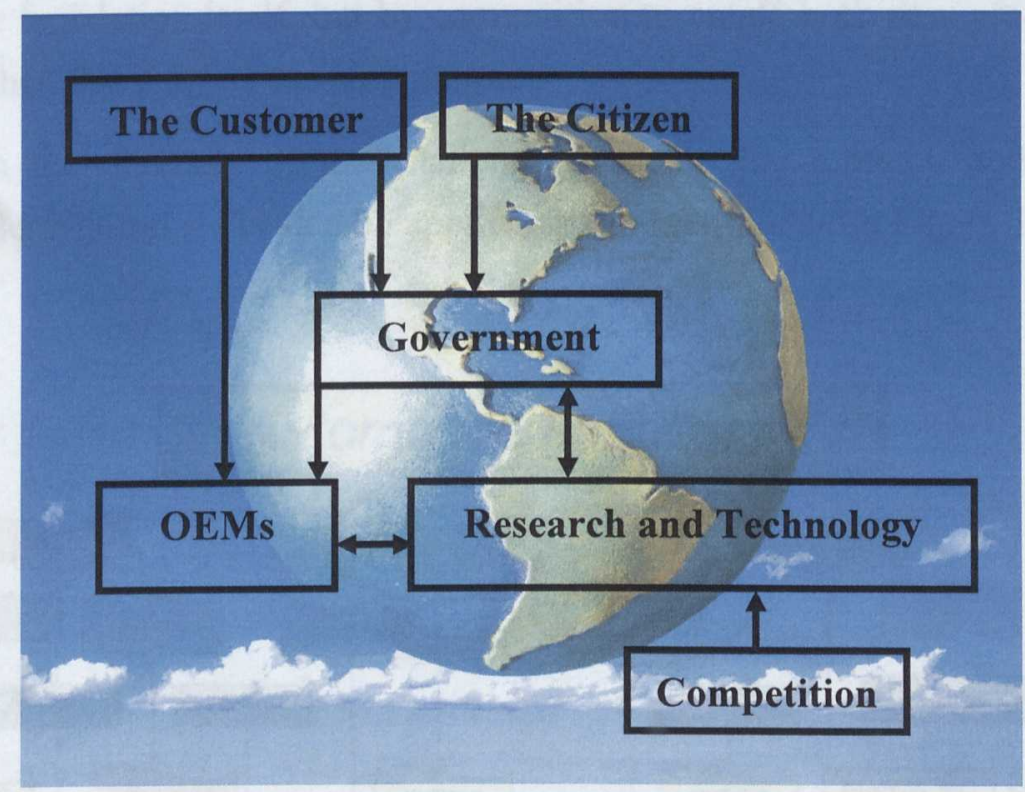

Figure 1-1 Global Information Flows Governing Legislation

The customer, citizen or society puts pressure on the government to legislate the use of products which have the potential to significantly impact on the environment. The motor car is one of these products. As a result the Original 
Equipment Manufacturers (OEMs) create a specification of vehicle which fits the requirements. From this point it is the task of researchers and the technology industries to create products in keeping with the needs of society. However, close inspection of Figure 1-1 reveals there are reciprocal lines of communication between the researchers and both the government and OEMs. The research conducted by the research industry can often feedback directly to the UK government influencing the creation of specific legislation. A report generated for the government (Jost 1966) reported that simply through the effective application of existing knowledge and techniques in tribology, UK industry could make annual savings in operating costs of $£ 515$ million. According to some researchers/analysts, the direct cost of friction and wear can account for nearly $10 \%$ of the gross national product (GNP) in many industrial nations. In the case of lubricants, it is suggested that the correct selection of a lubricant for a job could save up to $1 \%$ of the GNP (Taylor 2002).

As discussed above, the internal combustion engine takes many forms and the diversity of its application is truly vast; thus the requirements of each engine's fluids differs immensely. To ensure efficient, reliable operation bespoke products must be created for each application, based on a deep knowledge and understanding of the environment in which the machine operates. This understanding further complicates the task of the research and technology industries that respond to, and inform the creation of, legislation. As described in the above diagram, this industry is fuelled both by the legislation itself, but by competition to create both new products and to maximise the efficiency of existing products.

Given the diversity of the challenge posed to the research and technology industries, legislation is divided by application and requirement, Figure 1-2.

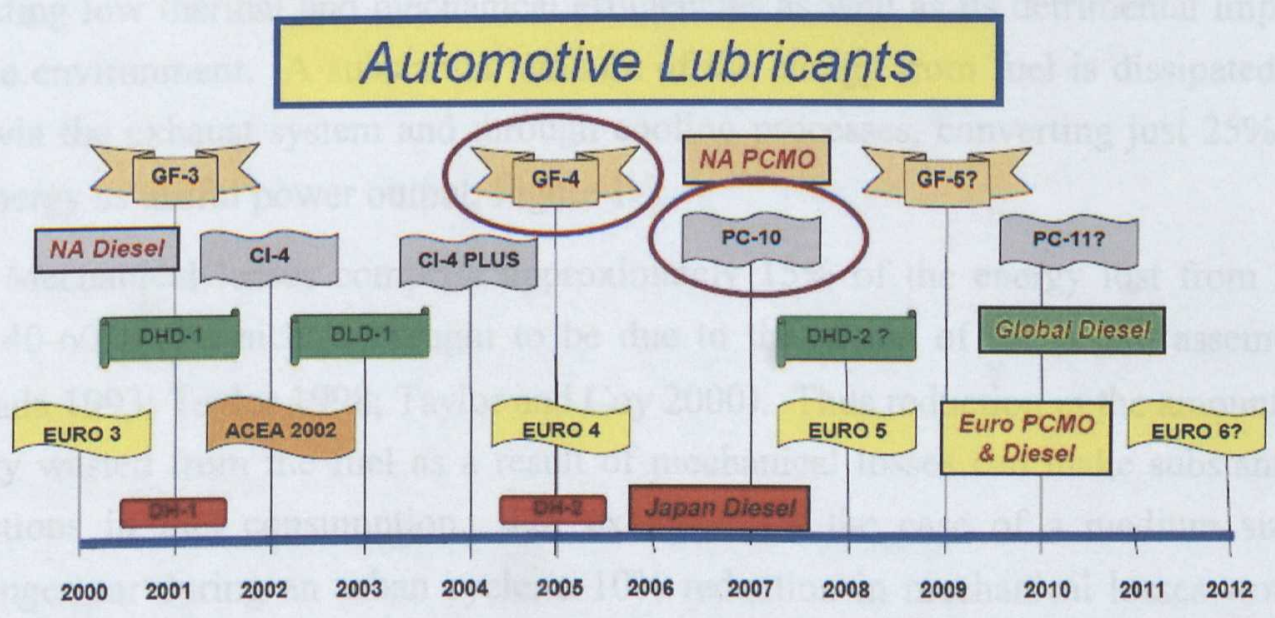

Figure 1-2 Automotive Lubricant Legislation (Bardasz 2005) 
The two key types of legislation which dictate the lubricant performance requirements of both gasoline and diesel engines are the GF and PC standards. The GF type legislation dictates the minimum performance requirements (both engine and bench tests) and chemical and physical properties for those engine oils that vehicle manufacturers deem necessary for satisfactory equipment performance and life (ILSAC 2004). Discussions detailing the exact specification of GF-5 lubricants are ongoing, although it is thought they will promote fuel economy to reduce both hydrocarbon emissions and consumption. The PC type legislation dictates the minimum performance of lubricant used in diesel engines equipped with exhaust after treatment and run on ultra-low sulphur fuel. Thus the current PC-10 lubricant specification evolved from a need to reduce harmful emissions, and further fuel economy.

This thesis addresses the issue of furthering fuel economy both to reduce hydrocarbon consumption and vehicle emissions. With a global understanding of the problem and an appreciation of the current technologies and research, this thesis applies knowledge, systematic research, logic, innovation and creativity in pursuit of this goal. Research conducted in this project is founded on the belief that successfully achieving the ever more challenging goal of increasing fuel economy lies in bridging the gap between chemistry, material science, surface science, engineering and tribology. To this end, this opening chapter serves to present the diverse problems associated with the reciprocating internal combustion engine, as well as to review other research work in the literature on furthering fuel economy.

\subsection{Engine Friction and Lubrication}

Despite its popularity, the internal combustion engine has many drawbacks including low thermal and mechanical efficiencies as well as its detrimental impact on the environment. A substantial amount of the energy from fuel is dissipated as heat via the exhaust system and through cooling processes, converting just $25 \%$ of the energy as useful power output, Figure 1-3.

Mechanical losses comprise approximately $15 \%$ of the energy lost from the fuel, $40-60 \%$ of which is thought to be due to the action of the piston assembly (Nakada 1993; Taylor 1998; Taylor and Coy 2000). Thus reduction in the amount of energy wasted from the fuel as a result of mechanical losses can make substantial reductions in fuel consumption. For example, for the case of a medium sized passenger car during an urban cycle, a $10 \%$ reduction in mechanical losses would lead to a $1.5 \%$ reduction in fuel consumption (Priest 2000). An understanding of the role of the piston assembly and the environment in which it operates is therefore 
essential when identifying the system as one for friction reduction as a result of improved tribology.

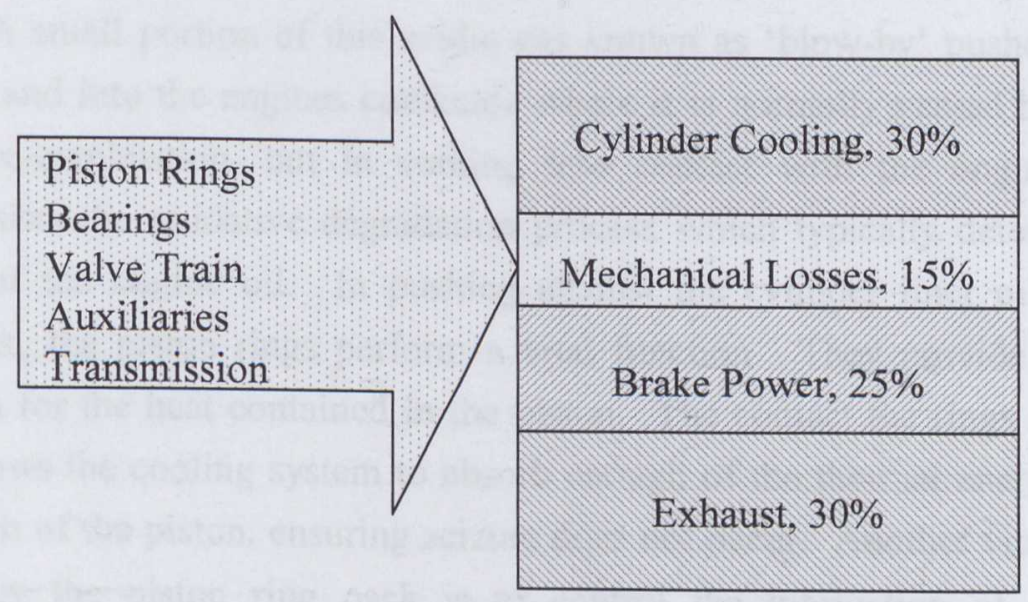

Figure 1-3 Typical Fuel Energy Distribution in an Internal Combustion Engine. After (Taylor 1998).

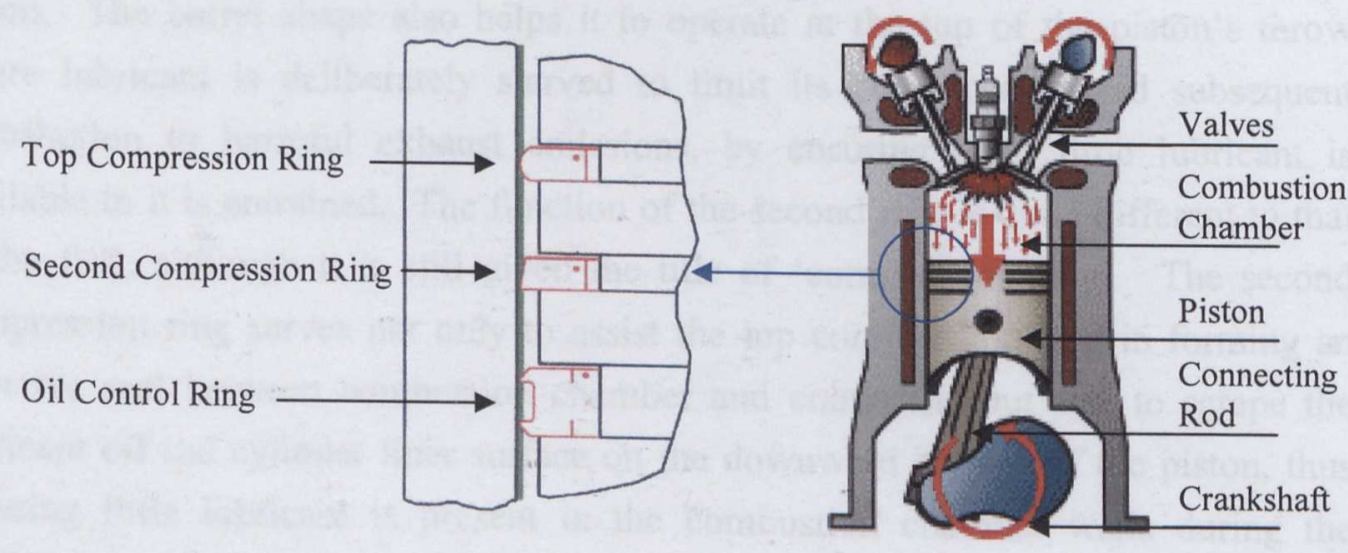

Figure 1-4 Typical Four-Stroke Gasoline Engine Cylinder Layout and Piston Assembly. After (Priest 1996; Subaru 2006).

The piston assembly is a system of components whose primary function is to efficiently transfer the energy from the combustion of hydrocarbon fuel into useful work, Figure 1-4. As a result the piston assembly operates in one of the most arduous environments found in any machine. It is subjected to high combustion temperatures and pressures which are repeated thousands of times a minute presenting a need for it to resist thermal shock and cyclic fatigue. The products of the combustion process are often acidic and come into direct contact with the assembly as a result of the extremely high pressure gas which forces the piston's 
motion. This high pressure gas acts behind the piston rings forcing them to push against the cylinder liner which constrains their radial expansion. Here a thin film of lubricant exists to reduce the friction between the piston ring and cylinder liner, reducing wear and thereby ensuring the smooth running and satisfactory life of the assembly. A small portion of this acidic gas known as 'blow-by' pushes past the piston rings and into the engines crankcase where it is normally vented back to the intake for re-combustion, but in coming into contact with the engine's sump lubricant assists the oxidative degradation process which typically determines the useful life of the engine oil. In pushing against the cylinder liner oil film and cylinder liner, the piston rings perform a vital function. They provide a thermal transfer path for the heat contained in the piston. The contact the rings make with the bore allows the cooling system to absorb enough of the thermal energy to limit the expansion of the piston, ensuring seizure does not occur. Another vital function performed by the piston ring pack is to control the lubrication of the piston assembly. As shown in Figure 1-4 each ring in the ring pack is specially designed to perform an individual role. The first ring is typically barrel shaped and is known as the 'top compression ring'. As discussed above, its function is to effectively seal the combustion chamber from the crankcase, and to provide a cooling path for the piston. The barrel shape also helps it to operate at the top of the piston's throw where lubricant is deliberately starved to limit its consumption and subsequent contribution to harmful exhaust emissions, by ensuring what little lubricant is available to it is entrained. The function of the second ring is quite different to that of the first, although it is still given the title of 'compression ring'. The second compression ring serves not only to assist the top compression ring in forming an effective seal between combustion chamber and crankcase, but acts to scrape the lubricant off the cylinder liner surface on the downward strokes of the piston, thus ensuring little lubricant is present in the combustion chamber walls during the combustion event and limiting the consumption of lubricant during the process. Due to the action of the second ring, it is often called the 'scraper ring'. However, the tapered face of the ring allows oil to be transported up the cylinder liner on the upstroke of the piston; its wedged shape profile promotes fluid lubrication. The third piston ring is termed the 'oil control ring'. As the name suggests it is designed to control the flow of oil to the piston assembly, ensuring there is adequate oil for the systems operation, but not allowing excessive oil transport which might result in large amounts of lubricant entering the combustion chamber and being consumed.

Unlike any other significant component of the internal combustion engine, primarily due to the reciprocating action, the lubrication of the piston assembly spans all major lubrication regimes, Figure 1-5. 
When the piston assembly is at both the top and bottom of the cylinder, at the ends of its stroke, the velocity of the piston is low enough to allow the assembly to enter the boundary lubrication regime. In this regime the surfaces are in normal contact and thus the bulk properties of the lubricant are no longer of importance, and lubrication relies on chemical and physical action of thin molecular films. At a certain point in the stroke of the piston, shortly after top of bottom dead centre, velocity increases enough to allow the entrainment of some lubricant. At this point there is both asperity interaction and some fluid lubrication, thus this type of lubrication is known as 'mixed'. With velocity increased further, the piston enters the elastohydrodynamic regime. In this regime fluid film lubrication dominates and surfaces are separated, but a more concentrated mechanism where elastic deformation of the surfaces occurs as a result of the effect of pressure on viscosity. Around the mid-stroke of the piston, the velocities are high enough to ensure full fluid film lubrication in which the surfaces are completely separated and the dynamic viscosity of the fluid becomes the most important property. Traditionally it is in this regime that the piston assembly spends most of its time. It is therefore the energy required to shear the fluid under hydrodynamic conditions that dominates the level of resistance to motion, and consequently the friction of the piston assembly's motion, during this regime.

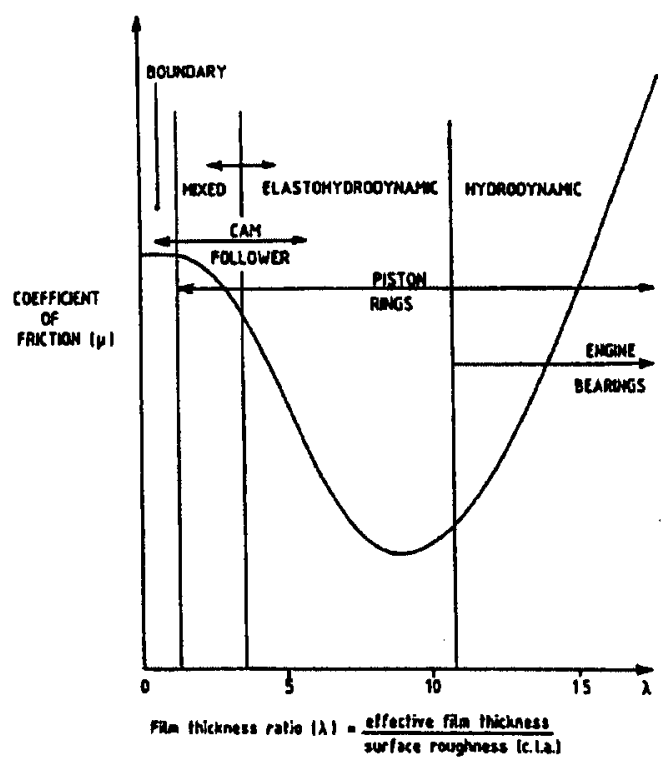

Figure 1-5 Modified Stribeck Diagram (Priest 2000)

\subsection{Engine Lubricants and Formulation}

The main role of the lubricant is to minimise friction and wear in the contact between piston rings and cylinder liner. However, increasingly stringent legislation 
is calling for lubricants to overcome a series of conflicting requirements. Lubricants should increase fuel economy, reduce emissions, increase engine durability and have extended drain intervals, perhaps even stay in service for the life of the vehicle. They key trends over the past few years have primarily been driven by the need for improved fuel economy, although the need for improved oxidation stability, handling of contaminants and compatibility with other systems has played a part. To date, increases in fuel economy have primarily been achieved through the lowering of the bulk viscosity of the engine oil. In most typical engines the dominating lubrication regime during piston assembly operation is hydrodynamic, thus the lower viscosity fluid requires less energy to shear it reducing the contribution of this lubrication regime to the total friction. However, as the viscosity is reduced, the point in the cycle where hydrodynamic passes into the elastohydrodynamic, elastohydrodynamic into mixed, and mixed into the boundary lubrication regime occurs earlier allowing these regimes to have a more dominant effect on the overall friction of the system (Taylor, Brown et al. 1994). Essentially, where low viscosity lubricant is used, the piston assembly spends a greater amount of time in lubrication regimes where metal-to-metal contact occurs having serious implications for friction and wear. Once in these contact regimes the bulk properties of the lubricant become less important, and it is the task of the lubricant additive package to control friction and wear thus making the choice of a lower viscosity lubricant valid.

Approximately $5 \%-25 \%$ of a typical engine lubricant is made up of additives (Taylor 2002; Moreton 2005). Additives are chemicals added to a lubricant to perform specific functions or achieve levels of performance not possible with the base oil alone. The basic types of lubricant additive are presented in Table 1-1.

To permit the use of a lubricant with low viscosity, a lubricant must have an additive package strong in anti-wear, extreme pressure and friction modifier additives. From inspection of the above table it is clear that the preferred anti-wear and extreme pressure additives are zinc dialklydithophosphates (ZDDPs). ZDDPs are arguably the most successful lubricant additives ever invented. They are still in use today, and have been for approximately 60 years, despite attempts from additive companies to replace them. Their remarkable anti-wear, antioxidant and corrosion inhibiting properties coupled with their cost-effectiveness continues to ensure their place in the automotive additive market. This triple purpose additive has inspired vast amounts of research into its action since the day it was first introduced, making a review of the history, or mechanisms of action, of ZDDPs impractical in this thesis. However, a number of good reviews discussing both the history and the current state of knowledge on ZDDPs have been written and it is recommended that the interested reader refers to these for in-depth information on the subject (Tripaldi, 
Vettor et al. 1996; Spikes 2004; Nicholls, Do et al. 2005). However, it is worth briefly discussing the main mechanism by which ZDDPs work. The interposition of a surface film prevents metal surfaces from contacting each other during mild conditions. Under more severe conditions they act as extreme pressure additives forming metal sulphides and a phosphate glass protecting surfaces from metal-tometal contact. Despite the vast amount of knowledge gained on ZDDPs as a result of the last 60 years of research there are still several important gaps in our understanding of exactly how they work, although much is known about their effects, both beneficial and detrimental. It is reported that in some situations ZDDPs can produce significantly higher friction contacts, particularly at higher operating temperatures and in the mixed lubrication regime, than additive-free base oil (Tripaldi, Vettor et al. 1996).

\begin{tabular}{|c|c|}
\hline Additive Type & Description \\
\hline Acid Neutraliser & $\begin{array}{l}\text { Neutralise acidity resulting from oxidation and } \\
\text { contamination }\end{array}$ \\
\hline Anti-foam & $\begin{array}{l}\text { Reduce the tendency of the lubricant to foam, or } \\
\text { to reduce the life of the foam. }\end{array}$ \\
\hline Antioxidant & $\begin{array}{l}\text { Reduce or delay oxidation which leads to } \\
\text { lubricant degradation. }\end{array}$ \\
\hline Anti-wear & $\begin{array}{c}\text { Reduce wear and prevent scuffing of rubbed } \\
\text { surfaces. Zinc Dialkyldithiophosphate (ZDDP) } \\
\text { most common. }\end{array}$ \\
\hline Corrosion Inhibitors & $\begin{array}{l}\text { Reduce corrosion by the formation of a } \\
\text { protective surface film. }\end{array}$ \\
\hline Detergents & $\begin{array}{l}\text { Reduce or prevent insoluble deposits formed on } \\
\text { surfaces at high temperatures. }\end{array}$ \\
\hline Dispersants & $\begin{array}{l}\text { Prevent deposition by dispersing finely divided } \\
\text { suspension of insoluble material formed at low } \\
\text { temperature }\end{array}$ \\
\hline Emulsifiers & $\begin{array}{c}\text { Form either water-in-oil or oil-in-water } \\
\text { emulsions. }\end{array}$ \\
\hline Extreme Pressure & $\begin{array}{l}\text { Prevent scuffing of rubbed surfaces under shock } \\
\text { and very high loads. Zinc Dialklydithiophosphate } \\
\text { (ZDDP) most popular. }\end{array}$ \\
\hline Friction Modifiers & $\begin{array}{l}\text { Reduce friction when surfaces come into contact } \\
\text { by the formation of surface films. Molybdenum } \\
\text { Dithiocarbamate (MoDTC) most popular. }\end{array}$ \\
\hline Pour Point Depressant & $\begin{array}{l}\text { Reduce the pour point of oils containing } \\
\text { paraffinic compounds }\end{array}$ \\
\hline Tackiness & $\begin{array}{l}\text { Reduce the loss of oil from surfaces by gravity or } \\
\text { centrifugal effects. }\end{array}$ \\
\hline Viscosity Index Improvers & $\begin{array}{l}\text { Reduce the decrease in viscosity due to the } \\
\text { increase in temperature }\end{array}$ \\
\hline
\end{tabular}

Table 1-1 Typical Engine Lubricant Additives. After (Priest 2006). 
The key factor which brings about the demise of ZDDP from the formulated lubricant is the fact that phosphorus, a core component of the additive, causes exhaust catalyst poisoning (Korcek and Nakada 1996). The catalyst, or catalytic converter, is located in the exhaust system of the motor vehicle, and is responsible for treating the exhaust before it leaves the vehicle, removing many of the harmful pollutants. A typical catalyst consists of a ceramic structure with a large surface area coated with a metal catalyst, usually platinum, rhodium and/or palladium. Platinum and rhodium help to reduce the NOx emissions by taking the nitrogen atom out of the molecule and releasing $\mathrm{O}_{2}$. The palladium acts as an oxidation catalyst reducing unburned hydrocarbons and carbon monoxide molecules to less harmful compounds (Nice 2007). Phosphorus from the engine oil can contribute to the irreversible deactivation of the catalyst resulting in increased emissions (Bardasz 2005). It is also known that sulphated ash from the engine oil can contribute to ash blockage in catalysts resulting in raised back pressure on the engine and a fuel economy penalty, Figure 1-6.

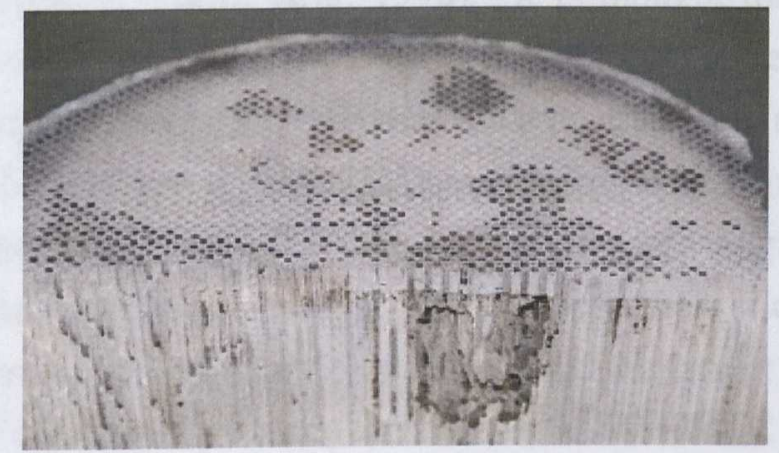

Section of a Catalyst

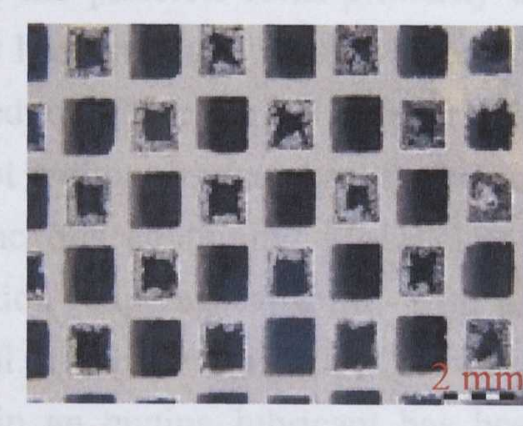

Ash Blockage in Catalyst

Figure 1-6 Sulphated Ash Blockage in Catalyst (Bardasz 2005)

Friction modifiers are also a key component of the additive package, especially when low viscosity lubricants are used. As presented in Table 1-1, the most common friction modifier lubricant additive is molybdenum dithiocarbamate (MoDTC). Like ZDDP, this additive has been in service for many years. Although not considering the additive as a means of energy conservation, a critical analysis of the performance of molybdenum compounds in motor vehicles in 1978 suggested that successful application of the additive could reap a 3\% improvement in mechanical efficiency, together with a $4 \%$ improvement in final drive efficiency (Braithwaite and Greene 1978). The research explains that the technology of stable dispersions of solid lubricants, including $\mathrm{MoS}_{2}$, was at an advanced stage by the 1960 's although the motorist found it difficult to quantitatively assess the $3 \%$ saving 
in fuel consumption resulting from the addition of $\mathrm{MoS}_{2}$ to crankcase oil. It was the 'energy crisis' situation of the late 1970s that began to force research into the area, as vehicle manufacturers became interested in fuel economy as a differentiator in the automotive market place. By 1984 the use of Mo-S compounds was reported to be 'well established' as anti-wear and extreme pressure additives in lubricating oils and greases where they reduced friction and wear of sliding surfaces (Mitchell 1984). The molybdenum dithiophosphates action is not dissimilar to that of the zinc dithiophosphates, where their tribological action results from their decomposition in the bulk oil; the successful lubricating action comes from the sulphur containing decomposition products. In that respect, the metal complexes (ZDDP and MoDTC) can be thought of as the precursors of the tribologically active substances.

Essentially the main way that solutions of organo-molybdenum additives such as MoDTCs reduce friction is by interposing a thin film of $\mathrm{MoS}_{2}$, which has an inherent low shear strength, into the rubbing contact (Graham, Spikes et al. 2001). The generation of this $\mathrm{MoS}_{2}$ film, made up of tiny platelets, appears to require rubbing and thus takes place only in the mixed and boundary lubrication regimes (Grossiard, Varlot et al. 1998). It is thought that the platelets form primarily on asperity peaks and thus support most of the applied load in the boundary lubrication regime, consequently having a large effect on reducing friction. However, like ZDDP, the use of MoDTC in engine lubricant is not without its drawbacks. Several authors have reported that fully formulated engine oils containing MoDTC as a friction reducing additive gradually lose their friction reducing capability with use (Korcek, Jensen et al. 2000; Yamada, Ishimaru et al. 2000; Jensen 2005). However the combined presence of ZDDPs and MoDTC in an engine lubricant has been shown to be beneficial as zinc containing products were found to be stronger antioxidants than the corresponding molybdenum compound. In lubricants where ZDDPs had been consumed the complete loss of friction reducing capability was found to occur, probably due to the polar base oil oxidation products and components which interfere with the action of the MoDTC. So for a truly effective formulation large amounts of both ZDDPs and MoDTC are required to give the lubricant good antioxidant, anti-wear and low friction properties. As discussed above, the use of large amounts such chemicals has a detrimental effect on catalysts and after treatment systems resulting in increased harmful emissions. As a result the use of such chemicals is gradually being phased out.

\subsection{Lubricant Formulations for Fuel Economy}

The main trend in engine lubricants over the last $10-15$ years have required formulations to have better antioxidant performance, giving them longer service 
lives, better dispersant - detergent performance to help maintain cleanliness and efficiency of engines, have better compatibility with after treatment devices and to give a vehicle improved fuel economy performance (Taylor, Mainwaring et al. 2005). There is a direct correlation between furthering fuel economy and reducing hydrocarbon consumption and the emission of $\mathrm{CO}_{2}$. For this reason the literature is heavily populated by OEMs reporting the energy savings they have made through successfully employing low viscosity lubricants with heavy additive packages, albeit with some interesting side effects. In the early 1990's, for example, a study reported significant energy savings by employing low viscosity engine oils in urban running conditions, resulting in limited fuel consumption and reduced $\mathrm{CO}_{2}$ emissions (Manni, Gommellini et al. 1995). However, the paper also reports an important consequence of running low viscosity oil in a vehicle equipped with standard electronic engine configuration unit; an increase hydrocarbon and carbon monoxide emissions, primarily due to the inherent increased volatility of low viscosity hydrocarbon base stocks.

Studies conducted in the late 1990's, when fuel economy was becoming a significant issue to the automotive industry, report significant fuel economy improvements through the application of low viscosity lubricants equipped with MoDTC friction modifiers, but also begin to investigate other organic friction modifier species (Hoshino, Kawai et al. 1998; Tseregounis, Mcmillan et al. 1998). No real difference was seen in the performance of the two types of friction modifier (organic and Mo), which were both tested to perform equally well, again excelling in the urban driving cycle tests. In the early 2000's, work conducted by the same authors shows the experimental technique was accurate enough to begin to differentiate between organic and Mo based friction modifiers, and research suggests that the Mo-type friction modifiers were about $0.5 \%$ more effective than their purely organic counterparts (Tseregounis and McMillan 2001).

A set of experiments conducted in 2004 tested an SAE 5W30 and a SAE 0W20 without friction modifier against each other (Mufti 2004). Tests were conducted on a research engine equipped with Instantaneous Mean Effective Pressure (IMEP) equipment, presented later in this thesis, which permits the direct measurement of piston assembly friction during firing engine conditions. The measured data showed the benefit of a high viscosity lubricant on piston assembly friction at high temperatures, and the opposite effect at low temperatures. This piece of research stresses the need for friction modifiers where low viscosity base oils are employed, particularly at high temperatures or in engines with high power densities, where lubrication conditions are significantly influenced by metal-to-metal contact. 
There are many ways in which fuel efficiency and emission control of the internal combustion engine can be achieved. Lubricant technology has been, and is, one of the most powerful tools currently available and is continually used to meet conflicting requirements dictated by engine technology, emission control and the customer. One of these conflicting requirements is to further fuel economy, but to phase out the two most powerful and efficient additives which are currently responsible for the fuel economy gains we have seen in the past $10-15$ years, ZDDP and MoDTC. It has been said by leading researchers that some of the requirements challenging the automotive industry are often demanding, contradictory, and that others cannot be met without a "breakthrough" (Korcek, Sorab et al. 2000). The authors stress that there were many tribological issues to be solved to successfully meet future emission and fuel economy requirements and that there was an urgent need for extensive systematic lubrication research to meet future technological challenges and competitive pressures. The need for alternative friction modifiers whose action does not affect other important vehicle systems manifests itself as one of these challenges. In this chapter, indeed this thesis, systematic, scientifically guided, creative and innovative research into viable friction modifier alternatives to replace MoDTC is presented.

\subsection{Friction Modifiers}

The success of MoDTC, or $\mathrm{MoS}_{2}$, has overshadowed the knowledge of any other friction modifier for many years, and has been the focus of most recently published literature. However, friction modifiers or 'boundary lubricants' have been the subject of tribological study for many years. Work conducted in the 1940's by Bowden and Tabor (1950) presents an extensive and systematic approach to the investigation into boundary lubricants. It was recognised that boundary lubrication was of great importance in engineering practice, as it governed the behaviour of most sliding mechanisms and determined whether serious wear or seizure would take place. Early experiments were conducted with a homologous series of paraffins, alcohols and fatty acids. It was shown that a small trace of a fatty acid to a mineral oil could bring about a considerable reduction in the friction and wear of a contact.

The term 'fatty acids', as used in the context of this thesis, relates to acids which have their origins in land and marine animal fats, vegetable seed oils and organic synthesis (Pryde 1985). The term was originally applied to the saturated monobasic carboxylic acids and long-chain acids which were scientifically identified as early as 1818 , although evidence suggests that they were used by the early Egyptians as early as 3200 B.C. as food stuffs. It is not thought that they were used 
for lubrication until 1400 B.C. where they were employed to lubricate Egyptian chariots. They have been known to be used in a variety of applications including arts, technology and medicine, although they are most well known for their use in soaps. Fatty acid based soaps were made by the Phoenicians as early as 600 B.C. and the ingredient remains key in the manufacture of soaps today, albeit in a more refined condition (Markley 1960). The use of fatty acids as friction modifiers in today's lubricant formulations seems to be unlikely, although it is essentially the same properties which make them a key ingredient in soaps that makes them perform well as friction modifiers. The molecules feature reactive polar heads and long hydrocarbon tails which makes them ideal for application in engine lubricant. Their long hydrocarbon tails make them immediately miscible with hydrocarbon base oils, and their reactive heads attraction to metal surfaces ensures they form on metal surfaces. The strong lateral adhesion between the molecules themselves ensures ordered and densely packed films capable of separating surfaces as described in Figure 1-7.

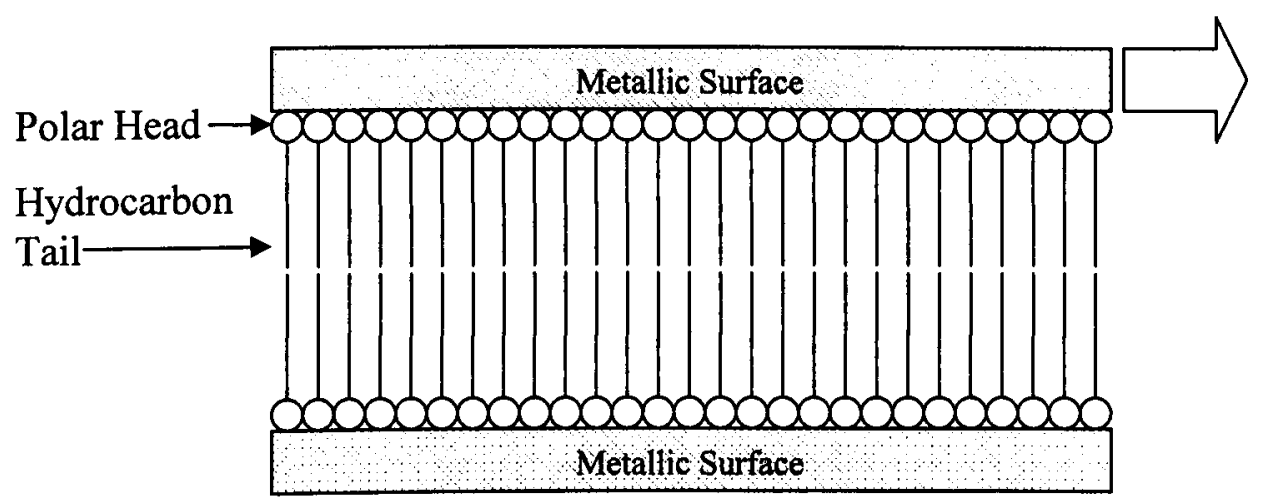

Figure 1-7 Fatty Acid Formation on Ideal Surfaces

Further experiments conducted by Bowden and Tabor revealed that effective friction reduction could be achieved with a boundary film of fatty acid just 1 or 2 molecules thick. However, tests showed that the boundary lubricating properties of fatty acids were profoundly affected by the nature of the metal surfaces. The metals which are most readily attacked by the fatty acid molecules were shown to be those which were most effectively lubricated. Effective lubrication for the less reactive metals such as aluminium and iron, which were not well lubricated by trace amounts of fatty acid, could be achieved by using stronger concentrations. The literature states 'lubrication is effected not by the fatty acid itself but by the metallic soap formed as a result of chemical reaction between the metal and the fatty acid'. It was also discussed that the breakdown of effective lubrication does not occur at the 
temperature at which the fatty acid melts but at considerably higher temperatures, where it is thought the metallic soap melts, Figure 1-8.

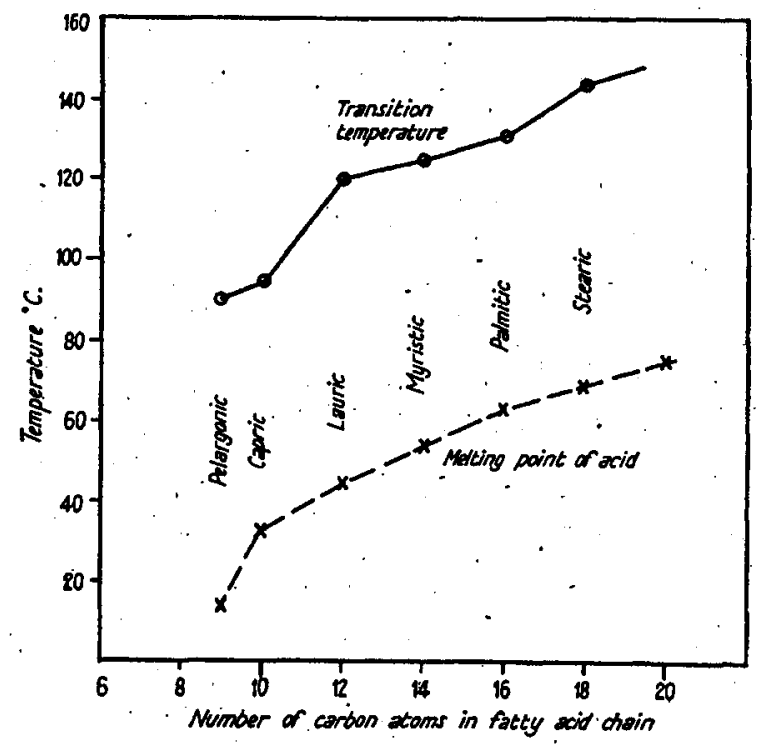

Figure 1-8 Breakdown or Transition Temperature of Fatty Acids on Steel Surfaces

(Bowden and Tabor 1950)

It is also apparent on inspection of the above figure that the hydrocarbon chain length of the fatty acid has a significant effect on the breakdown, or transition temperature, which denotes the passage into poor lubrication. However, there are other dependencies which govern the effectiveness of lubrication by fatty acid films. There is a significant difference between soap films formed as a reaction between the fatty acid molecule and metallic surface, and those formed simply by a physical adsorption process. Chemisorbed films were shown to lubricate in conditions where excess paraffin oil was present, conditions where the lubrication of physisorbed films often failed. Initial attempts to analyse surface films for information regarding their structure and orientation were conducted by Thomson and Cochrane (1939) and Finch and Wilman (1937) using an electron diffraction technique. Results of the experiments suggested that when the orientation of a fatty acid molecule on a metal substrate is very well defined it means that chemical action has occurred at the surface resulting in the formation of a soap.

As well as their excellent ability at reducing friction of sliding components in the boundary lubrication regime, theories have been discussed detailing another way in which metallic soap may affect the frictional behaviour of sliding surfaces (Bowden and Tabor 1950). At higher sliding speeds the formation of a viscous soap film of appreciable thickness was thought to lead to relatively large separation of the 
surfaces even at speeds well below those at which hydrodynamic lubrication might be expected to begin. Research conducted in 1951 also touches on this subject concluding that tests indicate that the rheological properties of the lubricants may have considerable influence on the frictional behaviour, and that the chemical nature of the lubricant may only be important in so far as it governs the rheological properties of any products of reaction with the surface (Barwell and Milne 1951).

The need to find friction modifier additives which conform to the CHON or NOCH philosophy, which states they must only consist of carbon, hydrogen, oxygen and nitrogen, has generated some recent publications detailing investigation into fatty acids as automotive friction modifiers. The need for a replacement to MoDTC has formed the basis for much recent research which begins to tackle the same problems considered by Bowden and Tabor in the 1950's. Work conducted at the end of the 1990s began to investigate the nature of the surfactant-solid bond and investigated thick-film and thin-film or monolayer lubrication by fatty acids (Anghel, Bovington et al. 1999). The relatively modern techniques of ultrathin-film interferometry were used to assess the contribution of surfactant type friction modifiers to film formation in high-pressure rolling, and rolling-sliding lubricated contacts. Results showed films up to $50-70 \mathrm{~nm}$ thick can be formed in slow speed contacts, suggesting the presence of a highly viscous surface layer on the rolling surfaces. Research concluded that these films were removed under conditions of high speed, ultimately concluding that additives which form thick boundary films can play a role in reducing friction in the intermediate speed range.

Research conducted in 2002 attempted to directly tackle the issues of film formation surrounding a commercial copper carboxylate additive. Tests conducted under rolling conditions showed the deposition of a thick $(\sim 70 \mathrm{~nm})$ highly viscous soap-like film on the rubbing tracks. It is reported that an interesting feature of the film was that it could be dislodged from the surfaces by high shear stress conditions, and would take time to reform. However, the paper also notes that some simple fatty acid additives were shown to form only monolayers under rolling conditions, and that only when a chemical reaction with the surface producing a soap occurs can a thick film be generated (Spikes 2002).

A commercially orientated piece of research published in 2003 evaluated a wide range of fatty acids, glycerol based molecules, amides and amines as well as MoDTC under elastohydrodynamic and boundary lubrication regime conditions (Castle and Bovington 2003). The work conducted in this study gives further foundation to the early work conducted by Bowden and Tabor, but goes further to characterise the critical performance issues of the molecules. Findings show that measured boundary coefficients of friction measured with fatty acid friction 
modifiers decrease with increasing chain length, unsaturation level and concentration, Figure 1-9.

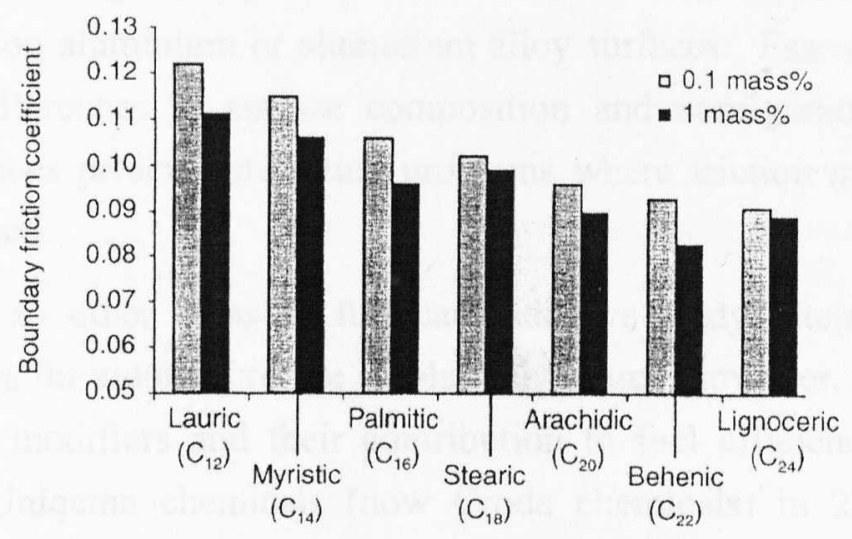

Figure 1-9 Measured Boundary Coefficient of Friction of Fatty Acids

(Castle and Bovington 2003)

Work on the glycerol mono-, di- and trioleate molecules showed that the monoester gave the best performance and that nitrogen containing friction (amide / amine) modifiers were shown to perform averagely with respect to other additives tested. Interestingly, the performance of MoDTC was considerably better than any other additive tested and puts into perspective the potency of metal complex compounds when compared to their purely organic counterparts.

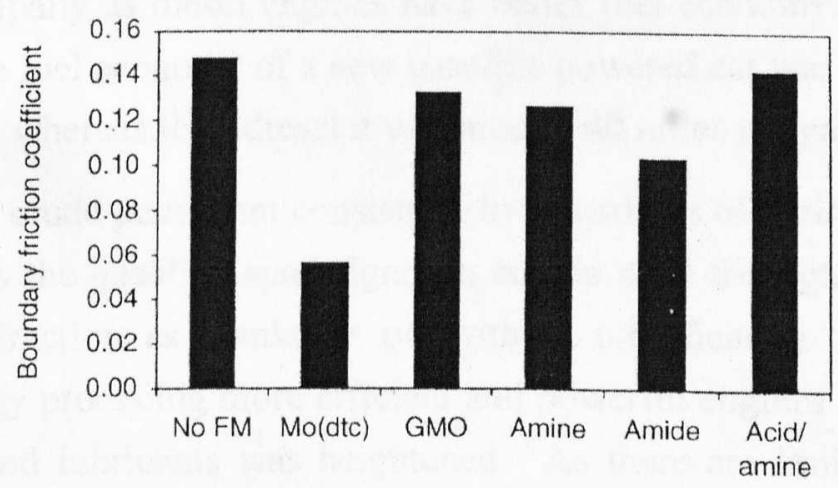

Figure 1-10 Measured Boundary Coefficient of Friction Modifiers

(Castle and Bovington 2003)

Perhaps in response to proposed future technologies, an interesting paper was written in 2004 which describes the tribochemistry of aluminium and aluminium alloy systems lubricated with liquids containing alcohol or amine additive types (Kajdas and Liu 2004). The paper reviews various 'environmentally friendly' 
lubricant additives, and makes reference to the need to find ways of lubricating aluminium and alloy systems with additive molecules conforming to the 'ecologically benign' CHON additive group. Much of the CHON additive work conducted on steel engineering surfaces is discussed with respect to applying the same principles on aluminium or aluminium alloy surfaces. Essentially the authors observe that differences in surface composition and configuration of steel and aluminium surfaces present interesting problems where friction modifier additives are to be employed.

Compared to other areas of lubricant additive study, literature on organic friction modifiers for automotive use is relatively scarce, however, a good review of organic friction modifiers and their contribution to fuel efficiency was given by researchers at Uniqema chemicals (now Croda chemicals) in 2000. The main categories of organic and non-organic friction modifiers are given with regard to their chemistry, mode of action, type and factors influencing their friction reducing properties. Interestingly the paper concludes that organic friction modifier additives and Molybdenum dithiocarbamate may act in different regimes and at different temperature conditions, suggesting a combination of the two could lead to very low coefficient of friction films (Kenbeek, Buenemann et al. 2000).

\subsection{Gasoline and Gasoline Additives}

The gasoline powered motor vehicle still significantly dominates the roads of Britain, although diesel technology is becoming ever more popular with the consumer, principally as diesel engines have better fuel economy. For example, in 2005 the average fuel economy of a new gasoline powered car was approximately 32 miles per gallon, whereas for a diesel it was around 40 miles per gallon (DfT 2006).

Essentially crude petroleum consists of hydrocarbons of various boiling points. In the early days the gasoline spark ignition engine used the light fraction as fuel, and the heavy fraction as crankcase oil without modification. With increasing engine technology producing more efficient and powerful engines the need for more complex fuels and lubricants was heightened. As there are limits to the level of improvement capable through selection and modification of the hydrocarbon structures, additives were introduced to gasoline.

In 1977 it was thought that there were really only two ways in which a gasoline additive could increase the fuel economy of the vehicle; by increasing the calorific value of the fuel, or increasing the efficiency with which chemical energy of the fuel is converted to useful work (Robinson 1977). 


\begin{tabular}{|c|c|c|c|}
\hline Additive Type & Date & Function & Compound \\
\hline Anti-knock & 1926 & $\begin{array}{l}\text { Improve } \\
\text { combustion }\end{array}$ & Tetraethyl Lead \\
\hline Anti-oxidant & 1930 & $\begin{array}{l}\text { Prevent oxidation } \\
\text { in storage }\end{array}$ & Alkyl phenol \\
\hline Anti-icing & 1952 & $\begin{array}{l}\text { Resist carburettor } \\
\text { icing }\end{array}$ & Isopropyl alcohol \\
\hline Ignition control & 1954 & $\begin{array}{c}\text { Control } \\
\text { combustion } \\
\text { chamber deposits }\end{array}$ & Tritolyl phosphate \\
\hline $\begin{array}{l}\text { Inlet System } \\
\text { Cleanliness }\end{array}$ & 1956 & $\begin{array}{l}\text { Control inlet } \\
\text { deposits }\end{array}$ & $\begin{array}{l}\text { Alkyl amine } \\
\text { phosphate }\end{array}$ \\
\hline $\begin{array}{l}\text { Inlet System } \\
\text { Cleanliness II }\end{array}$ & 1971 & $\begin{array}{l}\text { Control inlet } \\
\text { deposits }\end{array}$ & $\begin{array}{c}\text { Polyisobutene } \\
\text { amine with mineral } \\
\text { oil carrier. }\end{array}$ \\
\hline
\end{tabular}

Table 1-2 Main Classes of Gasoline Additive in use from 1926 - 1971.

After (Robinson 1977).

The additives described in Table 1-2 work mainly to ensure the engine combustion process maintains as good efficiency as is possible, and as such may be considered to maintain the efficiency with which chemical energy from the fuel is converted to useful work, with the slight exception of anti-knock additives which not only protect the engine from damage caused by the combustion process, but may allow the use of more powerful fuels and engine configurations. The authors present their views on claims made by inventors of fuel additives that can improve fuel economy, which in the context of this thesis are thought to be of significant interest:

1. The product may be entirely bogus.

2. It will, if it is valid, probably result in small benefit (say $5 \%$ at most) compared with benefits from mechanical modifications.

3. It may depend for its action on the effective weakening of mixture strength (e.g. fuel diluters such as water), which can be conducted more effectively by mechanical means.

4. It may maintain the engine inlet system in a clean condition or even clean up a dirty one.

5. It may have deleterious side effects both in the short and long term. 
6. It may really be a power-enhancing additive such as methanol.

7. It may be claimed to give 'smoother combustion', but such flamecontrolling additives are most unlikely to be found for spark ignition engines.

8. It might indeed give a real benefit by some as yet poorly realised and understood mechanism.

By 1986 it was recognised that gasoline additives needed to be formulated with completely ashless components to prevent catalyst poisoning (Tupa and Dorer 1986). In the paper it is reported that additives were used in both gasoline and diesel fuels to assure satisfactory performance in engines, protect against contamination during transport and to maintain the quality of the product until used by the end user. Additives were therefore mainly used by refineries as an economical and convenient way of achieving performance goals and a flexible, rapid way of controlling product quality, although performance additives were also on the market and included detergents, dispersants, fluidiser oils, anti-icers, combustion modifiers and flow improvers. Around 1986 OEMs began to switch from carburettor to injector inlet designs, which focused much of the work in the area on engine cleanliness, firstly to control injector deposits, and secondly to prevent valve deposits (Gibbs 1990). By 1990 , the report estimates that $40 \%$ of the gasolines on the market contained some level of deposit control additive and that most of the rest still had carburettor / injector detergents. However, as much as $10 \%$ of the gasolines on the market contained ineffective additive treatment, or no additive treatment at all. Interestingly, at this point the additives were injected at the distribution terminals, directly into the delivery tankers for delivery to service stations. By injecting directly into the tankers specific customer needs could be accounted for catering for the need to differentiate between regular and premium fuels. Gibbs went on to conclude that if an oil company decided to make a top-quality gasoline, the path was clear:

"Today's additive technology can improve the performance of any gasoline tremendously. Given the right commitment, careful selection of the right additives and the right follow through, the end result will be a superb product." (Gibbs 1990)

A review of the evolution and use of fuel additives (Danilov 2001) charts the patenting of different types of fuel additive between 1981 - 2000, Figure 1-11.

The category labelled 'other' on Figure 1-11 refer to additives such as antifoamers and additives which reduce losses in evaporation of gasoline. It is clear from the figure that gasoline additive research up to 2000 was strongly focused on 
gasoline additives as a means of maintaining the efficiency of the engine and did not attempt to increase it.

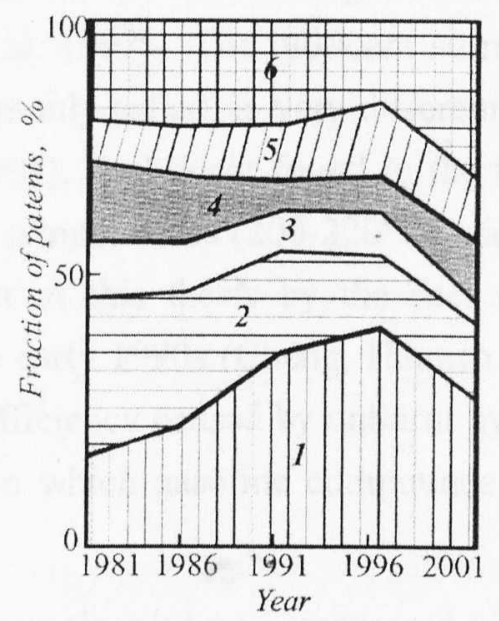

Figure 1-11 Patenting of Different Types of Fuel Additives Between 1981 and 2000: 1) detergents; 2) depressants and wax dispersants; 3) antioxidants, metal deactivators, stabilisers; 4) ignition modifier; 5) combustion modifiers; 6) other.

(Danilov 2001).

From the perspective of the automotive industry it is said that for many additives the speed of development does not appear to be very high. Many additives are used for long periods of time, sometimes for too long a time. This is partly due to the expense involved in testing, but constant innovation is required too, with the cost/performance ratio and environmental compatibility needing to be optimised at the same time. Automotive manufacturers continue to cry out for new quality additives to improve their products. Max Gairing, Senior Manager of Operational Fluids for Mercedes-Benz commented that 'fuel additives are like alloying agents in metal, they are both essential and indispensable' (Gairing 1995).

\subsection{Fuel and Lubricant Interactions}

Throughout most research institutes in the world, research into engine tribology and combustion sciences are separated. However, there are areas in the engine where an appreciation of the interaction of the combustion process and the tribology of the engine is necessary. This thesis presents research from a tribologists perspective, but utilises the excellent research conducted by combustion scientists to gain a thorough understanding of the problem environment. 
The top compression ring acts as a seal between the combustion chamber and crankcase. Changes in the design of this ring, and others in the pack, are continually made to reduce emissions caused by lubricant reaching the combustion chamber resulting in reduced oil-film thickness and longer residence times in the top piston ring zone (Fox, Picken et al. 1997). The lubricant surrounding the top ring, or the top ring zone as it is commonly called, is very different to that found in the engine sump (Bush, Fox et al. 1991). Lubricant found in the top ring zone is affected by many factors such as high temperatures $\left(200-220^{\circ} \mathrm{C}\right)$ and high shear rates, but more importantly in the context of this thesis by the fuel with which it can interact. Research conducted in the early 1990s (Cheng, Hamrin et al. 1993; Shin, Cheng et al. 1994) discusses the inefficiency caused by unburnt hydrocarbons (fuel), the latter states a number of ways in which gasoline compounds can escape burning during normal combustion.

1. Gasoline vapour-air mixture compressed into the combustion chamber crevice volumes.

2. Gasoline compounds absorbed in oil layers on the cylinder liner.

3. Gasoline adsorbed by, and/or contained within, deposits on the cylinder head and piston crown.

4. Quench layers on the combustion chamber wall left as the flame extinguishes close to the walls.

5. Gasoline vapour-air mixture left unburned when the flame extinguishes prior to reaching the walls.

6. Liquid gasoline within the cylinder that does not evaporate and mix with sufficient air to burn prior to the end of combustion.

7. Leakage of unburned mixture through the (nominally) closed exhaust valve.

The relative importance of the various sources of unburnt hydrocarbons in terms of their contribution to emissions are reported to be: $38 \%$ oil layers and deposits, flame quenching, $5 \%$ in cylinder liquid fuel effects $20 \%$ and exhaust valve leakage less than 7\% (Shin, Cheng et al. 1994). All of these mechanisms provide an opportunity for the fuel to interact with the top ring zone lubricant, and the primary source is that which directly relates the mixing of gasoline fuel with cylinder liner lubricant. The adsorption of fuel into the cylinder wall oil film takes place during the induction stroke, where tumble and swirl dynamics designed into intake systems ensure conditions in the cylinder are turbulent and the fuel-air charge is well mixed prior to ignition. This process ensures significant contact between the fuel and the 
cylinder liner oil film. When the combustion event occurs the fuel present in the oil film does not burn. After combustion, since the mass fraction of fuel in the combustion chamber will have dropped to zero, and the temperature risen, the fuel in the film will begin to diffuse out into the combustion chamber. Some research reports that increasing engine speed may increase the oil-film thickness sufficiently for there to be an increase in the amount of fuel desorbed from the oil film, but that at the highest engine speeds this may not be true due to the lack of time available for the diffusion to occur, thus resulting in significant concentrations of unburnt fuel in the cylinder liner oil-film (Thompson, Radcliffe et al. 1997).

Several studies have been conducted to extract lubricant from directly behind the top ring, in the top ring zone, to investigate the phenomenon of fuel dilution in the cylinder liner oil film. The technique of top ring zone sampling during firing engine conditions plays a significant part in this thesis and is discussed in detail in the following chapter. A study conducted by PSA/Peugeot/Citroen in 1996 looked at the effects of gasoline boiling point on individual fuel hydrocarbon species absorbed in the oil and found that they increase exponentially with boiling point. The study concludes that the rate of hydrocarbon build-up in the oil film is initially faster that the accumulation of fuel in the sump, but lower final concentrations are reached and the ratio of fuel to lubricant can be directly correlated with the boiling point of the fuel species (Frottier, Heywood et al. 1996).

Research conducted in 1997 by the Massachusetts Institute of Technology investigated the presence of liquid fuel in the cylinder of a firing port-injected gasoline engine during start-up, where the injected fuel is enriched to provide a significantly large amount of vaporised fuel, so that smooth starting is achieved. Again the study reports that a large portion of the excess fuel does not vaporise and enters the cylinder as liquid. The fuel is then stored in various places in the cylinder, such as in the oil layers, where it survives the combustion event unburned (Meyer and Heywood 1997). The study discusses that 'wall wetting', the process in which fuel directly interacts with the walls, is extremely dependent on valve timing where an indirect injection system is used. In regions which are more exposed to the spray path of the injector, more significant wall wetting occurs. This theory was further investigated by researchers at Wayne State University who used colour image capturing techniques to visually assess cylinder liner wall wetting as a function of engine speed, injection timing and injector type, again during the engine start up process (Kim, Yoon et al. 2003). One of the images captured in the research shows the extent of the fuel 'footprint' caused through the impingement of fuel droplets on the cylinder liner oil film, Figure 1-12. 


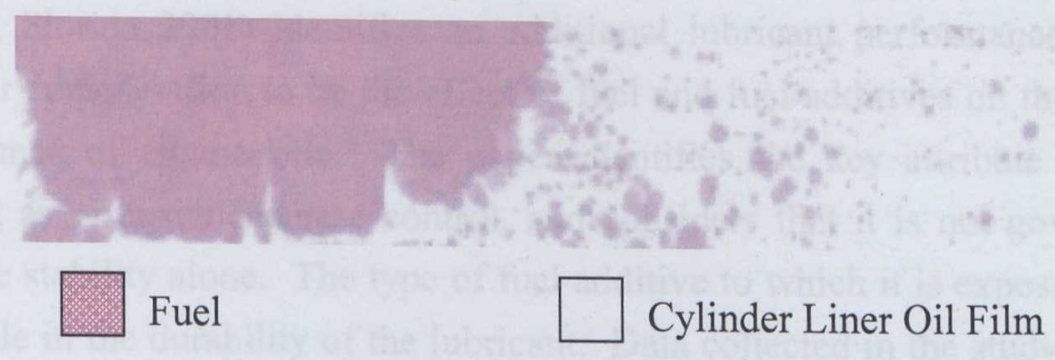

Figure 1-12 Image of Fuel Dilution in the Cylinder Liner Oil Film.

After (Kim, Yoon et al. 2003).

Fuel contamination of the cylinder liner / piston assembly lubricant is not only a phenomenon which is present at start up engine conditions, top ring zone sampling conducted at the University of Leeds during steady state firing conditions has measured up to $80 \%(\mathrm{~m} / \mathrm{m})$ fuel dilution in top ring zone samples (Gamble 2002a).

It is clear then that the lubrication of the piston assembly is heavily affected by the combustion process, and that any attempt to reduce piston assembly friction as a means of increasing fuel economy should consider the inevitable interaction between fuel and lubricant components in the combustion chamber. Despite attempts to restrict lubricant fuel contamination by both combustion scientists and tribologists, as it is considered a detrimental phenomenon by both parties, it is generally accepted to be an inevitability.

Commercially orientated work conducted by researchers at Texaco Additives International recognised the interaction between fuel and lubricant and used the fuel itself to deliver friction modifier to the piston ring / cylinder wall interface, a location where friction is high and oil quantity kept purposely low (Hayden, Ropes et al. 2001). The research, and certainly the publication of this type of work, conducted by this company may be considered to be seminal in the field. As such, details of the system are hidden due to its commercial sensitivity, but it is discussed that the concept utilises the intake charge tumble and swirl systems designed into modern engines to distribute heavy additive molecules of fuel additive to the cylinder wall. Research showed the system to be instantaneously effective at reducing friction and that the friction reduction increased with time. The fuel economy benefits were independent of engine age or mileage, deposit level, model or brand although a decreased benefit was seen where engine oil friction modifiers were already employed and where high viscosity engine oils were used. Maximum fuel economy benefits of $4.66 \%$ were quoted to be achievable through the application of this type of additive technology.

A sister publication (Thiel and Hayden 2001b) presented at the same conference (SAE International Spring Fuels \& Lubricants meeting and exhibition, 
Orlando, Florida 2001) identifies an additional lubricant performance influencing factor for consideration to be the effect of fuel and fuel additives on the quality and performance of engine oils. The paper identifies the key attribute needed in a lubricant as viscosity increase control, and considers that it is not governed by its oxidative stability alone. The type of fuel additive to which it is exposed can play a major role in the durability of the lubricant. Data collected in the study showed that certain types of fuel additive can form a gel-like matrix with lubricant containing high saturate base stocks once the oil's dispersant package is depleted, Figure 1-13. Another class of fuel additive was shown to impede the function of the lubricant's antioxidant system. It is suggested that the additive ties up the radical scavengers and peroxide decomposers in the motor oil making the base oil more prone to oxidation, thus reducing the service life.

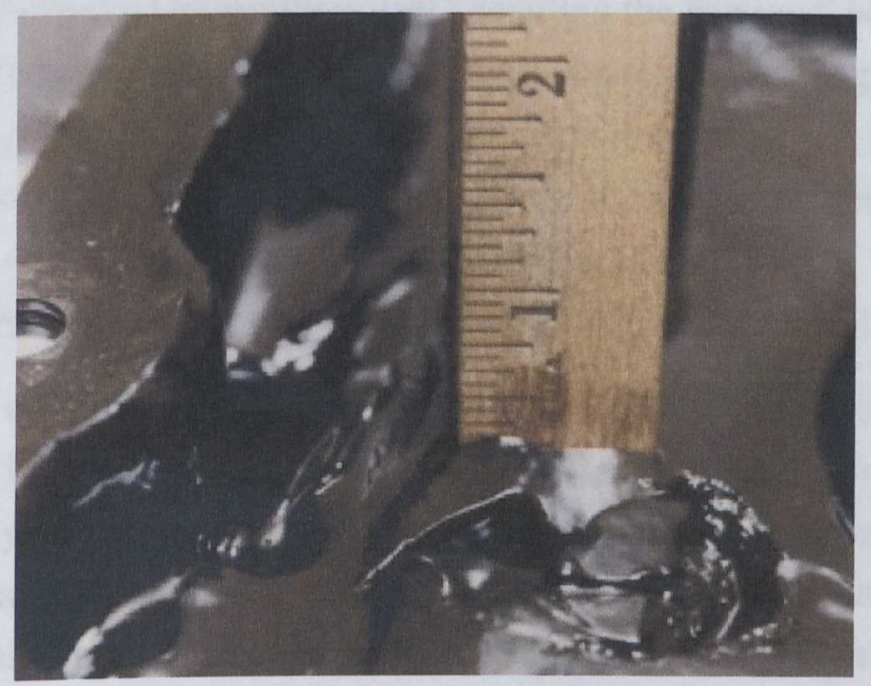

Figure 1-13 'Gel-Like' Sludge Formation in Engine Sump as a Result of Incompatibility Between Lubricant and Fuel Additive (Thiel and Hayden 2001b)

\subsection{Other Attempts at Increasing Fuel Economy}

Within the automotive industry a broad and diverse range of research is conducted in pursuit of making the motor vehicle more fuel efficient. The purpose of this section is to highlight some of the interesting research conducted to increase fuel economy.

The desire and need to improve automotive fuel economy has stimulated work in engine parametric optimisation, drive line and transmission development and vehicle weight reduction programs. Knowledge of the use and drive cycles of automotive vehicles have also been used to highlight specific areas in which fuel 
consumption savings can be made. In 1979 it was estimated that approximately $30 \%$ of the automotive fuel consumed in the United States was spent on trips of 5 miles or less (Marshall 1979). Fuel economy after engine start up is markedly lower than the fully warmed up condition, primarily due to the need for enriched mixture conditions as discussed in previous sections. Studies have shown that for a 5 mile trip from cold start, vehicles achieved only $80-90 \%$ of their fully warmed up economy. With low ambient temperature conditions this economy is reduced even further to just $60-70 \%$ efficiency. With this knowledge in mind researchers investigated ways of reducing the duration of enrichment, quoting that an increase of $5 \%$ in fuel economy during the warm up cycle would cause a decrease in gasoline consumption of about 1 billion gallons per year. Work also focused on reformulating fuels with better volatility characteristics although no real success was reported.

A study by Fukuoka University in 1992 detailing the frictional behaviour of the piston assembly through both numerical and experimental investigation recognised that decreasing piston ring friction was an important way of improving the mechanical efficiency of the engine. They broadly state that for a piston ring pack with five piston rings, as a means of reducing the friction loss it is effective to simply decrease the number of piston rings (Wakuri, Hamatake et al. 1992).

In 1995 a number of publications were released documenting the use of polytetrafluorethylene (PTFE), packaged as 'Slick 50', as a friction reducing additive, primarily in response to the need for zinc and phosphorus-free (ashless) additives to replace ZDDPs and MoDTC. The paper claims that the additive modifies the metallurgy of the engine surfaces with which it comes into contact with makes it more of a surface engineering treatment than a lubricant additive (Wilson 1995).

Various engine manufacturers and research institutes have investigated the effect of crankshaft offset on piston friction side force in the gasoline engine. Research published in the 2000's explains that a major automotive manufacturer had already put the technology in a production engine to improve fuel economy. The research set about to clarify exactly how offsetting the crankshaft axis with respect to the cylinder axis modifies the frictional behaviour of the piston assembly. The study concluded that the effect of crankshaft offset on piston friction could not be explained only by the effect on the side force acting on the piston. Changes in the piston skirt contact and oil film development complicated matters and were also thought to affect the piston friction characteristics (Nakayama, Tamaki et al. 2000; Wakabayashi, Takiguchi et al. 2003). 
Of course energy savings can also be made through improving other important areas of the engine. A study by Ford discusses attempts to reduce frictional losses in direct-acting mechanical bucket tappet type valvetrains, an area which typically contributes $6-10 \%$ of the total frictional loss in an engine, through use of modified surface finish, surface texture and coatings. The research observed that surface finish and surface texture could reduce frictional losses, but that engine oil formulation also plays a significant role (Gangopadhyay, Soltis et al. 2004).

An extremely novel approach to reducing piston assembly friction by the Nissan motor company was published in 2005 . The bores of the cylinder block are usually machined prior to assembly with the cylinder head. However, this results in bore distortion when the cylinder block is assembled with the cylinder head due to the load applied by the head bolts and the surface pressure of the head gasket. This bore distortion influences the effectiveness of the seal between the piston assembly and cylinder liner walls, requiring an increase in bore thickness and addition of manufacturing ribs to obtain greater cylinder block rigidity which leads to an increase in weight. A novel manufacturing process was used to machine the bores when fitted with a dummy cylinder head ensuring bore circularity with weight savings, the latter inherently forcing greater vehicle economy. The greater bore conformity allows the piston ring index (total ring tangential load / bore diameter) to be reduced by $50 \%$. This has the knock-on effect of reducing friction by approximately $6.6 \%$, resulting in a fuel economy improvement of $1.3 \%$ (Matsuo, Kiga et al. 2005).

At a seminar of the European Parliament which took place early in 2006, a number of innovations that could help bring down emissions were discussed (BBC News and Mulvey 2006). Among them were:

- Better streamlining - including dashboard displays to take the place of wing mirrors.

- lights on the dashboard to indicate the best moment to change gear.

- making cars lighter, for example by modifying the seat structures.

Changes to engines could include:

- A device to stop the engine when the car comes to halt and start it again with a press on the accelerator.

- More hybrid cars, which run partly on electricity.

- Further moves towards efficient diesel engines.

- reducing the top speed of cars, to bring it nearer to the maximum speed they are likely to be driven at on European roads. 


\subsection{Overall Strategy of the Project}

This thesis describes a seminal piece of research which bridges the gap between chemistry, surface science, materials science, engineering and tribology as it is believed that this multidisciplined approach is essential when tackling vastly complex problems such as furthering the fuel economy of the gasoline engine. In concert with an appreciation of all sciences underpinning the operation of the gasoline engine, logic, creativity and innovation were employed to both create and achieve the key objectives outlined below.

- Experimentally assess fuel dilution in the piston assembly lubrication system of a firing gasoline engine operating under varying engine conditions.

- Quantitatively investigate the mechanism through which gasoline friction modifier can be administered to the piston assembly lubrication system of a firing gasoline engine.

- Develop a bench-top test to screen the performance of environmentally friendly friction modifiers under in-cylinder boundary lubrication conditions, generating information about the capability of gasoline administered CHON friction modifiers to reduce piston assembly friction and aiding the development of bespoke formulations for further investigation.

- Investigate the mechanism of friction reduction brought about by the use of CHON friction modifiers through application of a number of sophisticated surface analysis techniques.

- To develop refined technologies to assess the ability and mechanism of action of gasoline containing friction modifier to reduce piston assembly friction in a firing gasoline engine.

Due to the seminal nature of this project, each of the above objectives were considered as guidelines for investigation and were expanded upon during the natural evolution of the research.

\subsection{Summary}

In this chapter, discussion identified the need for improvement in the fuel economy of the motor vehicle, whose popularity and dependence on hydrocarbon fuels causes damage to the world we live in. An overview of the process through which legislation is created to govern the development of the motor vehicle its fuels 
and lubricants is given. Attention focussed on the mechanical losses associated with the operation of the gasoline engine, detailing the evolution of the fuel efficient lubricant and its constituent additive components. Problems with current additives which make possible current fuel economy savings and have a massive dominance in the marketplace were discussed, and the need for their replacement identified. Organic replacements for the metal based additive MoDTC responsible for friction reduction in most fully formulated lubricants were reviewed.

The gasoline powered vehicle and gasoline additive technology over the last 30 years was discussed detailing the dominance of detergent systems to maintain engine efficiency. Research was reviewed presenting the interaction of gasoline fuel and engine lubricant in the combustion chamber leading to the proposition of this thesis which investigates in-cylinder fuel and lubricant effects to reduce gasoline engine friction. 


\section{In-Cylinder Lubricant Sampling}

In Chapter 1 it was stated that fuel entering the engine lubricant can have a significant effect on the operational behaviour of the piston assembly. It is this fuel dilution in the cylinder liner lubricant that forms the foundation to the hypothesis suggesting friction modifier can be administered to the piston assembly/cylinder liner interface via the fuel.

A series of engine tests was developed to further investigate the lubrication environment of the piston assembly. This portfolio designed as a set of 'scouting tests', whose purpose was to broadly investigate piston assembly lubrication conditions with varying engine condition. Specific tests were designed to investigate both the lubricant which acts directly at the Top Ring Zone (TRZ), as well as the lubricant down the cylinder liner wall. Of specific interest were the levels of fuel dilution found in lubricant extracted from these locations and the potential additive concentrations which may accumulate in these areas, achieved through doping the engine fuel with an appropriate marker.

\subsection{The Ricardo Hydra Gasoline Engine}

A Ricardo Hydra gasoline engine housed at the Institute of Tribology Engine Tribology Research Laboratory, The University of Leeds, was used in this research, Figure 2-1. The engine is manufactured by Ricardo Consulting Engineers Ltd in the $\mathrm{UK}$, and is available in a variety of configurations. The chosen engine has a single cylinder naturally aspirated configuration, with 2 valves per cylinder and indirect gasoline injection. The bore/stroke arrangement of the engine is $86 \times 86$ (bore $(\mathrm{mm})$ $\mathrm{x}$ stroke(mm)), based directly on a General Motors 2.0 Litre, 4 cylinder production engine which was first available on the market in 1988. There are several features which made the Hydra engine ideal for this research:

- The engine has a separate control module in place of the typical Engine Control Unit (ECU), with individual control of engine speed, ignition timing and fuel injection, thus permitting the simulation of numerous engine conditions with ease.

- The engine incorporates a split sump design which separates the lubrication of the valve train and crankcase. This is considered advantageous as it allows the two systems to decoupled. 
- As it is designed for the laboratory rather than for a vehicle, the overall geometric configuration of the engine is less constrained than that of a typical production engine, and lends itself to easy access and bespoke adaptation of systems as well as the incorporation of new components.

- The engines ancillaries are all externally driven providing individual control of lubricant supply pressures and fluid temperatures.

- The engine is connected to a swinging arm dynamometer which serves to brake the engine under normal firing operation, but can also be used to motor the engine should that be required.

- The engine is fitted with a bespoke PC based monitoring system which gives the user on-line information regarding the engine speed, load and mixture as well as information regarding operating temperatures at numerous locations around the test facility. In addition to giving the user on-screen information detailing the operation of the engine, the information is recorded and processed for post test analysis.

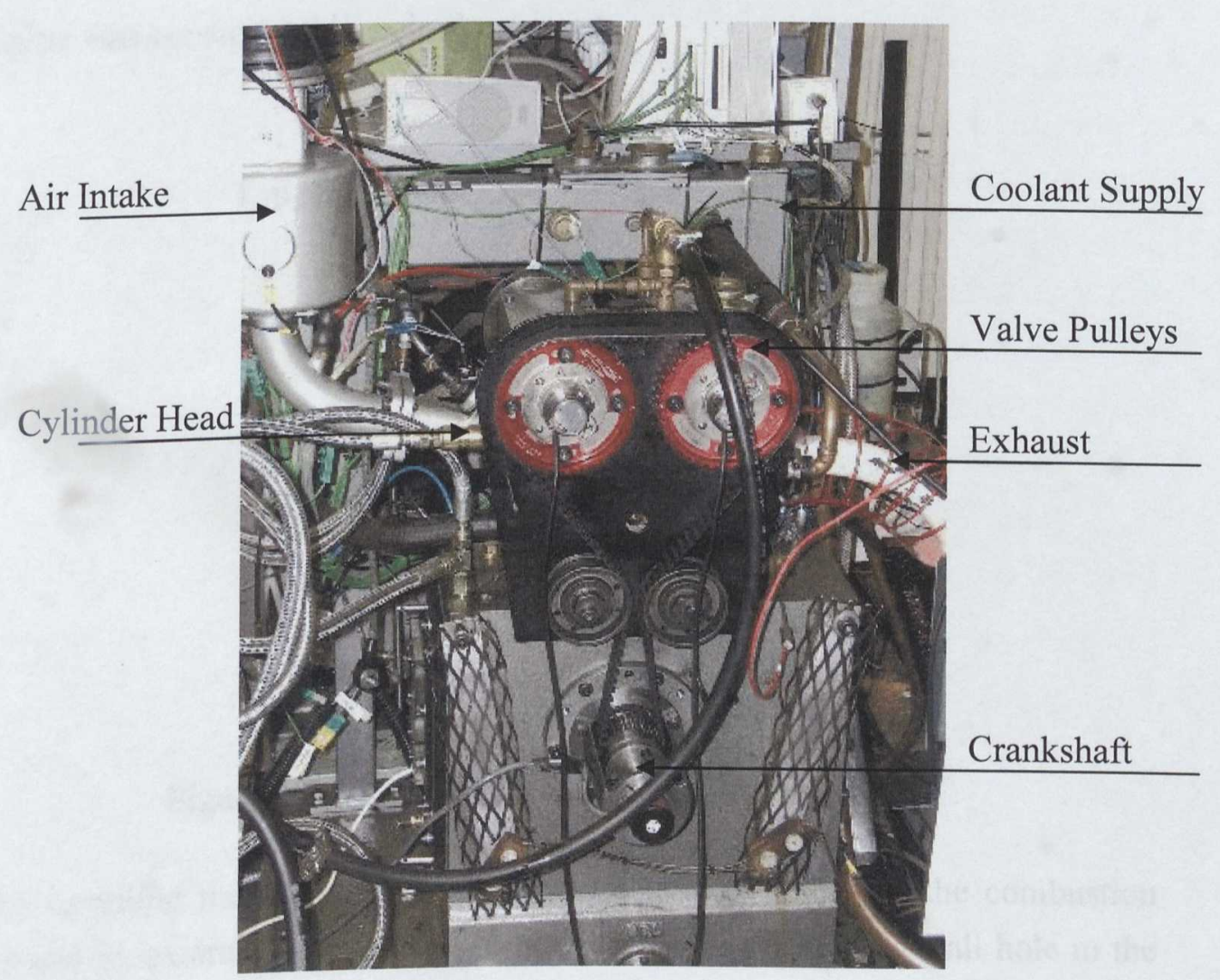

Figure 2-1 The Ricardo Hydra Research Engine 


\subsection{Top Ring Zone Sampling}

Sampling from the top ring zone is a method which has previously been used by many researchers to understand the composition, condition and effectiveness of the lubricant behind the top piston ring (Saville, Gainey et al. 1988; Bush, Fox et al. 1991; Taylor and Evans 2004). The technique is typically employed using relatively crude apparatus on heavy duty diesel engines due to their slow speed and large size. The standard TRZ sampling technique has now been developed to work in a single cylinder gasoline engine, overcoming the challenges of more restrictive geometry and higher engine speeds (Figure 2-2). The development of the original TRZ sampling system was conducted by Gamble et al 2002a. At this time the bottom-end of the system, which includes the mechanism for passing the sampling pipe out of the engine, was poorly designed and frequently broke. Work conducted in association with this research developed the bottom-end of the system to include an axial constraint which significantly improved the reliability of the apparatus permitted long test runs ( $200 \mathrm{hrs})$ at speeds of up to $2000 \mathrm{rpm}$. This development followed on from a similar system employed by Frottier et al in 1996, where the sample pipe was constrained in a similar manner.

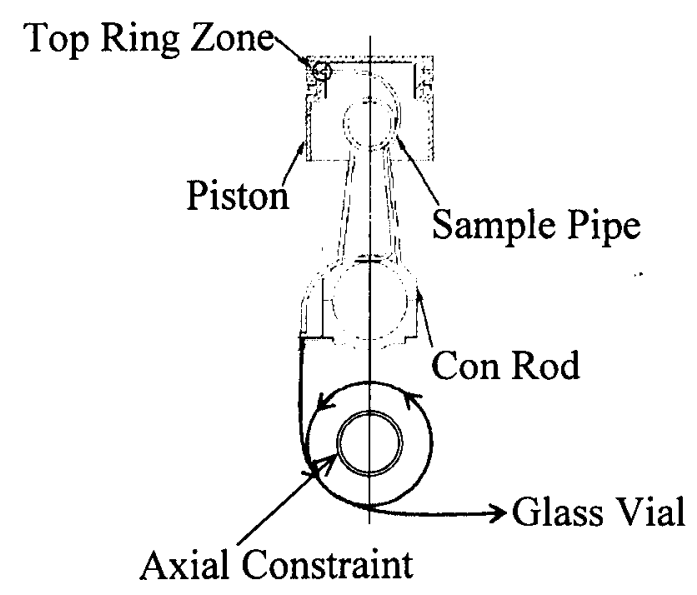

Figure 2-2 Top Ring Zone Sampling Apparatus

The sampling method utilises the pressure gradient between the combustion chamber and its external environment to push lubricant through a small hole in the back of the top ring groove. A $0.5 \mathrm{~mm}$ hole is drilled in the back of the top ring groove and connected to a robust $1 / 8^{\text {th }}$ inch $(-3.18 \mathrm{~mm}) \varnothing$ PTFE tube. This tube then enters a stainless steel sleeve, protecting its path around the connecting rod. At the base of the connecting rod the $1 / 8^{\text {th }}$ inch $(-3.18 \mathrm{~mm}) \emptyset$ tube is connected to a $3 / 16^{\text {th }}(\sim 4.76 \mathrm{~mm})$ inch $\varnothing$ PTFE tube which loops around a one-dimensional 
constraint stabilising the tubes motion and protecting it at high speed. On exiting the engine the vapour stream carrying the lubricant sample is sprayed against the side of a glass vial where it is condensed under ambient conditions and harmful gasses extracted. Early attempts used a condensation column prior to the collection vessel in a bid to minimize evaporation of the more volatile species, but this led to an excessive collection of water derived from the combustion event. The collected sample was an emulsification of water in hydrocarbon and as such the interrelation of fluids made subsequent analysis difficult.

\subsubsection{Top Ring Zone Sampling Tests}

Top ring zone sampling tests were conducted in collaboration with Dr David Barrell of the University of Leeds, and some results shared in a sister publication, (Barrell 2004).

There were two main aims for this section of the project: 1) To investigate fuel dilution in the TRZ lubricant, 2) To evaluate the possibility of administering additive to the piston assembly lubricant via the fuel. In order to do this, the bulk of the engine tests were conducted on a standard 95 octane unleaded gasoline containing a potassium marker. This marker was employed to simulate the presence of additive, and provides a mechanism for quantitatively assessing the amount of additive in a sample. Fuels were blended with varying amounts of potassium marker $(10,21$ and $42 \mathrm{mg} / \mathrm{kg}$ ) facilitating investigation into the effects of additive treatment level variation (Table 2-1). In order to investigate the effect of variation in boiling point of the fuel, a High Boiling Point fuel (HBP) containing $10 \mathrm{mg} / \mathrm{kg}$ potassium was tested in a stand-alone test. Full details of the fuels used in these tests are given in the Appendix A4. A fully formulated Shell SAE 5W-30 lubricant provided by Shell Global Solutions (UK) was used throughout all engine tests. In-between tests on different fuels a lubricant flush and oil filter change procedure ensured all traces of the previous fuel were removed from the engine.

\begin{tabular}{ccc}
\hline Base fuel & $\begin{array}{c}\text { Potassium Analysis } \\
\text { (mg/kg) }\end{array}$ & Reference Name \\
\hline SPL1995/03 & 10 & Fuel Aa \\
\hline SPL1995/03 & 21 & Fuel Ab \\
\hline SPL1995/03 & 42 & Fuel Ac \\
\hline TBR8647/03 & 10 & Fuel Ba \\
\hline
\end{tabular}

Table 2-1 Fuels Blends Used In Investigation 
Engine tests were designed to investigate the effect of changing a single engine parameter on the level of fuel and fuel additive in the top ring zone lubricant (Table 2-2). Engine parameter variables were engine speed, load, additive treatment level and air / fuel ratio. In Table 2-2 the variation of air / fuel ratio is represented using Lambda $(\lambda)$. Lambda $(\lambda)$ is a measure of how far from stoichiometry a mixture is. A Lambda of 1.0 is at stoichiometry, rich mixtures are less than 1.0 and lean mixtures more than 1.0. Throughout the tests oil temperature was monitored and thermostatically maintained at $90^{\circ} \mathrm{C} \pm 2^{\circ} \mathrm{C}$, coolant temperature at $90^{\circ} \mathrm{C} \pm 2^{\circ} \mathrm{C}$ and air inlet temperature to $30^{\circ} \mathrm{C} \pm 1^{\circ} \mathrm{C}$.

Prior to each test the engine was preheated, motored for a five minute period, and then fired until temperatures had stabilised. Once thermal equilibrium had been achieved, a vial was connected to the TRZ sampling system and sample collected for a test duration of six hours. Sample vials were changed when the sample volume exceeded $3 \mathrm{ml}$, the minimum volume required for subsequent analyses.

\begin{tabular}{|c|c|c|c|c|c|c|c|}
\hline \multirow{2}{*}{$\begin{array}{c}\text { Load } \\
(\% \text { of full })\end{array}$} & \multicolumn{7}{|c|}{ Speed (rpm) } \\
\hline & 1000 & 1500 & \multicolumn{5}{|c|}{2000} \\
\hline $100 \%$ & & & \multicolumn{5}{|c|}{ - } \\
\hline $75 \%$ & & & \multicolumn{5}{|c|}{$\bullet$} \\
\hline \multirow{6}{*}{$50 \%$} & \multirow{6}{*}{$\bullet$} & \multirow{6}{*}{$\bullet$} & Treat & \multicolumn{4}{|c|}{$\lambda$} \\
\hline & & & $(\mathrm{mg} / \mathrm{kg})$ & 0.9 & 0.95 & 1.0 & 1.1 \\
\hline & & & 10 & $\bullet$ & $\bullet$ & $\bullet$ & $\bullet$ \\
\hline & & & 21 & & & $\bullet$ & \\
\hline & & & 42 & & 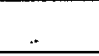 & $\bullet$ & \\
\hline & & & 10 (HBP) & & & $\bullet$ & \\
\hline $25 \%$ & & & \multicolumn{5}{|c|}{ - } \\
\hline
\end{tabular}

Table 2-2 Top Ring Zone Sampling Engine Testing Matrix

\subsection{Three Point Lubricant Sampling From The Cylinder Wall (TPS)}

Little is known about the axial variation of the cylinder liner lubricating film within a firing gasoline engine. To complement the TRZ sampling equipment a Three Point Sampling (TPS) System was developed for sampling the lubricant film directly from the cylinder liner. The design of the system was conducted using IDEAS, a Computer Aided Design (CAD) software package (Figure 2-3). CAD was selected for use in the conceptual design stages of this project as it is inherently flexible and allowed the evolution of the design to take place iteratively and with low cost. The CAD package also permitted the accurate calculation of drilling 
locations and angles ensuring manufacturing went without any problems. Further information regarding the design of the TPS system can be found in Appendix section A5.

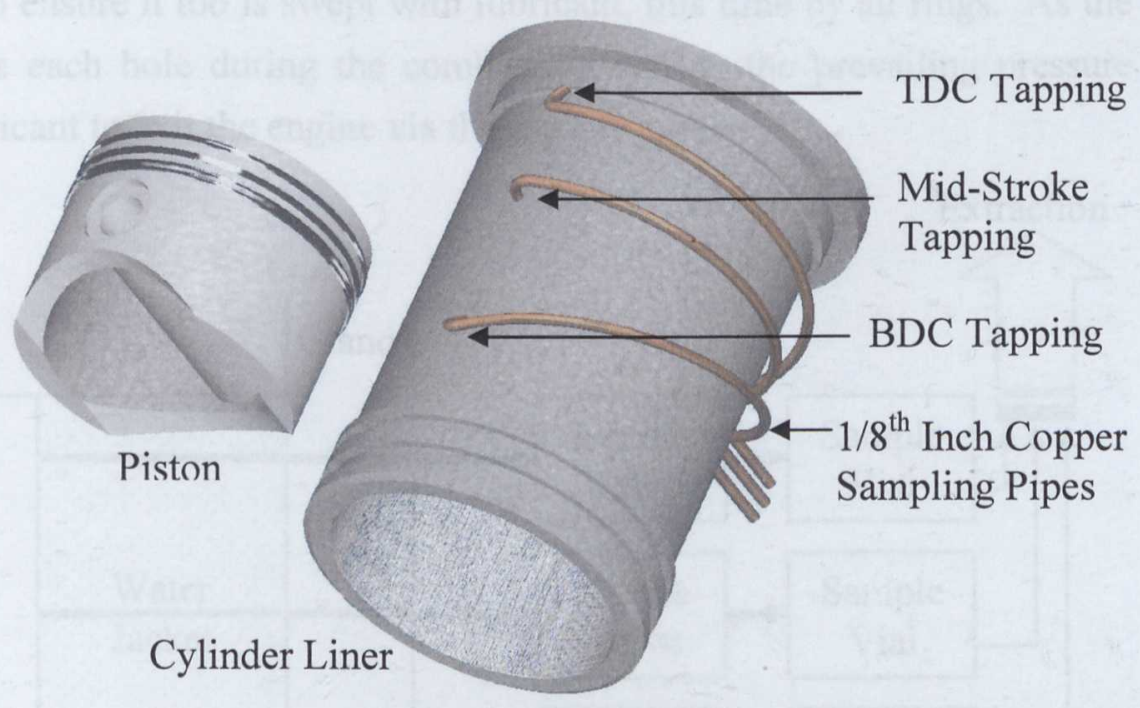

Figure 2-3 CAD Image of Three Point Sampling System

Similarly to TRZ sampling, the design of TPS system is the complicated by the restrictive geometry of the gasoline engine. However, the internal geometry of the Hydra research engine design leaves some room for the fitting of additional components. Figure 2-3 shows the locations of the sampling pipe tappings where they exit the cylinder liner wall and enter the cooling water jacket. The pipes then follow the contour of the external cylinder closely before grouping together to exit the engine.

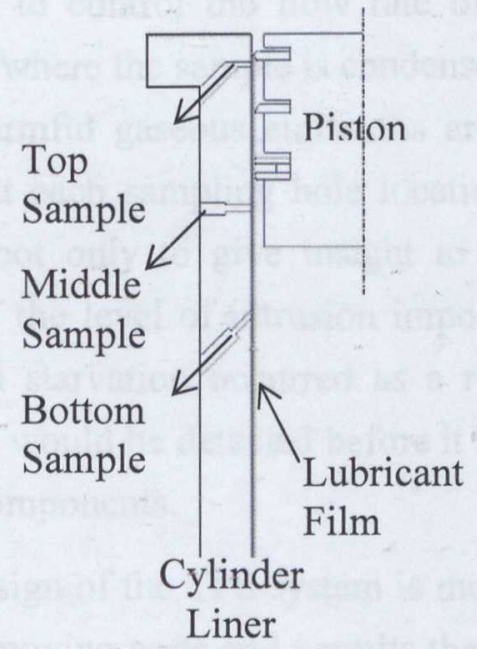

(a)

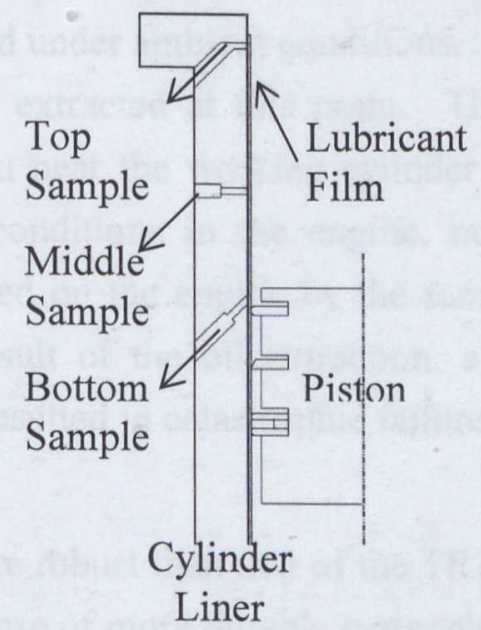

(b)

Figure 2-4 Three Point Sampling System: (a) TDC Reversal, (b) BDC Reversal 
The internal system features three $0.5 \mathrm{~mm}$ holes located at strategic locations on the thrust side of the engine (Figure 2-4). The top hole is slightly below the TDC location for the top piston ring ensuring it is swept with lubricant as the piston moves to the TDC. Similarly the bottom aperture is located above the BDC position of the top ring to ensure it too is swept with lubricant, this time by all rings. As the ring pack passes each hole during the combustion stroke, the prevailing pressure wave forces lubricant to exit the engine via the sample pipes.

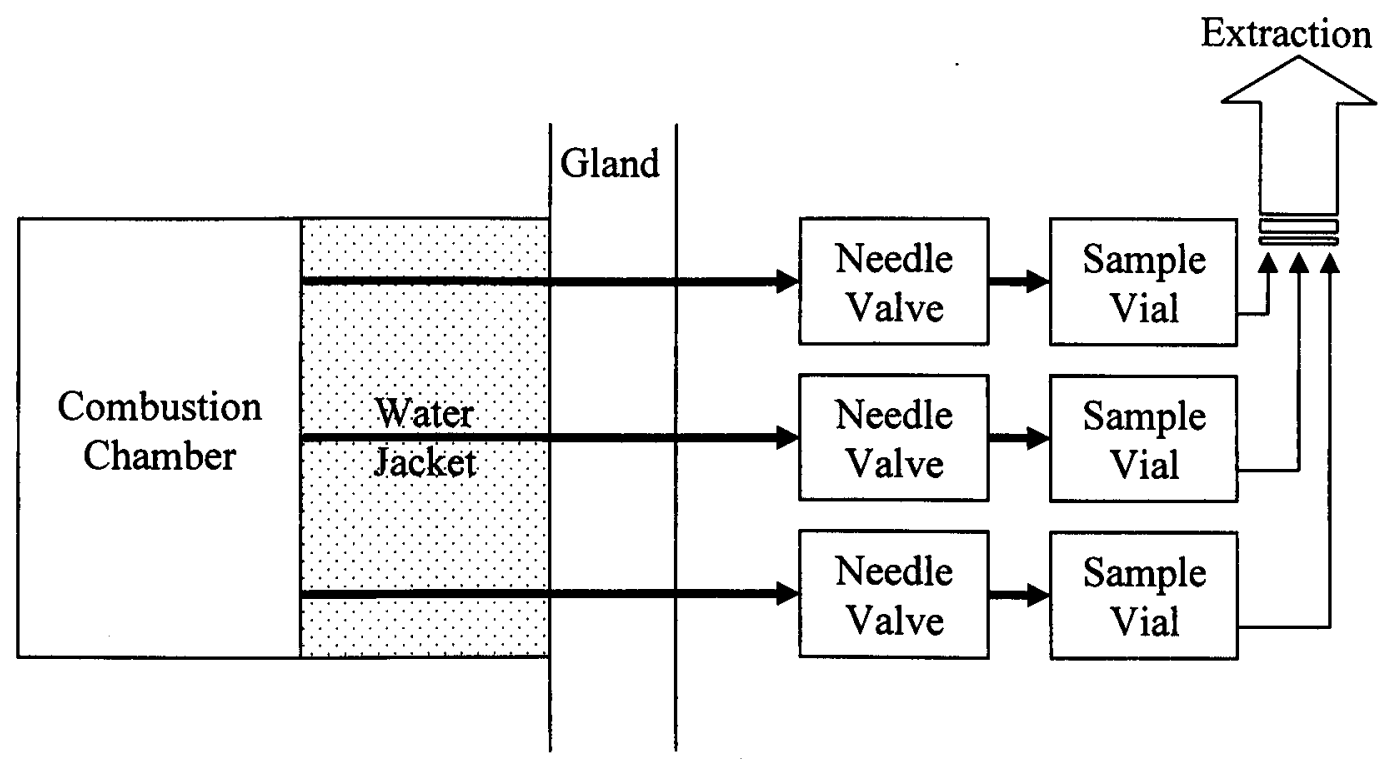

Figure 2-5 Flow Diagram of Three Point Sampling System

On exiting the combustion chamber, the sample travels down $1 / 8^{\text {th }}$ inch $(0.125$ $\mathrm{mm}$ ) copper tubes which are screwed directly into the cylinder liner material. These tubes pass through the cylinder liner cooling water jacket and exit the engine on the anti-thrust side via a gland system (Figure 2-5). Once outside the engine, needle valves serve to control the flow rate of the sample which is directed into glass sample vials where the sample is condensed under ambient conditions. As with TRZ sampling, harmful gaseous emissions are extracted at this point. Thermocouples were fitted at each sampling hole location near the working cylinder liner surface and served not only to give insight to conditions in the engine, but to give an indication of the level of intrusion imposed on the engine by the sampling system itself. If oil starvation occurred as a result of the oil extraction, a rise in liner temperatures would be detected before it resulted in catastrophic failure or seizing of the engine components.

The design of the TPS system is more robust than that of the TRZ system as it contains no moving parts and permits the use of more durable materials. As a result the system lends itself to tests at elevated speeds with respect to that of the TRZ tests. However, for this work speeds were restricted to a maximum of $3000 \mathrm{rpm}$ for 
safety reasons. It was feared that if failure of a sampling pipe occurred at elevated speed, this could result in water passing from the water jacket into the cylinder liner and subsequent rapid catastrophic failure of the engine.

\subsubsection{Development and Problems with the TPS System}

As with any bespoke system designed as a retro-fit application, the development of the TPS system involved solving many problems. During early test runs it was suspected that the mechanism of screwing the tapped copper pipes directly into the cast iron was resulting in mild leakage. Fluid transfer from the cylinder liner cooling jacket into the combustion chamber not only affects engine running conditions and contaminates samples, but is considered extremely dangerous as it could potentially result in hydraulic 'locking' of the engine. For these reasons it was decided to dope the engine coolant with a mild purple ink, the intention being that any transfer of fluid from engine coolant to sump lubricant would be detectable. Subsequent engine operation revealed that oil samples taken from the TPS system had a slight purple tint indicating some fluid transfer had occurred. As a result of this, the decision was made to strip the engine, dismantle the apparatus and coat the pipe threads in standard Araldite ${ }^{\mathrm{TM}}$, an epoxy based glue capable of withstanding the most arduous conditions. Once screwed into the cylinder liner sampling holes, the glue was left to harden for a day prior to fitting the apparatus in the engine. This glue was also used to securely fit the thermocouples in blind holes which terminated close to the cylinder liner surface, and is clearly visible in Figure 2-6.

With the problem of coolant leakage resolved, engine testing commenced without a hitch. However, after a number of hours it was noticed that the top sampling pipe was slightly blocked. To remedy this, the engine head was removed and a pneumatic line connected to the sampling pipe at the end usually connected to a sample vial. Compressed air was forced through the pipe until it dislodged the blockage, which was expelled from the system into the combustion chamber. On inspection it was clear that carbon deposits which would usually be forced out of the engine through the exhaust system were becoming lodged in the top sampling pipe, close to the sampling hole itself. This phenomenon is one which is only associated with cylinder liner sampling and is thought to be caused by the fact that the top sampling hole is exposed to the full combustion process occurring at the top of the engine cylinder, an effect not seen when top ring zone sampling. This type of blockage occurred a number of times throughout the testing series and was always remedied through the application of compressed air, a procedure which became a standard maintenance operation. However, after a number of test hours a large deposit formed in the top sampling line which could not be removed through 
application of compressed air. Several other methods of removing the blockage were employed including trying to destroy the blockage through feeding thin wire down the pipe, dissolving the deposit in solvent and application of a compressed oil line which exerted extremely high pressures behind the blockage, however the deposit was firmly lodged in place and would not be moved. This blockage of the top sampling pipe was so severe that it was necessary to cut the copper pipe open to remove the deposit, which appeared to be a large solid formation of carbon (Figure 2-6). As this occurred close to the end of the engine test series it was not deemed viable to re-instrument another cylinder liner, and the test series was terminated.

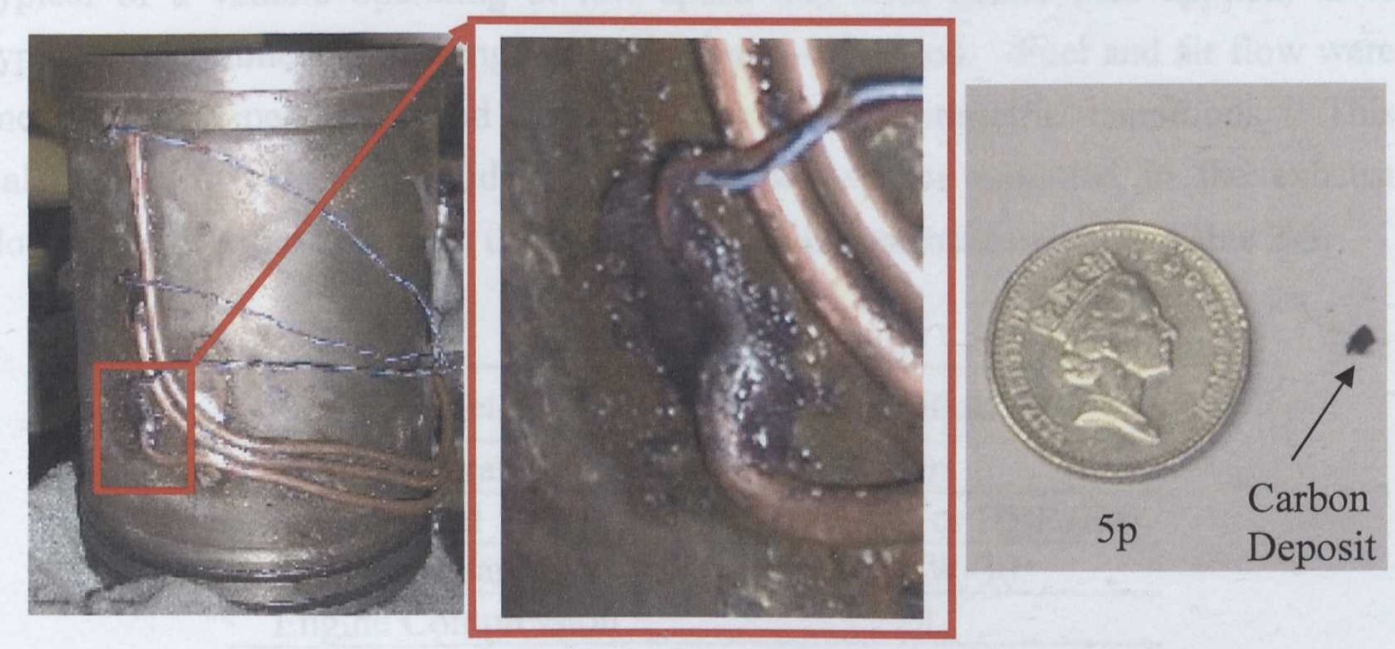

Figure 2-6 Photograph of TPS Liner, Sampling Pipe Attachment and Carbon Deposit

\subsection{Three Point Sampling Tests}

Two types of test were conducted using the Three Point Sampling (TPS) system. The first test involved starting the engine from cold, and collecting samples to give information about fuel dilution and additive concentrations in the cylinder liner oil film under start-up conditions. The second set of tests mimicked the TRZ sampling tests in that the aims were both to investigate fuel dilution and potassium concentration in the sampled lubricant with varying engine condition, although this time the lubricant under investigation was sampled from the cylinder liner oil film, and not from the top ring groove.

\subsubsection{Cold-Start Engine Tests}

The aim of the cold start tests was to investigate fuel dilution and additive concentrations in the cylinder liner oil film under warm-up conditions. The effects of the final boiling point of the fuel was also investigated in this test series through 
implementation of two fuel types selected from the portfolio of fuels tested in the $\mathrm{TRZ}$ tests, fuel $\mathrm{Ab}$ and fuel $\mathrm{Ba}$ (Table 2-1). Fuel $\mathrm{Ab}$ is a standard 95 octane gasoline with $21 \mathrm{mg} / \mathrm{kg}$ of Potassium marker, Fuel $\mathrm{Ba}$ is a slightly denser high boiling point fuel with a potassium marker level of $10 \mathrm{mg} / \mathrm{kg}$.

As the tests were conducted at cold-start conditions, a particularly damaging time for engines, tests were designed to protect the engine. Prior to testing, the engine was motored at $1500 \mathrm{rpm}$ for five minutes to ensure adequate lubrication before firing. Tests were then conducted at a speed of $1500 \mathrm{rpm}$ and $50 \%$ load, which at these conditions corresponds to $14 \mathrm{Nm}$ of torque. These conditions are typical of a vehicle operating at low speed and with gentle load applied, as is typically recommended for engines in the warm-up period. Fuel and air flow were measured independently and adjusted to run stoichiometric conditions. This calculation was further validated by a lambda sensor mounted in the exhaust downpipe. Further details on the engine test conditions are detailed in Table 2-3.

\begin{tabular}{cc}
\hline Parameter & Condition \\
\hline Test Duration & $4 \mathrm{Hrs}$ \\
\hline Fuel & Standard $(\mathrm{Ab})$ or HBP $(\mathrm{Ba})$ \\
\hline Lubricant & Shell $5 \mathrm{~W}-30$ \\
\hline Engine Compression & $9: 1$ \\
\hline Ignition Control & $11^{\circ} \mathrm{BTDC}$ \\
\hline $\begin{array}{c}\text { Initial Sump } \\
\text { Temperature }\end{array}$ & $21^{\circ} \mathrm{C}$ \\
\hline Air Temperature & $30^{\circ} \mathrm{C}$ \\
\hline $\begin{array}{c}\text { Coolant Cut-in } \\
\text { Temperature }\end{array}$ & $90^{\circ} \mathrm{C}$ \\
\hline Engine Speed & $1500 \mathrm{rpm}$ \\
\hline Engine Load & $50 \%$ of full
\end{tabular}

Table 2-3 Cold-Start Engine Test Parameters

Once firing, sump samples were taken at fifteen minute intervals and TPS sample vials changed once a volume in excess of $3 \mathrm{ml}$ was collected. Once a test had finished samples were kept in air tight containers and refrigerated prior to analyses.

\subsubsection{Steady-State Tests}

These tests were designed to give insight into typical levels of fuel dilution and fuel additive in the cylinder liner oil film at varying engine conditions, and were conducted at steady-state conditions. Before sample vials were fitted to the TPS system, the engine was warmed up and stabilised at conditions detailed in the steadystate test matrix detailed in Table 2-4 and Table 2-5. 


\begin{tabular}{cc}
\hline Parameter & Condition \\
\hline Test Duration & 3 Hrs \\
\hline Fuel & Standard (Ab) \\
\hline Lubricant & Shell $5 \mathrm{~W}-30$ \\
\hline Engine Compression & $9: 1$ \\
\hline ggnition Control & $11^{\circ} \mathrm{BTDC}$ \\
\hline Sump Temperature & $90^{\circ} \mathrm{C}$ \\
\hline Air Temperature & $30^{\circ} \mathrm{C}$ \\
\hline Coolant Cut-in & $90^{\circ} \mathrm{C}$ \\
\hline Temperature & $2000 / 2500 / 3000 \mathrm{rpm}$ \\
\hline Engine Speed & $50 / 100 \%$ of full \\
\hline Engine Load
\end{tabular}

Table 2-4 Steady State Engine Test Parameters

The engine was perceived to be at steady-state conditions once all temperature measurements, taken at various locations around the engine, had reached an equilibrium. Once at steady-state conditions the test duration was 3 hours, and as with the TRZ engine tests a single sample was collected in this time, unless an unusually high flow rate necessitated the use of two sample bottles.

\begin{tabular}{ccc}
\hline & \multicolumn{2}{c}{ Load (\% of full) } \\
\hline $\begin{array}{c}\text { Engine Speed } \\
(\mathrm{rpm})\end{array}$ & 50 & 100 \\
\hline 2000 & $\bullet$ & $\bullet$ \\
\hline 2500 & $\bullet$ & $\bullet$ \\
\hline 3000 & $\bullet$ & $\bullet$ \\
\hline
\end{tabular}

Table 2-5 Steady State Engine Test Matrix

\subsection{Post Test Lubricant Analysis}

Prior to analysis, sample vials were placed in air tight bags and refrigerated. It was known that only small quantities of sampled lubricant were available for testing in most cases, thus limiting the amount of analyses which could be conducted. In order to satisfy the primary objectives of the test series it was necessary to analyse the lubricant for both fuel and potassium marker concentration. This analysis was conducted at the Shell Global Solutions (UK) analytical laboratory in the Thornton Research Centre. Where remaining sample volume permitted, dynamic viscosity testing was conducted on the lubricant samples at the University of Leeds by the author. 


\subsubsection{Fuel Dilution Measurement by Gas Chromatography}

Gas Chromatography (GC) was used to determine the amount of fuel dilution in the lubricant samples. The process essentially involves adding a marker to the lubricant sample prior to analysis, which takes place in a metal coated column with a flame ionisation detector. Essentially, as the response factors for the marker and the 'lights' (i.e. the fuel) are the same, the marker serves as an internal calibrant. The repeatability of the fuel dilution measurement process is thought to be very high and is suggested to be in the region of $\pm 0.3 \mathrm{wt} \%$ (Shell Global Solutions (UK)). As a result of the small error associated with the determination of fuel dilution in a sample, error bars have not been included on presented results.

\subsubsection{Potassium Concentration Testing by ICP-AES Analysis}

Inductively Coupled Plasma - Atomic Emission Spectrometry (ICP-AES) was used to determine the concentration of Potassium in the lubricant samples. Essentially this is a process where the lubricant is diluted with some solvent in order to make aspiration easier and then analysed by Inductively Coupled Plasma-Mass Spectrometry (ICP-MS) using a calibration made from standards of known potassium concentration. The error associated with the potassium concentration measurement technique is thought to be $\pm 10 \%$ (Shell Global Solutions (UK)).

\subsubsection{Dynamic Viscosity Measurement}

A Bohlin CV120HR rheometer was used to measure the dynamic viscosities of both the sump and cylinder liner lubricants (Figure 2-7). Measurements were not conducted on samples taken in the top ring zone sampling tests due to the lack of remaining sample after fuel dilution and potassium concentration analysis, and as such measurements were only conducted on cylinder liner samples.

The machine was fitted with a $40 \mathrm{~mm}$ diameter plate-to-plate setup and was controlled at a constant $40^{\circ} \mathrm{C}\left( \pm 0.3^{\circ} \mathrm{C}\right)$. This temperature was selected as it was known that samples containing a significant proportion of volatiles such as fuel are dangerous to measure at elevated temperatures. In addition, their evaporation makes achieving stable test conditions difficult. A controlled volume of sample lubricant was added to the base plate, and the top plate brought down to a gap of 100 microns. The top plate was then rotated at high speed until an average shear rate of $8.81 \mathrm{e}+3$ $(1 / s)$ was achieved. Once at the maximum shear rate the dynamic viscosity of the lubricant was measured and recorded. The total measurement process took 3 minutes to complete. Once the test was completed the spindle was stopped and raised before cleaning both plate surfaces thoroughly with isopropanol. 


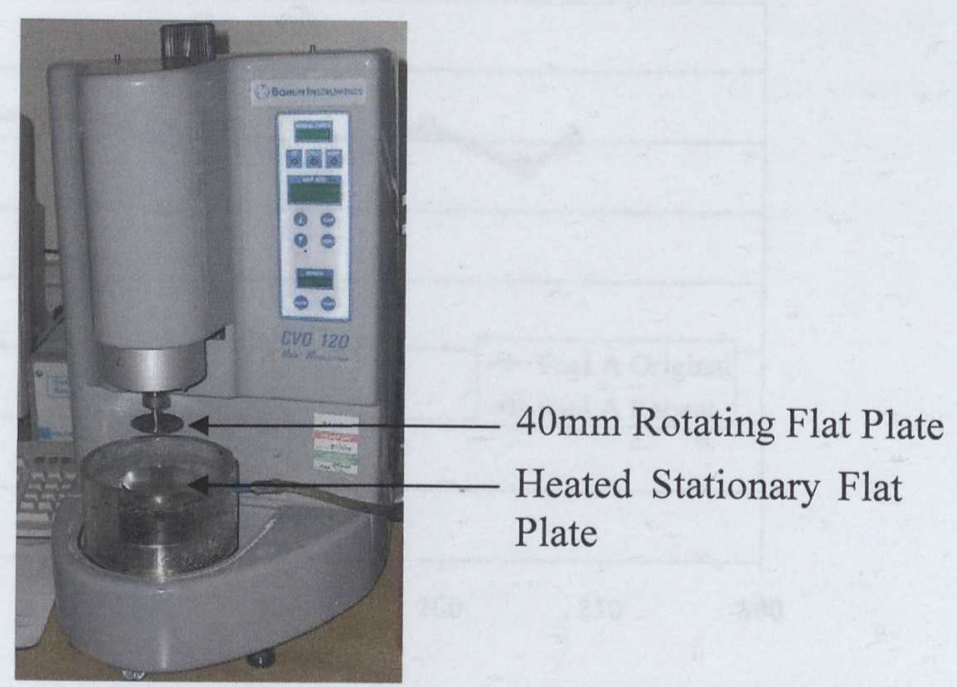

Figure 2-7 Bohlin CV120HR Dynamic Viscometer

A method was designed to measure the repeatability of the dynamic viscosity measurements taken using the Bohlin CV120 HR rheometer. This method was restricted by the amount of sample remaining after the fuel dilution and potassium concentration analysis. A separate method was used for investigating the error associated with repeatability for sump samples and the samples taken from the TPS system as these samples were much more volumous and permitted more complete analysis. In addition, on inspection of the samples taken from the TPS system it was clear that repeatability would need to be calculated for each sampling location in order to give an accurate representation of the error associated with the viscosity measurement methodology.

\subsubsection{Sump Sample Viscosity Measurement Repeatability}

All samples were tested on the same day using the methodology described above, and the exact same test repeated one week later, and again the following week. The percentage deviation from the average was then calculated between the three series of values and was shown to be $1.95 \%$. However, it is acknowledged by the author that it is not ideal to state error based on only three repeated tests, and that more are required to make the value truly statistically significant. Given the lack of sample volume available it is thought the value is as accurate as can be for this particular situation and aids analysis of the data. An example repeatability data set is detailed in Figure 2-8. 


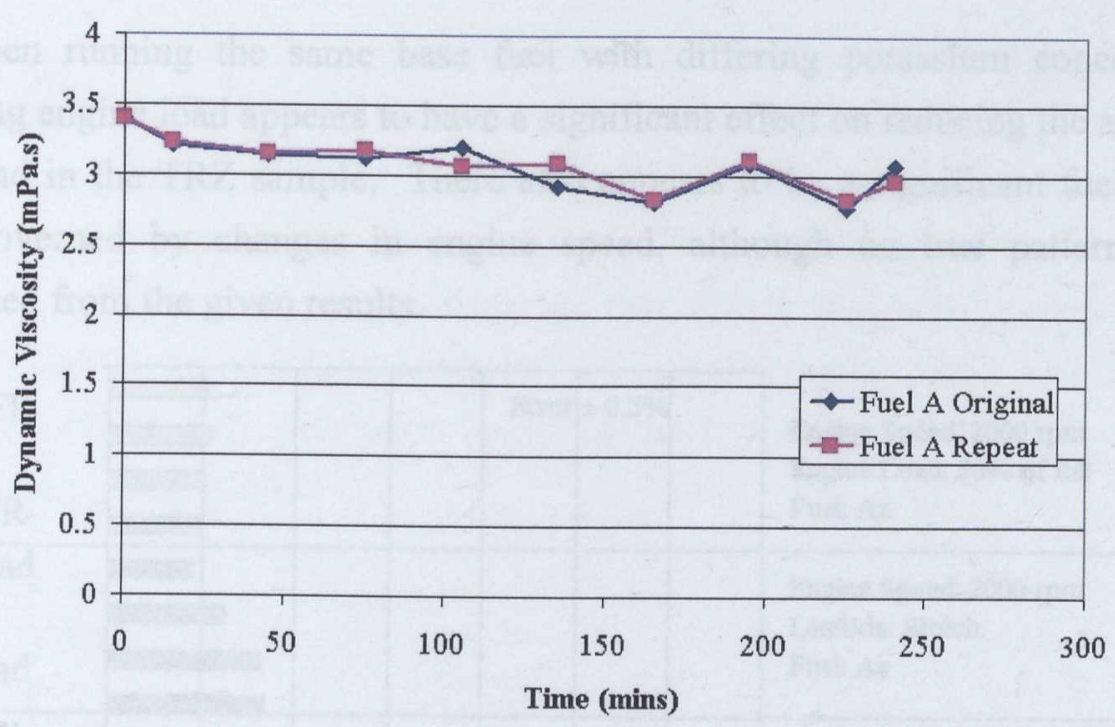

Figure 2-8 Sump Sample Viscosity Analysis Repeatability

\subsubsection{Three Point Sample Viscosity Measurement Repeatability}

Due to the limited sample volume remaining from the portfolio of previous tests, the repeatability analysis tests conducted to give an idea of error for the viscosity measurement methodology was restricted. Tests were conducted on samples which had enough sample volume remaining to give meaningful data. Samples were all tested on the same day, and repeat testing conducted one week later, and again the following week. Similarly to the calculations performed with the sump samples, a value was obtained indicating the percentage deviation from the average for each sampling location, Table 2-6.

\begin{tabular}{cccc}
\hline Fuel & Bottom $(\mathrm{Av} \%$ dev $)$ & Middle $(\mathrm{Av} \%$ dev $)$ & Top (Av \% dev) \\
\hline Fuel $\mathrm{Ab}$ & 5.57 & 1.18 & 1.27 \\
\hline
\end{tabular}

Table 2-6 Calculated Error Associated with TPS Viscosity Measurement

\subsection{Top Ring Zone Sampling Results and Analysis}

On inspection of Figure 2-9 it is clear that when running on fuel $\mathrm{Ab}$, a standard fuel not dissimilar to that which can be purchased at the pump, the top ring zone lubricant typically contains between 5 and $12 \mathrm{wt} \%$ fuel dilution. Running the engine on fuel $\mathrm{Ba}$ (high boiling point fuel) has a significant effect on the amount of fuel dilution found in the top ring zone. The result associated with the high boiling point fuel shows $33 \mathrm{wt} \%$ fuel dilution is found in the top ring zone lubricant. The air / fuel ratio condition appears to have little effect on fuel dilution in the sample, with values of approximately $5 \mathrm{wt} \%$ found at all mixture conditions. Little effect is also 
seen when running the same base fuel with differing potassium concentration. Increasing engine load appears to have a significant effect on reducing the amount of fuel found in the TRZ sample. There also appears to be a significant fuel dilution effect governed by changes in engine speed, although no true pattern can be determined from the given results.

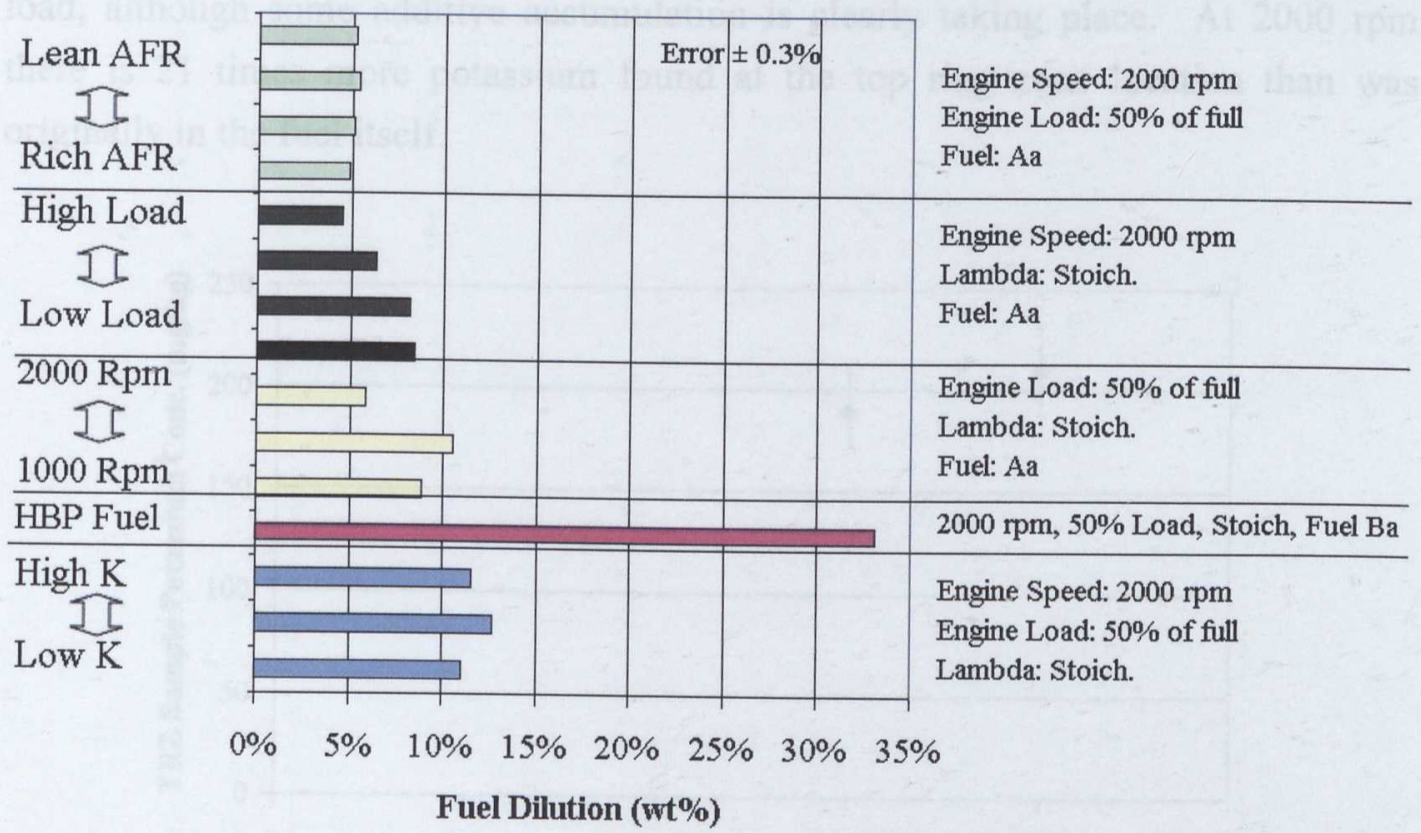

Figure 2-9 Top Ring Zone Fuel Dilution Variation with Engine Condition

Figure 2-10 shows that increasing engine load appears to have an almost exponential affect on the detectable amount of potassium found in the TRZ sample. Given that the fuel used in this test was $\mathrm{Aa}$, a standard fuel containing just $10 \mathrm{mg} / \mathrm{kg}$ potassium, the graph depicts an accumulation of $770 \mathrm{mg} / \mathrm{kg}$ at full load indicating there is 77 times more additive in the lubricant sample than in the initial fuel.

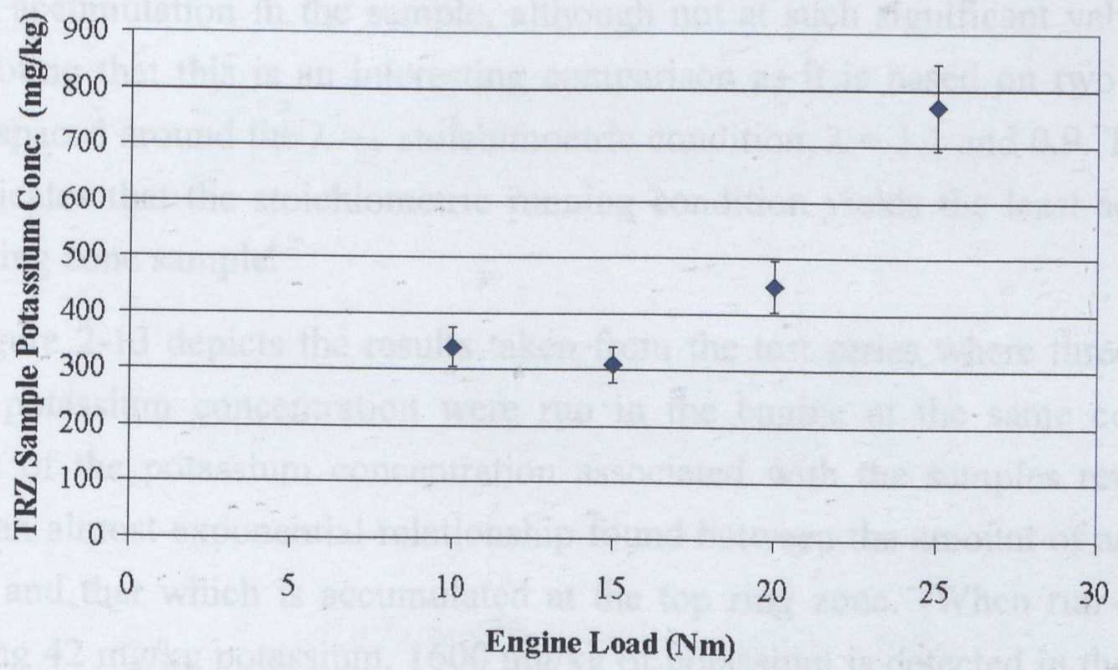

Figure 2-10 Variation of Potassium Concentration with Varying Engine Load 
Figure 2-11 shows the potassium concentration results as a function of varying engine speed, and suggests an increase in detectable potassium in the TRZ sample with increasing speed, although this is not thought to be significant as values fall within those encompassed by errors associated with the analytical technique. The effect does not appear to as significant as that witnessed when increasing engine load, although some additive accumulation is clearly taking place. At $2000 \mathrm{rpm}$ there is 21 times more potassium found at the top ring zone location than was originally in the fuel itself.

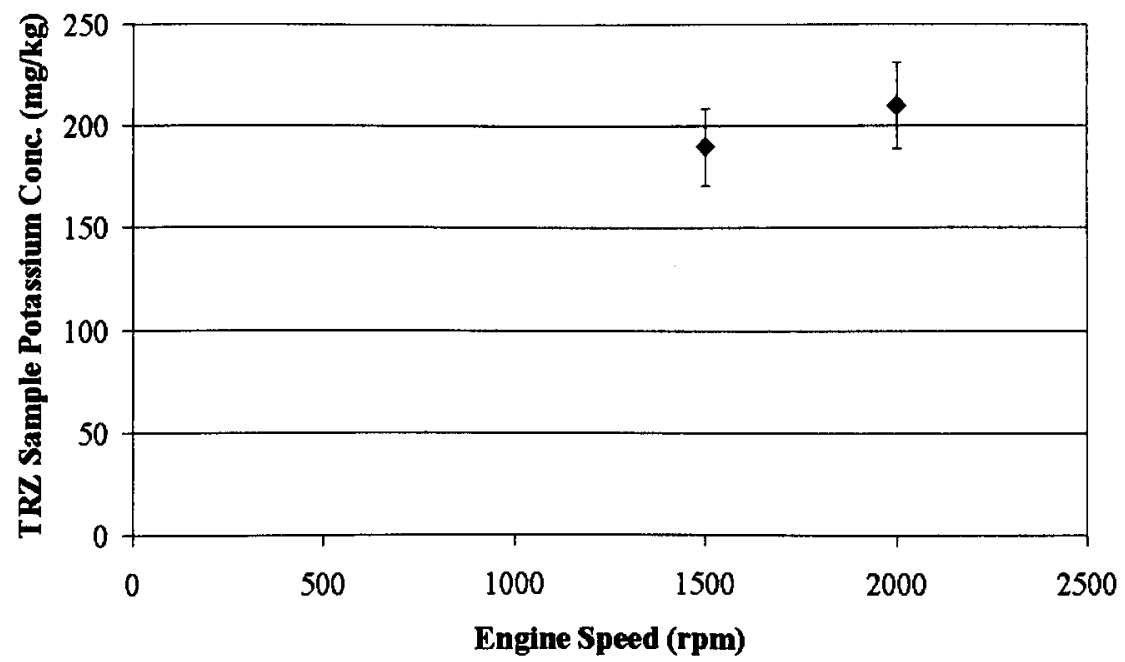

Figure 2-11 Variation of Potassium Concentration with Varying Engine Speed

Figure 2-12 shows the potassium concentration results as a function of varying lambda ratio, and suggests running the engine lean permits greater additive accumulation at the top ring zone. In this case the accumulation is 53 times greater than that supplied by the fuel. The test run at rich conditions also indicates increased additive accumulation in the sample, although not at such significant values. It is worth noting that this is an interesting comparison as it is based on two data sets equally spaced around the $\lambda=1$ stoichimoetric condition, $\lambda=1.1$ and 0.9 . The figure also indicates that the stoichiometric running condition yields the least additive in the top ring zone sample.

Figure 2-13 depicts the results taken from the test series where three fuels of varying potassium concentration were run in the engine at the same conditions. Analysis of the potassium concentration associated with the samples reveals that there is an almost exponential relationship found between the amount of additive in the fuel and that which is accumulated at the top ring zone. When run on a fuel containing $42 \mathrm{mg} / \mathrm{kg}$ potassium, $1600 \mathrm{mg} / \mathrm{kg}$ of potassium is detected in the top ring zone sample indicating an accumulation of 38 times. 


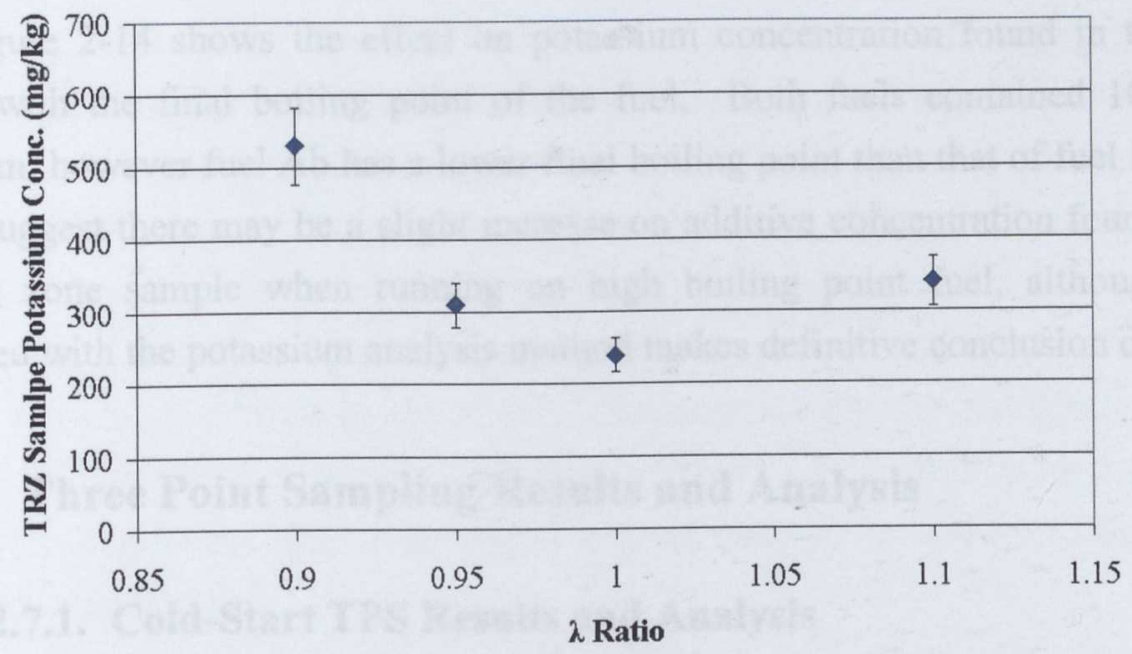

Figure 2-12 Variation of Potassium Concentration with Varying Lambda Ratio

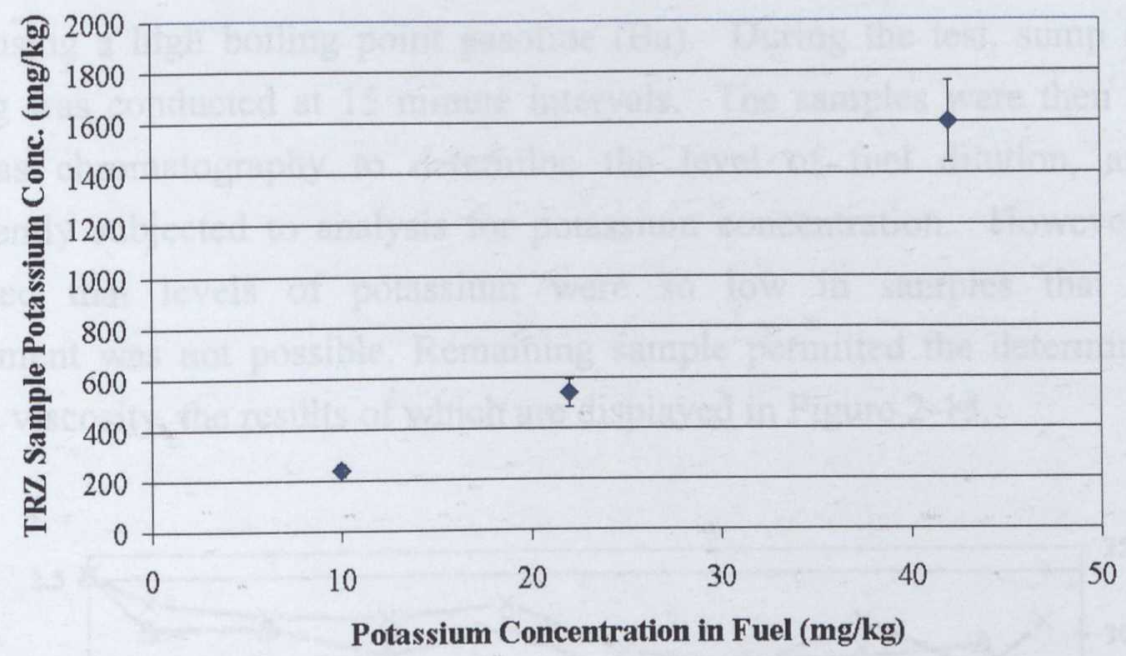

Figure 2-13 Variation of TRZ Potassium Concentration with Varying Fuel Potassium Concentration

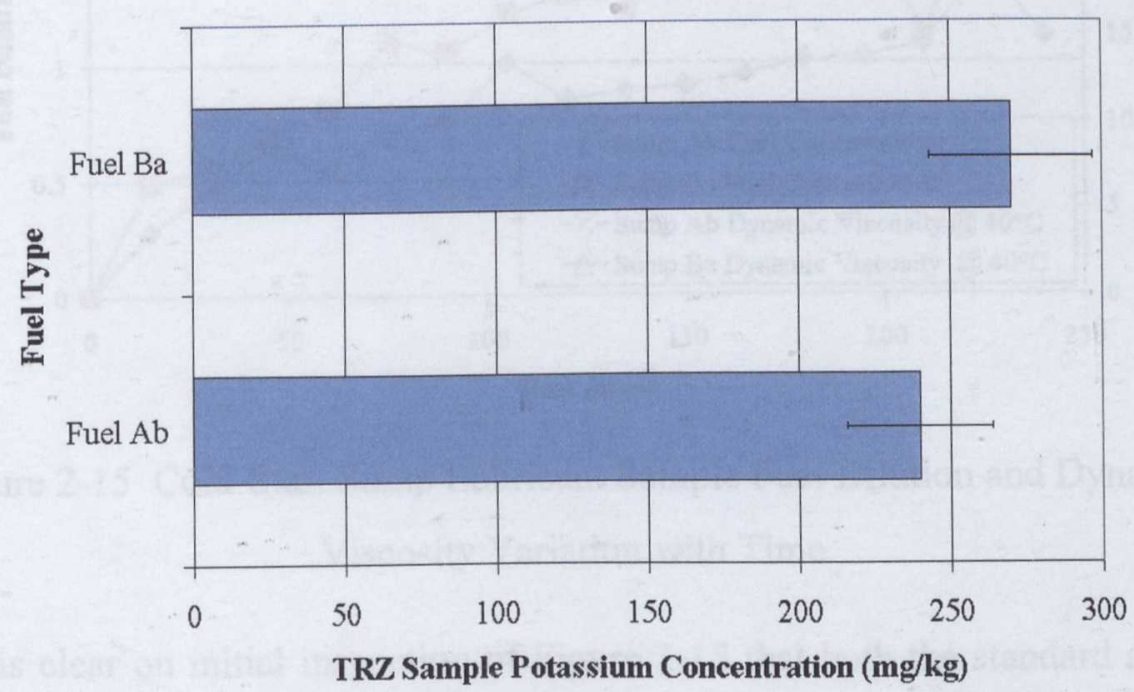

Figure 2-14 Variation of Potassium Concentration with Different Boiling Point Fuels 
Figure 2-14 shows the effect on potassium concentration found in the TRZ sample with the final boiling point of the fuel. Both fuels contained $10 \mathrm{mg} / \mathrm{kg}$ potassium, however fuel $\mathrm{Ab}$ has a lower final boiling point than that of fuel $\mathrm{Ba}$. The results suggest there may be a slight increase on additive concentration found in the top ring zone sample when running on high boiling point fuel, although error associated with the potassium analysis method makes definitive conclusion difficult.

\subsection{Three Point Sampling Results and Analysis}

\subsubsection{Cold-Start TPS Results and Analysis}

Two tests were conducted where the engine was run from cold-start conditions for four hours. The first test was conducted using a standard gasoline $(\mathrm{Ab})$ and the second using a high boiling point gasoline $(\mathrm{Ba})$. During the test, sump lubricant sampling was conducted at 15 minute intervals. The samples were then analysed using gas chromatography to determine the level of fuel dilution, and were subsequently subjected to analysis for potassium concentration. However, it was discovered that levels of potassium were so low in samples that accurate measurement was not possible. Remaining sample permitted the determination of dynamic viscosity, the results of which are displayed in Figure 2-15.

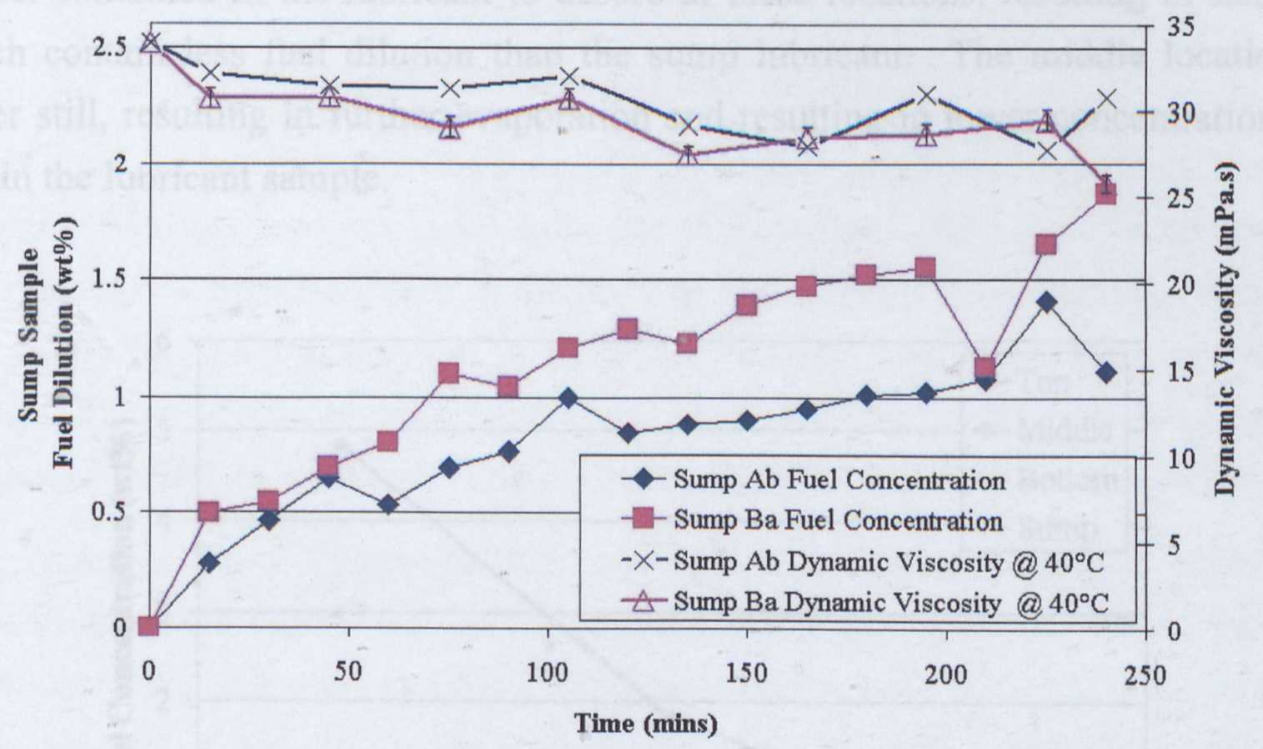

Figure 2-15 Cold-Start Sump Lubricant Sample Fuel Dilution and Dynamic Viscosity Variation with Time

It is clear on initial inspection of Figure 2-15 that both the standard and High Boiling Point (HBP) fuel are shown to accumulate in the engine sump at levels detectable in just fifteen minutes, and that the level of dilution is more severe when 
running on HBP fuel. The fuel appears to accumulate in the sump in an approximately linear trend with respect to time throughout duration of the four hour test period. It is interesting to note that the system does not reach an equilibrium dilution level within the test duration, and that at the end of the four hour test period the level of fuel dilution was $1.1 \mathrm{wt} \%$ for the standard fuel and $1.9 \mathrm{wt} \%$ for the high boiling point fuel. The dynamic viscosity measurements conducted on the sump samples are also displayed in the figure. Not surprisingly trend suggests that the viscosity of the lubricant sample falls with increasing fuel dilution, and indicates running the engine on $\mathrm{HBP}$ fuel lowers the viscosity of the oil more than when running on standard fuel.

Figure 2-16 and Figure 2-17 represent the fuel dilution measured in the samples taken at three locations on the cylinder liner for the cold-start test conducted with standard fuel and high boiling point fuel respectively. It is clear on inspection of Figure 2-16 that at the start of the test the fuel dilution at the top of the cylinder liner is high, but falls thereafter. Samples collected from the middle and bottom locations exhibit low levels of fuel dilution throughout the test, with the bottom location being more fuel diluted than the middle, and the sump having even more dilution for most of the test. It is hypothesised that the slightly higher temperature of the cylinder liner with respect to the sump is responsible for this phenomenon. The relatively high temperature of the liner at the bottom location causes a small fraction of fuel contained in the lubricant to desorb at these locations, resulting in samples which contain less fuel dilution than the sump lubricant. The middle location is hotter still, resulting in further evaporation and resulting in lower concentrations of fuel in the lubricant sample.

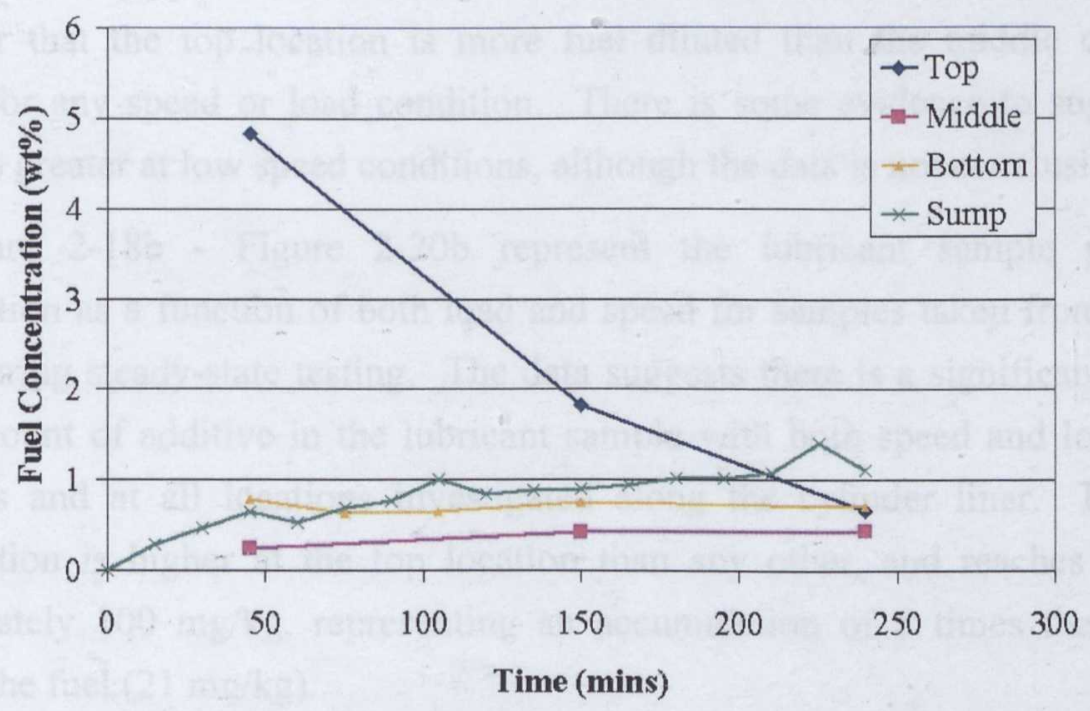

Figure 2-16 Fuel Dilution in Both TPS and Sump Samples for Fuel Ab 
Figure 2-17 is for the test conducted on HBP fuel and clearly shows that fuel dilution at the top of the cylinder liner is high, and rises throughout the test to a maximum of $11 \mathrm{wt} \%$. Trends associated with the middle and bottom samples are similar to those seen when running on standard fuel, although the final samples suggest the sample taken from the middle location is the least fuel diluted, followed by the bottom location, then the sump, then the top location.

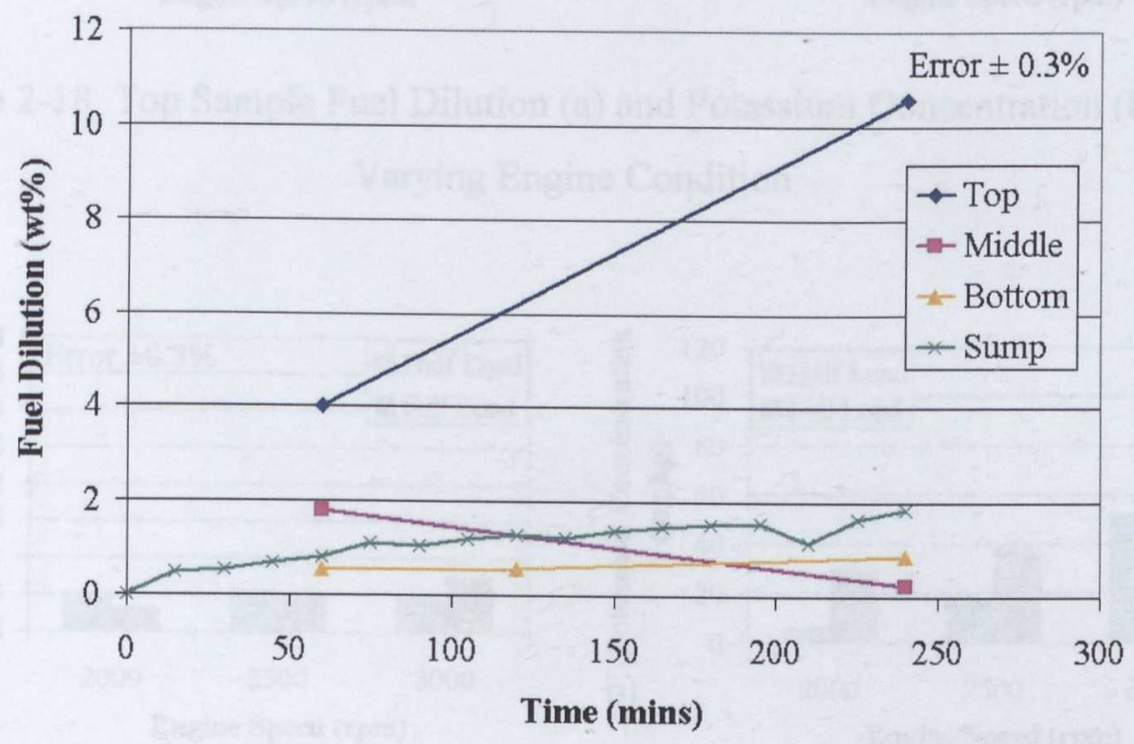

Figure 2-17 Fuel Dilution in Both TPS and Sump Samples for Fuel Ba

\subsubsection{Steady-State TPS Results and Analysis}

Figure 2-18a - Figure 2-20a present fuel dilution as a function of both load and speed for lubricant samples taken from the TPS system during steady-state testing. There are no discernable trends in the level of fuel dilution in the samples, although it is clear that the top location is more fuel diluted than the middle or bottom location for any speed or load condition. There is some evidence to suggest fuel dilution is greater at low speed conditions, although the data is not conclusive.

Figure 2-18b - Figure 2-20b represent the lubricant sample potassium concentration as a function of both load and speed for samples taken from the TPS system during steady-state testing. The data suggests there is a significant increase in the amount of additive in the lubricant sample with both speed and load for all conditions and at all locations investigated along the cylinder liner. Potassium concentration is higher at the top location than any other, and reaches levels of approximately $100 \mathrm{mg} / \mathrm{kg}$, representing an accumulation of 5 times that initially found in the fuel $(21 \mathrm{mg} / \mathrm{kg})$. 

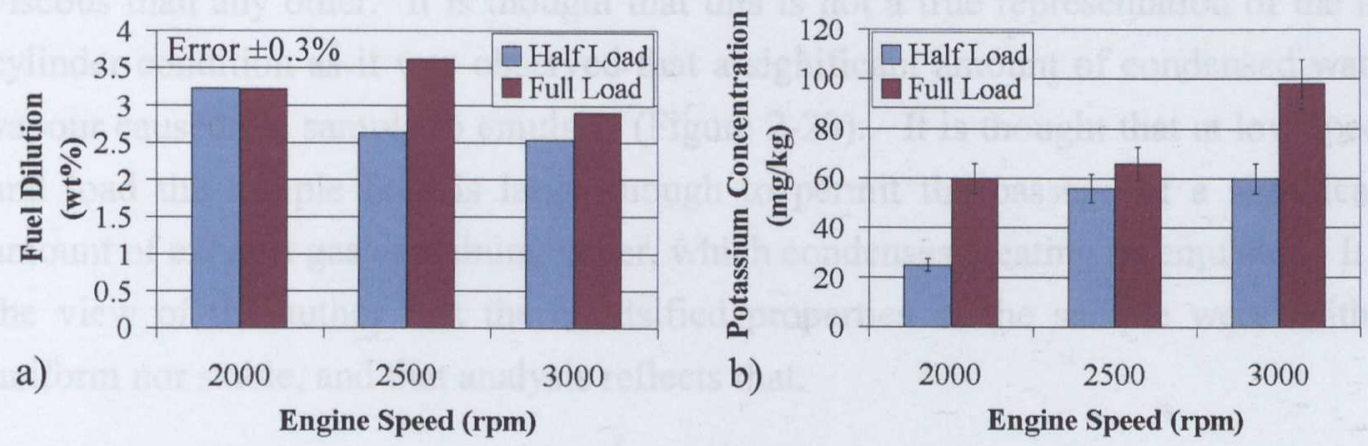

Figure 2-18 Top Sample Fuel Dilution (a) and Potassium Concentration (b) with Varying Engine Condition
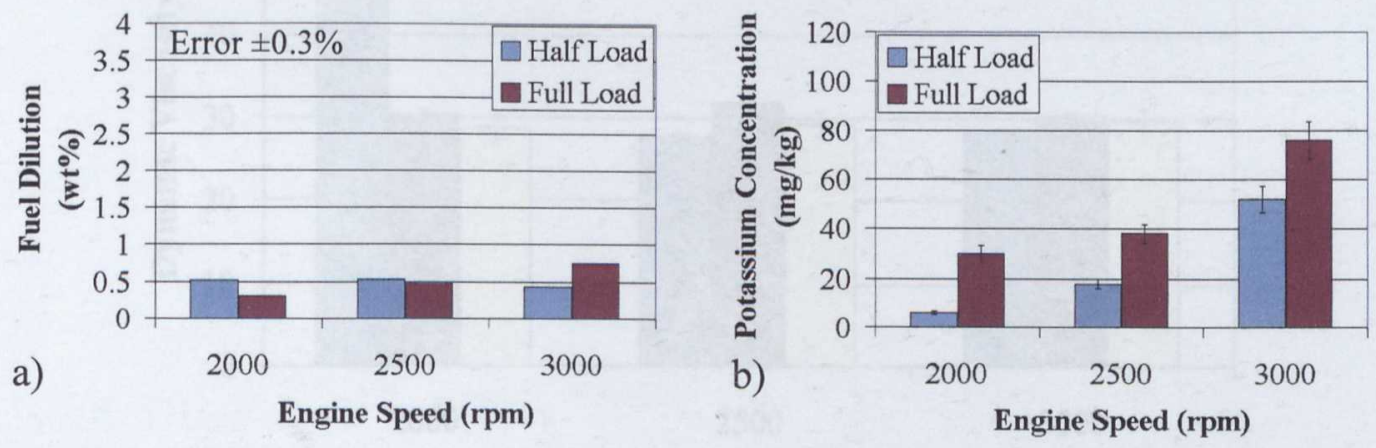

Figure 2-19 Middle Sample Fuel Dilution (a) and Potassium Concentration (b) with Varying Engine Condition
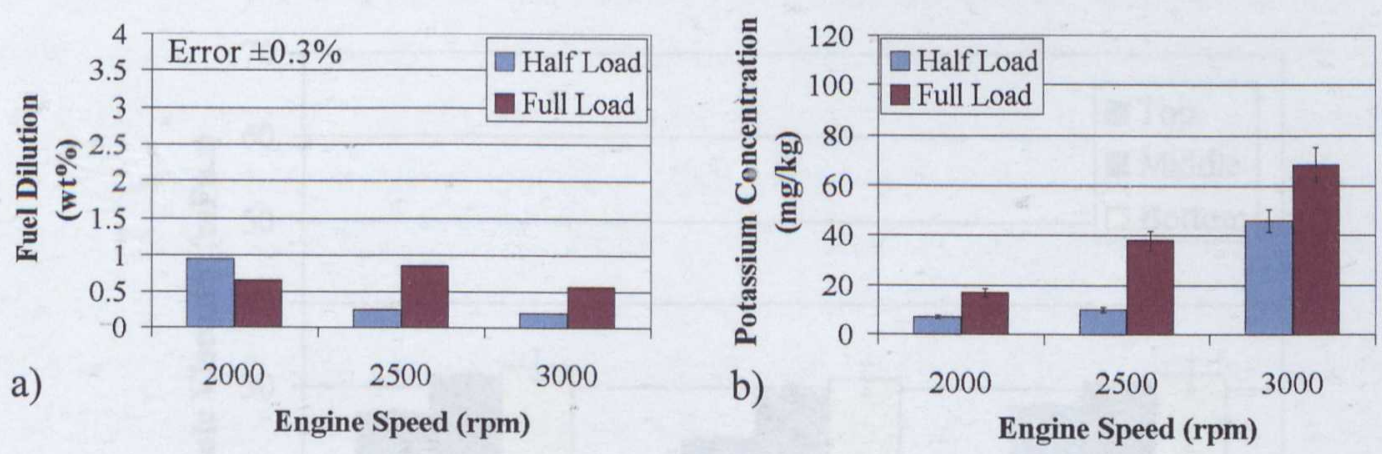

Figure 2-20 Bottom Sample Fuel Dilution (a) and Potassium Concentration (b) with Varying Engine Condition

Figure 2-21 and Figure 2-22 depict the dynamic viscosity variation with engine speed and sample location for both the half and full load engine conditions respectively. Initial inspection of Figure 2-21 depicting the half load condition at $2000 \mathrm{rpm}$ suggests the sample taken from the top of the cylinder liner is much more 
viscous than any other. It is thought that this is not a true representation of the incylinder condition as it was observed that a significant amount of condensed water vapour caused the sample to emulsify (Figure 2-23). It is thought that at low speed and load the sample hole is large enough to permit the passage of a significant amount of exhaust gas containing water, which condenses creating an emulsion. It is the view of the author that the emulsified properties of the sample were neither uniform nor stable, and that analysis reflects that.

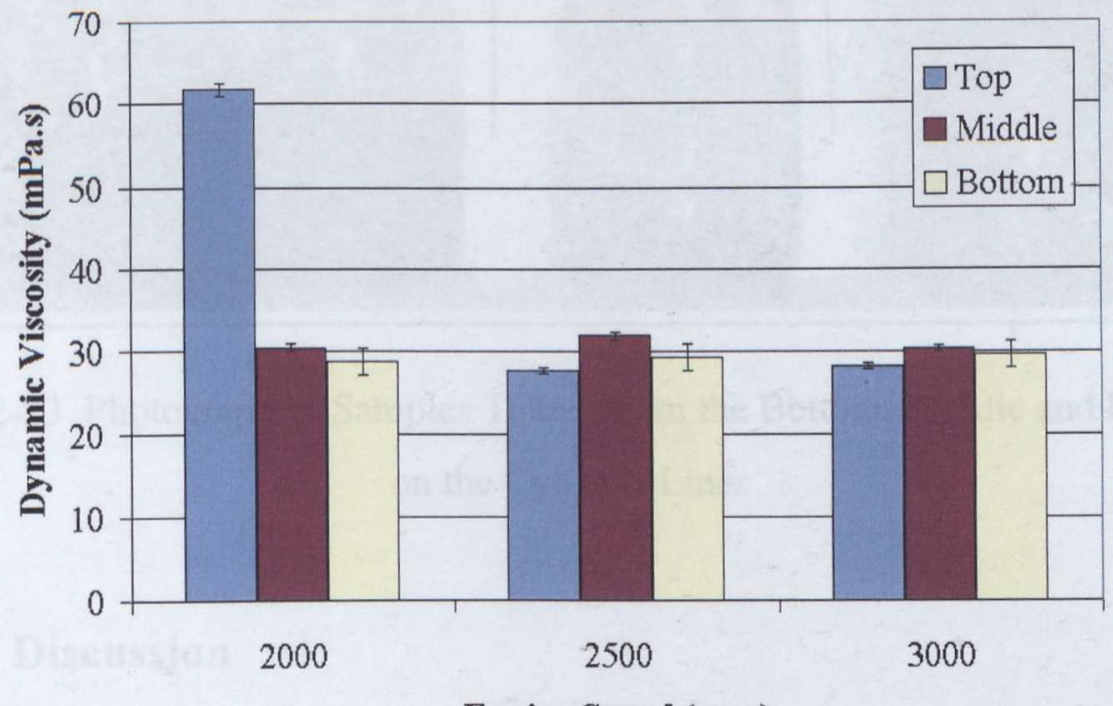

Engine Speed (rpm)

Figure 2-21 Dynamic Viscosity Variation with Engine Speed and Sample Location for Half Load Condition

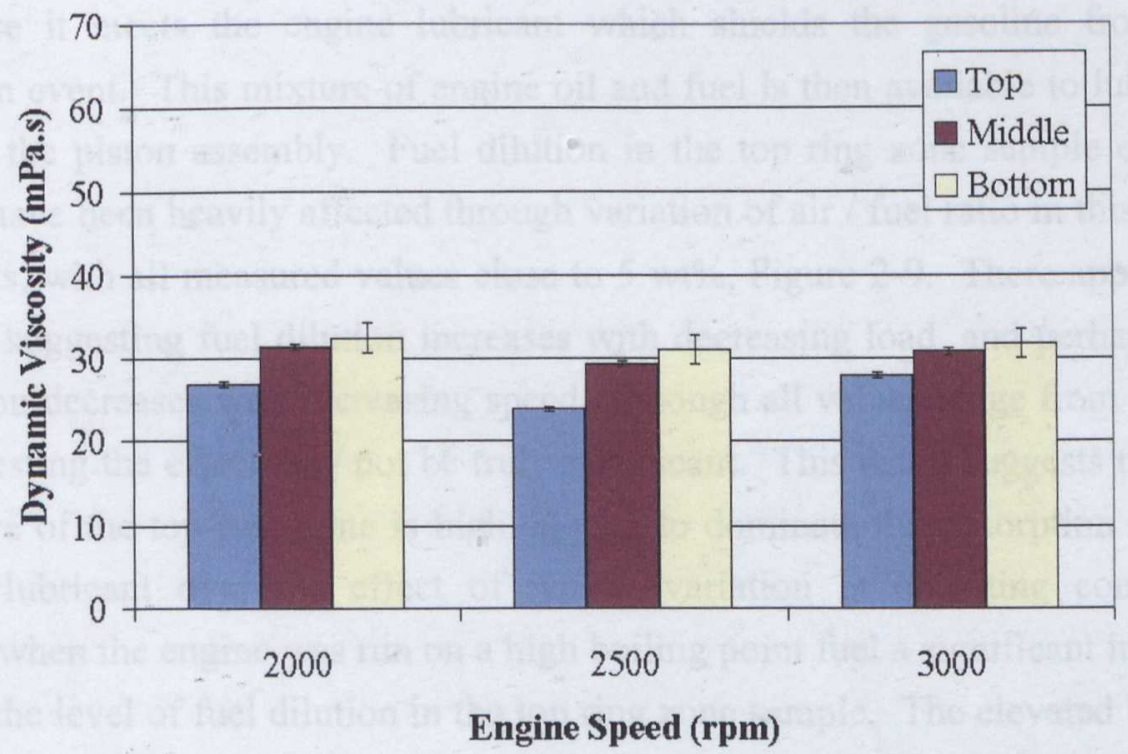

Figure 2-22 Dynamic Viscosity Variation with Engine Speed and Sample Location for Full Load Condition 
In Figure 2-22 there appears to be little to differentiate the samples from one another when comparing measured dynamic viscosities, although it is thought that the trend of decreased viscosity with position up the liner is significant.

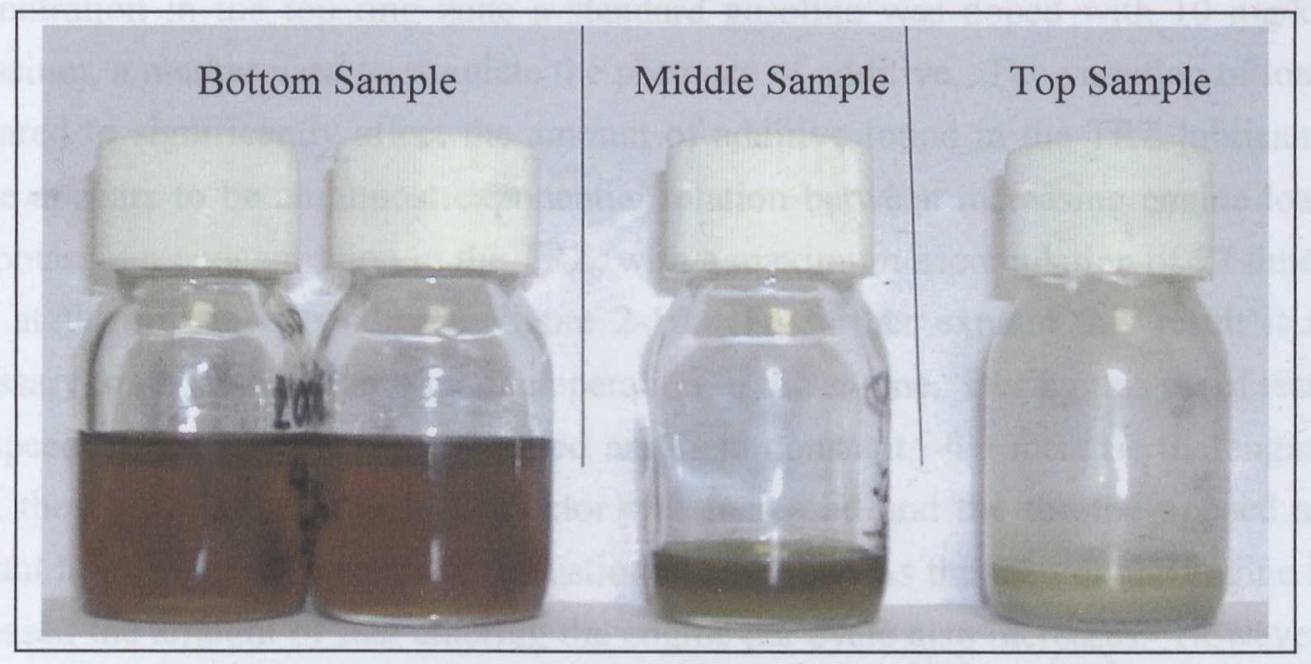

Figure 2-23 Photograph of Samples Taken From the Bottom, Middle and Locations on the Cylinder Liner

\subsection{Discussion}

\subsubsection{TRZ Sampling Discussion}

Gasoline was found in all top ring zone samples taken throughout the investigation. It is suggested that unburnt fuel enters the crevice behind the top ring zone where it meets the engine lubricant which shields the gasoline from the combustion event. This mixture of engine oil and fuel is then available to lubricate the top of the piston assembly. Fuel dilution in the top ring zone sample did not appear to have been heavily affected through variation of air / fuel ratio in this set of engine tests, with all measured values close to $5 \mathrm{wt} \%$, Figure 2-9. There appears to be a trend suggesting fuel dilution increases with decreasing load, and perhaps that fuel dilution decreases with increasing speed, although all values range from 5 - 10 $w t \%$ suggesting the effect may not be truly significant. This result suggests that the temperature of the top ring zone is high enough to dominate the desorption of fuel from the lubricant over the effect of typical variation in operating condition. However, when the engine was run on a high boiling point fuel a significant increase is seen in the level of fuel dilution in the top ring zone sample. The elevated boiling point of the fuel with respect to a standard fuel means larger quantities of it survive the combustion event. Tests therefore indicate the ability to control the fuel dilution 
in the TRZ lubricant through variation in the boiling point of the fuel, or the temperature of the lubricant with which it interacts.

In order to investigate the effects of varying engine condition on additive concentration in the top ring zone a standard gasoline was doped with $10 \mathrm{mg} / \mathrm{kg}$ potassium, a marker used to simulate the presence of additive. The variation of load appeared to significantly affect the amount of additive found in the TRZ lubricant. There appears to be an almost exponential relation between increasing engine load and potassium concentration in the TRZ, with a maximum accumulation of 77 times seen at the full load condition, Figure 2-10. To further explain this result it is necessary to understand the principle operation of the engine. During this set of tests the speed of the engine was controlled and held constant. To increase the engine load, the bandwidth of the fuel injector was increased and the throttle opened an amount to allow stoichiometric combustion conditions. As the load on the engine is increased the amount of fuel entering the engine per cycle also increases. However, we know that for this condition there are not elevated levels of fuel dilution found in the top ring zone lubricant. It is therefore suggested that the fuel carries the additive to the crevice behind the piston ring during the intake stroke of the engine where it meets a quantity of oil into which it adsorbs. During and after the combustion event, the fuel subsequently desorbs from the film leaving behind the non volatile additive. Repetition of this process facilitates the accumulation of additive in the TRZ lubricant. The mass accumulation of additive in the TRZ lubricant suggests that the crevice behind the top compression ring, the location where the lubricant is sampled, acts like a reservoir where lubricant residence times are sufficient enough to allow the accumulation of additive.

Increasing engine speed was shown to have some effect on the accumulation of additive in the top ring zone sample, although not in such significant magnitude as increasing load. As such there is no conclusive evidence to suggest engine speed has a real affect on additive concentration at the top ring zone.

Varying the air/fuel ratio has an interesting affect on the concentration of potassium measured in the top ring zone sample. Logic suggests that running the engine at a rich condition would result in greater levels of additive accumulation in the sample purely as a result of the increase of additive entering the system per cycle, results show this is the case. However, it is interesting to note that running the engine at lean conditions also increases the amount of additive found in the TRZ sample. It is thought that the explanation of this phenomenon may lie within the specific characteristics of the engine. At stoichiometric conditions, as the term suggests, conditions are optimum for the usage of the fuel, outside these conditions the system may behave uncharacteristically as several parameters governing the 
engines operation become affected. This could possibly result in different mixing conditions in the cylinder effecting fuel / lubricant interaction.

To investigate effects associated with variation of additive treatment level, fuels with varying concentrations of potassium were run in the engine under identical operating conditions. Prior to being tested in the engine, the potassium treatment level in each fuel was measured and shown to be $10 \mathrm{mg} / \mathrm{kg}, 21 \mathrm{mg} / \mathrm{kg}$ and $42 \mathrm{mg} / \mathrm{kg}$. The corresponding levels of potassium concentration found in the top ring zone samples appeared to reflect an approximate exponential trend, where an initial treatment level of $42 \mathrm{mg} / \mathrm{kg}$ results in $1600 \mathrm{mg} / \mathrm{kg}$ potassium at the TRZ, Figure 2-13. This result is not too surprising as it essentially suggests applying more additive to a system results in more accumulation in the top ring zone. This result highlights the need to test fuels with treatment levels over $42 \mathrm{mg} / \mathrm{kg}$ to investigate the optimum treatment level.

Tests conducted with standard and high boiling point fuel reveal that the boiling point of the fuel has little effect on the accumulation of additive at the top ring zone, Figure 2-14. Results suggest the volatility of the fuel is not a significant factor in the administration of additive to the top ring zone, and that far more significant and influential factors are engine load and fuel treatment level.

\subsubsection{Three Point Sampling Discussion}

Analysis of the data taken from the cold-start tests suggests that as soon as the engine is fired, fuel accumulates in the engine sump in reasonably large quantities. It is thought the passage of the fuel from the combustion chamber is facilitated through one of two main mechanisms. The first suggests the fuel is transported to the sump lubricant in vapour phase by the combustion gasses which blow-by the piston rings under firing conditions. The second hypothesises that the fuel absorbs into the cylinder liner lubricant on injection into the cylinder, and is protected from the combustion event by the lubricant. During the subsequent exhaust and intake strokes the local temperatures and pressures promote the desorbtion from the cylinder liner lubricant where it then exits the engine as unburnt hydrocarbon, or remains on the crankcase side of the system manifesting itself as sump fuel dilution. Tests conducted with high boiling point fuel indicate that the fuel dilution in the sump is heavily dependent on the boiling point of the fuel, thus highlighting incylinder temperatures as a critical influence on sump dilution. Dynamic viscosity results taken from the sump lubricant reflect the influence of fuel dilution almost exactly, and enforce the point that fuel dilution significantly effects the sump lubricant viscosity, Figure 2-15. 
All samples taken from the Three Point Sampling showed presence of fuel in the cylinder liner lubricant, and highlight the excessive dilution of the top location, particularly when running on high boiling point fuel. It is interesting to note that the top location fuel dilution falls with time when running on standard gasoline, but continues to rise when running on high boiling point gasoline, suggesting the boiling point of the fuel is again a truly significant factor influencing the lubrication condition of the engine.

Steady-state sampling tests do not show any significant trends in fuel dilution with varying engine condition, although it is interesting to note that the top cylinder liner lubricant is approximately four times more diluted by fuel than any other location on the liner. The reason for this high level of fuel dilution at the top cylinder liner location follows logic, as it is this lubricant which directly interacts with the fuel once the fuel injector opens. Interestingly the middle and bottom liner locations exhibited similar fuel dilutions suggesting the local situation below the top location has stabilised. It is suggested that it is this lubricant which is transported to the sump with little further desorbtion, as the levels of fuel dilution in the engine sump are typically not dissimilar.

Potassium concentrations measured in the steady-state tests revealed some interesting results. It is clear that additive concentration increases with both speed and load. The mechanism for this accumulation is not thought to be dissimilar to that discussed in the top ring zone sampling section where it is hypothesised that most of the fuel carrying the additive marker desorbs from the lubricant film leaving behind the non-volatile additive. However, accumulation levels of just 5 times were seen at the top liner location, as opposed to 77 times measured in the sample taken from the top ring groove, Figure $2-18 \mathrm{~b}$. This result suggests that the lubrication of the cylinder liner is a much more dynamic system than that of the top ring zone, and perhaps highlights two categories of lubricant residence times, one associated directly with the piston assembly lubrication, and one the cylinder liner.

Dynamic viscosity testing of the samples taken from the TPS tests were inconclusive, and highlighted the difficulty of viscosity measurement of samples containing volatile constituents. However, at the full load condition it is seen that the lubricant is less viscous than that at any position lower down the liner. This result is thought to be a direct result of fuel dilution which acts to lower the viscosity of the cylinder liner lubricant at the top location.

Overall the TPS tests highlight the power of the sampling system, demonstrating the ability of the system to give information which permits the understanding of how the piston assembly is lubricated, and more importantly how this lubrication can be controlled. Tests conducted with potassium marker indicate 
the effectiveness of simulating fuel administered additive to the cylinder liner lubricant, and again permits the beginning of an understanding of how the lubrication of the piston assembly system can be influenced through strategic application of an additive package.

\subsection{Summary}

A portfolio of engine tests was conducted whose purpose was to extract lubricant from strategic locations around the piston assembly / cylinder liner interface. Analysis of these lubricant samples gives us information detailing the lubricating conditions at the piston assembly / cylinder liner interface, and provides us with knowledge which can be utilised to both influence and improve the operation of the piston assembly. On consideration of all the analysis and discussion detailed in this chapter, the following summary can be made.

- Top ring zone sampling is an effective method of extracting piston assembly lubricant directly from the back of the top ring grove of a firing engine. A top ring zone sampling system was developed in this work, and as such manifests itself as one of the most advanced systems in existence in terms of both engine speed and durability.

- Lubricant sample extracted from the TRZ was shown to be diluted with significant amounts of fuel at all engine conditions tested. Typical amounts of fuel dilution range from $5-12 \mathrm{wt} \%$. Increasing load appears to increase the fuel dilution within the top ring zone, whereas increasing speed appears to decrease it. However, the final boiling point of the fuel appears to be the most significant influence on the amount of fuel found in the top ring zone and was shown to increase TRZ lubricant fuel dilution to approximately $33 \mathrm{wt} \%$.

- Doping gasoline with a potassium marker is an effective way of simulating the presence of additive, providing a means of quantification of additive concentration in analysed lubricant samples.

- Additive concentration was increased in the top ring zone through increasing engine speed and operating with high boiling point fuel, although most significant influencing factors appear to be engine load and additive treat level. When run at the full load condition, lubricant sampled from the top ring groove contained 77 times more additive than the original treatment level of the fuel, showing significant accumulation occurs. 
- A novel piece of equipment was developed which permitted the sampling of cylinder liner lubricant at three locations simultaneously under fired conditions for a range of speeds and loads. The equipment was shown to be capable of sampling at conditions of $3000 \mathrm{rpm}$ and full load, and it is thought that greater speeds would not cause a problem, although they were not investigated in this work.

- Analysis of sump samples taken in a cold-start test revealed measurable amounts of fuel dilution within 15 minutes. Data collected also reveals that the final boiling point of the fuel is a significant factor influencing the level of fuel dilution in the sump, with high boiling point fuels causing greater dilution.

- Research leads the hypothesis that the transport of fuel to the engine sump lubricant takes place by one of two mechanisms; 1) fuel passes the piston rings in the blow-by gasses in a vapour phase, 2) fuel adsorbs into the cylinder liner lubricant, which is returned to the sump in a liquid phase through the dynamic action of the piston ring / cylinder liner interface.

- Cylinder liner lubricant fuel dilution does not appear to be heavily influenced through changes in engine speed or load. However tests revealed that the lubricant at the top of the cylinder liner has a greater amount of fuel dilution than either the middle of bottom position. Typical measurements suggest 4 times more fuel dilution at the top location than any other.

- A concentration of potassium was measured in the samples taken from the three locations on the cylinder liner wall, and was shown to increase with both engine speed and load. At the $3000 \mathrm{rpm}$ and full load the top location on the cylinder liner wall was shown to contain a concentration of potassium additive 5 times greater than that which was originally in the fuel suggesting some accumulation occurs.

- Results suggest that the lubrication of the cylinder liner is a much more dynamic system than that of the top ring zone, and perhaps serves to highlight two categories of lubricant residence times, one associated directly with lubrication of the piston assembly, and one the cylinder liner.

- The development of novel lubricant sampling systems permits the beginning of an understanding as to how the piston assembly is lubricated in detail, and more importantly how this lubrication can be 
controlled. Strategic test design utilising gasoline doped with potassium marker has indicated the effectiveness of simulating fuel administered additive to the cylinder liner lubricant and strengthens the foundation of understanding underpinning the potential effect of strategic application of gasoline additive package. 


\section{Tribometer Investigation of Environmentally Friendly Friction Modifier Additives}

In this chapter a series of tests which were conducted on a Plint TE-77 High Frequency Reciprocator Tribometer are described. The machine provides an ideal opportunity to simulate the arduous conditions at the top ring zone of a firing engine, the area where boundary friction modifiers are most necessary. However, unlike an investigation into engine sump lubricants containing friction modifier, it is extremely difficult and dangerous to mimic gasoline with friction modifiers using a bench-top tribometer due to the inherent flammability. As a result the development of a standardised test with operating conditions selected to simulate the situation in a repeatable and safe manner is presented.

Additives under investigation all conformed to the CHON philosophy, consisting only of carbon, hydrogen, oxygen and nitrogen. This type of additive is considered more desirable than the organo-metallic lubricant friction modifier additives. Tests were developed to allow the comparison of different friction modifier additives under the same test conditions, allowing the performance of each additive to be screened and suggesting which might be the most beneficial in the engine environment. Through careful interpretation of the data taken from the machine it is possible to characterise an additives performance in terms of the speed of reaction, the extent of friction reduction, the extent of film formation, the tenacity of film formation as well as information about the mechanism which leads to friction reduction. Test results were used to select families, or species, of molecule which would be most effective at friction modification in the engine environment. Through analysis of trends in friction reduction with respect to the chemical family from which the friction modifier additive comes, it is possible to hypothesise chemicals capable of even greater friction reduction. Information gained from the portfolio of tests detailed above allow hypotheses to be formed, pre-empting the creation of test lubricant formulations which utilise synergistic additive effects to create ever more effective friction modifier films. Tests were also conducted to investigate the critical concentration of additive required to induce friction modified contact performance and the effect of changes in piston ring coating and surface roughness on additive behaviour. 


\subsection{The Cameron Plint TE-77 Machine and Test Specimens}

The Cameron Plint TE-77 High Frequency Reciprocating Tribometer was used for tests described in this chapter (Figure 3-1). The TE-77 is a pin-on-plate type reciprocating tribometer with a maximum stroke, load and frequency of $25 \mathrm{~mm}$, $1000 \mathrm{~N}$ and $50 \mathrm{~Hz}$ respectively. The machine is a long-established research and development tool for the evaluation of lubricants, materials, surface treatments and coatings. Although typically used for pin-on-plate tests, the machine can take specimens cut from standard components such as piston rings and cylinder liners, thus preserving surface finish, component conformity, material composition and other tribologically important properties (Figure 3-2).

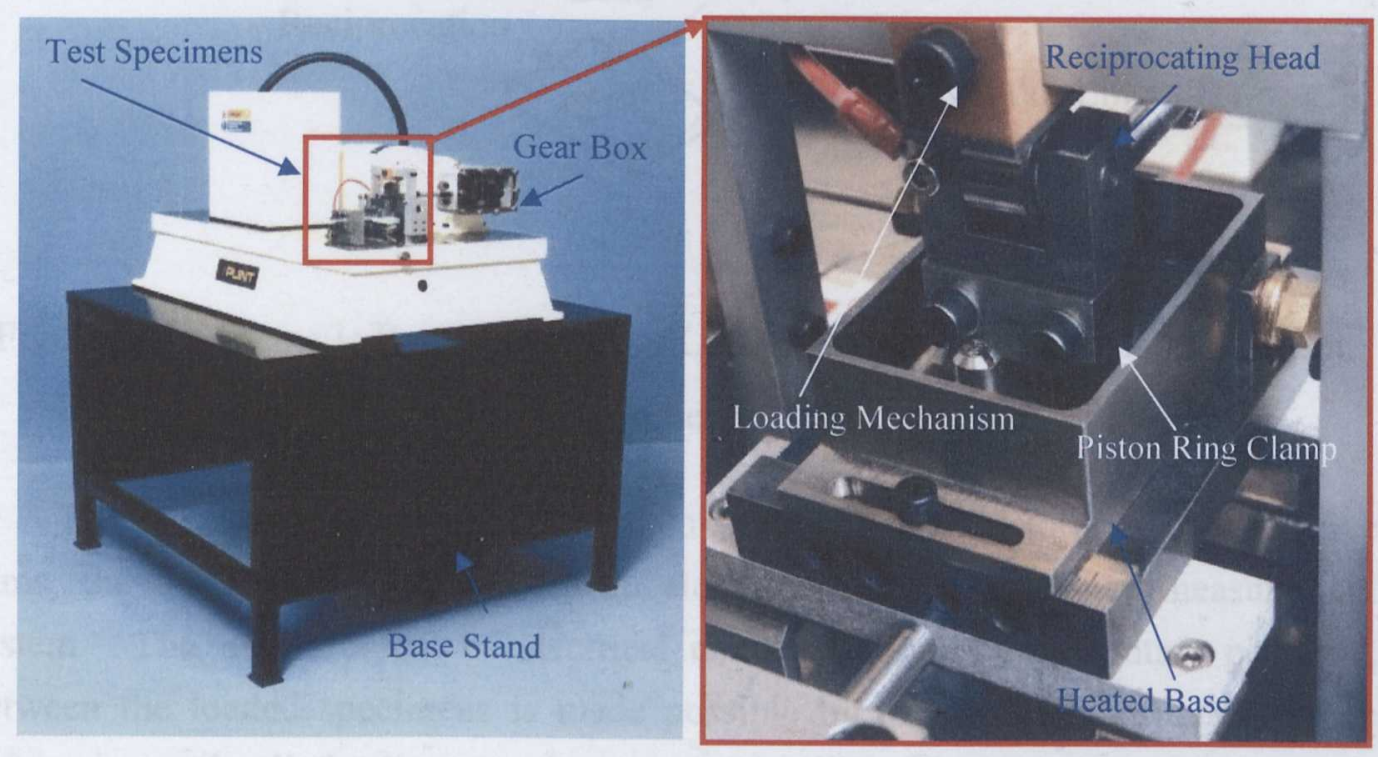

Figure 3-1 Plint TE-77 High Friction Reciprocator Tribometer

The machine features a reciprocating arm into which the piston ring specimen is clamped Figure 3-1. Oscillation of the arm is through a motor driven cam and scotch-yoke mechanism housed in an oil bath filled with gear oil. It is possible to vary the stroke of the arm through manual manipulation of the cam to one of eight positions on the splined drive shaft. The fixed stroke length means gross changes in the friction condition of the contact will not affect the action. Load is applied to the moving specimen through a spring and lever mechanism and is transmitted via a needle roller on the carrier head, which runs against a flat plate creating a constant static load condition, Figure 3-1.

The fixed cylinder liner specimen is mounted in a stainless steel reservoir on a base attachment which conforms to the contour of the back of the liner specimen. The reservoir may be either filled with a known amount of lubricant before a test, or as is employed in this work, fitted with a recirculation system fed from an external 
sump (Figure 3-3, Section 3.3.1). Below the reservoir there is a block of four $200 \mathrm{~W}$ cartridge heaters controlled through a standard feedback heater control unit responding to the temperature measured on the surface of the cylinder liner specimen. The heater block is mounted on flexures which are stiff in the vertical loading direction, but flexible in the horizontal direction. A piezo-electric force transducer connected to the flexures converts the horizontal force into an electrical signal proportional to the force, via a charge amplifier. All of the above is mounted on a common sub-base which provides isolation from vibrations which may occur at high frequency conditions.

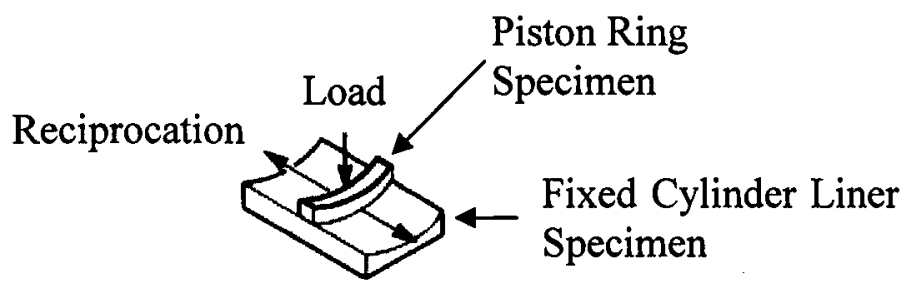

Figure 3-2 Schematic Representation of Loaded Piston Ring Section on Cylinder Liner Specimen in TE-77

To investigate the amount of metal-to-metal contact and formation of additive films, the machine was fitted with an electrical contact resistance measurement system. The measurement of electrical contact resistance, or contact potential, between the loaded specimens is made possible by the electrical isolation of the piston ring and cylinder liner specimens. A 50 millivolt potential is applied across the contact using a Lunn-Furey electrical contact resistance circuit (Furey 1961). In the conventional configuration the voltage signal is taken to a true R.M.S. / D.C. converter amplifier to give a time smoothed average of contact potential, however in this work the raw voltage signal is captured and recorded using a bespoke program discussed in the following section. Variations in the voltage level are indicative of the amount of metallic contact between specimens proving useful when determining the transition of the lubrication regimes in the contact. However, in this work the voltage measurement is also used to give information regarding the formation of non-conducting chemical surface films.

\subsubsection{Data Capture System}

A bespoke data capture system was designed for use in this investigation using a LabVIEW 7.1 software platform. The system comprised two National Instruments 6024E PCI cards synchronised for simultaneous data capture. The first channel 
looked at the amplified voltage output from the force transducer, and captured two full cycles of reciprocation at a sampling rate of $100,000 \mathrm{~Hz}$. Given the normal load applied, and through manipulation of this data, a post processing algorithm calculated the coefficient of friction associated with the contact. Both the coefficient of friction and the raw data from which it was captured were recorded for post test analysis. At the same time, the second channel captured the voltage signal from the contact potential circuit, again at a rate of $100,000 \mathrm{~Hz}$ and for two full reciprocating cycles. This data was logged synchronously against the friction output for post test analysis. The data capture system was fired at user specified intervals, each time creating a file containing the friction and contact potential data with respect to time, as well as plotting the information to the screen for immediate analysis by the user.

\subsubsection{Reciprocator Rig Components}

The specimens used in this work were piston ring and cylinder sections taken from two different engines to provide good conformity between the uncompressed geometry of the piston ring and cylinder liner specimen against which it is loaded. The piston ring selected was a top compression ring made of grey cast iron phosphated with chrome inlay and finished to a barrel face. This is a standard top compression ring taken from a GM (General Motors) 2.0 litre engine piston ringpack. This is the same piston ring type used in the Ricardo Hydra research engine in Chapters 2 and 5. A $25 \mathrm{~mm}$ section of the piston ring was cut $50 \mathrm{~mm}$ from the ringgap, as it was found that the curvature of the ring at this point aided conformity with the cylinder liner specimen under the test conditions employed. In order to achieve good conformity between the non-compressed piston ring and the liner on which it runs, it is not possible to use cylinder liner specimens cut directly from the GM 2.0 litre engine. Good conformity is found between the Jaguar XJ12 cylinder liner and the GM 2.0 litre un-tensioned top compression ring. This liner is also machined from grey cast iron and is finished with a $45^{\circ}-65^{\circ}$ crosshatch plateau hone. The composition of the Jaguar XJ12 cylinder liner is very close to that of the Ricardo Hydra gasoline engine cylinder liner as shown in Table 3-1.

It is usual practice for a new set of specimens to be installed in the machine for each test conducted. However, preliminary investigation revealed that when running tests with piston ring and cylinder liner specimens the initial alignment of the components within the machine is a critical factor determining the contact behaviour and ultimately the friction coefficient. When looking for small differences between the performances of additives, any potential variance of this magnitude was likely to hide all but the most major of effects. For this reason it was decided to use a single set of specimens for a complete test series. Specimens were prepared pre-test by running-in the pair at conditions described in Table 3-2. 


\begin{tabular}{ccc}
\hline Detail & $\begin{array}{c}\text { Hydra Gasoline Engine } \\
\text { Cylinder Liner }\end{array}$ & TE-77 Liner Specimen \\
\hline $\mathrm{C}(\%)$ & $3.0-3.5$ & $3.0-3.5$ \\
\hline $\mathrm{Mn}(\%)$ & $0.6-1.2$ & $0.6-1.0$ \\
\hline $\mathrm{Si}(\%)$ & $1.8-3$ & $1.8-2.5$ \\
\hline $\mathrm{S}(\%)$ & $0.12 \mathrm{max}$ & $0.10 \mathrm{max}$ \\
\hline $\mathrm{P}(\%)$ & $0.4-1.0$ & $0.4-0.6$ \\
\hline Hardness & $230-300 \mathrm{BHN}$ & $230-275 \mathrm{BHN}$ \\
\hline $\mathrm{Ra}(\mu \mathrm{m})$ & $1.57 \mu \mathrm{m}$ (new) & $0.83 \mu \mathrm{m}$ \\
\hline Diameter & $0.38 \mu \mathrm{m}$ (worn) & $90 \mathrm{~mm}$ \\
\hline
\end{tabular}

Table 3-1 Cylinder Liner Specimen Specification

However, using this method does have some disadvantages. Some wear of the specimens undoubtedly occurred throughout the test series, although it was assumed that this influenced the data much less than using new specimens for each test. Cleaning of the specimens was also difficult between tests, as they could not be removed from their respective holders and placed in an ultrasonic bath, as would be usual practice. A cleaning procedure was developed to remedy this and is described in detail in section 3.3.1.

\begin{tabular}{cccccc}
\hline Step & $\begin{array}{c}\text { Duration } \\
(\mathrm{Hrs})\end{array}$ & Speed (rpm) & Load $(\mathrm{N})$ & $\begin{array}{c}\text { Stroke } \\
(\mathrm{mm})\end{array}$ & Temp $\left({ }^{\circ} \mathrm{C}\right)$ \\
\hline 1 & 2 & 2000 & 100 & 4.9 & 80 \\
\hline 2 & 2 & 2000 & 150 & 4.9 & 110 \\
\hline
\end{tabular}

Table 3-2 Running-In Conditions for Piston Ring and Cylinder Liner Specimens

On inspection of the piston ring and cylinder liner specimens after the first tests it was revealed that the components were reasonably well polished. It was decided that no further tests should be conducted on this liner, and subsequently the repeat set of tests were conducted using a separate set of specimens which had been carefully selected, aligned for equally good conformity and run-in. 


\subsection{Organic Friction Modifiers Under Investigation}

It was stated in previous chapters that the use of conventional friction modifiers is being phased out due findings of research which suggests they contribute to the poisoning of catalytic converters. For this reason the investigation focuses on organic friction modifier additives consisting only of carbon, hydrogen, oxygen and nitrogen, thus conforming to the so called 'CHON' philosophy. These additives are thought to have a beneficial effect on the vehicle emissions as they are not thought to contribute to catalytic converter poisoning.

The additives detailed in Table 3-3 were each selected for specific reasons. Together they allow the comparison of effects of chain length, saturation, chemical structure and reactive end head structure on the ability to influence friction behaviour in the contact. Stearic, Palmitic and Linoleic acid represent the fatty acid group and are all polar molecules with the same reactive end, and carbon chains of $\mathrm{C}_{18}, \mathrm{C}_{16}$ and $\mathrm{C}_{18}$ respectively. Both Stearic and Palmitic acid are saturated, whereas Linoleic acid is a polyunsaturated fatty acid, and as such has two double bonds within its hydrocarbon structure Table 3-3.

Stearamide, Behenamide and Erucylamide represent the amide group with Nitrogen containing polar heads and hydrocarbon tails of $\mathrm{C}_{18}, \mathrm{C}_{22}$ and $\mathrm{C}_{18}$ respectively. In this case Erucylamide has an unsaturated configuration with a double bond within its hydrocarbon tail.

In order to investigate trends in chemical species, it was necessary to look at a triglyceride formation. Tristearin was selected for this purpose, and essentially represents a variant of the Stearic acid molecule, in formation with a glycerol molecule. The molecular weight of this molecule is large with respect to other molecules, and its polar-end configuration inherently different to the acid or amide species.

The acetic acid alkyl esters again represent a differently structured molecule, whose attachment to the surface was thought to be significantly different compared with other molecules selected for investigation. 


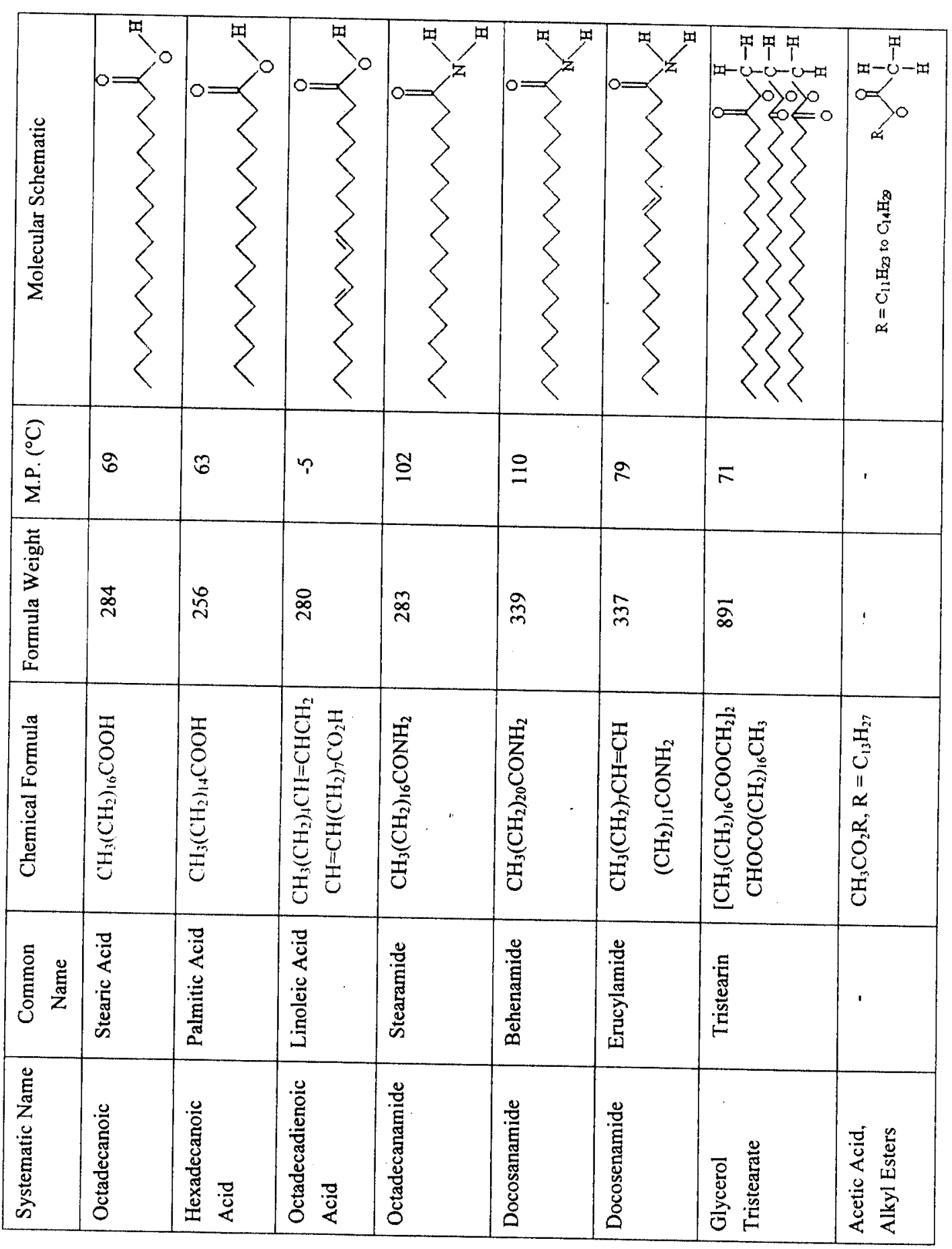




\subsection{Reciprocator Machine Tests}

\subsubsection{Additive Screening Test}

The main aim of the investigation using the Cameron Plint TE-77 High Frequency Reciprocator Tribometer was to screen the performance of numerous friction modifier additives. In order to do this it was necessary to develop a standardised test methodology which could be applied to all additives under investigation, thus permitting their direct comparison. As well as being scrutinised in their ability to reduce the coefficient of friction associated with the contact, it was also a specification of the test design that the speed of the additive reaction with the surface be investigated. For this reason the reciprocator machine was fitted with a bespoke oil circulation system which permitted the changeover from a sump containing pure base oil, to one containing $2 \mathrm{wt} \%$ additive in base oil without disruption to the test (Figure 3-3).

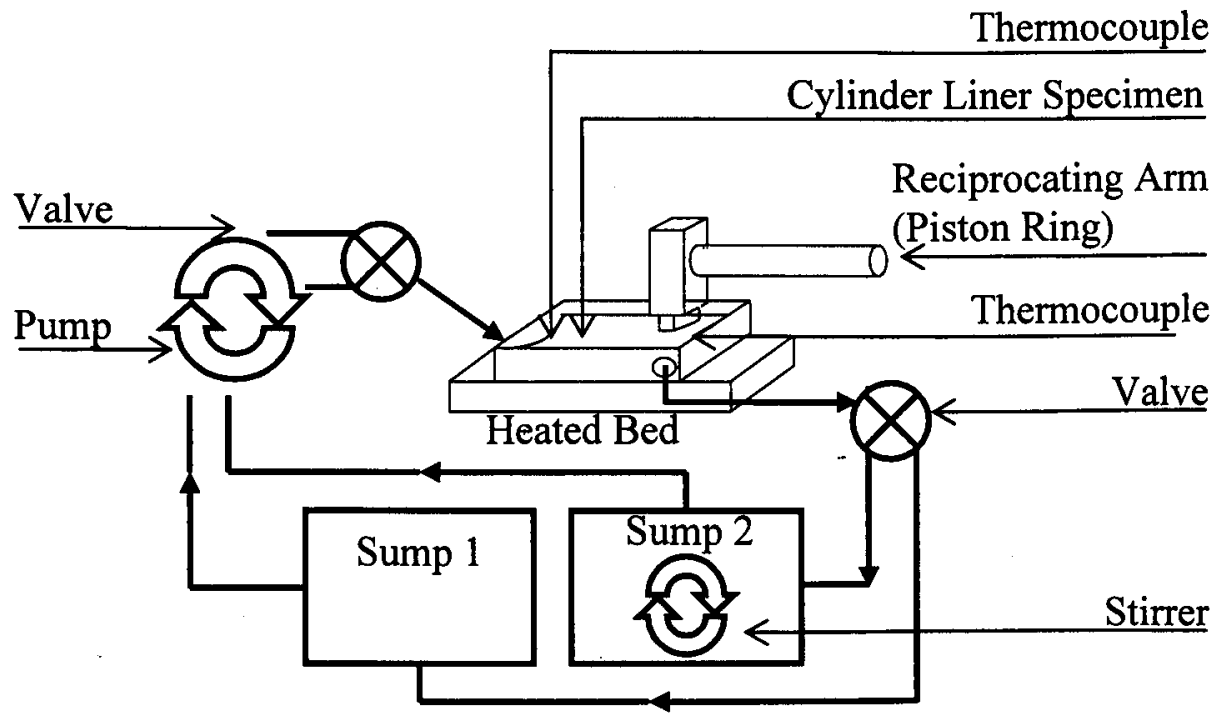

Figure 3-3 Schematic Representation of Reciprocator Setup

The oil feed system employed utilised a peristaltic pump fitted with two parallel feed pipes ensuring flow was provided to the system from two sumps, thus facilitating the change-over of one feed to the other mid-test. This system ensured the oil feed rate was identical from one source to the other, eliminating it as a factor for consideration when comparing data sets. Sump one and two were located sideby-side and were both subject to ambient temperatures and pressures prior to pumping the working section of the machine. Sump two was placed on a magnetic stirrer throughout the tests to ensure homogeneity of the base oil / additive blend prior to being fed to the contact. 
Lubricant enters the system on the left hand side of the contact through a nozzle directing the flow over the cylinder liner surface. Once it has passed through the contact, the lubricant is extracted from the lubricant reservoir and returned to the sump by a second peristaltic pump running at a scavenging pace.

The machine was run at the conditions detailed in Table 3-4, which were selected to simulate the conditions found at the TDC reversal point within a firing gasoline engine.

\begin{tabular}{cc}
\hline Parameter & Condition \\
\hline Stroke Length & $5 \mathrm{~mm}$ \\
\hline Drive Motor Speed & $2000 \mathrm{rpm}$ \\
\hline Load & $150 \mathrm{~N}$ \\
\hline Liner Specimen Surface Temperature & $110^{\circ} \mathrm{C}$ \\
\hline Oil Pump Flow Rate & $1 \mathrm{~L} / \mathrm{hr}$ \\
\hline
\end{tabular}

Table 3-4 Additive Screening Test Conditions

- The pump flow rate was set at 1 litre/hr, and practically manifested itself as a drip feed. This speed was selected as one litre of each additive blend was created for use in the screening tests, thus giving each molecule of additive a chance to pass through the contact once. However, due to mixing" in the sump it is accepted that this was probably not the case. Should passing through the contact deteriorate or destroy additive molecules, having one litre of lubricant ensured there would always be active molecules entering the contact.

- A stroke of $5 \mathrm{~mm}$ was selected to ensure the system operated predominately in the boundary lubrication regime for the chosen conditions, the shorter stroke not allowing the system to enter the mixed or hydrodynamic lubrication regimes. A short stroke also ensures greater alignment between specimens throughout the cycle, limiting harsh vibrations which are often experienced with long stroke lengths.

- A drive motor speed of $2000 \mathrm{rpm}$, corresponding to a reciprocating frequency of $33.3 \mathrm{~Hz}$ was selected for the tests. Given the stroke length of $5 \mathrm{~mm}$, this practically manifests itself as an average speed of 0.33 $\mathrm{m} / \mathrm{s}$. This speed was primarily selected to ensure the contact stayed in 
the boundary lubrication regime, but was also selected as preliminary tests revealed the speed was ideal for minimising noise in this particular system.

- Thermocouples mounted on the surface of the cylinder liner were used to ensure the surface temperature of the cylinder liner was maintained at $110^{\circ} \mathrm{C}$. This temperature was selected as it is typical of the TDC cylinder liner temperature measured in fired engine tests presented in other chapters of this thesis.

- A normal load of $150 \mathrm{~N}$ was applied to the reciprocating arm. This load was used to simulate a 'worst case scenario' at the top ring zone, where data taken from the Ricardo Hydra engine at full load and 2000 rpm suggests a peak combustion cylinder pressure of approximately 4.0 $\mathrm{MPa}$. This pressure corresponds to a testing load of approximately 150 $\mathrm{N}$ given the contact geometries of the specimens. The workings detailing the calculation of required force are included in the Appendices, section A1.

To achieve the secondary aim of the research; to investigate the speed of reaction of the additives and to investigate the additives films tenacity, a series of parametric tests were conducted on the additives, ensuring data resolution was given in areas where it was deemed necessary. The following details the additive screening test procedure developed as a result of parametric testing:

The machine was stabilised at the given running conditions prior to activation of the data recorder. Once stabilised, the machine was run for thirty minutes with lubricant feed supplied from sump 1 containing pure XHVI-4 ${ }^{\mathrm{TM}}$ (Shell) base oil. Data captures were then taken at ten minute intervals. Without disruption to the running of the machine, the lubricant supply was then switched to feed from sump 2 , containing $2 \mathrm{wt} \%$ friction modifier in XHVI-4 ${ }^{\mathrm{TM}}$ (Shell). Simultaneously data capture resolution was increased to one per minute for a ten minute period. At this point the lubricant leaving the working area was pumped to waste for five minutes before being reconnected to the feed sump. This was done to ensure cross contamination of the feed sump was minimal. The data capture interval was then reverted back to 10 minutes until the additive had been in the system for $1 \mathrm{hr}$. At this point the lubricant feed was switched back to that of sump 1, and data resolution refined to one data capture per minute for the next 10 minutes. After this period, and still running on pure base oil, the data capture interval was set at 10 minutes until a total test time of 2 hours 10 minutes had been reached. 
Following the completion of a test, the machine was allowed to cool before isopropanol was flushed through the system for 1 minute. The machine was then run at the given conditions for a thirty minute period on pure XHVI-4 ${ }^{\mathrm{TM}}$ (Shell), with lubricant exiting the working area going to waste. Due to heavy contamination of the sump 2 feed line, this was changed prior to each test with a new additive.

\subsubsection{Additive Package Formulation and Blending Test}

Guided from the results of the preceding additive screening tests, a set of tests was conducted to investigate the effects of additive package formulation and blending. In the conventional sense, lubricant additive package formulation is considered to be the creation of an additive package which caters to all the requirements of the lubricant specification, and would typically contain a variety of chemicals each with a separate purpose and function. However, in this work, the specification of the lubricant formulation is solely to provide the most effective friction modifier performance, and as such the additive package need only consist of friction modifier. This work pushes the boundary of additive package formulation as it conjects the hypothesis that an additive package should not only consist of a single chemical whose purpose it is to reduce friction, for example, but may contain a number of carefully selected chemicals chosen to perform together on the same task, each with an exceptional performance in the area in which they might act. For example, the combination of an additive with capability of reducing end-of-stroke friction with one which can reduce mid-stroke friction would lead to even lower friction operation.

A test was conducted to investigate the potential performance benefit of blending high performance friction modifier additives in a formulation which gives a performance in excess of that achievable through any of the constituent additives on their own. The test structure and operating conditions were the same as those described in section 3.3.1, Additive Screening Tests, the difference being that sump two contained a blend of additives rather than a single additive in XHVI-4TM (Shell).

\subsubsection{Additive Concentration Tests}

A set of tests was conducted to investigate the effects of additive concentration on friction in the piston ring / cylinder liner contact. These tests were conducted using Linoleic Acid as it is the only additive under investigation which is liquid at ambient temperatures and facilitates easy and completely homogeneous blending. Concentrations of $0.25 \%, 0.5 \%, 1 \%, 2 \%$ and $4 \%$ Linoleic acid in XHVI-4 ${ }^{\mathrm{TM}}$ (Shell) were investigated. The test structure and conditions were as described in 3.3.1, whereby a baseline coefficient of friction was recorded for 30 minutes before additive was introduced to the contact at a given concentration for 1 hour. After one 
hour of additive influenced action, 40 minutes of operation on pure XHVI-4 ${ }^{\mathrm{TM}}$ (Shell) was recorded. As the tests were all identical in nature, and were performed using the same piston ring and cylinder liner specimens, the only variable was the concentration of Linoleic acid in sump 2. This test design permits the direct comparison of coefficient of friction reduction as a result of additive concentration variation.

\subsubsection{Piston Ring Type Test}

As discussed in section 3.1.2, the typical top compression ring of the GM 2.0 litre piston ring pack consists of a grey cast iron substrate phosphated with chrome inlay and finished with a barrel face. However, a piston ring-pack with a top compression ring whose configuration differs greatly from the conventional is also available. The piston ring features a full face molybdenum flame sprayed wear resistant coating (Figure 3-4).

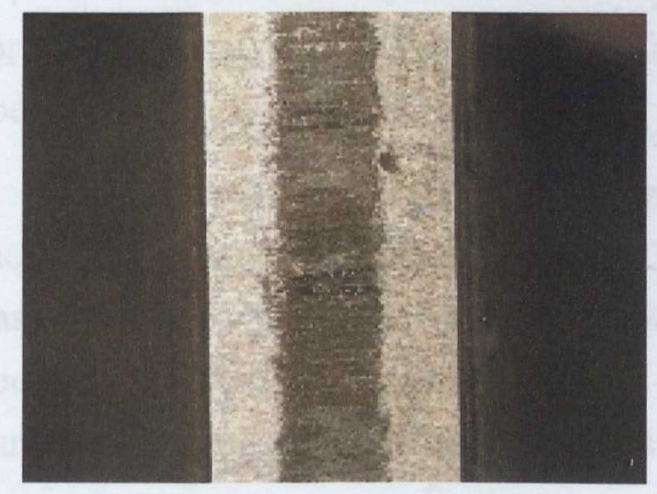

Chrome Ring Ra Worn $=0.078 \mu \mathrm{m}$

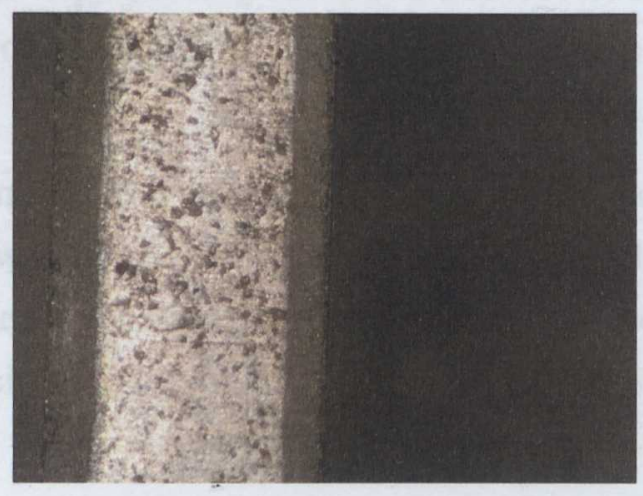

Molybdenum Ring $\mathrm{Ra}$ Worn $=0.45 \mu \mathrm{m}$

Figure 3-4 Photograph of Chrome Coated and Molybdenum Coated Piston Rings

Given that there are two types of top compression ring available, it was considered of scientific interest to investigate the effects of the differing configurations of piston ring.

A series of tests was conducted whose methodology was exactly the same as that described in section 3.3.1, the only difference being the type of piston ring section used. However, due to time and material constraints it was only possible to investigate the effects using three additive formulations. Blends containing $2 \mathrm{wt} \%$ Stearic acid, Palmitic acid and Linoleic acid were selected for investigation, as together they permit the examination of chain length and bond saturation on friction modifier performance. 
To give further insight into the mechanism of film formation when running with molybdenum rings, a further test was conducted at $60^{\circ} \mathrm{C}, 40 \mathrm{~N}$ and $2000 \mathrm{rpm}$, conditions which meant the machine was running on the cusp of hydrodynamic lubrication. Data capture was taken approximately every 10 seconds to capture in detail the action of the $2 \mathrm{wt} \%$ Stearic acid formulation as it entered the contact.

\subsubsection{Film Creation Tests}

As well as being the ideal machine to accurately screen the performance of the candidate friction modifier additives, the TE-77 provides a platform on which to create additive films for surface analysis. The big advantage of creating films using the TE-77 is that it can use sections of real engine components as the running specimens, thus replicating the contacting surfaces as closely as is possible. The only downside to films created using the TE- 77 fitted with engine component specimens is that they are not ideal specimens for surface analysis. The cylinder liner section possesses two undesirable properties; it is not only relatively dull in appearance, but is also cylindrical in shape. The piston ring section is also not ideal for surface analysis. Inherently it is far from being flat, a valuable property of specimens for surface analysis.

The machine was fitted with new piston ring and cylinder liner specimens and the run-in procedure detailed in Table 3-2 was conducted with XHVI-4 ${ }^{\mathrm{TM}}$ (Shell) base lubricant. This procedure was developed to ensure a suitable running surface had been created on the cylinder liner specimen to permit both the formation of surface films and their subsequent analysis.

Once run in, the oil supply was switched to sump two containing 2 wt\% additive, and run at $2000 \mathrm{rpm}, 110^{\circ} \mathrm{C}$ and $150 \mathrm{~N}$ for two hours in order to give the system time to lay down an effective surface film. At this point, the load of the machine was gradually decreased until it was almost negligible before the machine was stopped. This was done as the tenacity of the films was unknown and it was thought the films might be removed should the machine be stopped with the usual $150 \mathrm{~N}$ applied load. Once removed from the machine, the components were not placed in an ultrasonic water bath in solvent as would be the usual practice prior to surface analysis, but were simply blown over with dry compressed air to remove the bulk lubricant from the respective surfaces. The specimens were then placed in air tight bags, and in air tight containers, before being placed in the refrigerator for storage. This seemingly unorthodox method of surface preparation prior to analysis was decided upon for a number of reasons. Firstly as the specimens were not ideally suitable for surface analysis such as X-ray Photon Spectroscopy, which requires small flat specimens with ultimate cleanliness for the high vacuum measurement 
environment, it was decided that the specimens would be ideal candidates for Fourier Transform Infrared (FTIR) microscopy, a surface analysis technique presented in more detail in section 3.5.1. This type of microscopy is non-intrusive and is capable of showing areas on the surface where additive films form, be they chemically or physically adsorbed. As it was not known if the films manifested themselves in the physically adsorbed form, it was believed that vigorous cleaning methods might remove them entirely from the surface. Simply blowing the surfaces with dry compressed air seemed a reasonable method for removing excess lubricant, whilst leaving any surface films intact.

Tests were conducted using three formulations, Linoleic Acid, Erucylamide and a blend of the two. The test involving Linoleic acid was repeated with a molybdenum coated piston ring instead of a chrome coated section.

\subsection{Test Lubricant Formulations}

In order to truly simulate the conditions at the top ring zone of a firing engine, it is essential that the lubricant found in this area be mimicked as closely as possible. It is was shown in Chapter 2 that a great deal of fuel dilution is present in the top ring zone lubricant, a fact which underlies the potential success of gasoline containing friction modifier. It is hypothesised that the repeated firing of the fuel injector would continually supply fresh friction modifier to the top ring zone, where it mixes with the cylinder liner lubricant and reacts with the cylinder liner and piston ring surfaces. Ideally, bench-top tribometer tests would have been conducted on gasoline doped lubricant samples, but this was not feasible as it is extremely dangerous to interject volatile substances such as gasoline into contacts with elevated temperatures. Even if the test temperature was lowered, local contact temperatures could have reached a high enough level to cause ignition. However, it is not acceptable to simply ignore the presence of fuel in the lubricant sample, as it serves to reduce the bulk viscosity of the lubricant, thus changing the contact mechanics. It was for this reason that XHVI-4TM (Shell) was selected as the bulk lubricant for this investigation. This is a low viscosity base lubricant, and may be considered to act like a typical SAE 5W-30 engine lubricant with approximately $30 \%$ fuel dilution. This figure was based on a calculation using the blending law presented by Prausnitz as detailed below (Reid, Prausnitz et al. 1977).

$$
\ln n_{m}=x_{f} \ln n_{f}+x_{o} \ln n_{o}
$$

It was decided to dope the lubricant with $2 \mathrm{wt} \%$ friction modifier additive in each blend. This high treat level was selected for the following reasons: 
The friction modifiers under investigation were selected for their ability to form films on metal surfaces with which they come into contact. If a low concentration of additive were used in the tests, it is conceivable that all additive might react on metal surfaces other than those of the contact under investigation. This would not be a problem in the engine environment as the repeated firing of the injector would constantly administer new additive to the surface. In addition, preliminary investigation revealed that some of the additives which are solid at room temperature can partially fall out of suspension once blended in base oil, thus having a suitably high concentration of additive ensured the contact remained saturated with additive throughout the test. The blending procedure for additives which are solid at room temperature involved heating the solution of base oil and additive to a temperature in excess of that of the additive melting point whilst continually stirring the mixture.

\subsection{Post Test Specimen Analysis}

On completion of the film creation tests, section 3.3.5, the cylinder liner and piston ring specimens were blown over with dry compressed air and stored in air tight bags in a refrigerator prior to surface analysis.

\subsubsection{Fourier Transform InfraRed Microscopy (FTIR)}

When exposed to an infrared beam, chemical bonds absorb infrared energy at specific frequencies (or wavelengths). The spectral locations of the infrared absorptions can be used to ascertain information regarding the chemical compounds within a molecule. During microscopy mode, a beam of infrared is focused on a reflective specimen, where the surface film of the specimen absorbs a portion of the energy. The energy reflected by the specimen is then recorded by a sensitive detector capable of detailing the wave numbers at which specific absorptions occurred. The infrared energy is low compared to optical, and for example X-ray, frequencies and hence the penetration of the beam is such that only the first few microns of the specimen surface are analysed by the machine, making the technique ideal for looking at thin organic surface films (O'Connor, Sexton et al. 2003).

As discussed earlier, the dull cylindrical nature of the cylinder liner specimens and the highly curved nature of the piston ring surfaces make them far from ideal for surface analysis, however, FTIR analysis of the surfaces was conducted on the specimens both on and away from the worn area. Measurements were conducted at the centre of the wear track area on both piston ring and liner specimens. The FTIR analysis was conducted by the author at the University of Leeds using a Nicolet FTIR Microscope with a relatively large 500 micron square spot size. 60 scans of 
the surface were taken at a given point from which an average was taken, thus filtering out any noise and sharpening the peak definition.

In addition to the FTIR analyses conducted at the University of Leeds, a set of specimens was selected for in-depth FTIR analysis which took place at Perkin Elmer (UK). In this case analysis was conducted using a Spotlight 200 series FTIR automated microscope spectrometer system, which was set to perform scans along a line which encapsulated data from both the worn and unworn regions. The spatial resolution of the system was set at 100 microns, with a spectral resolution of $8 \mathrm{~cm}^{-1}$, and spot size of $100 \mu \mathrm{m}^{2}$. 25 scans were conducted at each site and were used to create an average.

\subsection{Reciprocator Results Analysis}

\subsubsection{Additive Screening Tests Results Analysis}

Figure 3-5 displays the coefficient of friction results from the additive screening tests. On inspection of Figure 3-5 it is immediately apparent that the additives perform in a number of different ways. Repeatability tests conducted revealed the screening tests were highly repeatable, allowing the tests to be analysed to a high degree of resolution. The accuracy of the tests was revealed to be high enough to give significant meaning to the finest detail of the characteristic shapes associated with different additives action. A calculation based on repeatability tests suggests the error associated with repeatability of the tests is $2 \%$.

In each test it is clear where the oil supply was switched to one containing friction modifier additive, as there is an appreciable drop in measured coefficient of friction. The resolution of the data capture at this point, and for the proceeding ten minutes, is such that the shape of the curve at this point gives a good indication as to the development of the additive film. After this period, where the sampling resolution returns to 1 data capture every ten minutes, knowledge can be gained regarding the stability and time related formation of films. At the 90 minute mark, where the oil supply was switched back to that of pure XHVI-4 ${ }^{\text {TM }}$ (Shell), the sampling resolution returns to 1 per minute for the proceeding 10 minutes, allowing information to be gained regarding the removal of the formed film, or conversely the tenacity.

It is clear that the performance of the Acid additives is similar; all acid additives exhibiting a relatively slow formation stabilising after approximately 10 minutes. The formation curve associated with the Linoleic acid suggests film formation takes longer than with saturated acid molecules, but allows the contact to 
have lower friction operation. The removal of acid films appears to be rapid in all cases, with a return to near the baseline coefficient of friction in a matter of minutes. Again the polyunsaturated molecule, Linoleic acid, differentiates itself from the saturated molecules with a visible spike in coefficient of friction once fresh additive was removed from the contact. After this spike there is notable instability in the coefficient of friction value.

All amide additives appear to perform similarly. Their characteristic transitions are almost the exact mirror image of that of the acid molecules. Their formation on the surface is extremely rapid taking approximately one minute to form what appears to be a stable film. As is the case with the acid tests, the unsaturated molecule differentiates itself from the performance of the saturated molecules with an extended additive film formation period, leading to ultimately lower friction operation. On switching from additive doped lubricant to running on pure XHVI$4^{\mathrm{TM}}$ (Shell) the additive films are removed more slowly than they formed, and as with the acid films, the coefficient of friction does not quite return to that of the original baseline.

The performance of Tristearin and the Acetic Acid Alkyl Esters are similar with both additives showing poor frictional performance but appear to form quite tenacious films which are not easily removed simply by reverting to pure XHVI-4 ${ }^{\mathrm{TM}}$ (Shell) lubrication.

Figure 3-6 details the performance of the additives based on a calculation considering the reduction of coefficient of friction between the measurement at 30 minutes, and that taken at 90 minutes. Looking at the fatty acid additive performance, it is clear that the unsaturated molecule, Linoleic acid, performs better than the saturated molecules with a $53 \%$ reduction in coefficient of friction.

Typically the performance of the amides was poor when compared with that of the acid group with the exception of the unsaturated variant Erucylamide, whose maximum reduction in coefficient of friction was $48 \%$. When looking at the response of Stearamide and Behenamide it appears Behenamide performs most competently at reducing friction, reducing the coefficient of friction by $36 \%$ as opposed to $30 \%$. 

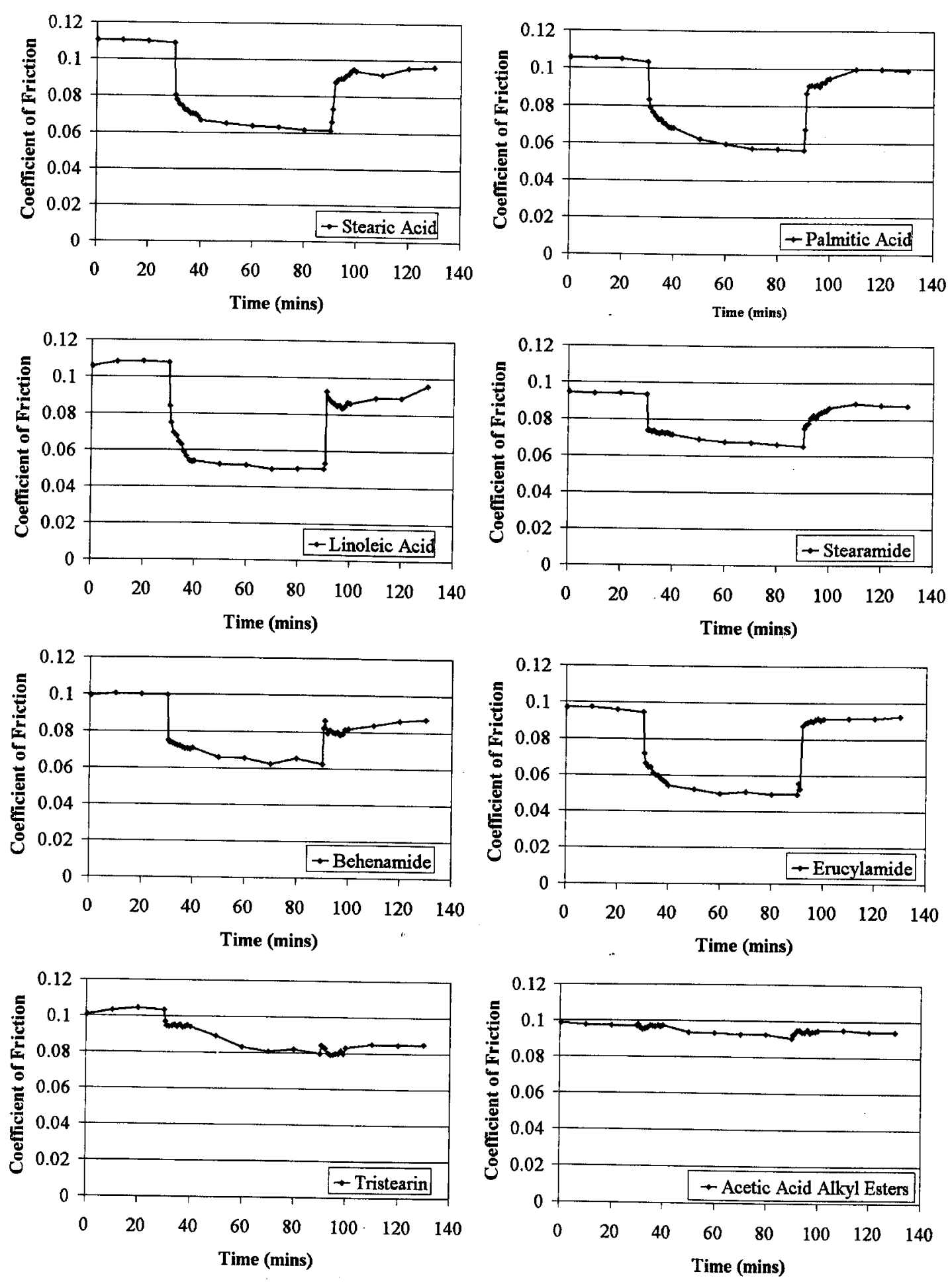

Figure 3-5 Additive Screening Test Coefficient of Friction Results

The performance of Tristearin and the Acetic Acid Alkyl Esters was poor, reducing the coefficient of friction by just $23 \%$ and $7 \%$ respectively. However, it is worth noting that this figure only compares the performance of the additives in terms of reduction of friction, and does therefore not highlight the apparent superior tenacity of the additive films. 
On inspection of Figure 3-7 it is clear that there is a distinct difference between the curves representing the performance of unsaturated molecules compared with that of saturated molecules. The trend appears to be similar for both acid and amide, and shows that the initial response of the additive, after the 30 minute mark, is heavily dominated by the saturation of the reacting molecules'. It is shown that the unsaturated molecules have a greater ability to reduce friction in the contact than saturated molecules.

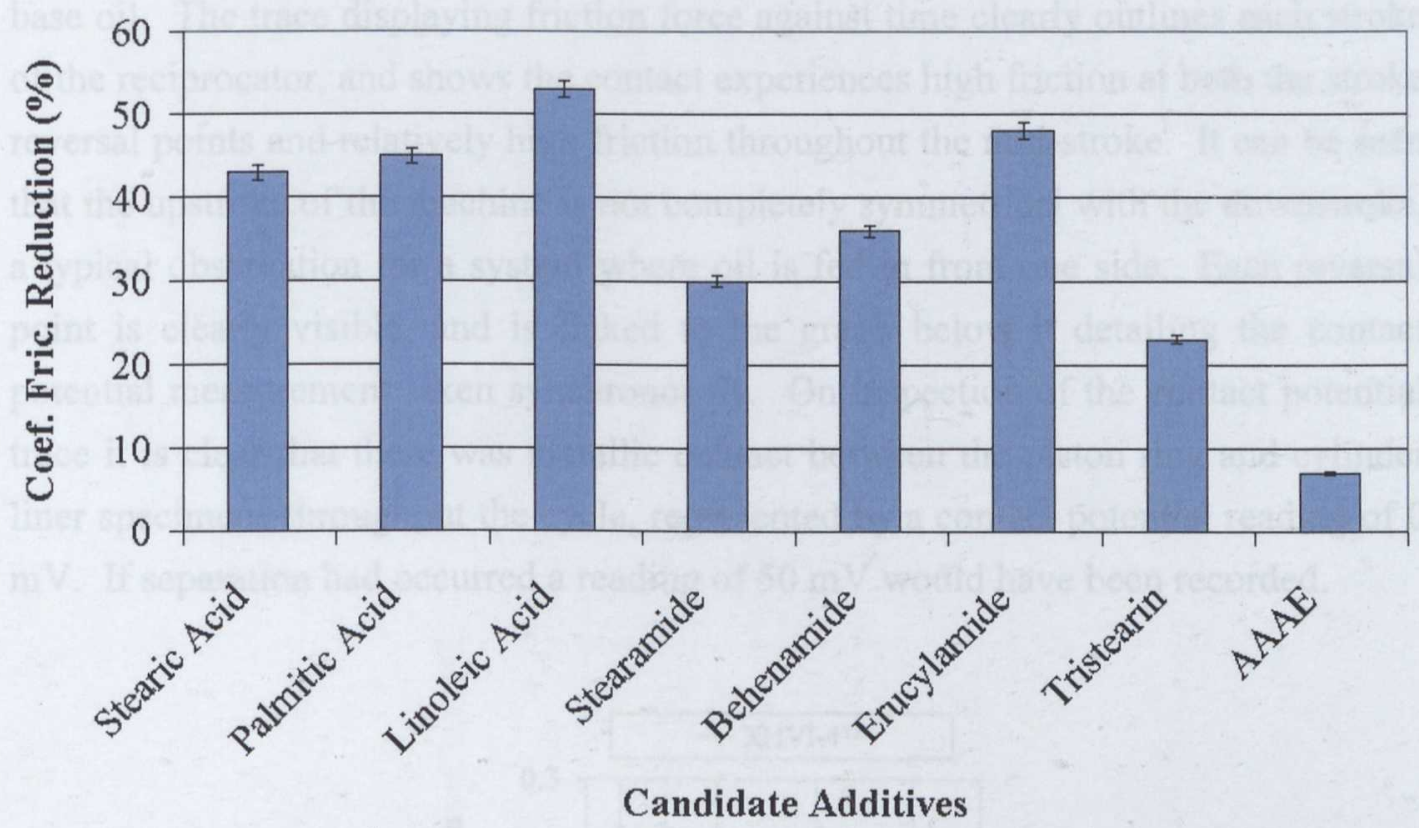

Figure 3-6 Reduction in Coefficient of Friction
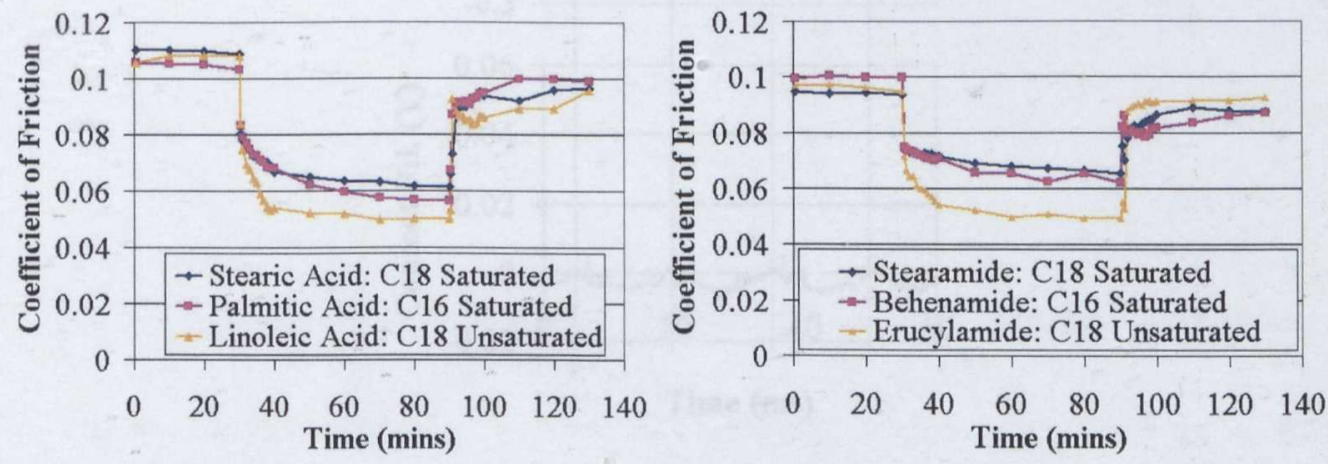

Figure 3-7 Performance Related to Saturation of Chemical Bonds

In addition to the coefficient of friction data, the instantaneous friction and contact potential data captured during the tests gives us a large amount of information regarding the action of the additives. Although the coefficient of friction, which is calculated from the instantaneous friction force, can give us an 
indication of the bulk friction condition for a given contact, analysis of the raw data gives us an insight into the mechanism underpinning the friction reduction mechanism itself. Graphs presented below are each the result of a synchronous data capture at a sampling rate of $100,000 \mathrm{~Hz}$, and taken after the additive had been in the machine for one hour. The top graph of each set displays the instantaneous friction force, and the bottom the contact potential measurement associated for a $60 \mathrm{~ms}$ or two full cycle data capture.

Figure 3-8 shows the raw data taken during a test using pure XHVI-4TM (Shell) base oil. The trace displaying friction force against time clearly outlines each stroke of the reciprocator, and shows the contact experiences high friction at both the stroke reversal points and relatively high friction throughout the mid-stroke. It can be seen that the upstroke of the machine is not completely symmetrical with the downstroke, a typical observation for a system where oil is fed in from one side. Each reversal point is clearly visible, and is linked to the graph below it detailing the contact potential measurement taken synchronously. On inspection of the contact potential trace it is clear that there was metallic contact between the piston ring and cylinder liner specimens throughout the cycle, represented by a contact potential reading of 0 $\mathrm{mV}$. If separation had occurred a reading of $50 \mathrm{mV}$ would have been recorded.

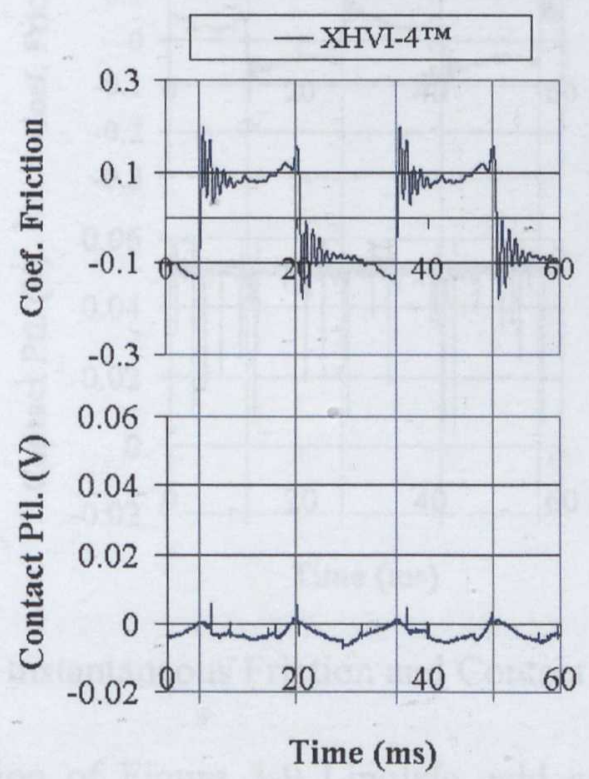

Figure 3-8 Pure Base Oil Instantaneous Friction and Contact Potential Measurement

The performance of the fatty acid friction modifier molecules is shown in Figure 3-9. It is apparent that the contact is experiencing very different lubrication conditions compared to those associated with running on pure base oil alone. It is clear that the friction created at the stroke reversal points is reduced considerably by the presence of the fatty acid molecules, and that the mid-stroke region also appears 
to have reduced friction operation. It is also noticeable that the stiction events evident in the friction trace taken with pure base oil have been reduced with the application of fatty acid friction modifier.
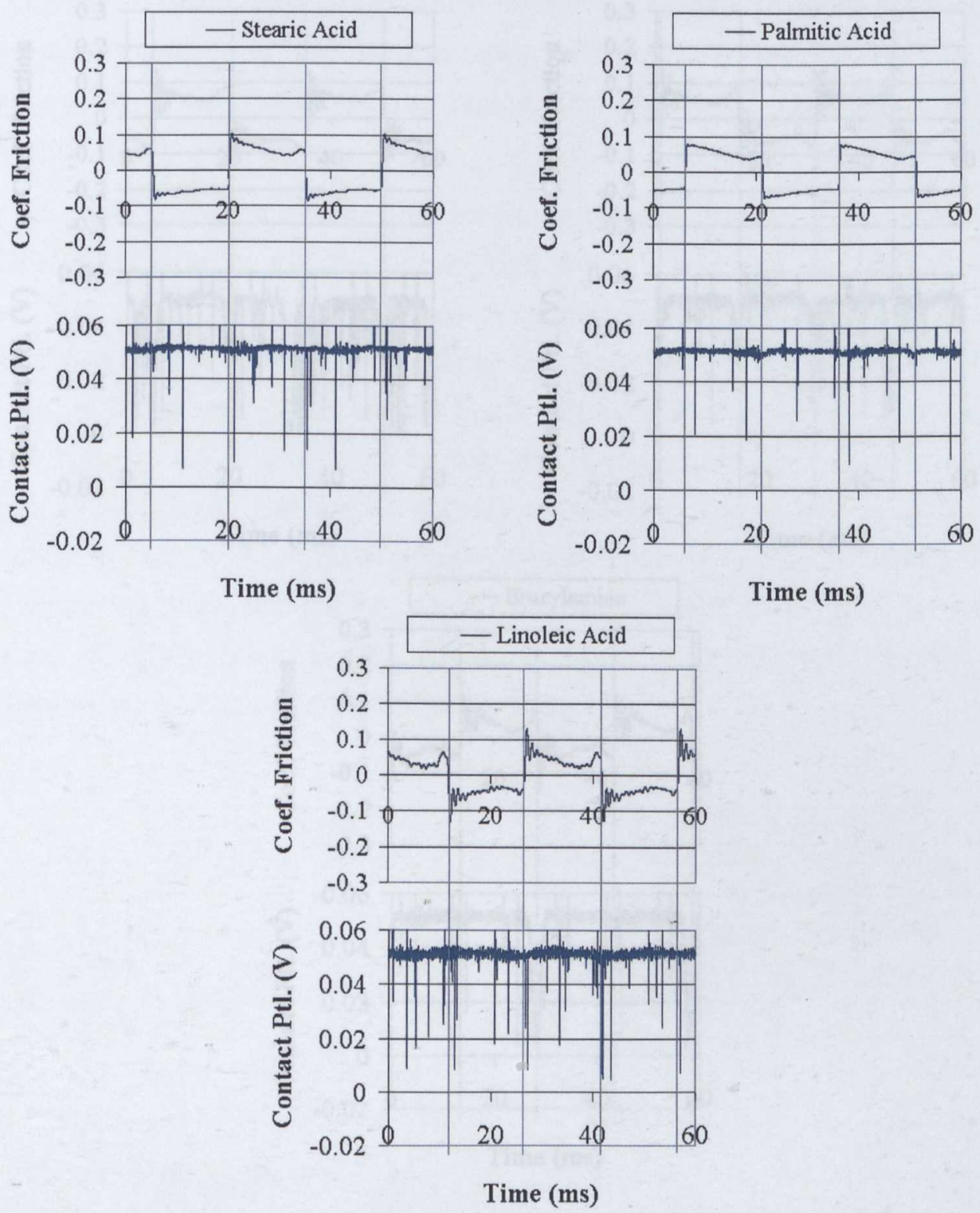

Figure 3-9 Fatty Acid Instantaneous Friction and Contact Potential Measurement

On closer inspection of Figure 3-9 Linoleic acid appears to have a further reduced mid-stroke friction when compared with both Stearic and Palmitic acid. It is this reduced friction in the mid-stroke region that is the cause for the enhanced performance of the additive when comparing coefficient of friction. All additives appear to form films capable of providing electrical insulation to the contact, suggesting metal-to-metal contact is scarce throughout the stroke. The film formed through application of Linoleic acid is possibly weaker or less dense than that 
formed by Stearic or Palmitic acid, as signs of metallic contact are evident at the stroke reversal points.
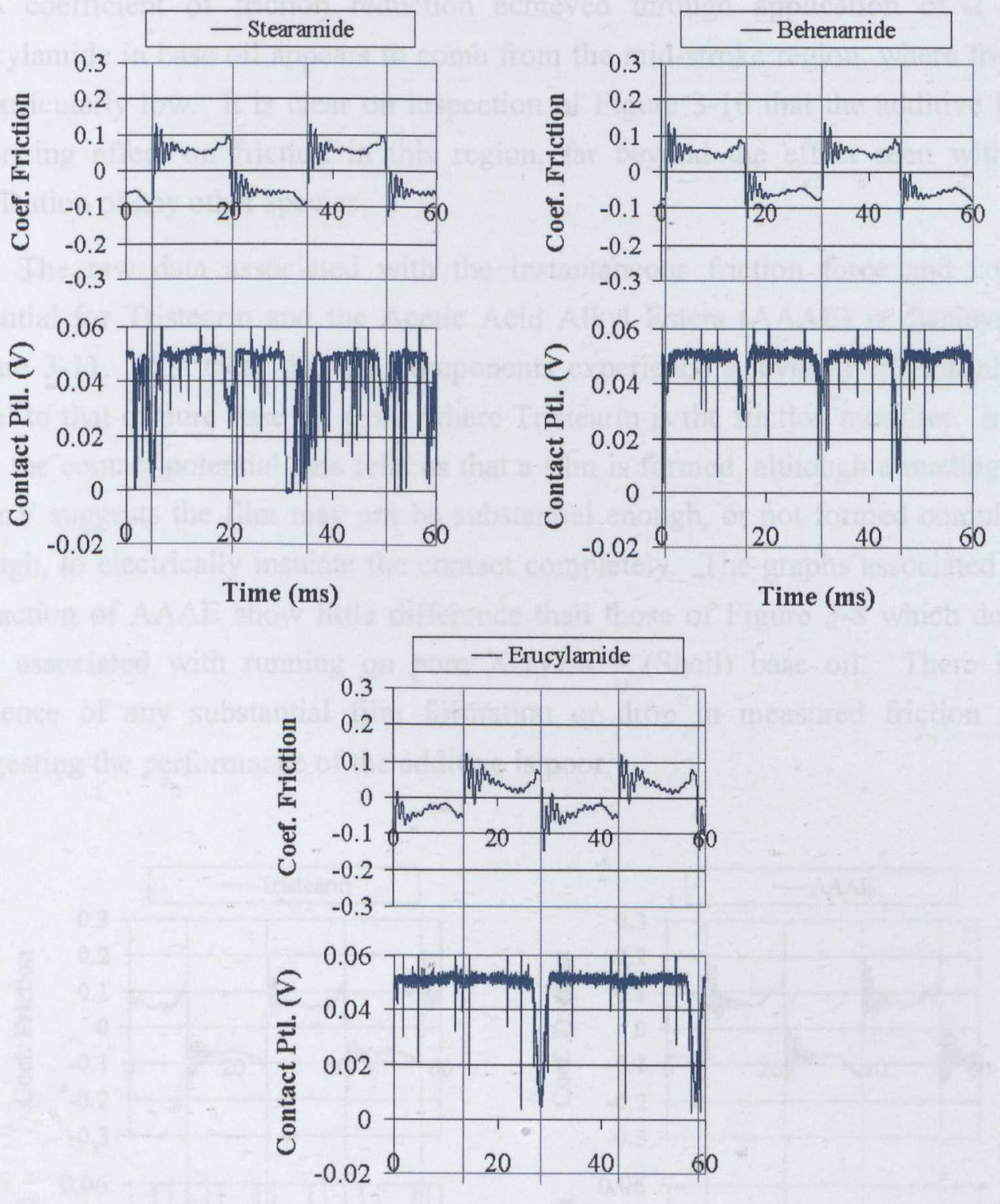

Time (ms)

Figure 3-10 Amide Instantaneous Friction and Contact Potential Measurement

Figure 3-10 depicts the instantaneous friction and contact potential results associated with the performance of the amide group of additives. Like the acid based friction modifiers, it is clear that the contact experiences low friction at reversal and mid-stroke points when compared with running on pure base oil alone. Of key interest in these figures are the contact potential traces. It seems the additive film formed in each case is weaker, or less complete, than that of the fatty acid group, as metallic contact is seen in greater proportion. It is interesting to note that the components experience more metal-to-metal contact when Stearamide is the 
friction modifier than when Behenamide is employed suggesting an influence of chain length on performance. Erucylamide provides the greatest level of electrical insulation suggesting the most complete film formation of all the amides tested. The $48 \%$ coefficient of friction reduction achieved through application of $2 \mathrm{wt} \%$ Erucylamide in base oil appears to come from the mid-stroke region, where friction is particularly low. It is clear on inspection of Figure 3-10 that the additive has a surprising effect on friction in this region, far beyond the effect seen with the application of any other species.

The raw data associated with the instantaneous friction force and contact potential for Tristearin and the Acetic Acid Alkyl Esters (AAAE) is displayed in Figure 3-11. It is clear that the components experience a level of friction almost equal to that of pure base oil alone where Tristearin is the friction modifier. In this case the contact potential data tells us that a film is formed, although a reading of $40 \mathrm{mV}$ suggests the film may not be substantial enough, or not formed completely enough, to electrically insulate the contact completely. The graphs associated with the action of AAAE show little difference than those of Figure 3-8 which depicts data associated with running on pure XHVI-4TM (Shell) base oil. There is no evidence of any substantial film formation or drop in measured friction force suggesting the performance of the additive is poor.

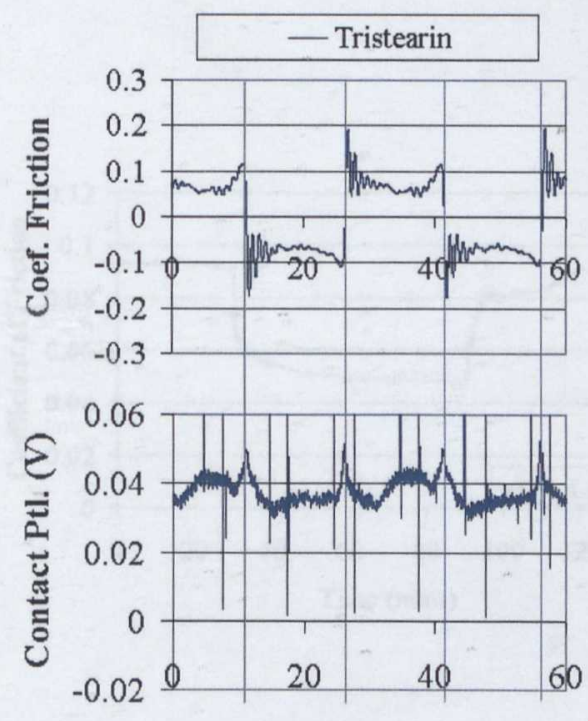

Time (ms)

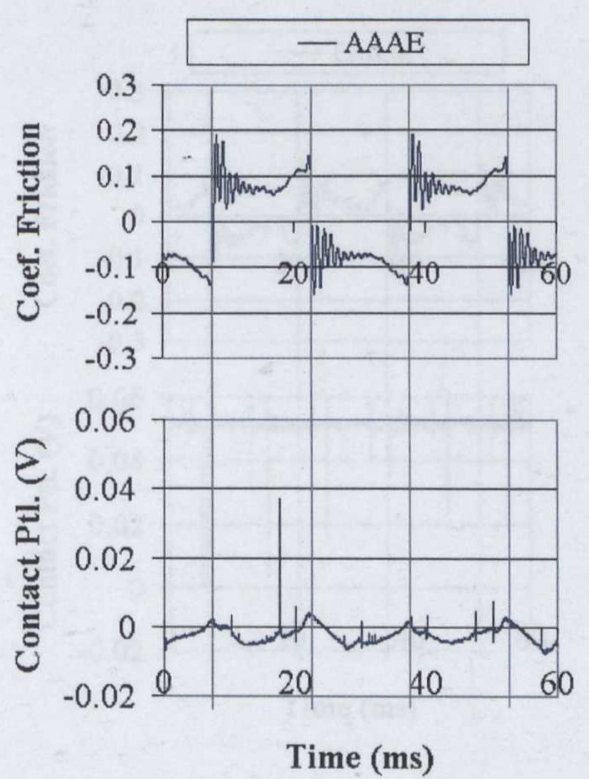

Time (ms)

Figure 3-11 Triglyceride and Ester Instantaneous Friction and Contact Potential Measurement 


\subsubsection{Additive Package Formulation and Blending Results and Analysis}

The following results summarise the performance 'EruLin', of a blend of 1 wt\% Linoleic acid and $1 \mathrm{wt} \%$ Erucylamide in XHVI-4TM (Shell). This blend was formulated as a result of the exceptional performance displayed by both additives in the additive screening tests 3.6.1; Linoleic acid showed exceptional performance at reducing friction at stroke reversal, whilst Erucylamide showed exceptional performance during the midstroke. It was hypothesised that a blend of the two might create a system providing low friction operation throughout both stroke reversal and midstroke.

The reduction in coefficient of friction for the blend was calculated to be $50 \%$. It is interesting to note that the reduction in friction for Linoleic acid and Erucylamide was $53 \%$ and $48 \%$ respectively, suggesting the situation within the contact is a product of the action of both chemical species (Table 3-5). Of key interest in the blending results are the instantaneous friction and contact potential results. Figure 3-12 clearly shows the blend can reduce friction at the stroke reversal points, and that mid-stroke friction is also very low. The contact potential information suggests a complete film was formed between the surfaces forcing complete electrical isolation between interacting surfaces suggesting metal-to-metal contact is at a minimum.
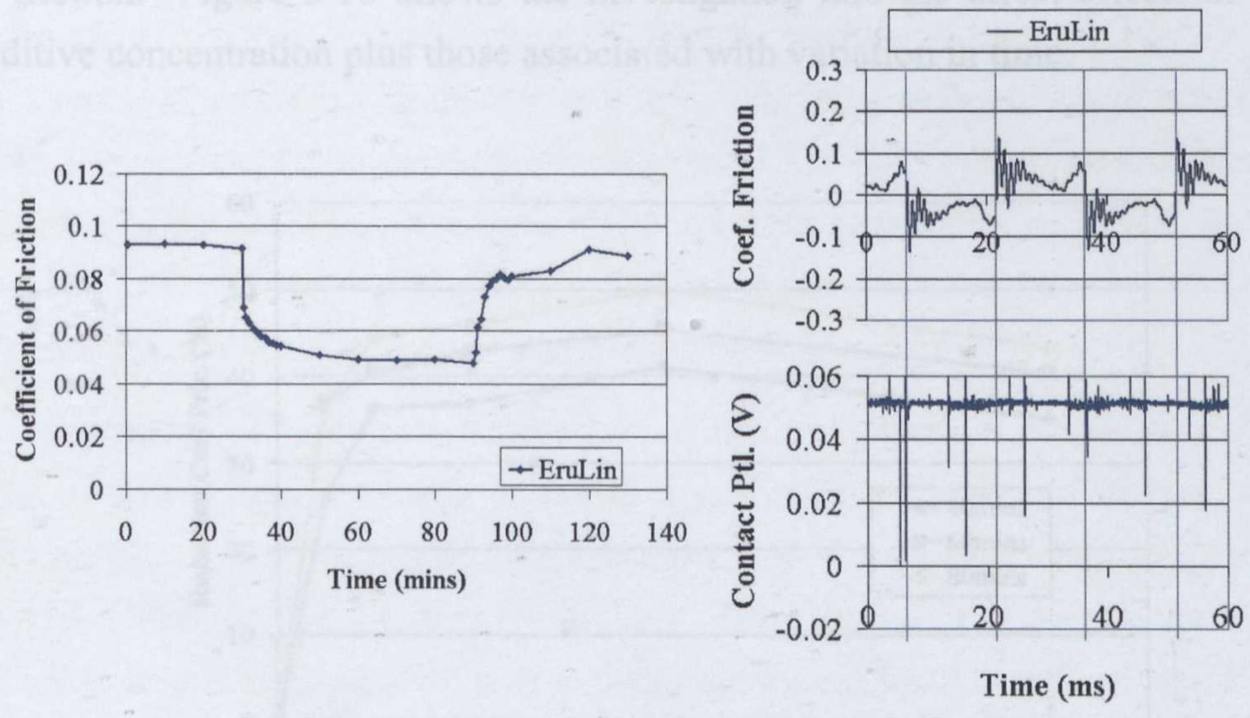

Figure 3-12 Additive Blending Results

Table 3-5 details both the reduction in coefficient of friction and the corresponding final coefficient of friction measured after one hour of additive influenced action. The table shows that although the blend of Linoleic acid and Erucylamide does not provide a greater reduction in friction coefficient than either of 
the additives individually, it does appear to reduce the final coefficient of friction measured in the contact, perhaps suggesting this is an important factor for consideration when analysing the performance of friction modifier additives.

\begin{tabular}{ccc}
\hline Blend & $\begin{array}{c}\text { Reduction in Coefficient } \\
\text { of Friction (\%) }\end{array}$ & $\begin{array}{c}\text { Final Coefficient of } \\
\text { Friction }\end{array}$ \\
\hline $\begin{array}{c}2 \mathrm{wt} \% \text { Linoleic Acid }+ \\
98 \mathrm{wt} \% \text { XHVI-4 }\end{array}$ & 53 & 0.05 \\
\hline $\begin{array}{c}\text { Twt } \% \text { Erucylamide }+ \\
98 \mathrm{wt} \% \text { XHVI-4 }\end{array}$ & 48 & 0.049 \\
\hline $\begin{array}{c}1 \mathrm{wt} \% \text { Erucylamide }+ \\
1 \mathrm{wt} \% \text { Linoleic Acid }+ \\
98 \text { wt } \% \text { XHVI-4 }\end{array}$ & 50 & 0.047 \\
\hline
\end{tabular}

Table 3-5 Coefficient of Friction Comparison

\subsubsection{Additive Concentration Investigation Results and Analysis}

To investigate the effects concentration has on friction modifier performance tests were conducted where the variable was the concentration of Linoleic acid in the base oil. Concentrations of $0.25 \mathrm{wt} \%, 0.5 \mathrm{wt} \%, 1 \mathrm{wt} \%, 2 \mathrm{wt} \%$ and $4 \mathrm{wt} \%$ were investigated, the results being displayed in Figure 3-13. The results presented show clearly that the concentration of additive has an effect on the reduction in coefficient of friction. Figure 3-13 allows the investigation into the direct effects of varying additive concentration plus those associated with variation in time.

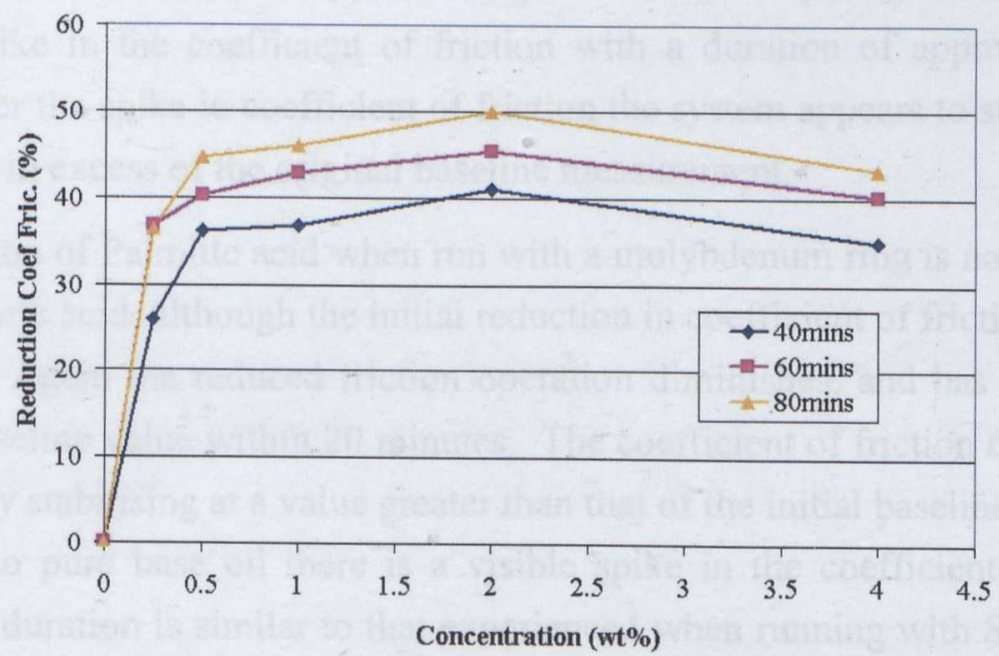

Figure 3-13 Additive Concentration Effects

On initial inspection of Figure 3-13 it is clear that time has an influence on the additives ability to reduce the coefficient of friction, the longer the additive is in the 
machine the greater the effect of the additive. The graph also shows that the optimum performance level for additive concentration is $2 \mathrm{wt} \%$, as the greatest coefficient of friction reduction is achieved by this blend. There is evidence to suggest that doping the lubricant with more than $2 \mathrm{wt} \%$ additive causes a decrease in the achievable level of friction reduction. Below concentrations of $0.5 \mathrm{wt} \%$ additive it is clear that performance drops off, suggesting this is the critical value of concentration associated with this specific test.

\subsubsection{Piston Ring Type Tests Results and Analysis}

The in-house standardised additive screening test presented in section 3.3.1 was repeated using a flame sprayed molybdenum coated ring instead of the usual chrome coating. Due to time and material constraints it was only possible to investigate the effects using three additive formulations. Blends containing $2 \mathrm{wt} \%$ Stearic acid, Palmitic acid and Linoleic acid were selected, as they permit the examination of chain length and bond saturation on friction modifier performance.

On initial inspection of Figure 3-14 the difference between the additive performance results taken with Chrome and Molybdenum coated piston rings is startling. The piston ring type appears to have a great influence on the measured friction when in the presence of fatty acid friction modifier. On consideration of the result depicting the friction coefficient for the Stearic acid blend, and running with a molybdenum ring, it is clear there is a friction reducing effect which reaches a maximum within the first few minutes after the additive enters the contact. This effect appears to diminish thereafter returning to a value close to that of the baseline within 30 minutes. Once the switch-back to pure XHVI-4TM (Shell) occurs there is a noticeable spike in the coefficient of friction with a duration of approximately 5 minutes. After the spike in coefficient of friction the system appears to stabilise to a value slightly in excess of the original baseline measurement.

The action of Palmitic acid when run with a molybdenum ring is not dissimilar to that of Stearic acid, although the initial reduction in coefficient of friction is not as pronounced. Again the reduced friction operation diminishes, and has returned to that of the baseline value within 20 minutes. The coefficient of friction continues to rise apparently stabilising at a value greater than that of the initial baseline. After the switch-back to pure base oil there is a visible spike in the coefficient of friction value, whose duration is similar to that experienced when running with Stearic acid. The test stabilises to a value very close to that of the original baseline.

Linoleic acid performs completely differently to both Stearic and Palmitic acid. The initial drop in coefficient of friction is small in comparison to the saturated molecules, but the additive continues to work further reducing friction in the contact 
for the full hour it is in the machine. At the end of the hour, the reduction in coefficient of friction is close to that initially achieved through application of Stearic or Palmitic acid. On return to lubrication by pure base oil alone there is some evidence of a spike in coefficient of friction, but values ultimately stabilise very close to that of the original baseline.
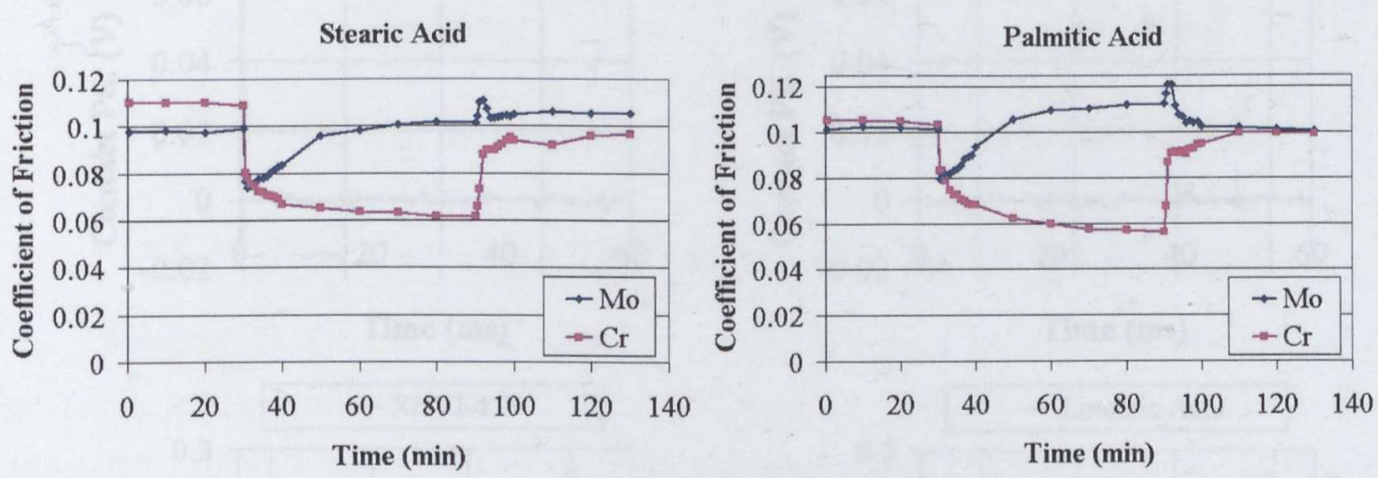

Linoleic Acid

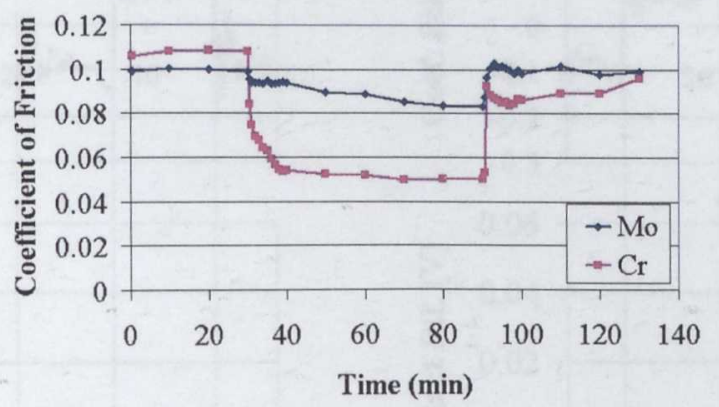

Figure 3-14 Piston Ring Material Coefficient of Friction Results

As was the case with the additive screening tests (section 3.6.1), the raw instantaneous friction and contact potential measurements taken during the tests aid understanding of the contact situation. The instantaneous friction and contact potential measurements taken at 60 minutes of additive influenced action from the TE-77 tests run with a molybdenum ring are shown in Figure 3-15. Coefficient of friction data analysed previously suggests that at 60 minutes of additive influenced action the lubrication conditions are that of a lubricant with no friction modifier. However, the friction force trace associated with Linoleic acid suggests some reduction in friction in the mid-stroke region.

Contact potential traces suggest almost complete metal-to-metal contact throughout the stroke of the machine. Interestingly, the measured level of the contact potential trace is lower and more stable than when run on pure base oil alone, where some variation in the value typically occurs. 


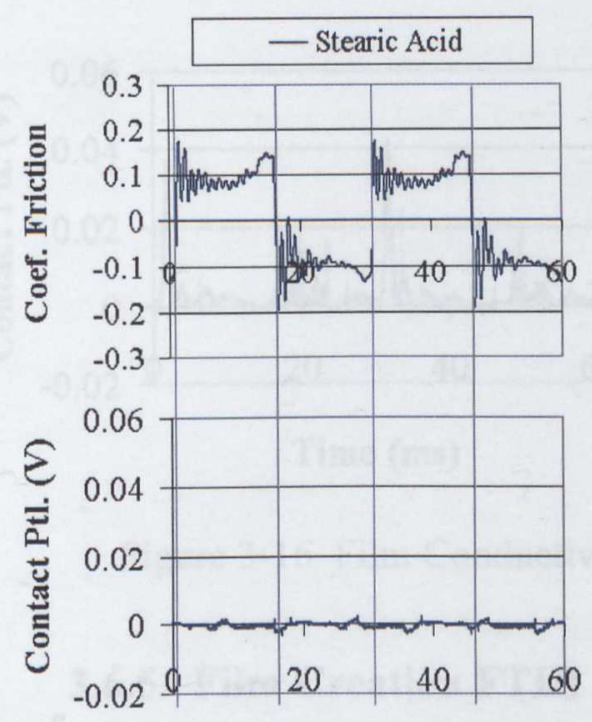

Time (ms)

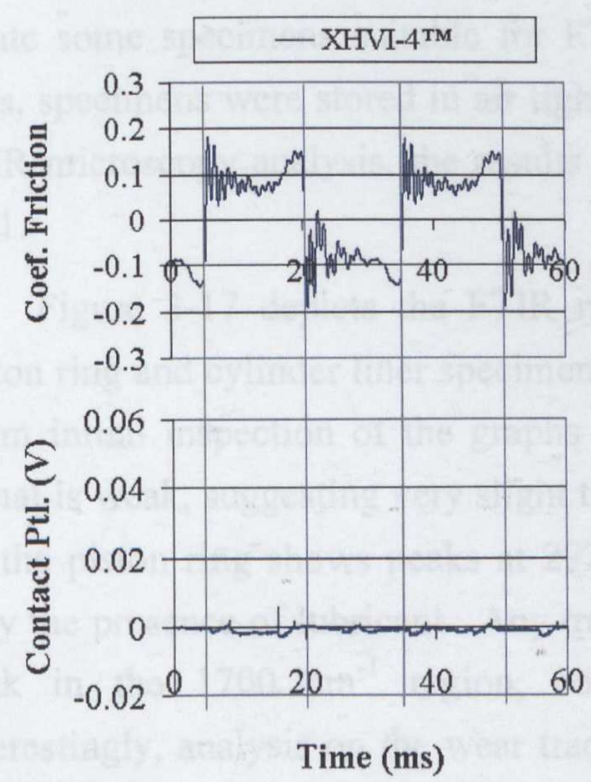

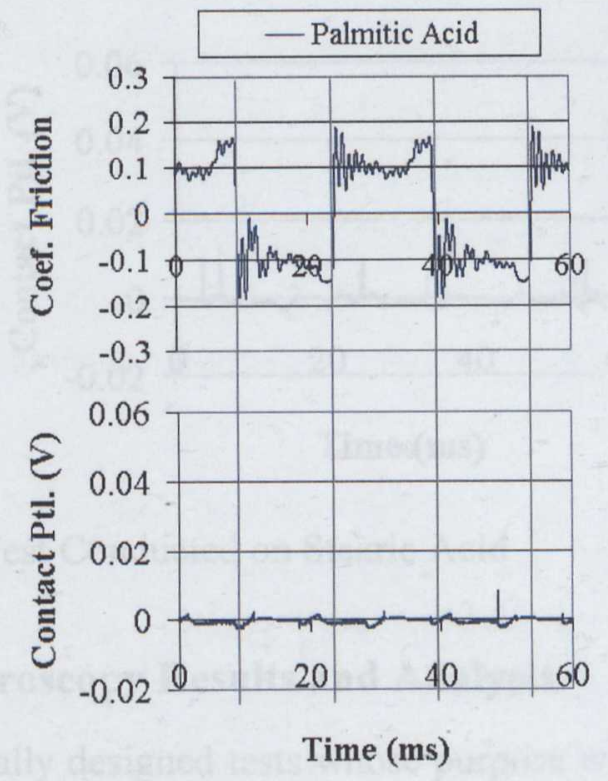

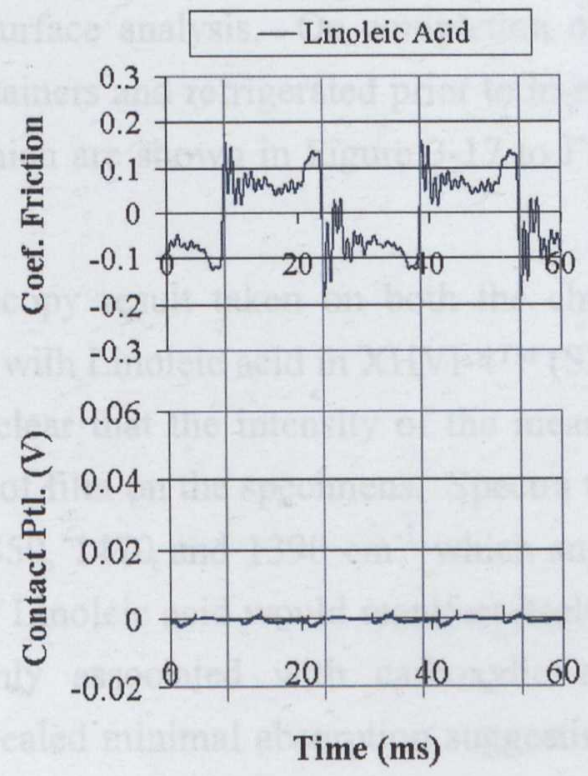

Figure 3-15 Instantaneous Friction and Contact Potential Results (Mo Ring)

Figure 3-16 depicts the contact potential traces taken from a test run conducted with a $5 \mathrm{~mm}$ stroke at $60^{\circ} \mathrm{C}, 40 \mathrm{~N}$ and $2000 \mathrm{rpm}$ for the Mo ring as described in section 3.3.4. The left-hand data set suggests the machine is running on the cusp of hydrodynamic operation, as some separation of the specimens occurs within the stroke, as represented by the rise in contact potential. However, once Stearic acid has entered the machine (right-hand data set) the contact potential measurement reads zero, typically suggestive of complete metal-to-metal contact. 


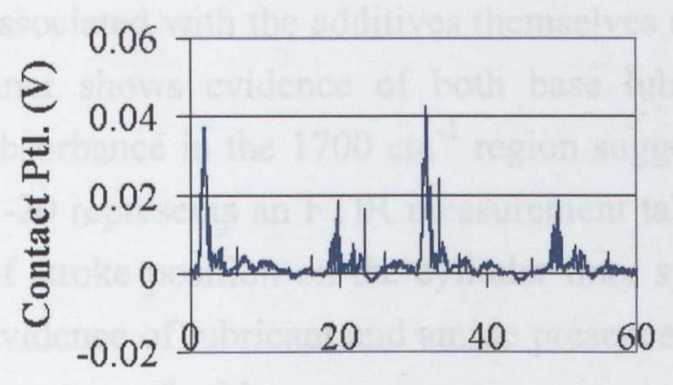

Time (ms)

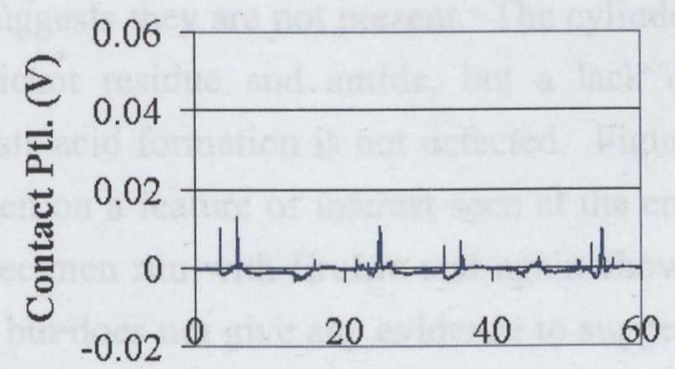

Time (ms)

Figure 3-16 Film Conductivity Test Conducted on Stearic Acid

\subsubsection{Film Creation FTIR Microscopy Results and Analysis}

Section 3.3.5 described a set of specially designed tests whose purpose was to create some specimens suitable for FTIR surface analysis. On completion of the tests, specimens were stored in air tight containers and refrigerated prior to in-house FTIR microscopy analysis, the results of which are shown in Figure 3-17 to Figure 3-21.

Figure 3-17 depicts the FTIR microscopy result taken on both the chrome piston ring and cylinder liner specimens run with Linoleic acid in XHVI-4TM (Shell). From initial inspection of the graphs it is clear that the intensity of the measured signal is weak, suggesting very slight traces of film on the specimens. Spectra taken on the piston ring shows peaks at 2920,2850, 1470 and $1390 \mathrm{~cm}^{-1}$ which suggest only the presence of lubricant. Any trace of Linoleic acid would manifest itself as a peak in the $1700 \mathrm{~cm}^{-1}$ region, commonly associated with carboxylic acids. Interestingly, analysis on the wear track revealed minimal absorption suggesting an absence on the surface of both additive and lubricant residue. On inspection of this graph it is necessary to note that the visible trough at approximately $2360 \mathrm{~cm}^{-1}$ is associated with $\mathrm{CO}_{2}$, and is a consequence of the FTIR microscopy method.

Inspection of Figure 3-18 which shows absorbance spectra for tests conducted on specimens tested with Erucylamide suggests appreciable amounts of chemicals on the surface of both chrome ring and cylinder liner. Peaks at 2940, 2860, 1460, 1380 and $720 \mathrm{~cm}^{-1}$ are thought to be associated with the base lubricant, where as peaks at $3360,3200,1660$ and $1630 \mathrm{~cm}^{-1}$ are thought to be associated with amide formation. The same frequency absorptions occur on measurements taken on both piston ring and cylinder liner, although those on the cylinder liner suggest a greater amount of lubricant and additive are present.

Tests conducted with EruLin (Figure 3-19), a blend of 1\% Erucylamide and $1 \%$ Linoleic acid in XHVI-4 ${ }^{\mathrm{TM}}$ (Shell), suggest some lubricant residue is detectable on the chrome piston ring sample, but the lack of absorbance at frequencies 
associated with the additives themselves suggests they are not present. The cylinder liner shows evidence of both base lubricant residue and amide, but a lack of absorbance in the $1700 \mathrm{~cm}^{-1}$ region suggests acid formation is not detected. Figure 3-20 represents an FTIR measurement taken on a feature of interest seen at the end of stroke position on the cylinder liner specimen run with EruLin and again shows evidence of lubricant and amide presence, but does not give any evidence to suggest presence of acid.

When Linoleic acid was run with a molybdenum piston ring, FTIR measurement detected no residue or formation on the piston ring surface (Figure 3-21). A weak absorbance was detected on the cylinder liner surfaces and was thought to be a result of lubricant residue. Again, no evidence of the acidic additive is visible.

a)

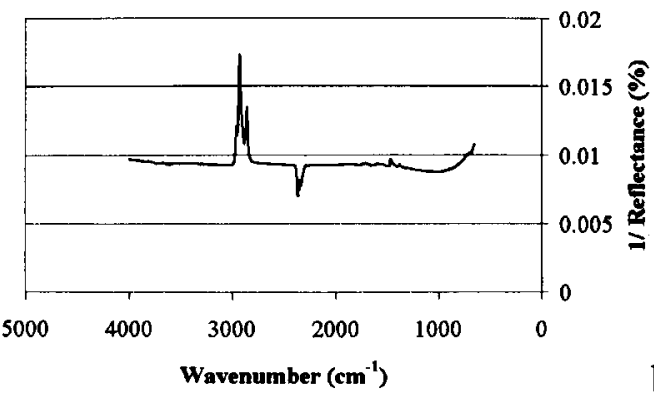

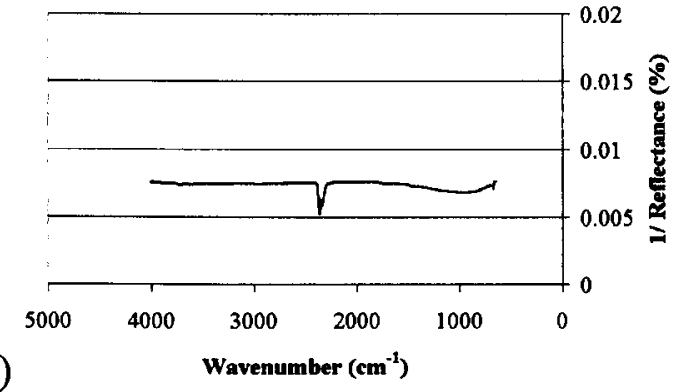

b)

Figure 3-17 FTIR Analysis of Chrome Ring (a) and Cylinder Liner (b) Run with Linoleic Acid

a)

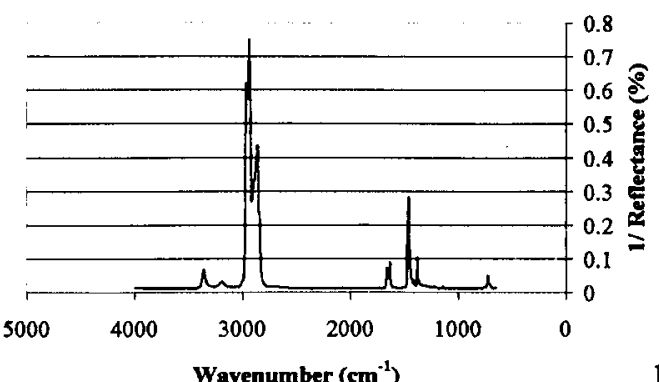

b)

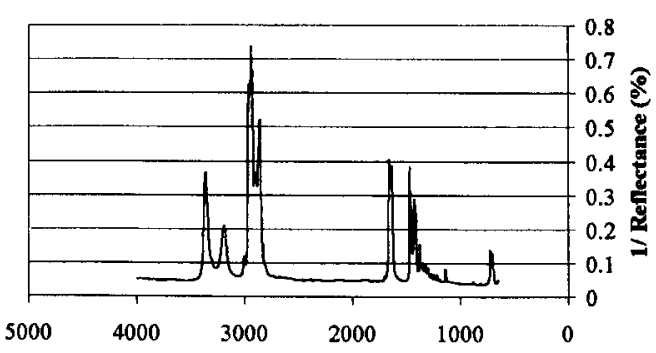

Wavenumber $\left(\mathrm{cm}^{-1}\right)$

Figure 3-18 FTIR Analysis of Chrome Ring (a) and Cylinder Liner (b) Run with Erucylamide 

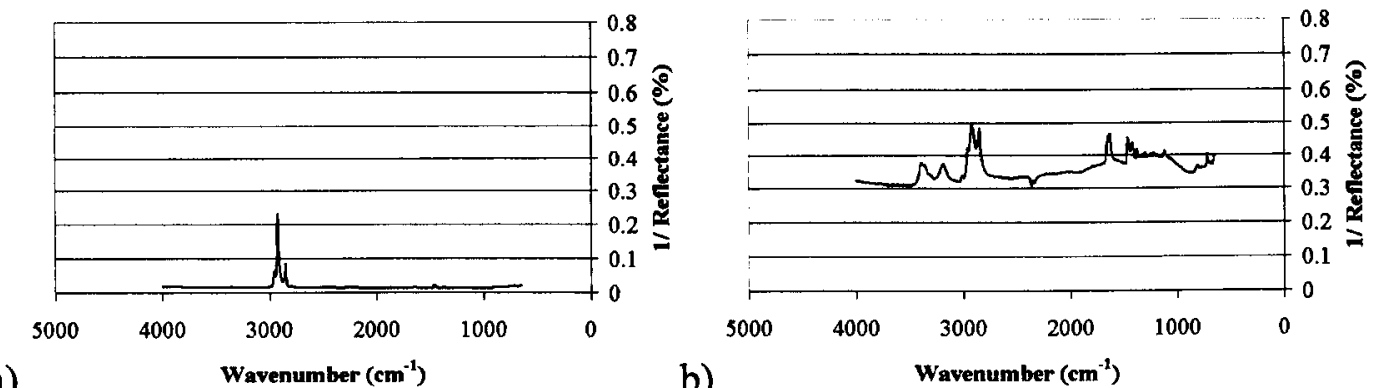

a)

b)

Wavenumber $\left(\mathrm{cm}^{-2}\right)$

Figure 3-19 FTIR Analysis of Chrome Ring (a) and Cylinder Liner (b) Run with EruLin

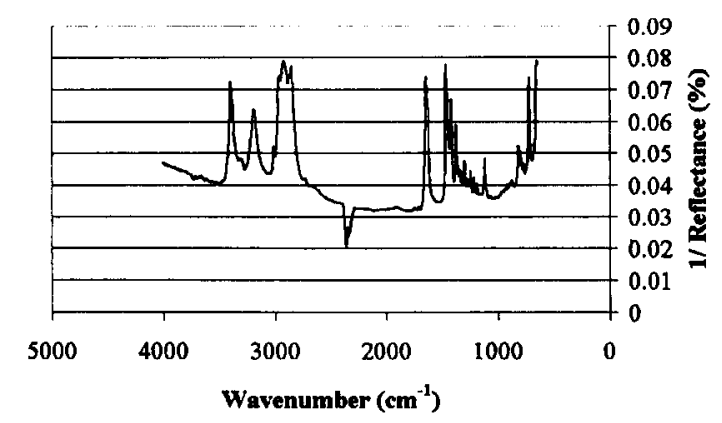

Figure 3-20 FTIR Analysis of Cylinder Liner at the End of Stroke Position Run with EruLin
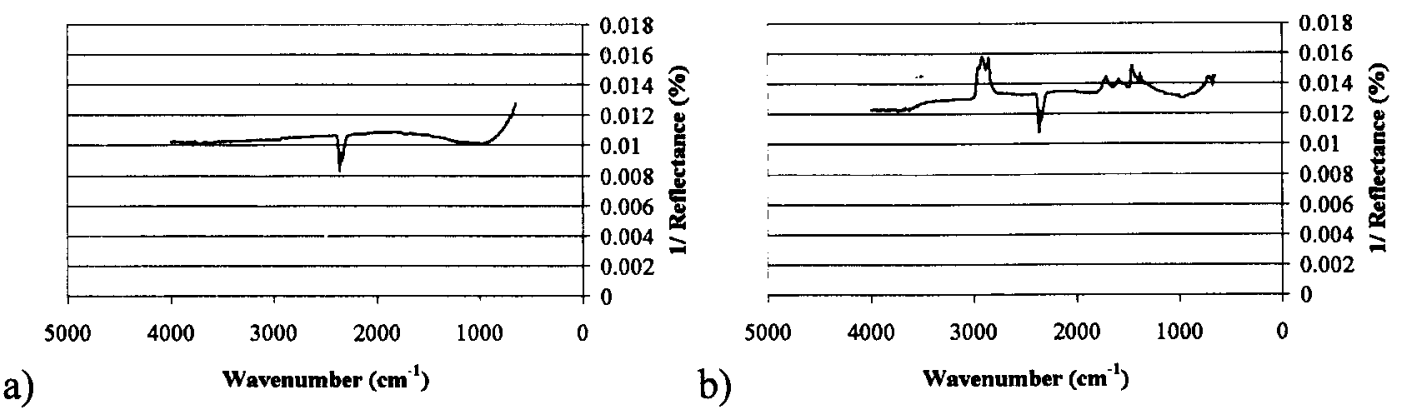

Figure 3-21 FTIR Analysis of Molybdenum Ring (a) and Cylinder Liner (b) Run with Linoleic Acid

The cylinder liner specimen which was run with a chrome coated piston ring and the $2 \mathrm{wt} \%$ Erucylamide blend was selected for in-depth surface analysis due to the interesting spectra seen on the surface when initially analysed. Figure 3-22 shows the variation in absorbance along the measurement line taken across a cylinder liner specimen. Regions of high IR absorbance are indicated by a green colour, whereas those associated with low absorbance are shown in blue. The figure clearly shows that there is a difference in the level of absorbed IR energy when 
comparing the worn area with that of the unworn. It seems there are a greater number of chemical compounds on unworn surfaces. The wire grid representation of the same surface scan is depicted in Figure 3-23, which details the detected spectra with location along the measurement line. A decrease in the level of absorbance of both base oil associated components and those of the amide additive is clearly visible between $\sim 1000$ microns and $\sim 6000$ microns in the $\mathrm{Y}$ axis, the area associated with wear. It is interesting to note that around these locations, which are associated with the end of stroke on the cylinder liner, there is a significant increase in the level of absorbed spectra indicating a greater presence of both base oil and additive.

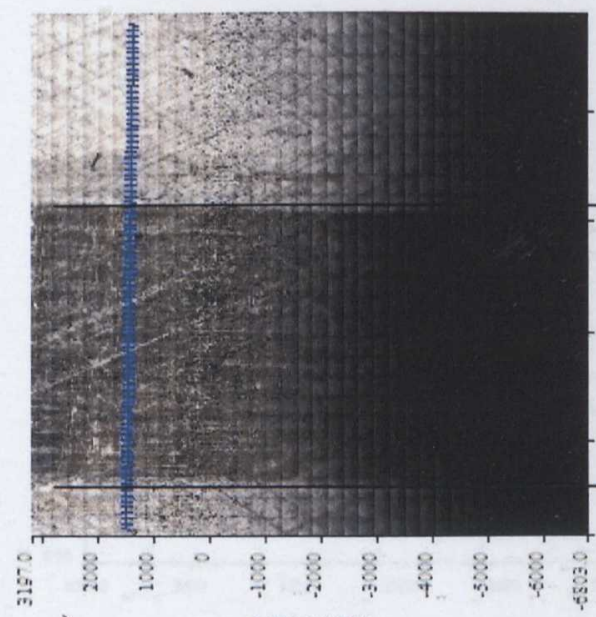

a)

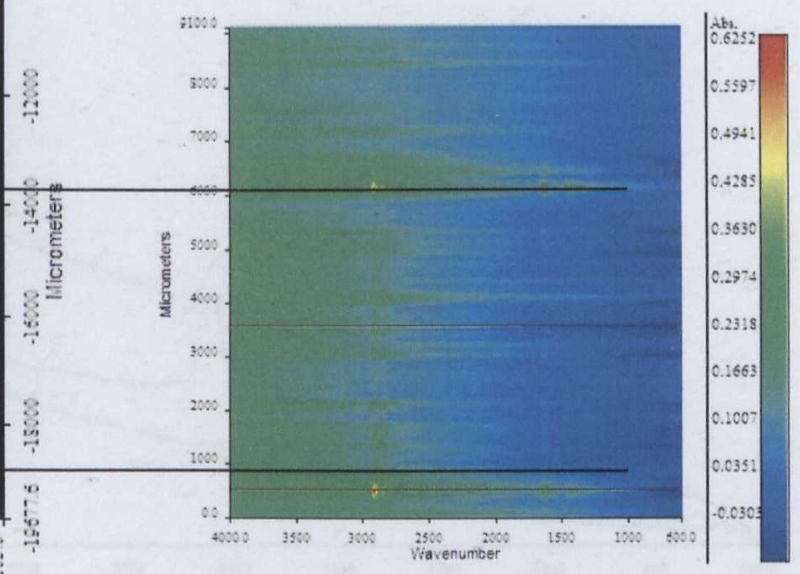

b)

Figure 3-22 Optical Image (a) and FTIR Line Scan (b) of Cylinder Liner Specimen Run with Erucylamide

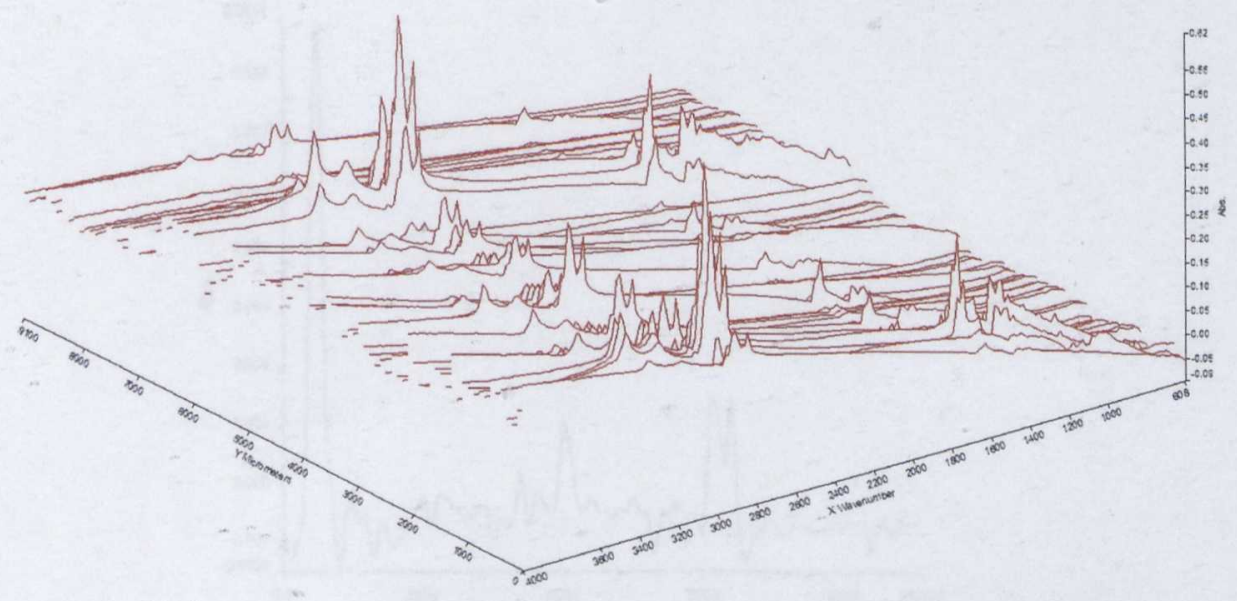

Figure 3-23 Wire Grid Projection of FTIR Scan on Cylinder Liner Specimen Run with Erucylamide 
Figure 3-24 shows the variation in intensity of the spectra at $400 \mu \mathrm{m}$ (off wear track), $4500 \mu \mathrm{m}$ (on wear track) and $8400 \mu \mathrm{m}$ (off wear track) microns along the measurement line. Although these data appear to be similar to those presented previously taken using the Nicolet microscope, these spectra are conducted at higher spectral resolution, and as such highlight some potentially interesting variations in the spectra, particularly in the $1640 \mathrm{~cm}^{-1}$ and $1560 \mathrm{~cm}^{-1}$ region. Figure $3-25$ displays this single-frequency variation in absorbance at $1560 \mathrm{~cm}^{-1}$ (relative to a baseline at $1575 \mathrm{~cm}^{-1}$ ), a region related to the $\mathrm{N}-\mathrm{H}$ bonding within the amide structure. It is clear from this figure that the concentration of the absorbance is greater off the worn area than on it, with visible absorbance peaks at the boundaries.

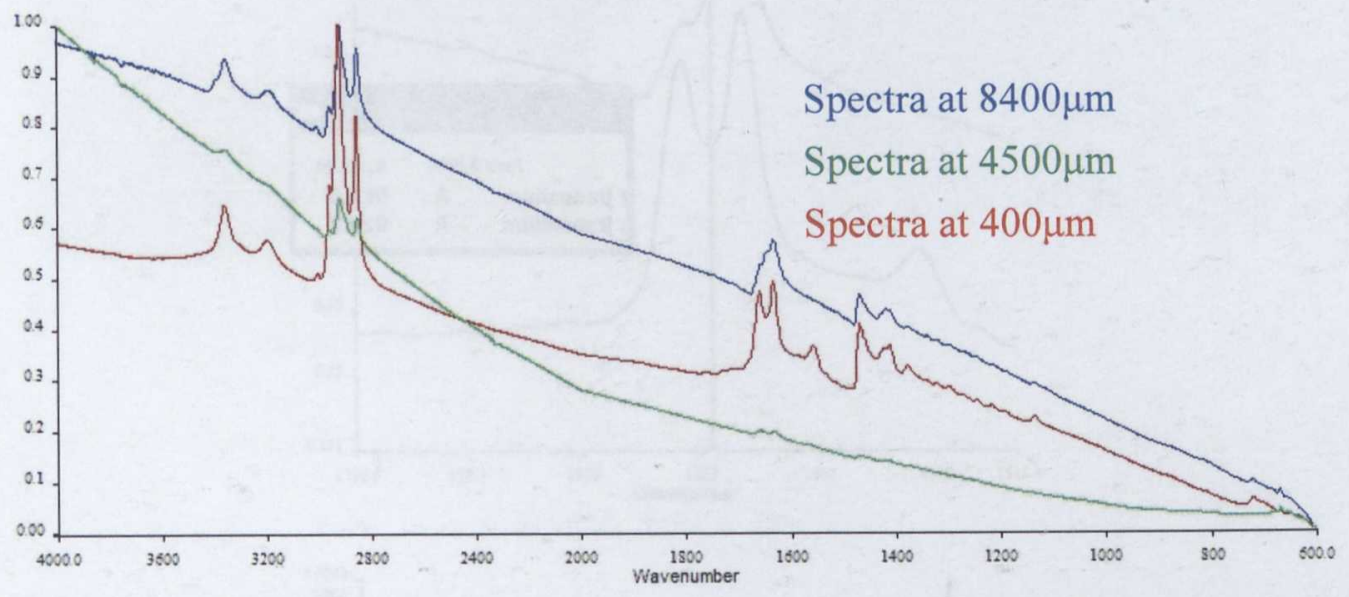

Figure 3-24 Individual FTIR Spectra at 400, 4500 and 8400 microns on Cylinder Liner Specimen Run with Erucylamide

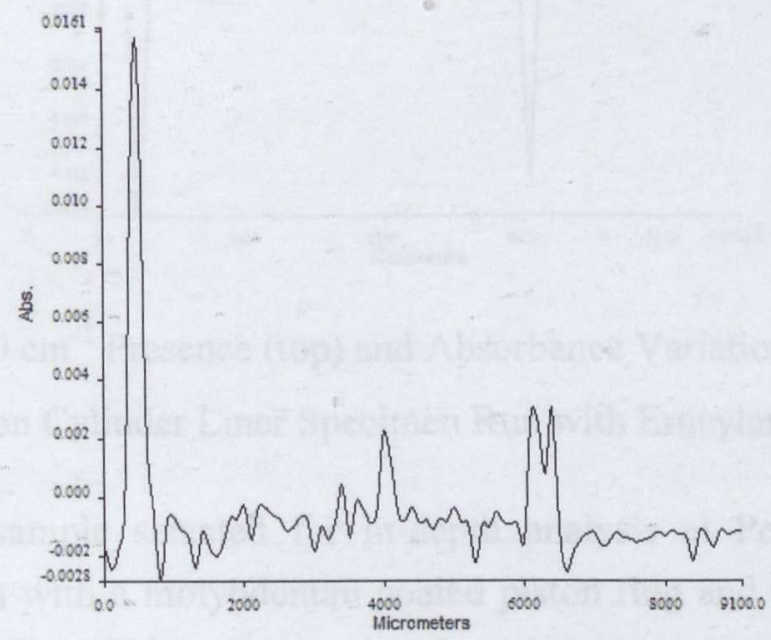

Figure 3-25 $1560 \mathrm{~cm}^{-1}$ Absorbance Variation on Cylinder Liner Specimen Run with Erucylamide 
The variation in the band associated with $1640 \mathrm{~cm}^{-1}$ is not interesting in concentration terms, but when expanded it is clear that an additional absorbance band occurs at $\sim 1645 \mathrm{~cm}^{-1}$, and is present at various points along the measurement line scan (Figure 3-26). Investigating this phenomenon further, Figure 3-26 shows how the absorbance at $1645 \mathrm{~cm}^{-1}$ varies (relative to a baseline at $1652 \mathrm{~cm}$ ) along the measurement line scan. The figure clearly shows a lack of the $1645 \mathrm{~cm}$ absorbance in the regions associated with the deposits outside the end of stroke positions, and the highest absorbance located well away from the worn region.
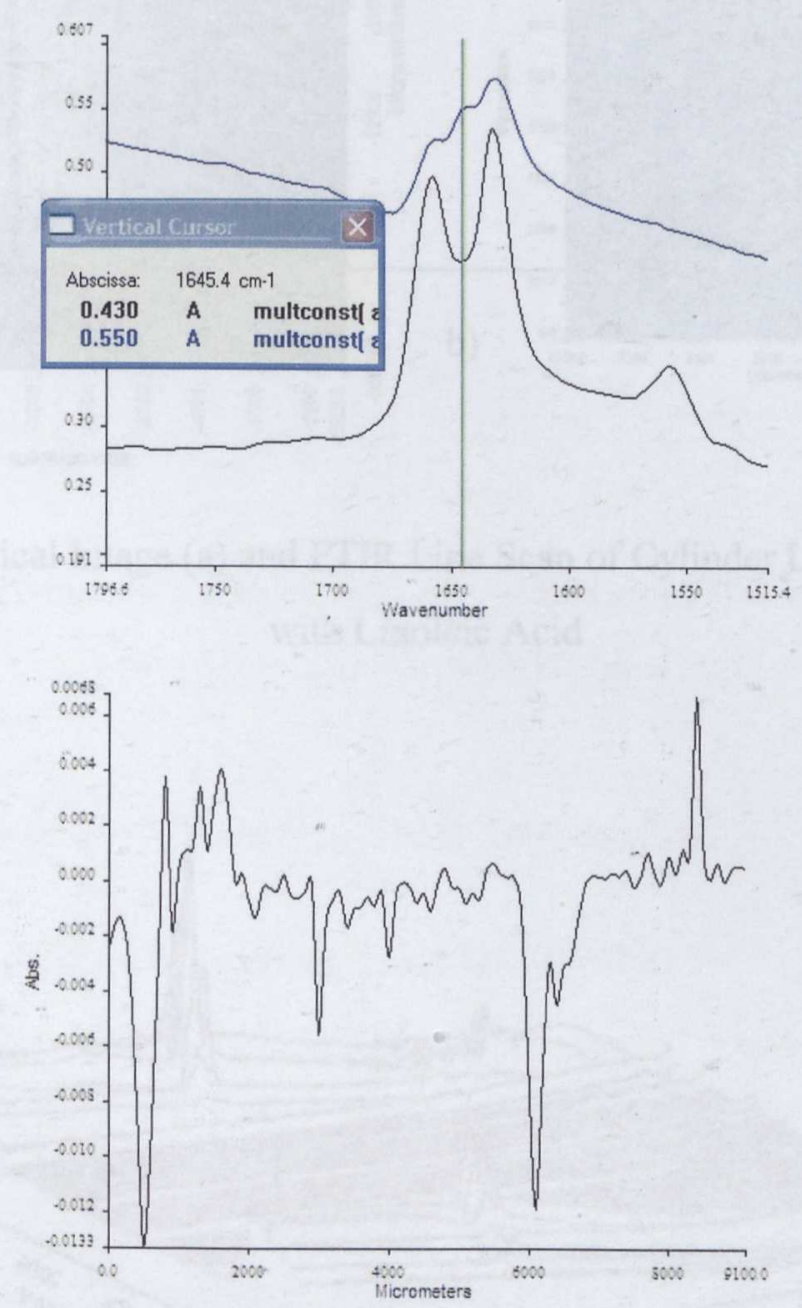

Figure 3-26 $1640 \mathrm{~cm}^{-1}$ Presence (top) and Absorbance Variation $1645 \mathrm{~cm}^{-1}$ (bottom) on Cylinder Liner Specimen Run with Erucylamide

The final sample selected for in-depth analysis at Perkin Elmer was the cylinder liner run with a molybdenum coated piston ring and with Linoleic acid as the friction modifier. This sample was selected as data associated with it showed interesting results in the friction measurement tests discussed previously. Again a series of scans were taken at 100 micron intervals across the surface of the cylinder 
liner specimen as depicted by left hand optical image featured in Figure 3-27. The right hand image shows the absorbance levels associated with each area on the measured surface, and clearly depicts a lack of any chemical within the worn area. Outside the wear track there appears to be a low level of absorption which peaks close to the boundary between worn and unworn area.

a)

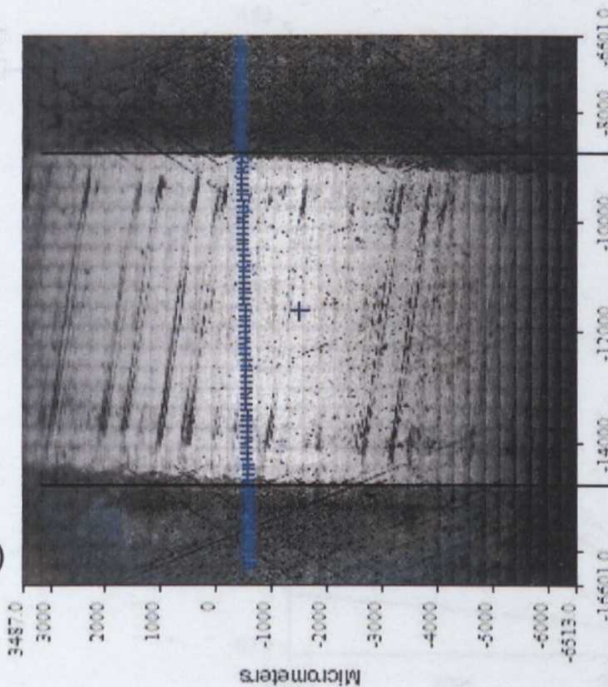

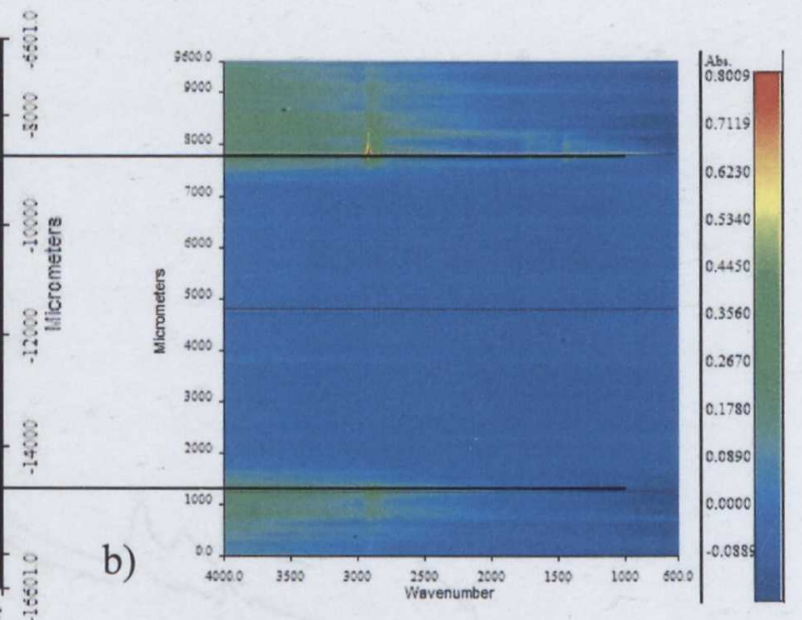

Figure 3-27 Optical Image (a) and FTIR Line Scan of Cylinder Liner Specimen Run with Linoleic Acid

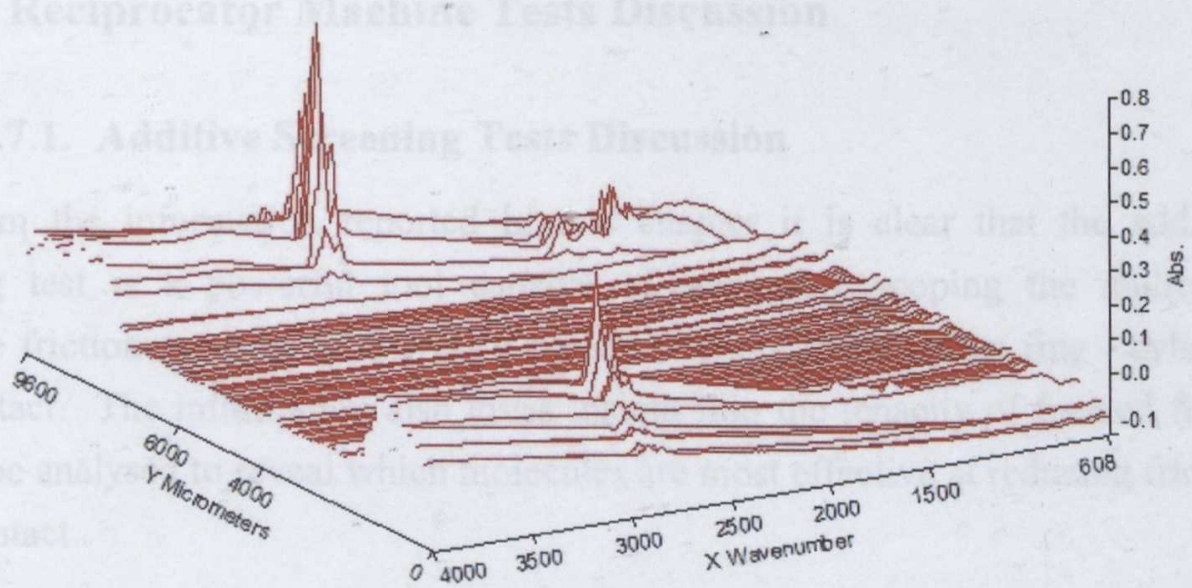

Figure 3-28 Wire Grid Projection of FTIR Scan on Cylinder Liner Specimen Run with Linoleic Acid

The wire grid projection shown in Figure 3-28 describes the chemistry of the surface further, indicating the worn area is relatively clean and shows little evidence of any chemical residue from either base oil or friction modifier. This is a 
conclusion further validated through analysis comparing worn and unworn surfaces in Figure 3-29. However, unlike the similar analysis conducted with the cylinder liner specimen run with amide additive, there appears to be little variation in the spectra where seen.

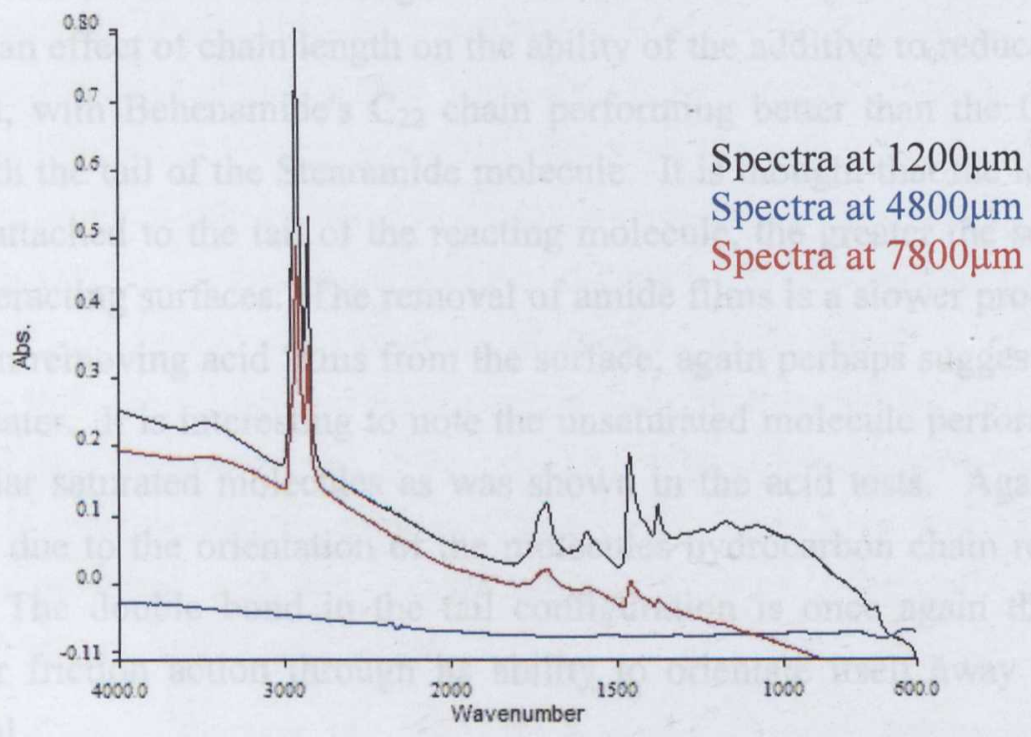

Figure 3-29 Individual FTIR Spectra at 1200, 4800 and 7800 microns on Cylinder Liner Specimen Run with Linoleic Acid

\subsection{Reciprocator Machine Tests Discussion}

\subsubsection{Additive Screening Tests Discussion}

From the information reported in this chapter it is clear that the additive screening test is a powerful tool capable of accurately scoping the ability of candidate friction modifier additives to reduce friction in the piston ring / cylinder liner contact. The information also gives insight into the tenacity of formed films, and can be analysed to reveal which molecules are most effective at reducing friction in the contact.

The chrome ring results show that the acid molecules appear to form relatively slowly on the interacting surfaces, taking around ten minutes to form a stable film. Their tenacity appears weak as they are removed remarkably quickly from the surfaces once fresh additive supply is removed from the contact. The unsaturated Linoleic acid molecule appears to perform better than the saturated molecules. As the only variable in this comparison is the saturation of the molecule it is hypothesised that the unsaturation of the Linoleic acid hydrocarbon tail allows it to 
orientate itself at an angle other than normal to the surface, as is expected to be the case with saturated molecules. This property appears to be beneficial in reducing coefficient of friction within the contact.

The amide response is clearly different to that of the acids, as all film formation occurs in approximately 1 minute from entering the contact, perhaps giving an indication as to the strength of the attachment to the surface. There appears to be an effect of chain length on the ability of the additive to reduce friction in the contact, with Behenamide's $\mathrm{C}_{22}$ chain performing better than the $\mathrm{C}_{18}$ chain associated with the tail of the Stearamide molecule. It is thought that the longer the chain length attached to the tail of the reacting molecule, the greater the separation of the two interacting surfaces. The removal of amide films is a slower process than that seen when removing acid films from the surface, again perhaps suggesting their tenacity is greater. It is interesting to note the unsaturated molecule performs better than the similar saturated molecules as was shown in the acid tests. Again this is thought to be due to the orientation of the molecules hydrocarbon chain relative to the surface. The double bond in the tail configuration is once again thought to provide lower friction action through its ability to orientate itself away from the surface normal.

Both the performance of Tristearin and the Acetic Acid Alkyl Esters was poor. It is thought that the $\mathrm{C}=\mathrm{O}$ configuration and ester act to block the reactive ends action on the surface. Where film is formed, the clumsy configuration of the molecule is such that its orientation on the surface is far from normal. It suggests that as well as a long chain length, the additive film needs to be capable of forming films well orientated to the surface.

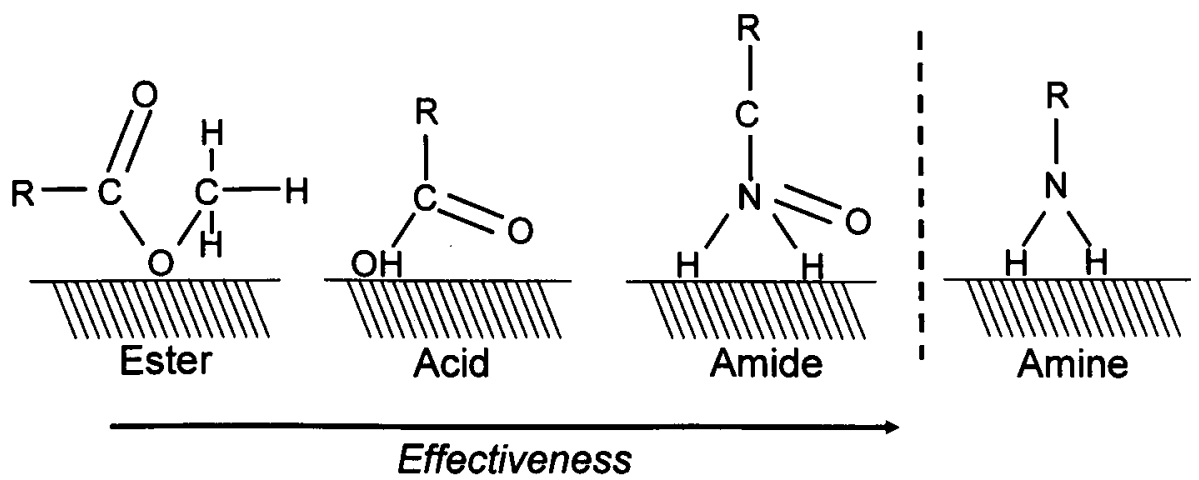

Figure 3-30 Influence of Molecular Orientation on Additive Performance

The information gained regarding the effectiveness of the additives at reducing friction in the contact is shown schematically in Figure 3-30. From the analysis of 
Ester, Acid and Amide films it is possible to hypothesise that a more effective molecular compound might be that of an amine, although this theory remains untested. The hypothesis that an amine molecule would be more effective at friction reduction than that of ester, acid and amide stems from the culmination of results suggesting that the presence of a strong polar head $\left(\mathrm{NH}_{2}\right)$ and lack of $\mathrm{C}=\mathrm{O}$ feature may be beneficial. In fact, on consideration of all information gained from the preceding results it is possible to hypothesise the 'ultimate' friction modifier additive as one whose species is amine, has a long chain length $\left(\mathrm{C}_{22}\right.$ or greater), and whose hydrocarbon tail is polyunsaturated. However, preceding chapters have told us that additive which is administered to the engine via the fuel will find its way into the sump lubricant. Given this knowledge it is necessary to acknowledge that having polyunsaturated, or indeed unsaturated, molecules in an engine's lubricant is undesirable as they are prone to cross linking, and as such would be likely to form sludge, degrading the performance of the oil. However, it is also necessary to note that should the molecules employed be saturated, there should be no degrading effect on the engine lubricant, and their performance is thought to be only marginally poorer than their unsaturated equivalents.

Direct analysis of the raw data taken from the TE-77 gives another dimension to the investigation into ' $\mathrm{CHON}$ ' friction modifiers through giving information relating to the action, or mechanism of friction reduction of the additives. Consideration of the instantaneous friction force and contact potential data has been analysed to give this information.

The acid, amide and triglyceride families appear to all form surface films capable of reducing the friction of the sliding contact between piston ring and cylinder liner. Although the ester formation appears to have some friction reducing properties it is the only additive whose contact potential data suggests it didn't form an appreciable surface film. Fatty acid molecules seem to form surface films which almost completely isolate the interacting surfaces from one another, a quality not shared by the other chemical species under investigation. It is hypothesised that the molecules form dense surface films with poor electrical conductivity in a well orientated manner, essentially interposing a molecular sheet between the sliding piston ring and liner. Of the fatty acid molecules under investigation it appears the films formed by the application of Linoleic acid, the polyunsaturated variant of Stearic acid, are slightly weaker as signs of contact are evident at stroke reversal. This may be the result of the orientation of the additive to the surface, which is likely to be away from the normal due to the unsaturated nature of its hydrocarbon tail. However, it is also hypothesised that it is this non-normality with the surface that leads to lower friction sliding in the mid-stroke region. 
The formation of amide films is not unlike that of acid films, although there are subtle differences which set them apart. Typically the films formed through application of amide appear to be less complete than those formed through application of acid based friction modifier. In general, the amide film formation is incomplete in that there appears to be a lack of film at the stroke reversal points. However, it is acknowledged that it may also be possible that the film coverage is complete, but arduous conditions at the reversal points cause local film removal. This is a theory which requires further investigation to validate. It is interesting to note that there is a large difference between the Stearamide $\left(\mathrm{C}_{18}\right)$ and Behenamide $\left(\mathrm{C}_{22}\right)$ contact potential data, again suggesting that longer hydrocarbon tails form more successful friction modifier films. The action of the Erucylamide molecule is of key interest, as it gives extremely low friction action at the mid-stroke, and greater contact insulation than other amide additives. It is hypothesised that this exceptional performance as a friction modifier comes from the combination of what seem to be two desirable properties, a long chain length and unsaturation.

It is no surprise that the films formed through application of triglyceride and ester molecules look to be poor, as the previous analysis of the resulting coefficients of friction showed the two species to be the weakest performing friction modifier additives. Although the contact potential measurement associated with the action of Tristearin looks to be quite complete, the low value of electrical insulation perhaps suggests a very thin, or incomplete, film is formed. Coupled with the instantaneous friction data, it is clear that any film formed has little effect on the frictional characteristics of the contact.

Of key importance in this chapter is the understanding that acid based friction modifier additives appear to form complete surface films, even at stroke reversal points, and that amide films form potentially lower friction films in the mid-stroke, but are not effective at stroke reversal locations. It was on consideration of these facts that the blend of $1 \mathrm{wt} \%$ Linoleic acid and $1 \mathrm{wt} \%$ Erucylamide, termed EruLin in this research, was conceived. It was thought that the two species could complement each other by achieving a lower coefficient of friction for the contact than either of the additives on their own.

It was hoped that the combination of an unsaturated acid and amide would lead to lower friction sliding for the components in the test as it appears both additives work in different ways. It was thought that the amide would dominate the film formation due to its rapid reaction characteristic, only leaving exposed metal at the stroke reversal points where it is either incapable of forming, or is easily removed. At these sites of exposed metal it was thought that acid film formation would occur completing the interposed layer and fully separating interacting surfaces (Figure 
3-31). Given this formation system it was hoped the contact would benefit from the low frictional characteristics of the unsaturated amide film in the mid-stroke and the unsaturated acid formation at stroke reversal.

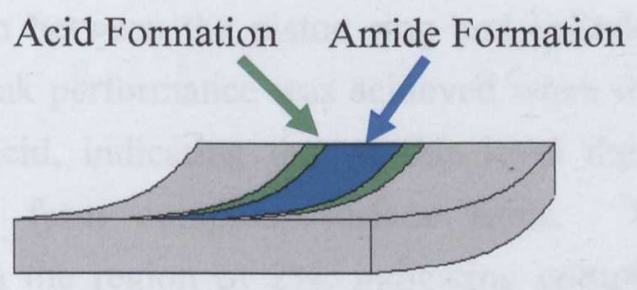

Figure 3-31 Hypothesised Formation of Additive Films

The coefficient of friction data suggests a reduction of $50 \%$ through utilisation of the blend of additives, $3 \%$ less than that achievable with Linoleic acid alone which may indicate the blend creates less effective friction modifier films. However, the data presented in Figure 3-12, detailing the instantaneous friction and contact potential measurements for the blend, suggests a more effective friction modifier film formation. On consideration of this fact it is possible to interject a hypothetical explanation for this phenomenon. It is necessary to remind the reader at this point that the calculation of reduction in coefficient of friction is conducted through comparison of the last baseline point (30 mins) and the last friction modifier point (90 mins), and as such does not consider the final coefficient of friction as a value, only as a constituent of the percentage reduction. This method of comparison makes no consideration that the effectiveness of the film may be dependant on the final coefficient of friction. It is clear on inspection of Table 3-5 that the final coefficient of friction for the additive blend is lower than that associated with either of the constituent additives alone. This perhaps suggests that in some cases consideration of the final coefficient of friction as a stand-alone value is a more proficient indicator of an additive's performance. This outcome suggests the blending of additives in an informed manner can lead to the creation of more effective friction modifier films. However, it is necessary to state at this point that it is acknowledged that the EruLin additive package formulated in this work is a direct product of this test alone, and as such presents itself as an additive package specifically tailored to the given lubrication conditions in the test conducted.

\subsubsection{Additive Concentration Tests Discussion}

Tests were conducted to investigate the influence of additive concentration on friction within the piston ring / cylinder liner contact. Blends containing different levels of Linoleic acid in XHVI-4 ${ }^{\mathrm{TM}}$ (Shell) were tested in the TE-77 machine. 
Results indicate that a similar performance is achieved through application of additive concentrations in the range of $0.5-4 \mathrm{wt} \%$ by weight. Once additive concentration is reduced below the $0.5 \mathrm{wt} \%$ level the performance of the additive is poor. At this concentration it is thought that there was an insufficient number of additive molecules for complete surface coverage of the film resulting in a greater proportion of interaction between the piston ring and cylinder liner, thus creating greater friction. The peak performance was achieved when the base oil was doped with $2 \mathrm{wt} \%$ Linoleic acid, indicating that at this level there are ample friction modifier molecules to form complete surface films. With the plateau of effectiveness reached in the region of $2 \%$, indicating complete surface coverage, there is no reason that the $4 \%$ solution should perform any better. At the $4 \%$ treatment level one could speculate that a micelle, or aggregate of additive molecules, could form in the blend. A typical micelle would be expected to form an aggregate with the reactive head ends of the molecule in contact with the surrounding lubricant, sequestering the molecule tails in the micelle centre. This may have resulted in the micellization of globular shaped aggregates of additive capable of affect the performance of the system.

The effect of time on the reduction of coefficient of friction is also clearly evident on inspection of Figure 3-13. Looking at the points associated with an additive doping level of $0.25 \mathrm{wt} \%$ it is clear that a film has formed in the contact at the 40 minute (or 10 minutes of additive) mark and continues to form until approximately 60 minutes where no further change occurs, indicating that all available molecules have been employed on the surface after 20 minutes of additive, and that no further film formation occurs. However, the increased ability for the formulations with additive concentrations in excess of $0.25 \mathrm{wt} \%$ to reduce friction with time suggests the formation of multi-layer, or more likely, more complete films.

It is worth noting here, that it is acknowledged that the performance of the additive with relation to concentration is likely to depend heavily on the system within which it is employed. As discussed earlier, the polar nature of the films means they will form on any exposed metal. Should the amount of exposed metal be large, a large concentration of additives may be required to ensure adequate friction modifier is available for formation within the contact area.

It is necessary to remember that for the purposes of this research, the friction modifier is to be administered to the engine via the fuel, and that as such it is not the case that a finite amount of molecules are available for friction reduction purposes. Through application of the friction modifier with the fuel as a carrier, more and more additive will enter the engine with every pulse of the fuel injector, administering constantly replenished supplies of friction modifier to the engine surfaces. 


\subsubsection{Piston Ring Material Friction Discussion}

The in-house standardised additive screening test was conducted with a molybdenum coated piston ring running against cast iron liner. Tests were conducted using blends containing $2 \mathrm{wt} \%$ Stearic acid, Palmitic acid and Linoleic acid in XHVI-4 ${ }^{\mathrm{TM}}$ (Shell).

The results displayed in this section clearly highlight the influence piston ring selection has on the ability of the additives to reduce friction in the contact, and cause us to ask many questions.

It is evident that Stearic acid performed better than Palmitic acid when considering the initial reduction in coefficient of friction, this is perhaps due to the slightly longer chain length associated with the Stearic acid molecule. The performance of the polyunsaturated molecule, Linoleic acid, is of considerable interest. The action of the molecule is clearly very different to that of its saturated variant, Stearic acid. It seems film formation is slow, but effective, as it continues to reduce friction within the contact throughout its duration in the machine. Interestingly it seems the tests conducted with the molybdenum coated piston ring are more likely to return to the baseline coefficient of friction than tests conducted with the chrome coated ring.

The result recorded in Figure 3-16 depicting the instantaneous friction force and contact potential data is of real interest when trying to understand the difference between the action of the additives when employed with a chrome ring, and those seen when running on a molybdenum ring. The zero contact potential measured after 60 seconds when running with Stearic acid and molybdenum ring tells us something has happened in the system which makes the contact better at conducting electricity.

The chrome and molybdenum coated rings have extremely different surface finishes, molybdenum being considerably rougher than that of chrome. As such it is possible that the effects seen are caused by the rough abrasive nature of the flame sprayed molybdenum ring. The chrome and molybdenum rings also offer surfaces with different compositions to the reactive additive, which could possibly influence the type of reaction taking place between additive molecule and metal substrate.

To fully investigate the reasons for the dramatic difference in the results associated with tests conducted with a chrome piston ring section and those conducted with a molybdenum ring a great deal of specially designed further testing is required. Variation in the surface roughness and surface composition need to be considered in isolation to decouple the two possible influences. As such, and within the bounds of this investigation, the results obtained to date serve merely to highlight 
an interesting phenomenon, whose explanation lies outside the scope of the current research project. However, this is an area which has been highlighted for further work, and is detailed in Conclusions \& Recommendations for Future Work later on in this thesis.

\subsection{Film Creation FTIR Microscopy Discussion}

The relatively weak signals measured through FTIR analysis on the component surfaces make conclusive discussion difficult. However, some qualitative discussion is made possible through analysis of the data as a whole.

Analysis of samples subject to operation with Linoleic acid revealed that both piston ring and cylinder liner surfaces had next to no chemical presence, and that even residue from the base oil was scarce. This is a surprising result as the preparation of the surfaces prior to analysis only consisted of flowing over dry compressed air. As this type of surface 'cleaning' is relatively non-intrusive it would be expected that some lubricant residue be present on the surface where analysed.

Tests conducted with Erucylamide reveal a completely different story, showing identifiable spectra known to be associated with both the base oil and amide additive. Their presence is detected on both piston ring and cylinder liner surfaces suggesting residue remained on both interacting surfaces.

The test conducted with a blend of both Erucylamide and Linoleic acid exhibited a relatively intermediate strength absorbance spectra on the cylinder liner, and weak absorbance on the chrome ring suggesting residue is present in greater quantities on the cylinder liner surface. This is not surprising as the surface of the cylinder liner is engineered to retain lubricant. The presence of identifiable spectra in the EruLin test is thought to be solely due to the amide additive as no peaks associated with carboxylic acid was seen. It is worth noting at this point that the melting points of the additives are likely to have a pronounced effect on the strength of signal obtained, as Linoleic acid is liquid at room temperature and Erucylamide is solid. It is thought that cleaning with compressed air is more likely to remove liquid particles than solid, which may have formed an attachment to the surface.

Although the spectra obtained from the FTIR analysis of the component surfaces indicated the presence of amide additive on surfaces subject to Erucylamide, the strength and resolution of the signal obtained make any judgement of the chemical state of surface films impossible. However, the presence of films investigated through FTIR analyses is an interesting observation in its own right. 
Samples sent to Perkin Elmer for in-depth surface analysis revealed some interesting information. It is clear that the concentrations of additive on and off the wear track differ. On the cylinder liner specimen run with Erucylamide the reduced concentration of film on the wear track is thought to be a direct result of film removal or thinning by the piston ring. The highest concentration of additive measured outside the end stroke regions indicates amide removed from the contact by the reciprocating piston ring probably forms at the boundaries of its operation. Of key interest is the variation in spectra taken at different points on the surface, which suggests not only variation in intensity, but variation in the spectra themselves. These spectral variations could be related to variations in the chemical structure across the surface. The variation from $1650 \mathrm{~cm}^{-1}$ to $1645 \mathrm{~cm}^{-1}$ appears significant as its intensity on the surface falls at the stroke boundaries. It is at these locations the $1560 \mathrm{~cm}^{-1}$ absorbance is elevated suggesting some chemical state change as a result of surface condition. This phenomenon might explain the lack of effective amide films seen at stroke reversal points as measured through contact potential methods, and discussed in earlier sections.

In-depth analysis on the cylinder liner subject to Linoleic acid and run with a molybdenum ring again gives greater resolution as to the action of the additive itself. It is clear that in the working section of the cylinder liner specimen there is a lack of any chemical residue, however, outside this area weak concentrations of residue are seen. There may be several reasons for this, the first and most likely being that the worn surface is less capable of trapping lubricant residue due to its polished nature, and that outside the area some lubricant residue is ingrained in the rough liner hone. It is important to note that detectable spectra obtained do not indicate the presence of carboxylic acid, and looks to only be a result of the base oil absorbance. As with the cylinder liner subjected to Erucylamide, the full surface scan depicts greater intensity films at the boundaries of the working area suggesting additive removed by the reciprocating ring reforms at these locations.

Ultimately the analysis leads us to believe that the molybdenum ring / Linoleic acid system is more straightforward than that of the chrome ring / Erucylamide system, where there is some evidence of attachment or chemical state variation.

It is acknowledged by the author that the FTIR analysis of the cylinder liner surfaces leaves many questions unanswered with regards to the formation and mechanism of friction reduction of the additives under investigation, questions which are looked to be answered in following chapters. As such this section serves to summarise analyses which were performed to scope the films responsible for friction reduction in order to guide further, more focused and specifically designed investigation. 


\subsection{Summary}

This chapter contains a description of a tribometer based investigation into the performance of novel organic friction modifiers. A bespoke apparatus configuration was used to both screen candidate additives performance and investigate the mechanisms underpinning their action as friction modifiers. In addition some surface analysis was conducted on selected specimens used in the tests. Based on consideration of all the information presented in the preceding chapter, the following summary can be made.

- The best performing friction modifier additive was shown to be Linoleic acid, which reduced friction in the cylinder liner / piston ring contact $53 \%$.

- Evidence suggests that additives with long hydrocarbon tails are better than those with short tails at reducing friction in the contact.

- Evidence suggests that polyunsaturated molecules reduce friction more effectively than saturated molecules.

- The performance of triglyceride and ester molecules is thought to be poor due to configuration of their molecules which hinders effective film formation.

- Research conducted allows the hypothesis that the most effective friction modifier for the specific contact investigated would be a long, polyunsaturated amine molecule.

- Acid based friction modifiers appear to form complete surface films, and can form films in conditions where amide based friction modifiers cannot.

- It is thought that films formed through application of amide based friction modifier can reduce friction in the mid-stroke region of the reciprocator stroke more effectively than fatty acid friction modifier.

- Research conducted supports the hypothesis that it is possible to employ two chemicals in an additive package, whose purpose is united, to reduce friction in the contact. Through blending of friction modifier chemicals, more effective friction modifier films can be formed.

- Analysis of the effects of additive concentration revealed that for the chemical tested (Linoleic acid), and in the specific test described previously, the additive concentration for optimum friction reduction is 
$2 \mathrm{wt} \%$. Below $0.5 \mathrm{wt} \%$ the performance of the friction modifier was shown to be poor.

- The material and surface topography of the piston ring section has a great influence on the performance of the friction modifier additives. Tests conducted in this chapter reveal more effective friction modification with a chrome coated piston ring rather than a flame sprayed molybdenum piston ring.

- FTIR analysis of the specimen surfaces show traces of lubricant residue. No evidence of fatty acid friction modifier was detected, although the presence of amide was shown to exist where it was employed.

- Large deposits of unreacted additive were seen at the boundaries of stroke reversal, and there was some evidence to suggest a change in the chemical state of the amide additive at these points.

- There was no conclusive evidence of chemisorbed additive films on specimen surfaces, although the presence of surface chemistry detected through FTIR analysis suggests surface films exist. 


\section{Mini Traction Machine Investigation of Novel Friction Modifier Additives}

In Chapter 3, a set of experiments was conducted using a high frequency reciprocating tribometer to screen the performance of prospective novel friction modifier additives in the boundary lubrication regime. These tests simulated the arduous conditions of the $1^{\text {st }}$ compression ring in the ring zone of a firing engine. Having selected the additives most effective at reducing boundary friction, and quantified the benefits achievable through chemical compound synergism, it was deemed necessary to investigate the performance across the full spectrum of lubrication regimes that occurs in the normal operation of the piston assembly. The piston assembly passes through the boundary, mixed, elastohydrodynamic and fluid film lubrication regimes with every stroke, and that the proportion of the engine cycle spent under the boundary lubrication regime is small. In addition, as the velocity of the piston assembly tends towards zero when entering the boundary lubrication regime at the top and bottom dead centres, the overall impact on energy saved through implementation of a friction modifier at these locations is limited. A friction modifier whose capability extends to reducing friction in the mixed lubrication regime would be much more beneficial to energy conservation.

In this chapter, a series of experiments used a PCS Mini Traction Machine for three main reasons. First, the data from the machine when operating in the boundary regime might provide a basis for a trend comparison of data taken using the Plint TE-77 High Frequency Reciprocator Tribometer. Second, investigating the performance of the additive across the mixed lubrication regime is necessary when hypothesising its effectiveness in the engine environment. Third, the machine provides the perfect opportunity to create some additive films on samples suitable for more sophisticated surface analysis than the piston ring and cylinder liner samples in Chapter 3.

\subsection{The Mini Traction Machine and Test Specimens}

The PCS Mini Traction Machine (MTM) was used to investigate novel friction modifier additives, Figure 4-1. The MTM is a highly sophisticated ball on disc, computer controlled tribometer whose method of precision traction measurement provides fully automated mapping of lubricants and other fluids. The MTM machine simulates lubrication regimes in non conformal contacts such as those found in cams, valve trains and gears, for example. During a typical test, an $18 \mathrm{~mm}$ 
diameter steel ball is loaded against a $45 \mathrm{~mm}$ diameter steel disc, each independently driven to create a user defined slide/roll ratio. The contact is immersed in the test lubricant under fully flooded running conditions. The stainless steel lubricant reservoir is electrically heated via computer controlled cartridge heaters, and can be rapidly cooled by an external refrigeration circuit. Prior to a test the reservoir is covered by a stainless steel lid, the lid then covered by an insulating PTFE jacket to ensure both thermal stability and safety are achieved. Given the command, the system steps through a number of user defined loads, speeds, slide/roll ratios and temperatures whilst mapping the traction coefficient in the contact.

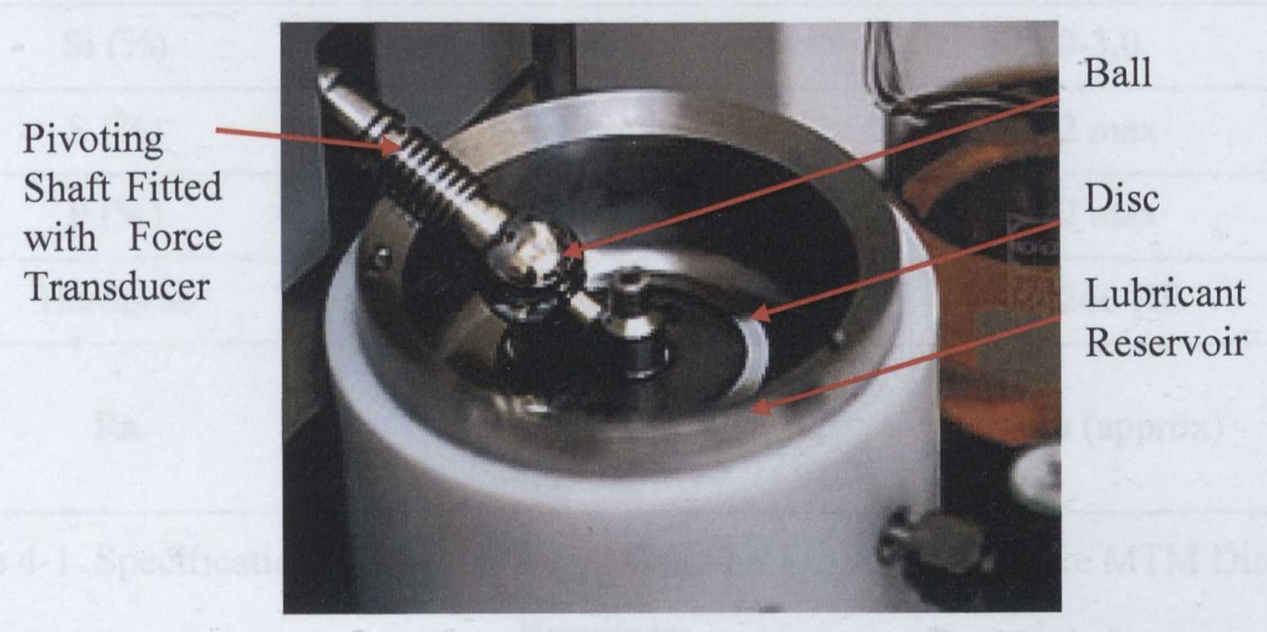

Figure 4-1 PCS Mini Traction Machine

\subsubsection{Mini Traction Machine Components}

The AISI 52100 bearing steel ball is mounted on a pivoting shaft fitted with a high sensitivity force transducer, to provide precise measurement of traction. The ball speed is controlled by a DC servo motor which allows precise control, even at very low speeds. Similarly the disc is mounted on a DC motor driven vertical shaft for precision control.

It was shown in Chapter 3 that the substrate material composition to which the friction modifier additive is exposed may have a pronounced effect on film formation. For this reason it was decided to use bespoke discs in this set of tests composed of grey cast iron material closely matched to a Ricardo Hydra cylinder liner, Table 4-1.

The Ricardo Hydra cylinder liner is finished with a $45^{\circ}-65^{\circ}$ crosshatch plateau hone, a finish extremely difficult to replicate on a flat disc. It was concluded that any attempt at replication of the surface topography would result in non-uniformity of the disc surfaces, increasing the risk of diverse experimental data with poor 
repeatability. Instead the bespoke grey cast iron discs were given a polished finish with an approximate $\mathrm{Ra}$ of 0.01 microns, the typical surface finish applied to standard specimens. This polished surface finish also aided the analysis of surface films post-test.

\begin{tabular}{ccc}
\hline Element & $\begin{array}{c}\text { Hydra Gasoline Engine } \\
\text { Cylinder Liner }\end{array}$ & MTM Disc Specimen \\
\hline $\mathrm{C}(\%)$ & $3.0-3.5$ & $3.0-3.5$ \\
\hline $\mathrm{Mn}(\%)$ & $0.6-1.2$ & $0.45-0.65$ \\
\hline $\mathrm{Si}(\%)$ & $1.8-3$ & $2.0-3.0$ \\
\hline $\mathrm{S}(\%)$ & $0.12 \mathrm{max}$ & $0.12 \max$ \\
\hline $\mathrm{P}(\%)$ & $0.4-1.0$ & $0.12 \max$ \\
\hline $\mathrm{Hardness}$ & $230-300 \mathrm{BHN}$ & $180-240 \mathrm{HB}$ \\
\hline $\mathrm{Ra}$ & $1.57 \mu \mathrm{m}$ (new) & $0.01 \mu \mathrm{m}$ (approx) \\
\hline
\end{tabular}

Table 4-1 Specification of Ricardo Hydra Cylinder Liner and Bespoke MTM Disc Specimen

\subsection{Post Test Specimen Analyses}

To understand the physical situation on a surface a variety of information is required: the physical topography; the chemical composition; the chemical structure; the atomic structure; the electronic state and a detailed description of bonding of molecules at the surface (Vickerman, 1997). However, to solve specific problems not all these aspects must be known. For this research a portfolio of surface analysis techniques was selected. Profilometry was selected to give information regarding the surface topography, scanning electron microscopy and energy dispersive X-ray techniques to give information regarding the wear process resulting in the specimen surface topography, X-ray photon spectroscopy to give detailed information regarding chemical species and their bonds near the surface of the specimen, and Fourier Transform Infra-Red spectroscopy to give information regarding chemical compounds and their state on the surface. Figure 4-2 shows the engineering surface and various analysis techniques which can be used to give information detailing the situation. All surface analyses were conducted by skilled analysts in-house at Shell Global Solutions (UK) laboratories, Cheshire Innovation Park. Sample preparation 
was conducted by the author following a standardised procedure developed in-house at Shell Global Solutions (UK).

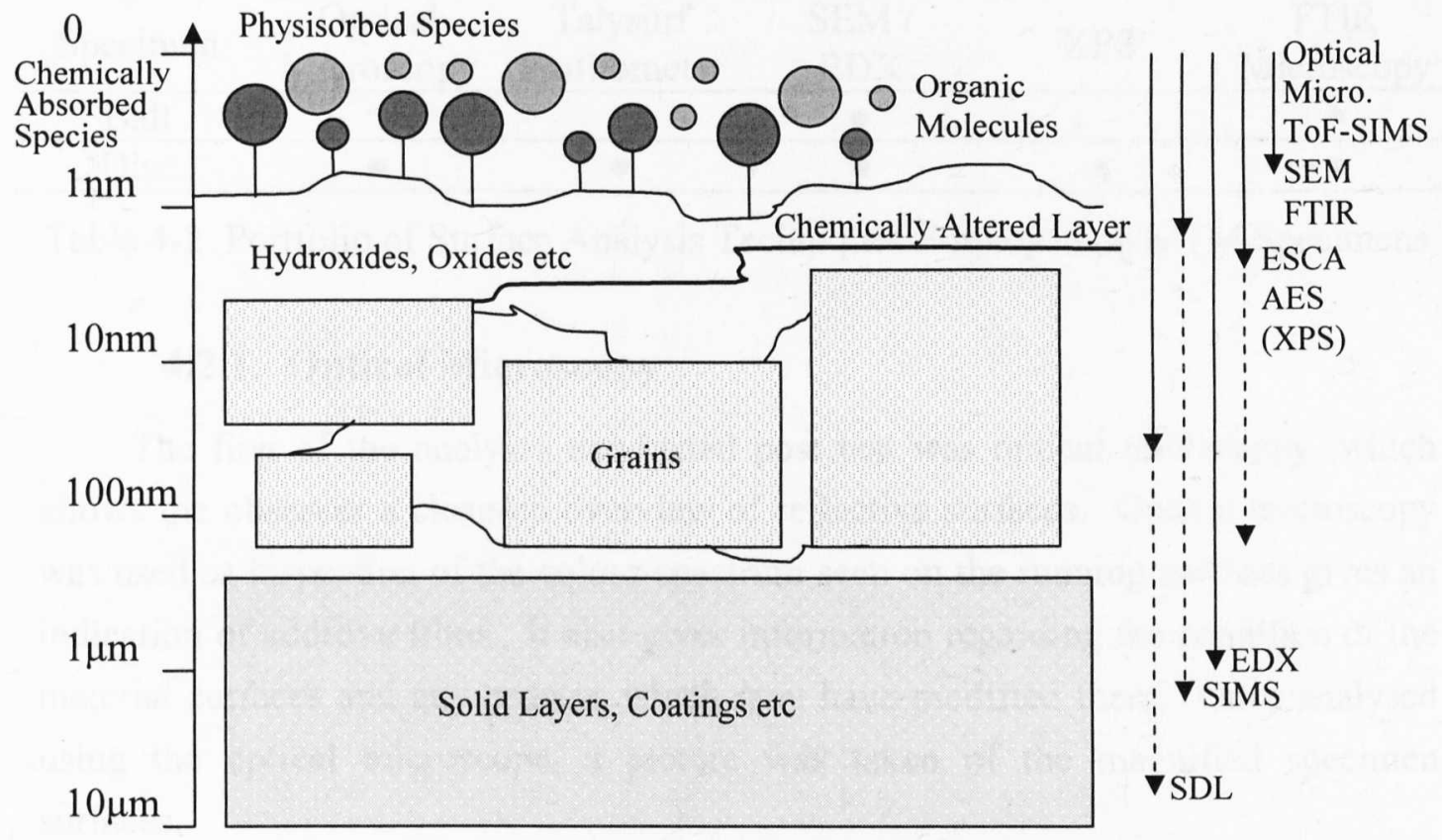

Figure 4-2 Specimen Surfaces and Analytical Techniques. After (Renondeau 2005).

Both prior to and post test sequence, specimens were placed in an ultrasonic Special Boiling Point (SBP) solvent bath for 30 minutes, the solvent being changed at 10 minute intervals. SBP solvent is a fluid made in-house at Shell Global Solutions (UK), specially designed for cleaning of apparatus such as the MTM machine and its specimens. The cleaning procedure is a standardised methodology recommended by Shell Global Solutions (UK) which serves to ensure homogeneity between specimens entering the MTM, and that surfaces are clean for post test analysis. Prior to X-ray Photon Spectroscopy (XPS) and Scanning Electron Microscopy / Energy Dispersive X-ray (SEM / EDX) the specimens were given a quick rinse for a few seconds under flowing heptane to ensure complete cleanliness was achieved. This was a particularly important procedure as it ensured the specimens were fit to enter highly sensitive equipment such as the XPS and SEM / EDX machines, where small amounts of contaminants not only affect the immediate analysis, but those that follow as well. Full details of the surface measurement employed by these machines is described in proceeding sections.

It was not practicable to analyse all specimens using all the selected surface analysis techniques. Due to the added complexity of surface analysis on the curved ball, surface analysis was only conducted using Fourier Transform Infra-red (FTIR) 
microscopy and SEM / EDX techniques on the balls. Table 4-2 details the surface analysis techniques employed on test specimens.

\begin{tabular}{cccccc}
\hline Specimen & $\begin{array}{c}\text { Optical } \\
\text { Microscopy }\end{array}$ & $\begin{array}{c}\text { Talysurf } \\
\text { Profilometer }\end{array}$ & $\begin{array}{c}\text { SEM/ } \\
\text { EDX }\end{array}$ & XPS & $\begin{array}{c}\text { FTIR } \\
\text { Microscopy }\end{array}$ \\
\hline Ball & & & $\bullet$ & & $\bullet$ \\
\hline Disc & $\bullet$ & $\bullet$ & $\bullet$ & $\bullet$ & $\bullet$ \\
\hline
\end{tabular}

Table 4-2 Portfolio of Surface Analysis Techniques Employed on MTM Specimens

\subsubsection{Optical Microscopy}

The first of the analyses conducted post test was optical microscopy, which allows the observer a close-up overview of reflective surfaces. Optical microscopy was used as inspection of the colour spectrum seen on the running surfaces gives an indication of additive films. It also gives information regarding the condition of the material surfaces and any process which may have modified them. Once analysed using the optical microscope, a picture was taken of the magnified specimen surfaces.

\subsubsection{Form Talysurf Contacting Profilometer Measurement}

After optical microscopy the disc specimens were analysed using a 'Series 2' Rank Taylor Hobson Form Talysurf contacting profilometer. The Form Talysurf instrument measures dimension, form and texture simultaneously by monitoring the vertical motion of the stylus tip using a laser referencing technique. Using powerful software, the vertical motion is mapped with data from the linear traverse creating immensely detailed maps of the analysed surface. In this investigation the machine was used to analyse the surface of the disc specimen only, in the manner described in Figure 4-3.

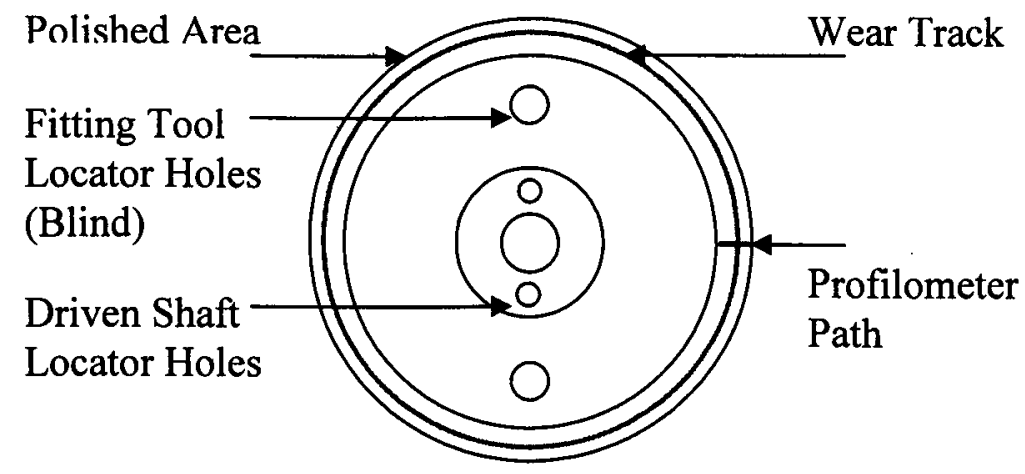

Figure 4-3 1:1 Scale Plan View of MTM Disc Specimen Detailing Profilometer 
The machine was used to give an indication of the wear profile and surface roughness of the disc, as well as information on the deposition of dense additive agglomerates on the surface. In early experiments a series of traces were taken around the wear track on the disc specimen. Analysis of these traces revealed that the wear track was very similar at all locations on the disc. For this reason, for latter tests a single profile trace was taken across the wear track of each disc specimen in as described in Figure 4-3.

\subsubsection{Scanning Electron Microscopy and Energy Dispersive X-ray Technique (SEM / EDX)}

Both the disc and ball specimens were analysed using the SEM/EDX technique. The SEM analysis method uses a focussed high energy electron beam which scans back and forth across the specimen surface. The highly energetic electrons strike the sample where various reactions can occur. The two principal forms of measurement take place using the so called 'secondary electrons' or 'backscattered electrons'. The term 'secondary electron' refers to the ionization of a specimen atom, caused by the passing of the high energy incident electron beam "near" to the atom. The beam passes near enough to a specimen atom to impart some of its energy to the specimen's lower energy electron, causing a deviation in the path of the incident electron beam. Each incident electron can produce several secondary electrons. The production of secondary electrons is very topographically related, as the energy carried by them is only sufficient to cause them to leave the surface of the specimen should they be very close to it $(\sim<10 \mathrm{~nm})$. The term 'backscattered electrons' refers to the occurrence of a direct collision between the incident electron and an atom within the specimen which is nearly normal to the incident path. This causes the incident electron to be scattered backwards 180 degrees. Before the beam moves to its next dwell-point an electron detector measures the number of interactions between the incident electron beam and the specimen. This information is then converted into a small electrical signal which is amplified and converted into an image of the scanned surface ( $O^{\prime}$ Connor, Sexton et al. 2003).

A spatial resolution of approximately 20 microns was used in the SEM and permitted scanning of the wear track in isolation, for comparison with a scan of the unworn specimen surface.

After SEM had been conducted on the specimens the Energy Dispersive X-ray technique was used. The basic premise behind the operation of EDX relies on the knocking off of electrons through interaction with the high energy electron beam. A position vacated by the removal of an inner shell electron is filled by a higher energy 
electron from the outer shell. For this to be possible the transferring outer electron must give up some of its energy by emitting an X-ray. The atom of every element releases X-rays with unique amounts of energy during the transferral process which establishes the identity of the atom from which it came. The EDX technique is not as surface orientated as the SEM measurement, as the X-rays are typically generated in a region in a region approximately 2 microns below the surface, although depth is obviously dependent on beam energy. The technique gives elemental analysis of the specimen substrate. For this investigation, the measurement was again carried out on two separate areas both inside and outside the specimen wear tracks (Vickerman 1997).

\subsubsection{X-ray Photon Spectroscopy (XPS)}

$\mathrm{X}$-ray Photon Spectroscopy is based on a phenomenon first outlined by Einstein in 1905, the photoelectric effect, where a photon beam is focused on the specimen undergoing analysis, resulting in the excitation of photoelectrons. The XPS technique is highly surface specific due to the short range of photoelectrons that are excited from the solid (Figure 4-2). The energy of the photoelectrons leaving the sample is measured to create a spectrum with a series of photoelectron peaks. The binding energy of the peaks are characteristic of each element. The composition of the specimen surface can be determined through analysis of the peak areas. The shape of each peak and the binding energy are slightly altered by the chemical state of the emitting atom, hence XPS can provide chemical bonding information. Thus, for the purpose of this investigation, the technique is ideal as it detects carbon and oxygen present in a typical oxidised metal surface and fatty acid surface film, but the chemical bonding information differentiates the two. The technique is sensitive to all elements except hydrogen and helium, suggesting the detection of thin fatty acid or amide films is possible (Vickerman 1997; O'Connor, Sexton et al. 2003).

XPS measurements are conducted in an Ultra High Vacuum (UHV) $\left(10^{-9} \mathrm{mbar}\right)$ condition to ensure atmospheric gas molecules are minimised and surface degradation is minute. Some geometric constraint is placed on components suitable for analysis. For the Machine used in this investigation the aperture for specimens is approximately $50 \mathrm{~mm}$ in diameter, making analysis of the $45 \mathrm{~mm}$ diameter MTM disc specimen difficult, but possible. For the measurements conducted on the MTM disc specimens the spot-size of the beam was $1 \mathrm{~mm}$ in diameter.

\subsubsection{Fourier Transform InfraRed Microscopy (FTIR)}

The operation of the FTIR microscopy has been previously discussed in Section 3.5.1. In this investigation, both the ball and disc specimens were highly reflective which aids the measurement technique by keeping the reflected portion of 
infrared energy as high as possible. The analyses employed on the specimens bears similarity with the SEM/EDX analysis, where measurements were made both on the wear track and away from it for comparative purposes. However, using this technique the beam 'spot' can be focussed down to a few microns, permitting the direct analysis of areas of interest such as the border between wear track and unworn surface.

\subsection{Mini Traction Machine Test}

Bearing in mind the three main aims of the MTM investigation; to gauge the performance of the friction modifier additives in the boundary regime, to investigate the performance of the friction modifier additives in the mixed regime and to create surface films for post test surface analysis, a bespoke program was developed. Guided by a series of initial tests and surface analyses the following operating conditions were selected.

- A lubricant reservoir temperature of $125^{\circ} \mathrm{C}$, to simulate the most arduous lubricant condition as guided by the ASTM D 6837 Sequence VI-B engine test, a test designed to measure the effects of automotive engine oils on the fuel economy of passenger cars.

- The ball was loaded onto the disc with a force of $2 \mathrm{~N}$, corresponding to a maximum Hertzian contact pressure of $0.5 \mathrm{GPa}$. This light load was selected for two reasons. Firstly, the grey cast iron disc is relatively soft in comparison with a standard AISI 52100 specimen, and wore heavily at higher load conditions in preliminary tests. Secondly, it was thought that a too heavy load might be detrimental to additive film formation, and might simply remove film from the surfaces leaving little for subsequent surface analysis.

- A Slide Roll Ratio (SRR) of $100 \%$ was selected for the tests. It is not ideal to run the machine at pure sliding conditions, as is the case at the piston assembly / cylinder liner interaction, as this may result in little surface film for surface analysis and high wear. Therefore a compromise was made and it was decided to run at what is considered to be an arduous condition in the MTM of $100 \%$ SRR. The Slide Roll Ratio is defined in this study by equation 6-1. For each measurement taken both the standard SRR (ball moving faster than disc) and the reciprocal SRR (disc moving faster than ball) were taken and averaged before a final traction coefficient was recorded. 

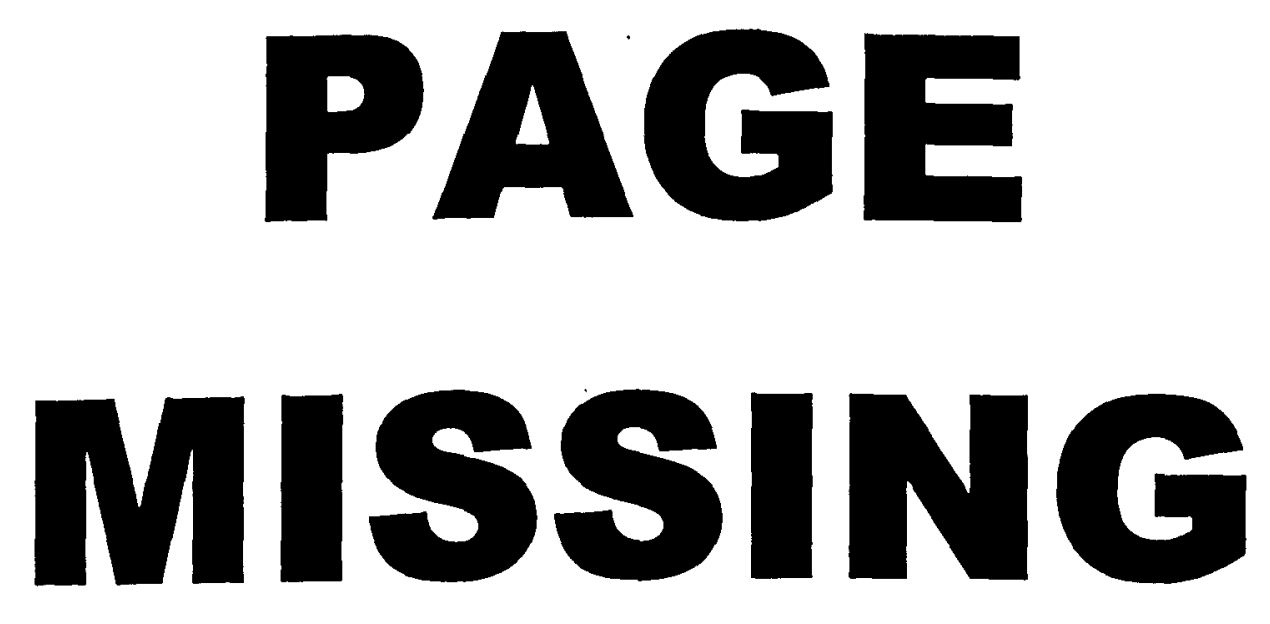

IN

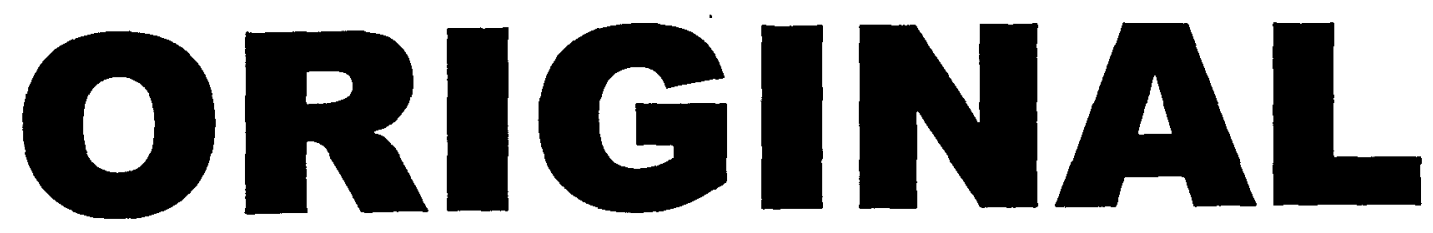


curve, optical microscope and profilometer data. However, only one set of specimens from each lubricant formulation test were analysed using SEM / EDX, XPS and FTIR microscope due to the costly nature of the techniques in both money and time.

\subsection{Test Lubricant Formulations}

Guided by the results of Chapter 3, it was decided that a limited number of blends should be tested on the MTM. For chemical comparison the best performing fatty acid and amide compounds were selected, as well as a blend of the two previously shown to have some beneficial synergistic effects. It was decided to blend the friction modifier additives in XHVI-8.2 ${ }^{\mathrm{TM}}$ (Shell) base oil as opposed to the XHVI-4TM (Shell) base oil used in the Chapter 3 tests, as it was felt the greater viscosity of XHVI-8.2 ${ }^{\mathrm{TM}}$ (Shell) would provide greater protection for the specimens throughout the bulk of the tests, and could help to limit wear. In addition, using a more viscous lubricant allows the sweeping of lubrication regimes rather than simply investigation into the boundary regime, as was the case in Chapter 3 . The selected friction modifier additives were blended using the method described in Chapter 3 , and in formulations detailed in Table 4-3. The dynamic viscosity of the lubricant blends was investigated by Russell Bannerman, a laboratory technician at Shell Global Solutions (UK), using a standard AR 1000 rheometer. The error associated with this type of measurement is thought to be $\pm 3 \%$, suggesting that the viscosity of the blended oils was not significantly affected by the presence of additive.

\begin{tabular}{cccc}
\hline Blend Name & Base Oil (wt\%) & FM Additive (wt\%) & $\begin{array}{c}\text { Dynamic Viscosity } \\
\text { at } 100^{\circ} \mathrm{C}(\mathrm{mPa} . \mathrm{s})\end{array}$ \\
\hline BASE & $100 \mathrm{wt} \%$ XHVI-8.2 & - & 6.9 \\
\hline LIN & $98 \mathrm{wt} \%$ XHVI-8.2 & $\begin{array}{c}\text { TM } \\
\text { tM Linoleic } \\
\text { Acid }\end{array}$ & 7.1 \\
\hline ERU & $98 \mathrm{wt} \%$ XHVI-8.2 & $2 \mathrm{wt} \%$ Erucylamide & 7.0 \\
\hline ERULIN & $98 \mathrm{wt} \%$ XHVI-8.2 & $\begin{array}{c}1 \mathrm{wt} \% \text { Linoleic } \\
\text { Acid }+\end{array}$ \\
& & $1 \mathrm{wt} \%$ Erucylamide \\
\hline
\end{tabular}

Table 4-3 Test Lubricant Formulations and Dynamic Viscosity at $100^{\circ} \mathrm{C}$ 


\subsection{MTM Friction Coefficient Measurement Results and}

\section{Analysis}

The friction coefficient data taken from the Mini Traction Machine was plotted against the entrainment velocity using a logarithmic scale creating what on initial inspection appears to be the left hand side of the 'Stribeck' curve.

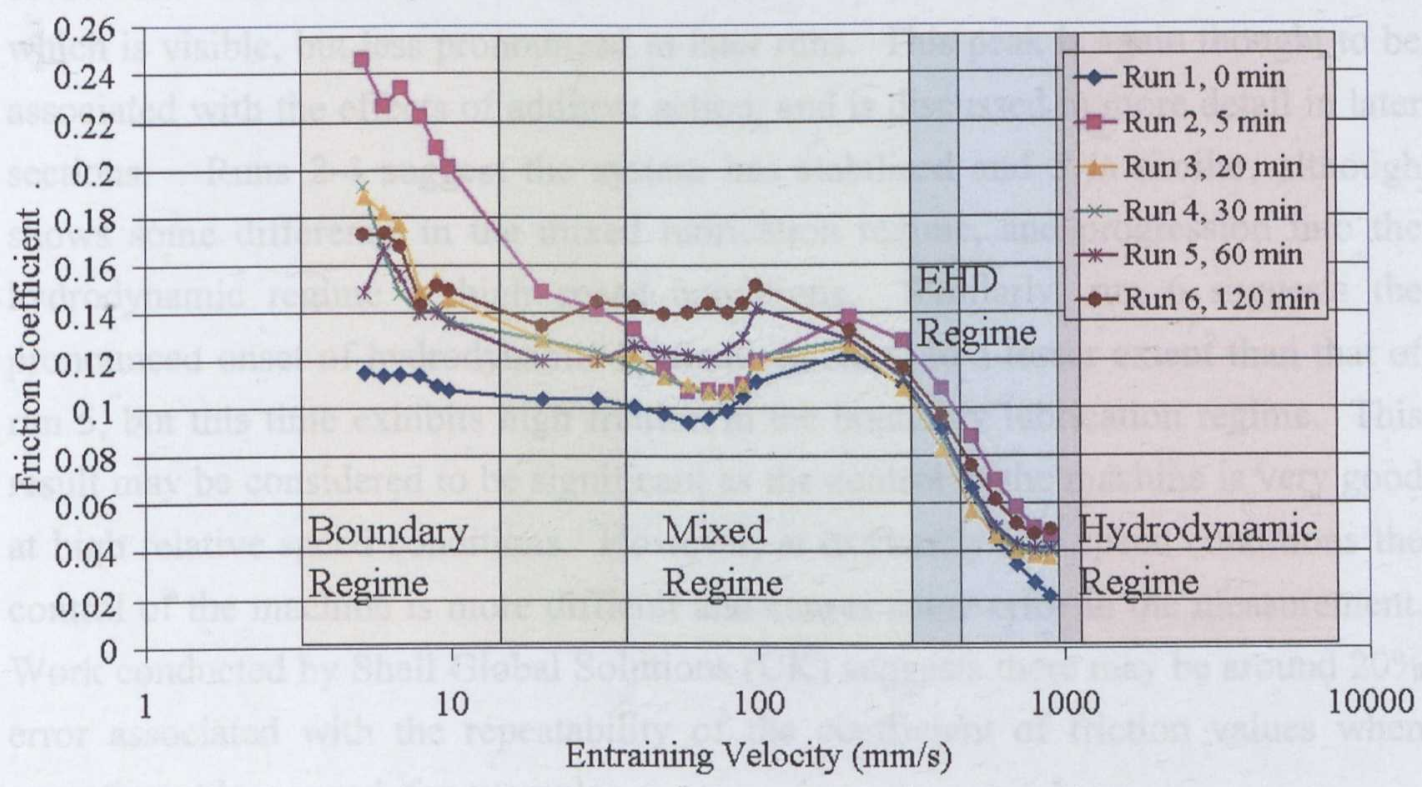

Figure 4-5 BASE: XHVI-8.2 ${ }^{\mathrm{TM}}$

It is impossible to accurately identify the points of transition between lubrication regimes, although Figure 4-5 details the progression of the contact from the hydrodynamic lubrication regime through to that of boundary lubrication. On closer inspection of the figure there is a subtle difference between run 1 taken at the start of the test sequence and the runs 2-6. The general trend of run 1 is of lower friction than the following runs. It is suggested that this is due to a mild running in process, where small amounts of material are thought to have been removed from the -surface of the relatively soft disc specimen creating a running track whose geometry is more locally conformal than that of the initial contact. In addition some running in of the ball specimen is also thought to have occurred, although it is considered to be insignificant when compared with that of the disc. Indeed it may also be considered that the sharp increase in coefficient of friction associated with run 2 , which occurs after a mean speed of $40 \mathrm{~mm} / \mathrm{s}$, could also be due to a running-in / wear effect, as it is believed that for this test the transition from the mixed regime to that of boundary occurs in this region. Runs 3-6 suggest the system has stabilised, and a more repeatable curve is produced. The final coefficient of friction associated with boundary contact is approximately 0.18 for pure XHVI-8.2 $2^{\mathrm{TM}}$ (Shell), run 6. 
Figure 4-6, for a blend containing Linoleic acid, suggests a different explanation to that of pure base oil. It is clear on initial inspection that the first run differs greatly from that of subsequent runs. In this case it is proposed that the reason for the dramatic change is not solely due to wear. It is suggested that the run 1 result concerns the formation of an additive film which is highly influential on the coefficient of friction. In addition at $\sim 9 \mathrm{~mm} / \mathrm{s}$ there is a sharp increase in friction which is visible, but less pronounced in later runs. This peak is again thought to be associated with the effects of additive action, and is discussed in more detail in later sections. Runs 2-4 suggest the system has stabilised and 5 is similar, although shows some difference in the mixed lubrication regime, and progression into the hydrodynamic regime at high speed conditions. Similarly, run 6 suggests the pronounced onset of hydrodynamic lubrication, albeit to a lesser extent than that of run 5, but this time exhibits high friction in the boundary lubrication regime. This result may be considered to be significant as the control of the machine is very good at high relative speed conditions. However, at extremely low speed conditions the control of the machine is more difficult and causes some error in the measurement. Work conducted by Shell Global Solutions (UK) suggests there may be around 20\% error associated with the repeatability of the coefficient of friction values when operating at low speed, for example.

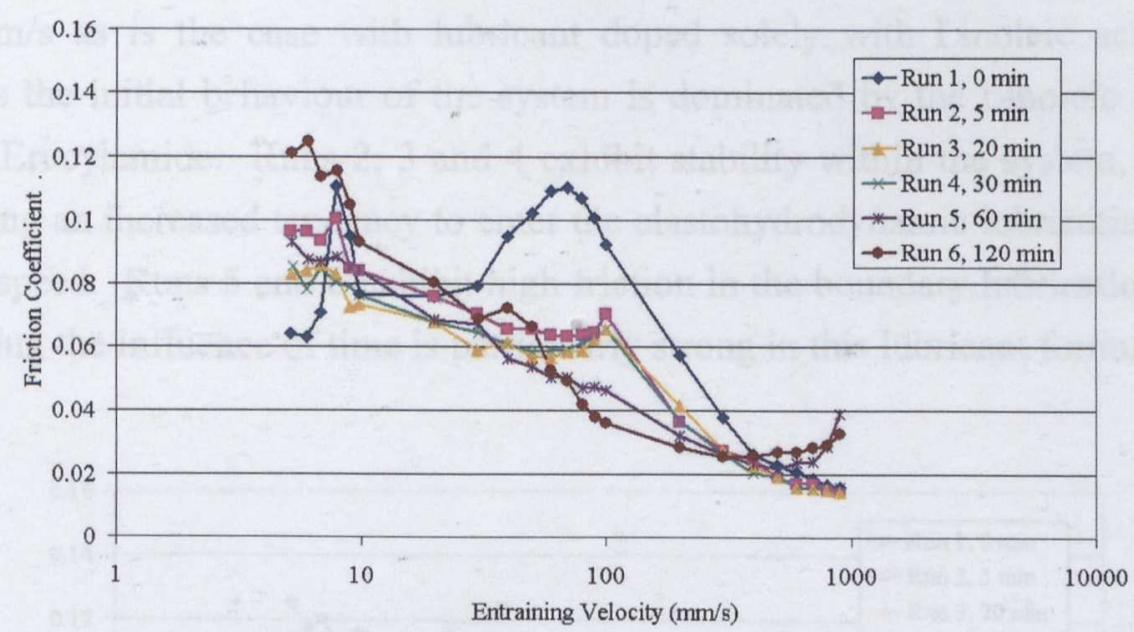

Figure 4-6 LIN: 2 wt\% Linoleic Acid in XHVI-8.2 ${ }^{\mathrm{TM}}$

The test of $2 \mathrm{wt} \%$ Erucylamide in base oil is shown in Figure 4-7. Again there is a visible difference between run 1 and runs 2-5, and run 6 appears to differ again. The difference between run 1 and subsequent runs is suggested to be due to the formation of an additive film as well as the standard running-in of the specimens. As shown in Figure 4-6, run 6 appears to be an outlier with respect to other runs and exhibits a pronounced elastohydrodynamic lubrication regime portion at high speed. At the boundary lubrication side of Figure 4-7 there appears to be an interesting 
event at $\sim 9 \mathrm{~mm} / \mathrm{s}$ where friction between the ball and disc is dramatically reduced. As in the figure depicting the test undertaken with Linoleic acid, Figure 4-6, run 6 exhibits extremely high friction in the boundary lubrication.

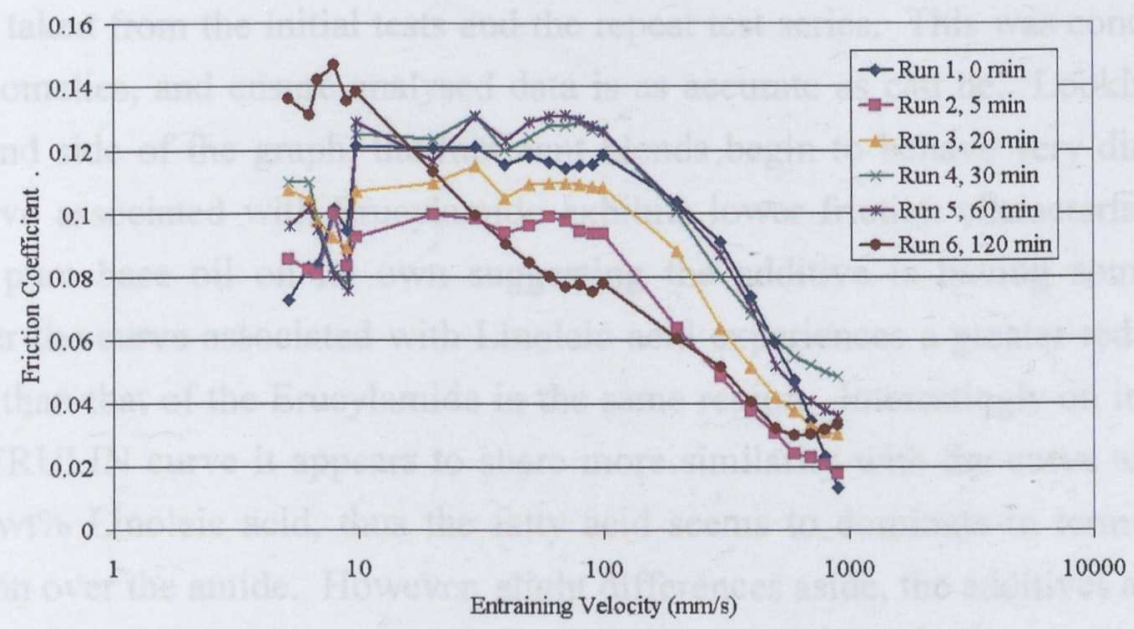

Figure 4-7 ERU: 2 wt\% Erucylamide in XHVI-8.2 ${ }^{\mathrm{TM}}$

The final graph in the test series, Figure 4-8, is for the base lubricant containing both $1 \mathrm{wt} \%$ Linoleic acid and $1 \mathrm{wt} \%$ Erucylamide. On consideration of run 1, the graph exhibits a trend not dissimilar to that of Figure 4-6 associated with 2 $\mathrm{wt} \%$ Linoleic acid in base oil. There is a clearly defined difference between this run and all proceeding. A high friction peak is seen at the $100 \mathrm{~mm} / \mathrm{s}$ location, and again at $9 \mathrm{~mm} / \mathrm{s}$ as is the case with lubricant doped solely with Linoleic acid. This suggests the initial behaviour of the system is dominated by the Linoleic acid, and not the Erucylamide. Runs 2, 3 and 4 exhibit stability within the system, although displaying an increased tendency to enter the elastohydrodynamic lubrication regime at high speed. Runs 5 and 6 exhibit high friction in the boundary lubrication regime suggesting the influence of time is particularly strong in this lubricant formulation.

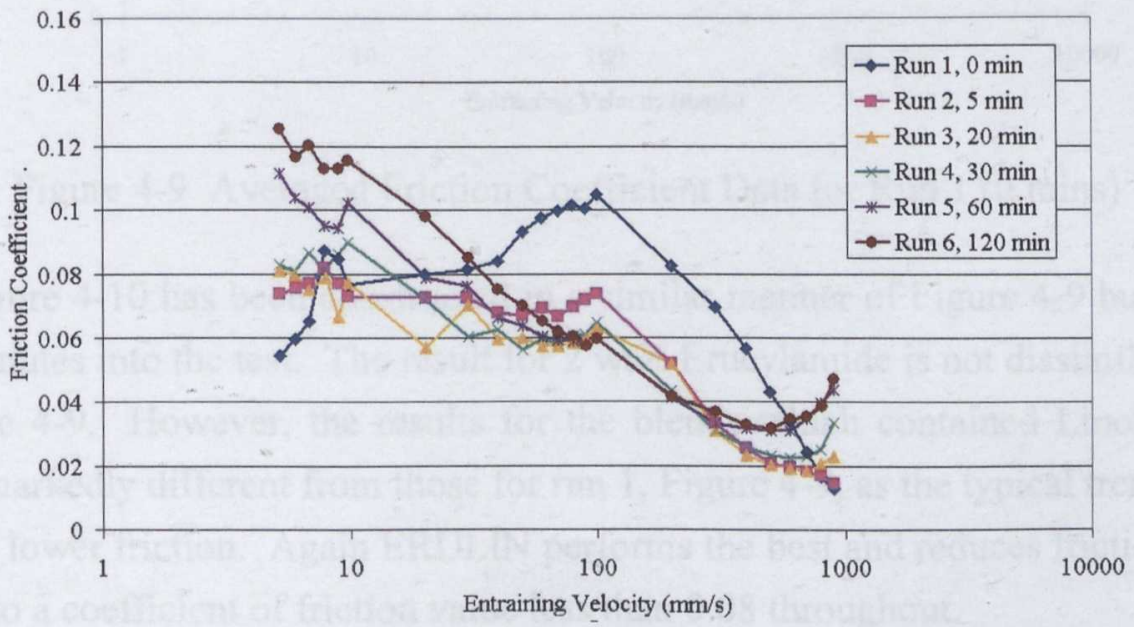

Figure 4-8 ERULIN: $1 \mathrm{wt} \%$ Erucylamide $+1 \mathrm{wt} \%$ Linoleic Acid in XHVI-8.2 ${ }^{\mathrm{TM}}$ 
Figure 4-9 depicts all the first runs of the test series together. It is thought that run 1 most accurately depicts the initial activation and forming of additive films. Each curve associated with friction modifier doped lubricant is the product of the average taken from the initial tests and the repeat test series. This was conducted to filter anomalies, and ensure analysed data is as accurate as can be. Looking at the right hand side of the graph, the lubricant blends begin to behave very differently. The curve associated with Erucylamide exhibits lower friction characteristics than that of pure base oil on its own suggesting the additive is having some effect. However the curve associated with Linoleic acid experiences a greater reduction in friction than that of the Erucylamide in the same region. Interestingly on inspection of the ERULIN curve it appears to share more similarity with the curve associated with 2 wt \% Linoleic acid, thus the fatty acid seems to dominate in terms of film formation over the amide. However, slight differences aside, the additives all reduce the coefficient of friction for the contact throughout the mixed and boundary lubrication regimes. The performance of ERULIN appears to reduce friction in the contact the most significantly of the three blends tested. In the boundary lubrication region ERULIN reduced friction in the contact by approximately $50 \%$ compared to base oil.

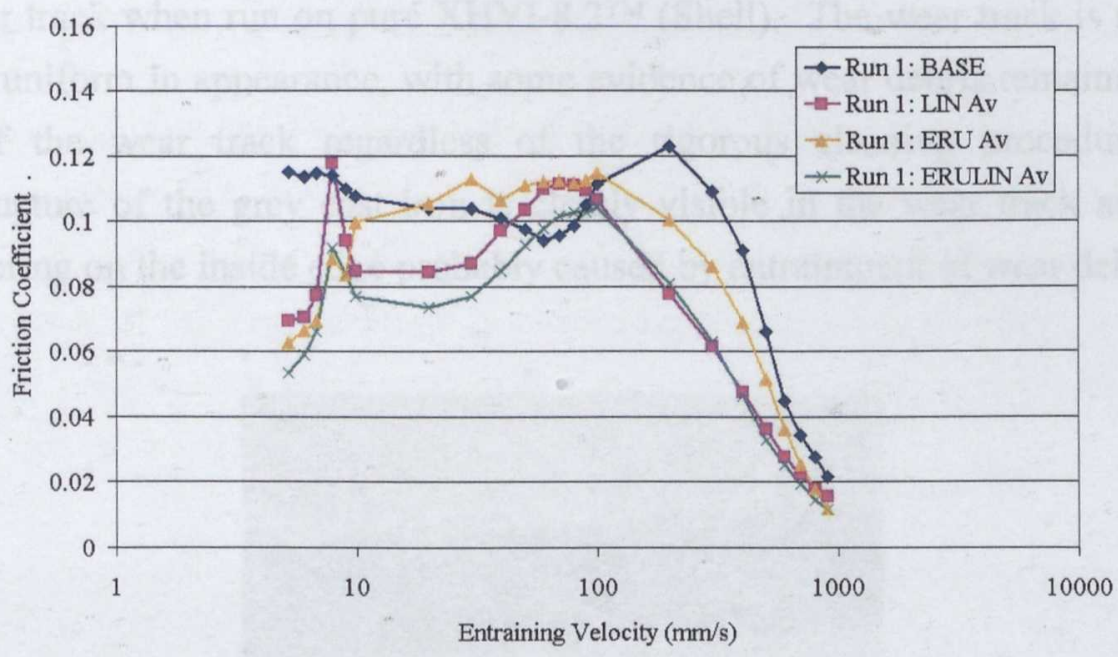

Figure 4-9 Averaged Friction Coefficient Data for Run 1 (0 mins)

Figure 4-10 has been constructed in a similar manner of Figure 4-9 but for run 4, 30 minutes into the test. The result for $2 \mathrm{wt} \%$ Erucylamide is not dissimilar to run 1, Figure 4-9. However, the results for the blends which contained Linoleic acid appear markedly different from those for run 1, Figure 4-9, as the typical trend is one of much lower friction. Again ERULIN performs the best and reduces friction in the contact to a coefficient of friction value less than 0.08 throughout. 


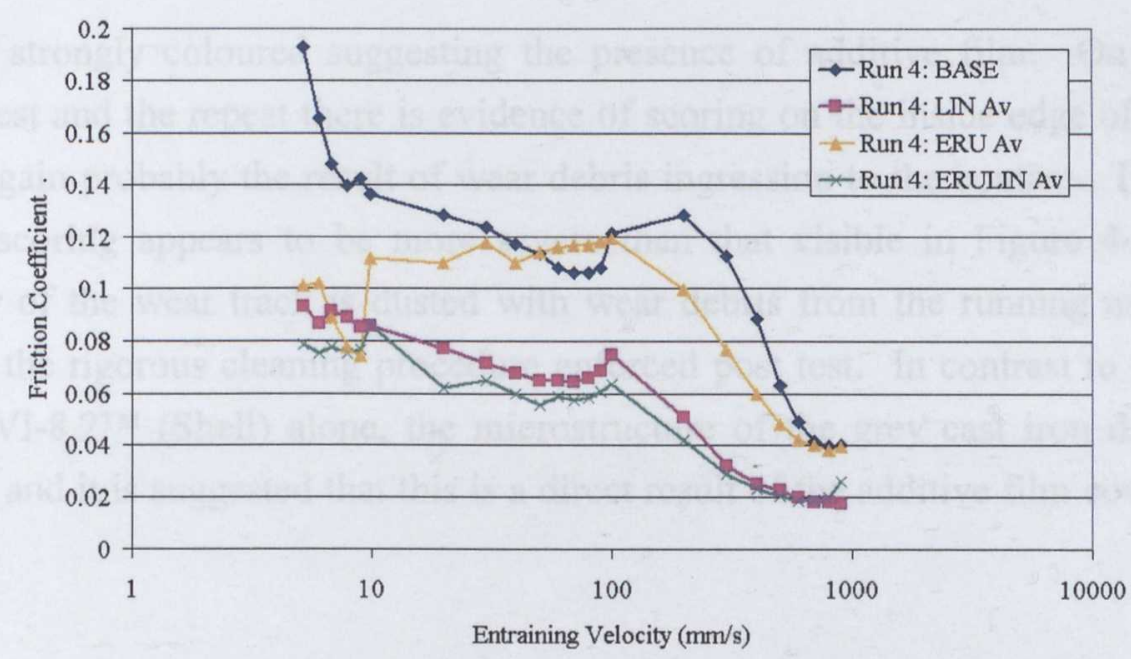

Figure 4-10 Averaged Friction Coefficient Data for Run 4 (30 mins)

\subsection{Optical Microscopy Results and Analysis}

To give an initial indication of state of the disc specimens post test, optical microscopy was performed. On initial inspection of Figure 4-11 - Figure 4-14 it is clear there is a marked difference between the surface of discs run with friction modifier additive and that of the disc run solely on base oil. Figure 4-11 depicts the disc wear track when run on pure XHVI-8.2 ${ }^{\mathrm{TM}}$ (Shell). The wear track is relatively thin and uniform in appearance, with some evidence of wear debris remaining at the edges of the wear track regardless of the rigorous cleaning procedure. The microstructure of the grey cast iron is clearly visible in the wear track area, as is some scoring on the inside edge probably caused by entrainment of wear debris.

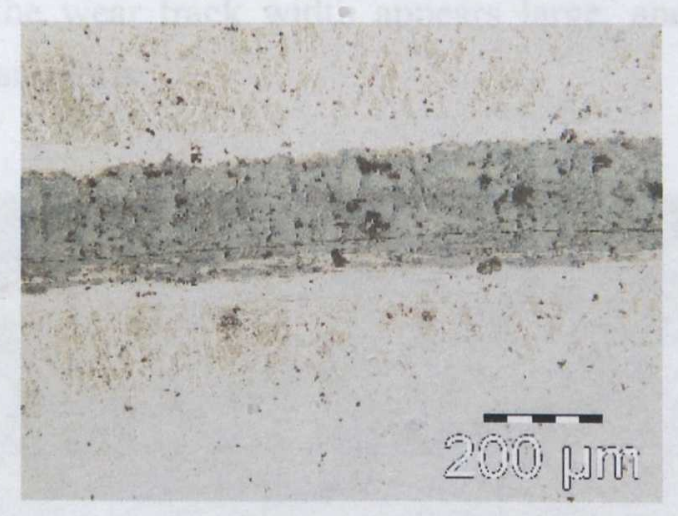

Figure 4-11 BASE Post Test Disc Surface

Figure 4-12 is a micrograph of disc surfaces when run on lubricant containing $2 \mathrm{wt} \%$ Linoleic acid. The difference between this picture and that of the surface run on pure base oil is startling. The width of the wear track appears greater, and the 
surface strongly coloured suggesting the presence of additive film. On both the initial test and the repeat there is evidence of scoring on the inside edge of the wear track, again probably the result of wear debris ingression to the contact. The extent of the scoring appears to be more severe than that visible in Figure 4-11. The exterior of the wear track is dusted with wear debris from the running area, again despite the rigorous cleaning procedure enforced post test. In contrast to when run on XHVI-8.2 $2^{\mathrm{TM}}$ (Shell) alone, the microstructure of the grey cast iron disc is not visible, and it is suggested that this is a direct result of the additive film covering the surface.

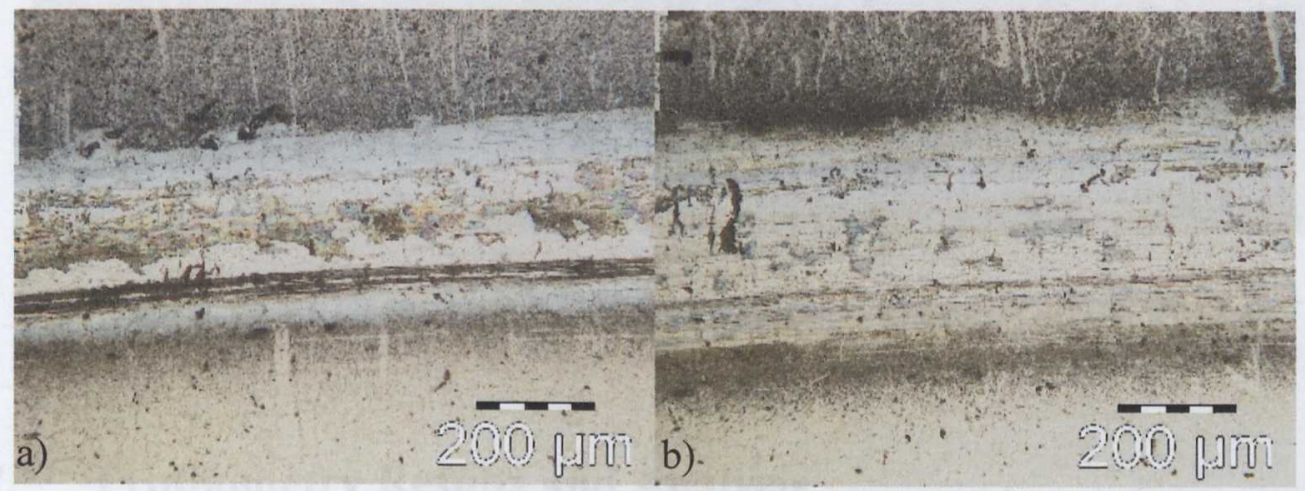

Figure 4-12 LIN Post Test Disc Surfaces - a) First Test, b) Repeat

The surfaces of the discs run with Erucylamide (Figure 4-13) again are strongly coloured suggesting the presence of additive, although the film appears to be mainly concentrated at the edges of the wear track. In the centre of the wear track the microstructure of the disc substrate is visible suggesting minimal film formation, if indeed any. Again the wear track width appears large, and with clearly defined edges dusted with wear debris.

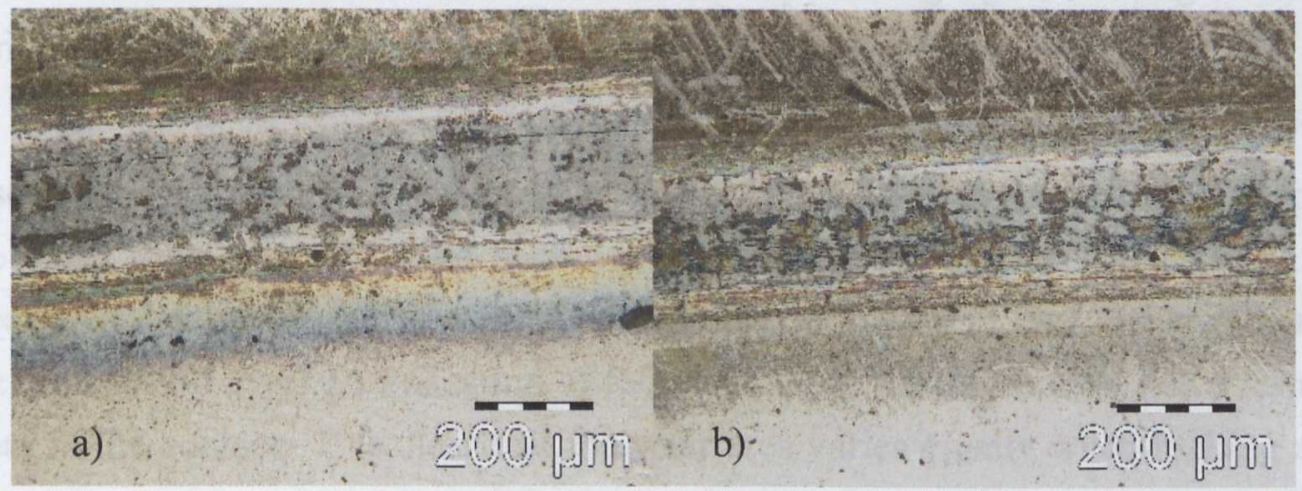

Figure 4-13 ERU Post Test Disc Surfaces - a) First Test, b) Repeat 
Figure 4-14 has many features in common with the two preceding figures, which also feature a colourful exterior. Colour thought to indicate additive presence is mainly visible in the centre of the wear track, suggesting more likeness to discs run with Linoleic acid alone. However, the edges of the wear track are not always clear, and some large deposits appear to line them.

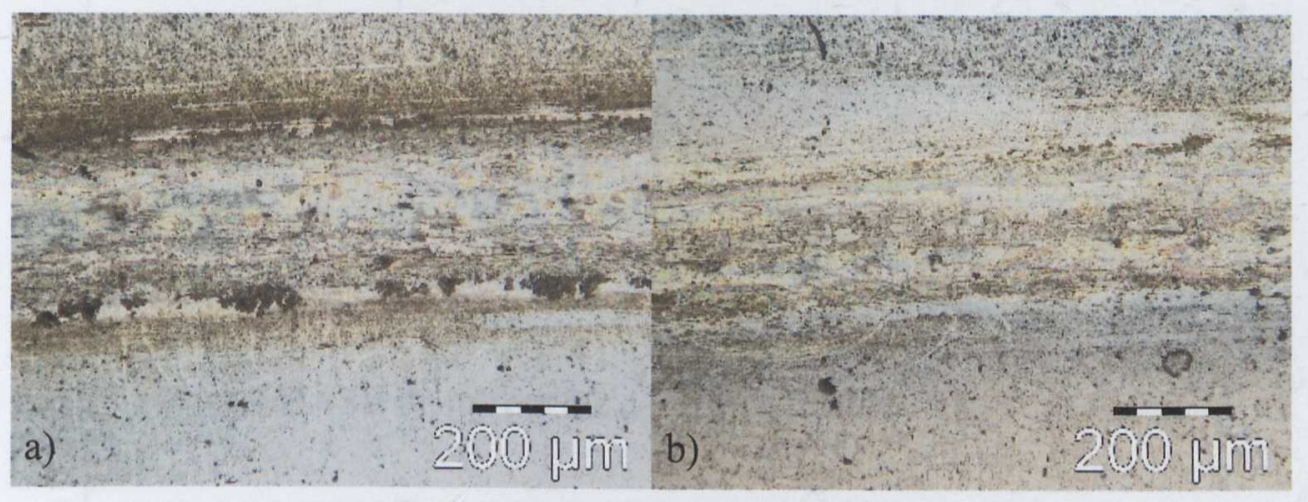

Figure 4-14 ERULIN Post Test Disc Surfaces - a) First Test, b) Repeat

\subsection{Profilometry Results and Analysis}

A single trace was taken on each disc specimen across the wear track area using a Taylor Hobson Series 2 contacting Profilometer. System software then calculated the shaded area of the 'wear hole' for each trace, displayed in the corner of Figure 4-15 - Figure 4-18, and also summarised in Table 4-4 along with repeat run data.

The wear profiles appear typical for the sliding / rolling contact between sphere and flat plate. It is clear from Figure 4-16, which features the wear track created with lubricant containing Linoleic Acid, that there are peaks of deposit outlining the wear track area. These are also visible in the superseding figure relating to the test conducted with Erucylamide doped lubricant.

The key piece of information to be taken from the profilometry is the wear area associated with each test (Table 4-4). The right-hand column of the table displays the mean wear area calculated from consideration of the first and repeat test. The wear area data is a result of a calculation based on the direct trace measurement of the profile, and gives an indication of the amount of material removed as a result of wear in the contact. It is clear that the wear area varies greatly when looking at all tests in comparison. The least wear occurs in the test conducted with Linoleic acid as a friction modifier, which reduces disc specimen wear by approximately $38 \%$ compared with the result for base oil alone. The test conducted with both Linoleic 
acid and Erucylamide exhibits slightly more wear than Linoleic acid on its own. The base lubricant on its own appears to give the third highest amount of wear, only exceeded by the test which featured Erucylamide as friction modifier which exhibited a wear area nearly twice as great.

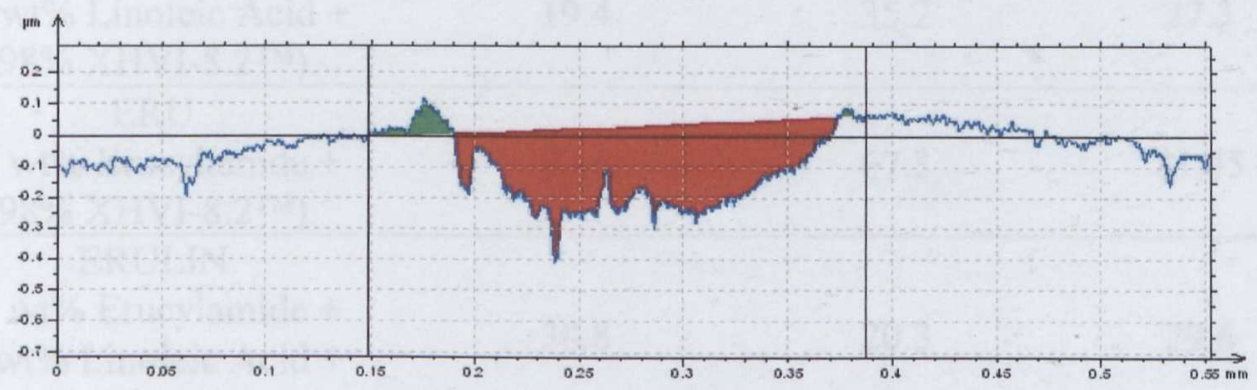

Figure 4-15 BASE Post Test Disc Surface Profilometry Measurement

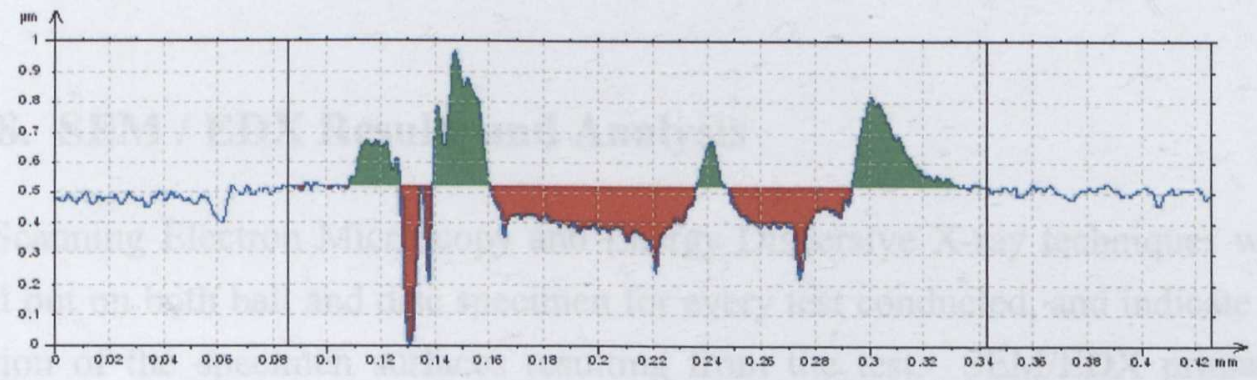

Figure 4-16 LIN Post Test Disc Surface Profilometry Measurement

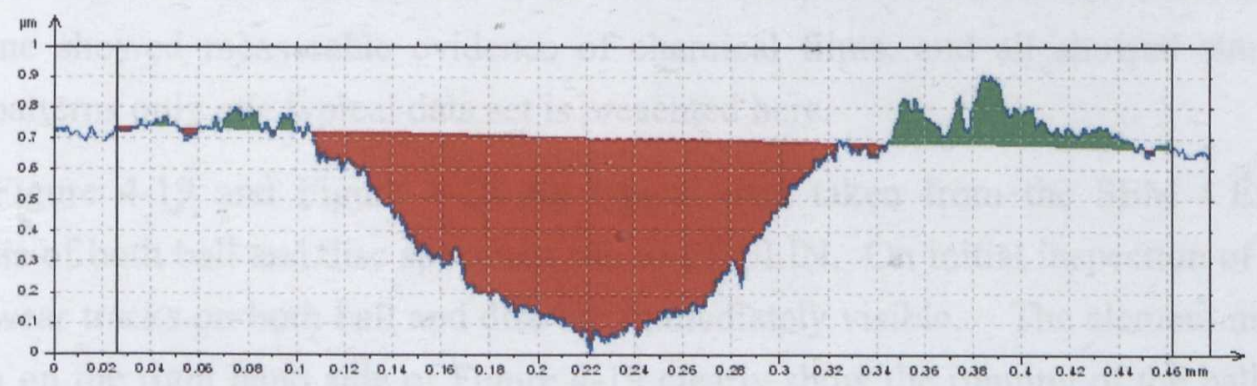

Figure 4-17 ERU Post Test Disc Surface Profilometry Measurement

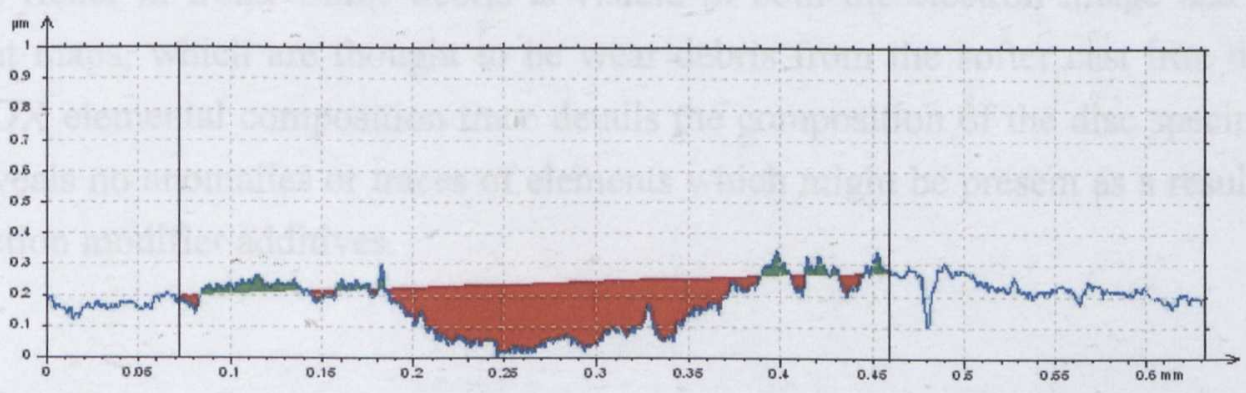

Figure 4-18 ERULIN Post Test Disc Surface Profilometry Measurement 
It is clear on initial inspection of the above figures that the contact between the ball and disc specimen has caused the disc to wear.

\begin{tabular}{|c|c|c|c|}
\hline Blend & $\begin{array}{c}\text { First Test Wear } \\
\text { Area }\left(\mu \mathrm{m}^{2}\right)\end{array}$ & $\begin{array}{c}\text { Repeat Test Wear } \\
\text { Area }\left(\mu \mathrm{m}^{2}\right)\end{array}$ & $\begin{array}{l}\text { Mean Wear } \\
\text { Area }\left(\mu \mathrm{m}^{2}\right)\end{array}$ \\
\hline BASE & 43.9 & - & $(43.9)$ \\
\hline $\begin{array}{c}\text { LIN } \\
\text { (2 wt } \% \text { Linoleic Acid + } \\
\left.98 \% \text { XHVI-8.2 }{ }^{\mathrm{TM}}\right) \\
\end{array}$ & 19.4 & 35.2 & 27.3 \\
\hline $\begin{array}{c}\text { ERU } \\
(2 \mathrm{wt} \% \text { Erucylamide }+ \\
98 \% \text { XHVI-8.2 } \\
\end{array}$ & 95.6 & 67.3 & 81.45 \\
\hline $\begin{array}{c}\text { ERULIN } \\
\text { (1 wt\% Erucylamide }+ \\
1 \text { wt } \% \text { Linoleic Acid }+ \\
98 \% \text { XHVI-8.2 }\end{array}$ & 38.8 & 20.3 & 29.6 \\
\hline
\end{tabular}

Table 4-4 Wear Area Measurements

\subsection{SEM / EDX Results and Analysis}

Scanning Electron Microscopy and Energy Dispersive X-ray techniques were carried out on both ball and disc specimen for every test conducted, and indicate the condition of the specimen surfaces resulting from the test. SEM/EDX primarily investigated the wear occurring on the specimen surfaces and also looked for evidence of chemical films. As none of the samples tested in the SEM/EDX machine showed measurable evidence of chemical films, and all showed similar wear patterns only one typical data set is presented here.

Figure 4-19 and Figure 4-20 are typical data taken from the SEM / EDX analysis of both ball and disc specimen run in ERULIN. On initial inspection of the data, wear tracks on both ball and disc are immediately visible. The element maps shown on the right hand side of Figure 4-19 clearly show the running of the ball on the plate causes the carbon from the surface of the ball to be removed, exposing a surface richer in iron. Some debris is visible in both the electron image and the element maps, which are thought to be wear debris from the softer cast iron disc. The EDX elemental composition trace details the composition of the disc specimen and reveals no anomalies or traces of elements which might be present as a result of the friction modifier additives. 

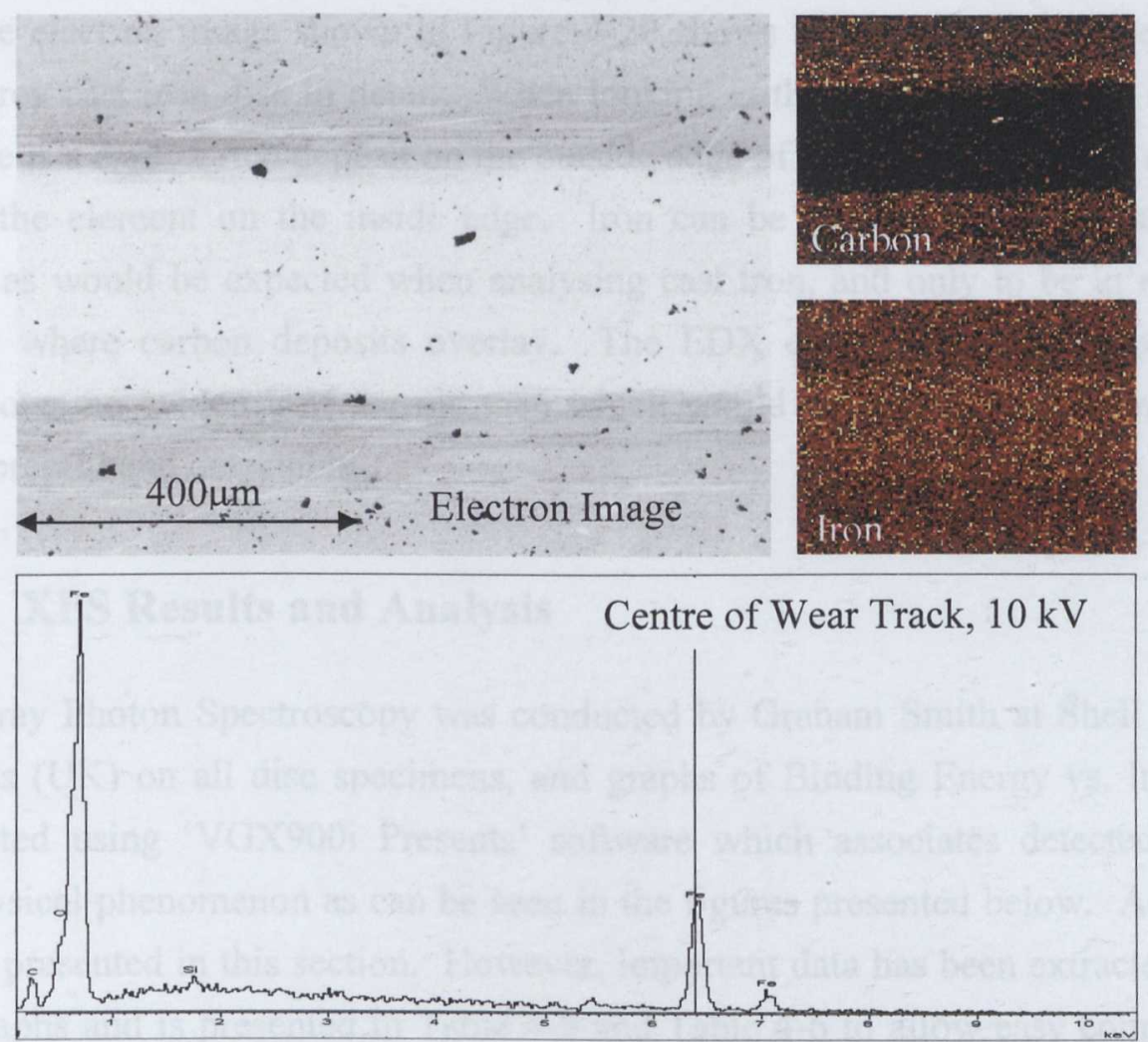

Figure 4-19 ERULIN Post Test Ball Surface SEM / EDX Analysis
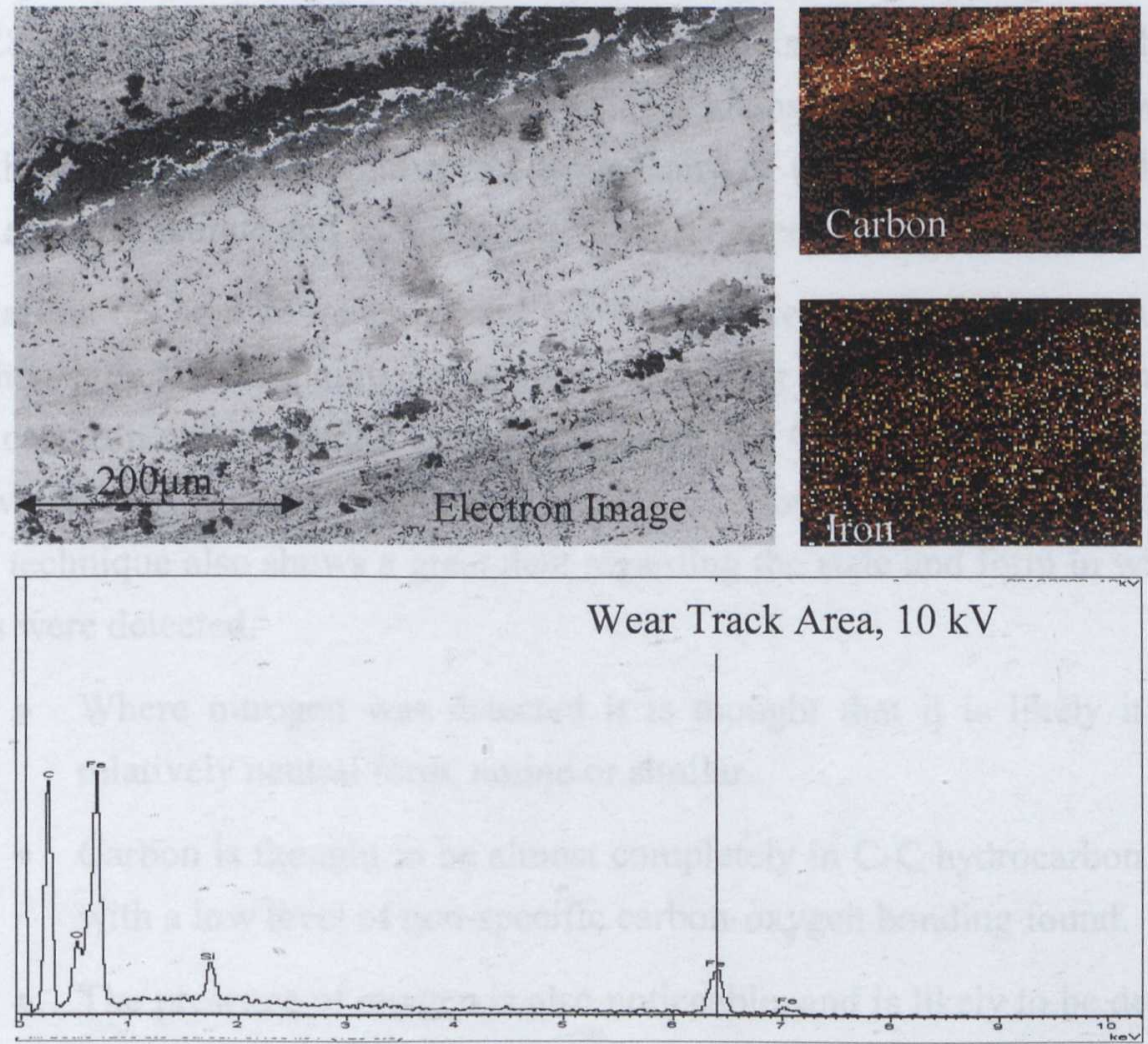

Figure 4-20 ERULIN Post Test Disc Surface SEM / EDX Analysis 
The electron image shown in Figure 4-20 shows the corresponding wear scar on the grey cast iron disc in detail. When looking at the elemental maps it is clear that there is a carbon rich deposit on the outside edge of the wear track and a relative lack of the element on the inside edge. Iron can be seen to populate the entire surface, as would be expected when analysing cast iron, and only to be in reduced amounts where carbon deposits overlay. The EDX elemental composition trace again shows no evidence of the nitrogen which would be present should an amide film be present and detectable.

\subsection{XPS Results and Analysis}

X-ray Photon Spectroscopy was conducted by Graham Smith at Shell Global Solutions (UK) on all disc specimens, and graphs of Binding Energy vs. Intensity constructed using 'VGX900i Presents' software which associates detected peaks with physical phenomenon as can be seen in the figures presented below. A typical result is presented in this section. However, important data has been extracted from these graphs and is presented in Table 4-5 and Table 4-6 to allow easy comparison of the entire data set.

Figure 4-21 and Figure 4-22 are typical examples of the processed data taken from the XPS analysis. The data has been 3-point smoothed and charge-corrected to $\mathrm{C} 1 \mathrm{~s}=285.0 \mathrm{eV}$. The binding energies of the peaks are estimated by the XPS software and represent the point of highest signal intensity within the peak window. Due to the relatively low signal levels from many of the species present the peak energies are not accurate and should not be used for chemical state estimates.

Analysis of the data taken on and off the disc wear track for all the lubricant blends shows the XPS technique is capable of detecting the elements which make up the grey cast iron disc specimen. Iron, Manganese and Carbon were all detected and are known to be present in the substrate material. However, the information from the XPS technique also shows a great deal regarding the state and form in which the elements were detected.

- Where nitrogen was detected it is thought that it is likely it is in a relatively neutral form, amine or similar.

- Carbon is thought to be almost completely in C-C hydrocarbon groups, with a low level of non-specific carbon-oxygen bonding found.

- The presence of oxygen is also noticeable, and is likely to be detectable in the oxide form, as a surface film. 
- Sulphur was also detected at low levels, and is likely to be in an oxidised form, as sulphate or similar.

- The X-ray excited Na KLL Auger peak was also detected, but the Na 1s peak was too weak for quantification.

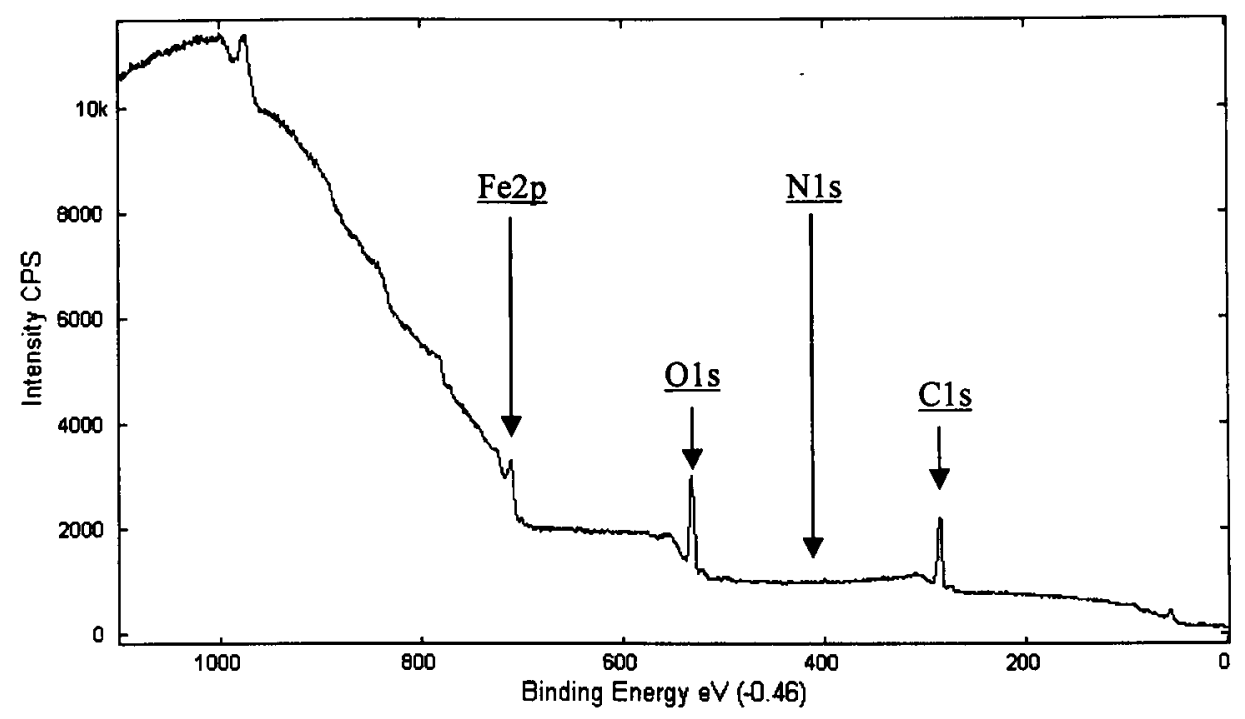

Figure 4-21 BASE Post Test Disc Surface XPS Analysis On Wear Track

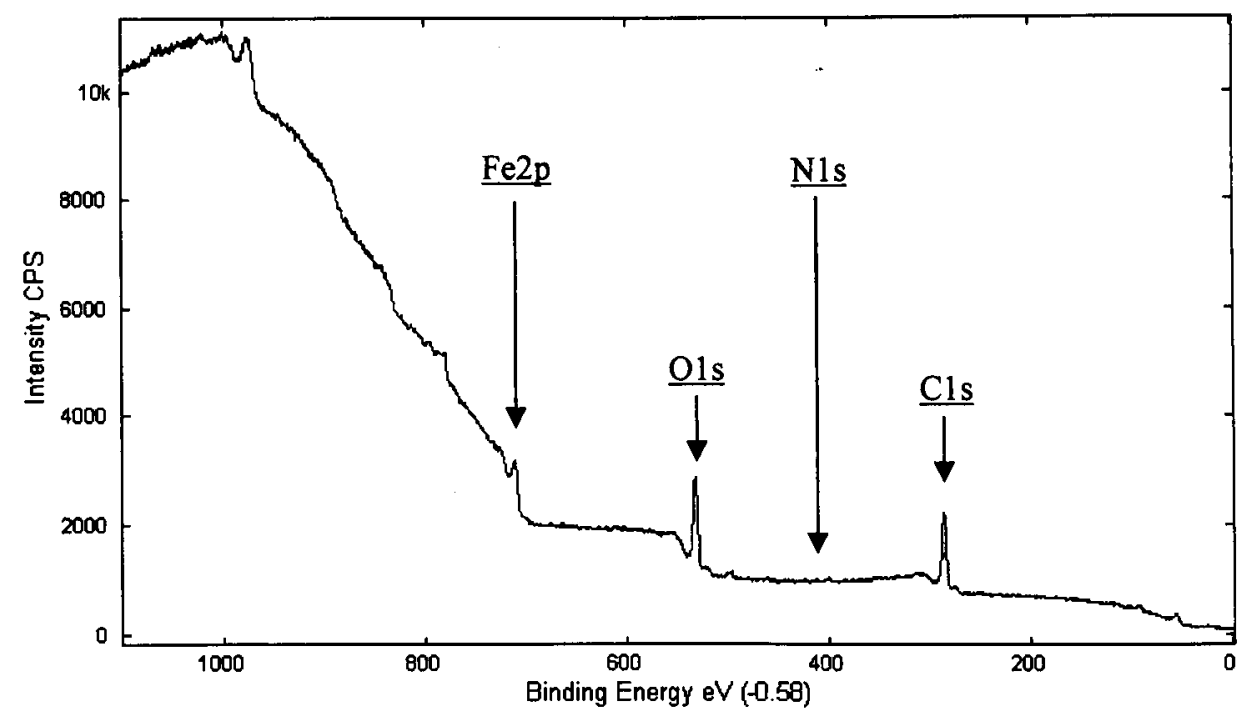

Figure 4-22 BASE Post Test Disc Surface XPS Analysis Off Wear Track

It is clear from Table 4-5 that there are some differences associated with the concentration of each element detected on the disc specimens. There are subtle differences between the quantities of each element found on and off the wear track. 
Looking at the data associated with the test conducted using only pure base oil, it is clear that proportionally more iron and carbon species are detectable off the wear track than on it, and that more oxygen species are detectable on the wear track than off.

Table 4-6 shows there are some differences in the binding energies of species detected on and off the wear track. For example, looking at the data associated with the Linoleic acid test there is a difference in the binding energy of the O1s peak in this test data set when compared with any other of 1-2 electron volts, which is thought to be significant.

Where Erucylamide was the friction modifier employed there is little difference between the on and off wear track binding energies, although some significance may be placed on the result suggesting an increase of N1s binding energy off the wear track. However, it is interesting to note that the typical N1s binding energy detected on the disc run with amide is significantly lower than was detected on any other specimen, suggesting a slight difference in the Nitrogen state.

\begin{tabular}{|c|c|c|c|c|c|c|c|c|}
\hline & \multicolumn{2}{|c|}{$\begin{array}{l}\text { BASE } \\
\text { XHVI-8.2 } \\
\text { Conc. }(\%)\end{array}$} & \multicolumn{2}{|c|}{$\begin{array}{c}\text { LIN } \\
\text { XHVI-8.2 } \\
2 \mathrm{wt} \% \text { Linoleic } \\
\text { Acid } \\
\text { Conc. (\%) }\end{array}$} & \multicolumn{2}{|c|}{$\begin{array}{c}\text { ERU } \\
\text { XHVI-8.2 } 2^{\mathrm{TM}}+ \\
2 \text { wt\% Erucylamide } \\
\text { Conc. }(\%)\end{array}$} & \multicolumn{2}{|c|}{$\begin{array}{c}\text { ERULIN } \\
\text { XHVI-8.2 } \\
1 \mathrm{wt} \% \\
\text { Erucylamide + } \\
1 \text { wt } \% \text { Linoleic } \\
\text { Acid } \\
\text { Conc. }(\%) \\
\end{array}$} \\
\hline Track & On & Off & On & Off & On & Off & On & Off \\
\hline $\mathrm{Nals}$ & & & & & 0.9 & 1.9 & 0.6 & 0.6 \\
\hline $\mathrm{Fe} 2 \mathrm{p}$ & 5.0 & 5.5 & 1.2 & 0.5 & 3.5 & 3.5 & 0.5 & \\
\hline $\mathrm{Mn} 2 \mathrm{p} 3 / 2$ & 0.29 & & & & 0.1 & & & \\
\hline O1s & 28.9 & 27.4 & 23.4 & 21.2 & 18.8 & 20.9 & 15.0 & 14.1 \\
\hline N1s & 1.4 & 1.7 & & 0.7 & 3.4 & 3.3 & 4.1 & 4.1 \\
\hline C1s & 64.4 & 65.4 & 75.2 & 77.7 & 73.3 & 69.8 & 79.7 & 81.2 \\
\hline $\mathrm{S} 2 \mathrm{p}$ & & & 0.2 & & & 0.7 & & \\
\hline $\mathrm{Na}$ LKK & & * & & & & & & \\
\hline
\end{tabular}

Table 4-5 Average Atomic \% Over Information Depth

When analysing the result associated with the ERULIN test, it is interesting to note the bulk change in the O1s binding energy with respect to other analyses, which took place without acidic friction modifier. Interestingly there is no shift in the binding energy of the nitrogen species even though amide was present. 


\begin{tabular}{|c|c|c|c|c|c|c|c|c|}
\hline & \multicolumn{2}{|c|}{$\begin{array}{c}\text { BASE } \\
\text { XHVI-8.2 } \\
\text { Binding Energy } \\
(\mathrm{eV})\end{array}$} & \multicolumn{2}{|c|}{$\begin{array}{c}\text { LIN } \\
\text { XHVI-8.2 } \\
2 \text { wt\% }+ \\
\text { Linoleic } \\
\text { Acid } \\
\text { Binding Energy } \\
(\mathrm{eV})\end{array}$} & \multicolumn{2}{|c|}{$\begin{array}{c}\text { ERU } \\
\text { XHVI-8.2 } 2^{\mathrm{TM}}+ \\
2 \mathrm{wt} \% \text { Erucylamide } \\
\text { Binding Energy } \\
(\mathrm{eV})\end{array}$} & \multicolumn{2}{|c|}{$\begin{array}{c}\text { ERULIN } \\
\text { XHVI-8.2 } \\
1 \mathrm{wt} \% \\
\text { Erucylamide }+ \\
1 \mathrm{wt} \% \text { Linoleic } \\
\text { Acid } \\
\text { Binding Energy } \\
(\mathrm{eV}) \\
\end{array}$} \\
\hline $\begin{array}{l}\text { Wear } \\
\text { Track }\end{array}$ & On & Off & On & Off & On & Off & On & Off \\
\hline $\mathrm{Nals}$ & & & & & 1071.1 & 1071.0 & 1071.1 & 1070.5 \\
\hline $\mathrm{Fe} 2 \mathrm{p}$ & 711.0 & 710.9 & 712.2 & 709.5 & 711.1 & 711.0 & 712.1 & \\
\hline $\mathrm{Mn} 2 \mathrm{p} 3 / 2$ & 642.0 & & & & 641.1 & & & \\
\hline O1s & 531.5 & 531.4 & 532.7 & 534.0 & 531.6 & 531.5 & 532.1 & 532.0 \\
\hline N1s & 400.5 & 400.4 & & 401.0 & 399.1 & 399.5 & 400.1 & 400.5 \\
\hline Cls & 285.0 & 284.9 & 285.2 & 286.5 & 285.1 & 285.0 & 285.1 & 285.0 \\
\hline $\mathrm{S} 2 \mathrm{p}$ & & & 167.2 & & & 167.0 & & \\
\hline $\mathrm{Na}$ LKK & & * & & & & & & \\
\hline
\end{tabular}

Table 4-6 Binding Energy Spectra

Of greater interest is the comparison of data between tests. It is clear that more iron is visible on the disc surface where no additive is used than in tests where additive doped lubricants were employed. This fact is also true for the presence of oxygen, suggesting more oxide layer is visible where no additives have been used. An increase in the detectable levels of Nitrogen was seen where amide doped lubricant was used, and an almost complete lack of Nitrogen detected where Linoleic acid was used. An increase in the measured concentration of carbon between the base tests and those which took place with additive doped lubricant was seen throughout the test series. It is worth mentioning at this point that any following discussion relating to the comparison of one data set to the other with respect to concentration of species on the surface should be done so with care. Figures relating to concentrations are quoted in percentage values of the total surface, and are not in absolute terms. For this reason, only large changes in species concentration values can be taken considered with any certainty.

\subsection{FTIR Microscopy Results and Analysis}

FTIR Microscopy was conducted by Rob Wetton at Shell Global Solutions (UK) on all specimens, graphs of the spectral absorption of the surfaces are seen in Figure 4-23 to Figure 4-26.

The absorbance spectra of base on the ball sample oil is shown in Figure 4-23, microscopy of the disc specimen did not show any significant spectra and so has been omitted from this report. The main peaks of absorbance are marked on the 
diagram, the most noticeable sets of peaks around the $2900 \mathrm{~cm}^{-1}$ region and those seen around the $1500 \mathrm{~cm}^{-1}$ are related to the hydrocarbon structure of the base oil. As such, this trace can be considered to be a benchline for interpretation of preceding figures.

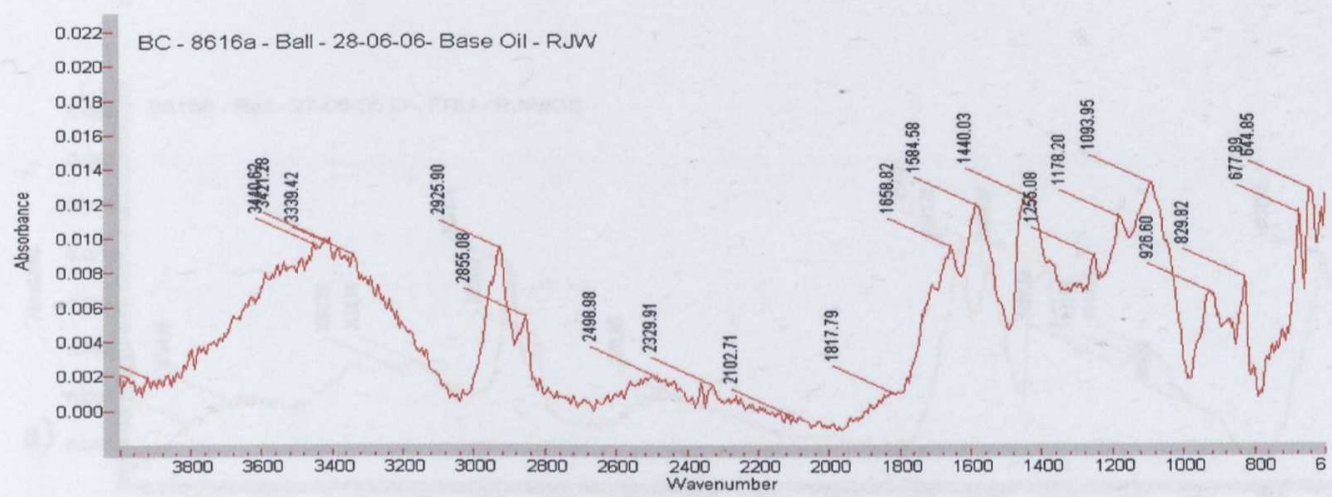

Figure 4-23 BASE Post Test Ball FTIR Analysis
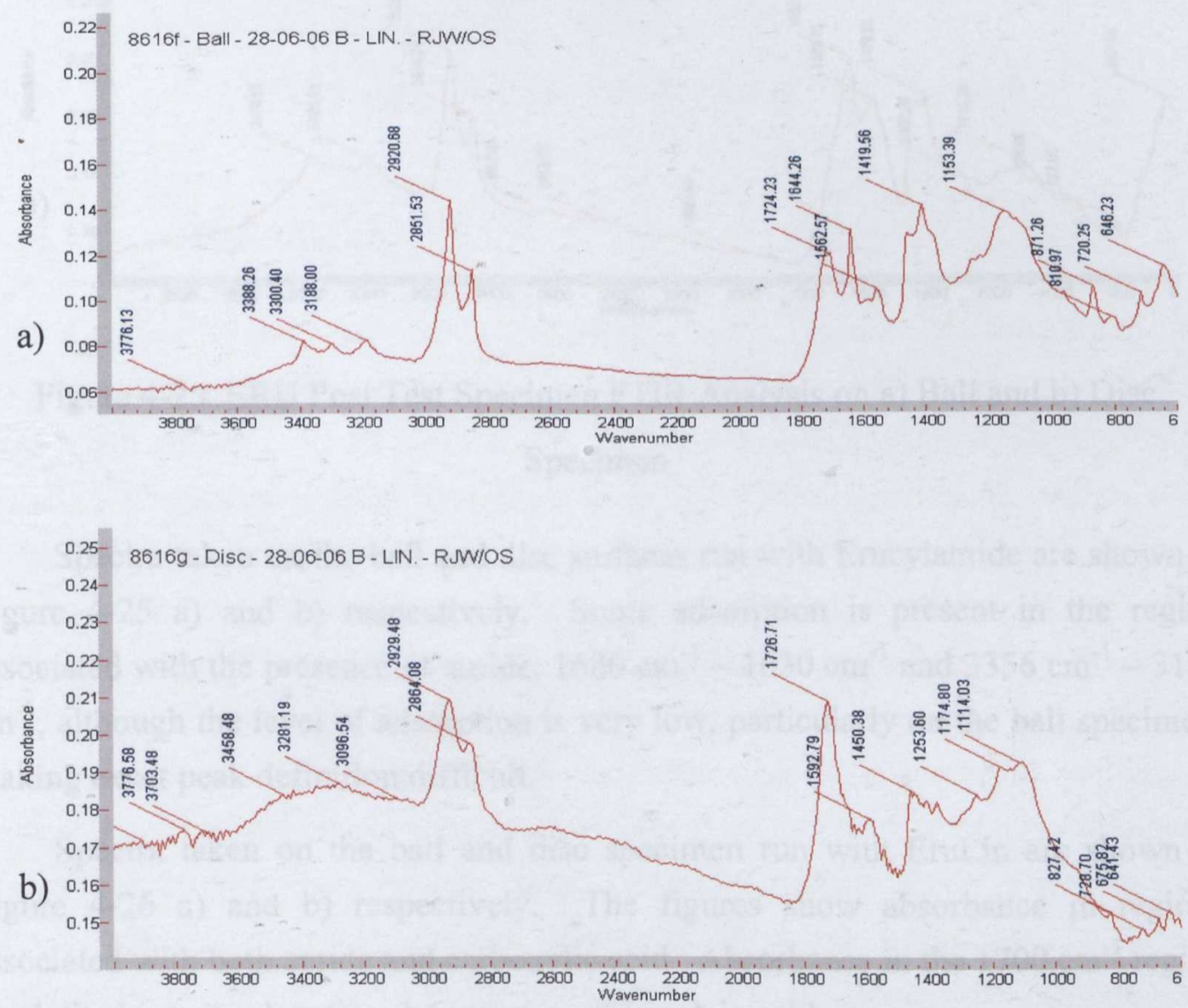

Figure 4-24 LIN Post Test FTIR Analysis on a) Ball and b) Disc Specimen 
Spectra taken on the ball and disc surfaces run with Linoleic acid are shown in Figure 4-24 a) and b) respectively. The figures show a substantial absorbance in the $1700 \mathrm{~cm}^{-1}$ region indicating the presence of carboxylic acid, in addition to the usual peaks associated with the base oil. This absorption is thought to be associated with the presence of Linoleic acid on the surface of the specimens.
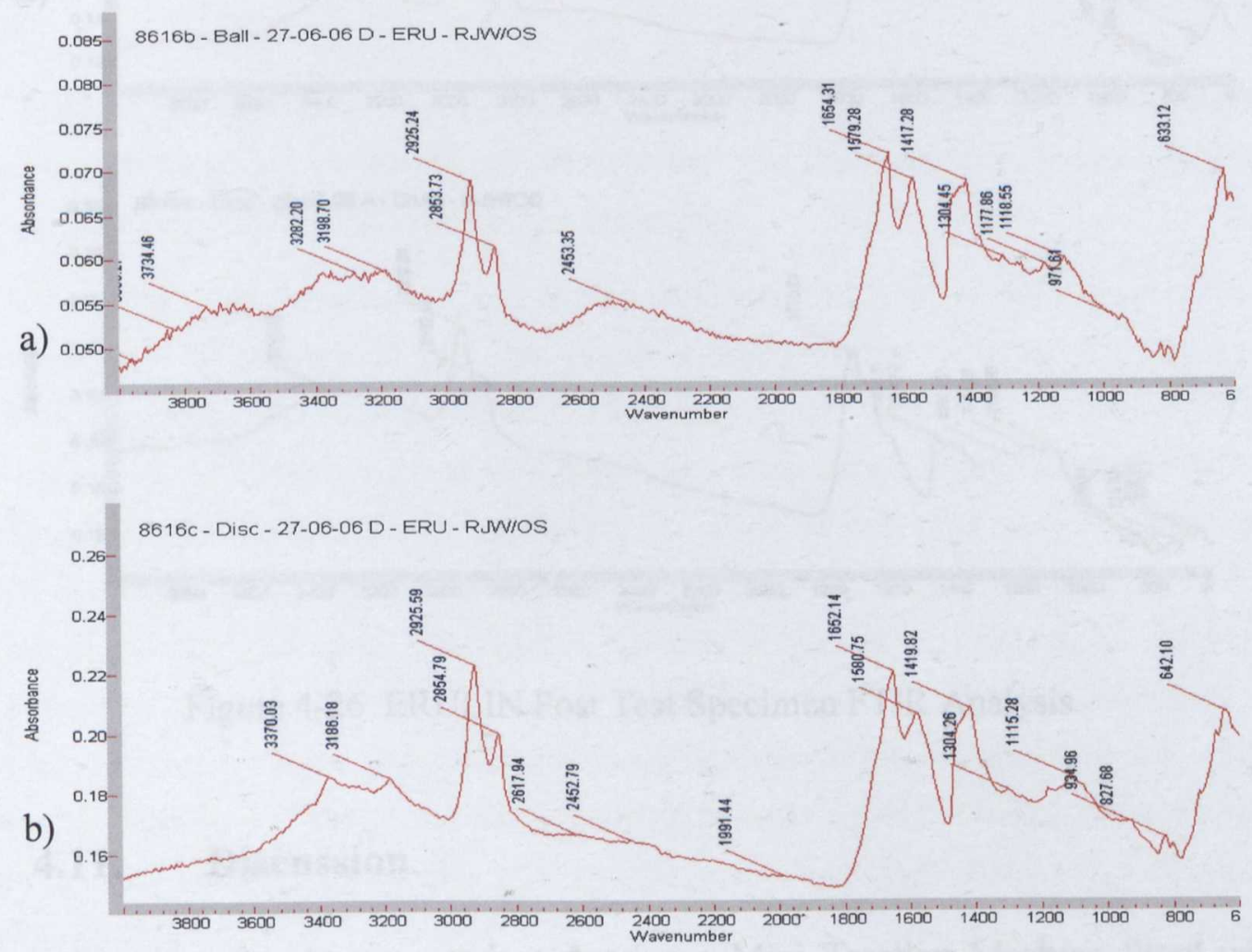

Figure 4-25 ERU Post Test Specimen FTIR Analysis on a) Ball and b) Disc

\section{Specimen}

Spectra taken on the ball and disc surfaces run with Erucylamide are shown in Figure 4-25 a) and b) respectively. Some adsorption is present in the region associated with the presence of amide, $1680 \mathrm{~cm}^{-1}-1630 \mathrm{~cm}^{-1}$ and $3356 \mathrm{~cm}^{-1}-3186$ $\mathrm{cm}^{-1}$, although the level of adsorption is very low, particularly on the ball specimen, making exact peak definition difficult.

Spectra taken on the ball and disc specimen run with EruLin are shown in Figure 4-26 a) and b) respectively. The figures show absorbance in regions associated with both amide and carboxylic acid. Absorbance in the $1700 \mathrm{~cm}^{-1}$ region is relatively strong denoting the presence of Linoleic acid. 

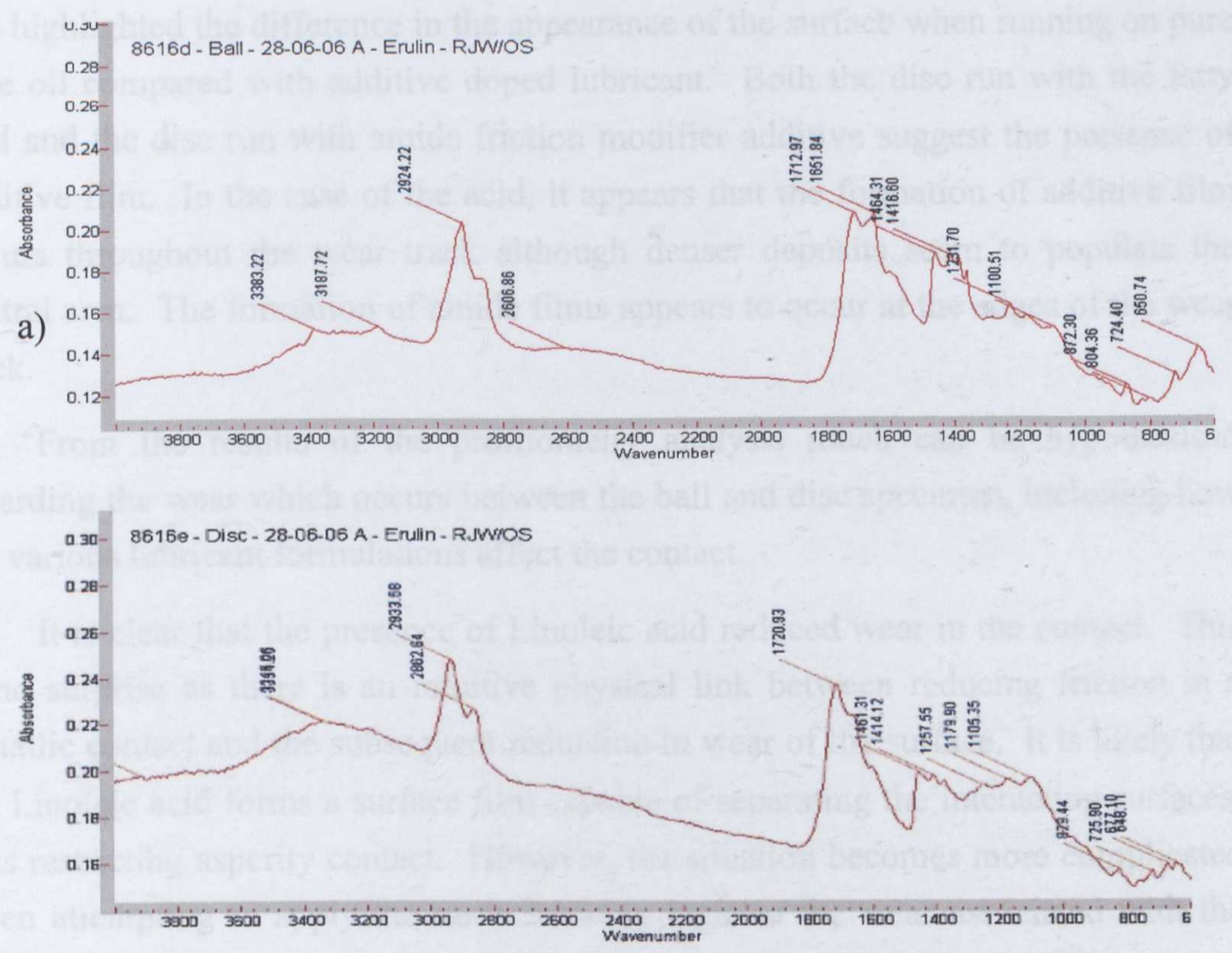

Figure 4-26 ERULIN Post Test Specimen FTIR Analysis

\subsection{Discussion}

A series of tests was conducted using a Mini Traction Machine fitted with bespoke components to simulate engine conditions. The tests were conducted for three main reasons. First, data taken from the machine when operating in the boundary lubrication regime provides a basis for a trend comparison of data taken from the High Frequency Reciprocating Tribometer tests reported in Chapter 3. Second, detailing the performance of the candidate additives across the mixed lubrication regime is important when hypothesising its effectiveness in the engine environment. Finally, the machine provides the perfect opportunity to create some additive films on samples suitable for sophisticated surface analysis techniques. Surface analysis techniques employed included optical microscopy, profilometry, scanning electron microscopy and energy dispersive $\mathrm{x}$-ray analysis as well as $\mathrm{x}$-ray photon spectroscopy and Fourier transform infra-red techniques. Through consideration of results taken in the portfolio of surface analysis with the data taken during the MTM test itself it is possible to begin to understand the mechanism of friction reduction as a result of the application of friction modifier additive. 
Optical microscopy gave a good overview of the surface of the disc specimen, and highlighted the difference in the appearance of the surface when running on pure base oil compared with additive doped lubricant. Both the disc run with the fatty acid and the disc run with amide friction modifier additive suggest the presence of additive film. In the case of the acid, it appears that the formation of additive film occurs throughout the wear track although denser deposits seem to populate the central area. The formation of amide films appears to occur at the edges of the wear track.

From the results of the profilometry analysis much can be hypothesised regarding the wear which occurs between the ball and disc specimen, including how the various lubricant formulations affect the contact.

It is clear that the presence of Linoleic acid reduced wear in the contact. This is no surprise as there is an intuitive physical link between reducing friction in a metallic contact and the subsequent reduction in wear of the surface. It is likely that the Linoleic acid forms a surface film capable of separating the interacting surfaces, thus restricting asperity contact. However, the situation becomes more complicated when attempting to apply the same intuitive logic to the wear associated with the action of the Erucylamide. The additive reduces friction in the contact, but appears to increase wear. Although it is also hypothesised that Erucylamide interposes a surface film capable of separating surfaces from extreme asperity contact, thus reducing friction, it is suggested that the amide reaction with the metal is one severe enough to remove iron molecules from the surface. The action of the amide appears corrosive.

The profilometry results allow us to further understand the synergistic action of the acid and amide blend tested in the ERULIN test, Figure 4-18. Having an average wear area close to that expected when operating a lubricated contact with only Linoleic acid in base oil, it is suggested that the formation of the acid film controls the wear behaviour.

The analysis of both ball and disc surfaces using SEM/EDX techniques did not give any information regarding the presence of any additive film on either surface due to the depth of the analysis conducted. This result tells us that any film which may be present on the surface must be thin. The images and surface maps taken on the specimens do give us further information regarding wear. Figure 4-19 and Figure 4-20 clearly show that wear mainly occurs on the softer disc specimen rather than the hard steel ball. Transport of substrate material as a result of the contact geometry appears to be radially from the inside to the outside of the disc, resulting in deposits. This information clarifies data from the profilometry analysis which suggests the peaks at the outside of the track might be thick deposits of additive. 
The XPS data tell us a great deal about the situation $3-10 \mathrm{~nm}$ below the surface of the specimen. This is the exact location in which thin organic friction modifier films might form, and as such the data taken from the XPS technique carries a lot of weight in investigations such as this. However, interpretation of the data taken from the XPS analysis is a skilful art, greatly guided by knowledge and experience. As such, the views discussed in the following paragraphs are the views and thoughts of the author and are by no means definitive.

The reduced detectable iron $(\mathrm{Fe} 2 \mathrm{p})$ concentration found on discs where friction modifier additive was used suggests the immediate surface of the specimen is covered with molecules consisting of elements other than iron. This effect is particularly pronounced where Linoleic acid was the friction modifier used in the lubricant, suggesting its formation is in thicker films, or that its surface coverage is more complete than that of the amide additive. Given the hydrocarbon chain length of both the acid and the amide is greater than the depth of the XPS analysis, should a film exist in a relatively normal fashion to the surface, theoretically it would completely isolate the metal substrate from analysis. Where both acid and amide are present, the amount of Fe2p detected reflects more the situation where only acid was used, and suggests film formation is dominated by acid.

Analysis of the O1s concentrations suggests that more is detected on the specimen tested with base oil alone than where additive is present. The Ols peak probably manifests itself as an oxide layer in the case of the detection associated on the disc for base oil only, but subtle changes in the binding energy associated with the peak where acid additive was used may suggest some molecular form change. This theory is further validated when looking at the binding energy associated with the $01 \mathrm{~s}$ peak detected where amide additive was used. The binding energy is almost exactly what would be expected when no oxygen containing additive was used. Interestingly where both acid and amide are present the binding energies again suggest that the acid formation dominates, as a slight but significant change is seen. However, the synergistic effect of the additives appears to reduce the amount of detectable $\mathrm{O} 1 \mathrm{~s}$ on the surface. As noted before, the concentrations represent a proportion of the species present on the total surface, and as such it is not generally plausible to compare between test series, however, in this case the lack of oxygen combined with the increase of $\mathrm{C} 1 \mathrm{~s}$ detection suggests bulk comparison is of interest. It may be the case that a thicker or more densely coherent film is formed where both additives are available to react with the surface.

Inspection of the data associated with the N1s peaks suggests the presence of nitrogen on the surface of the disc substrate in all cases, except for on the wear track of the disc run with Linoleic acid. The presence of nitrogen is detectable at trace 
levels in the test where only pure XHVI-8.2 ${ }^{\mathrm{TM}}$ (Shell) was used, which lacked amide or nitrogen based species. This suggests there may have been some contamination in the test caused by the presence of amide left behind in the lubricant reservoir from previous tests. Where Linoleic acid was used as a friction modifier the presence of N1s was minute, suggesting either no contamination was present in the test, or the long chain acid molecule masked its detection through the interposition of its long hydrocarbon chain. Interestingly, where Erucylamide was the friction modifier a greater concentration of N1s is detectable on the surface, which when combined with a slight shift in the binding energy may suggest the detected nitrogen was in a different form. Where both amide and acid are present a high concentration of N1s is recorded suggesting the detection of the amide additive film. In addition the presence of $\mathrm{Na}$ in both tests conducted with the amide suggests some impurity in the Erucylamide stock. This is not unexpected as in the process through which Erucylamide is created, saponification, sodium hydroxide is often used as a catalyst which can result in trace amounts of $\mathrm{Na}$.

It is clear from inspection of the data that there are some differences between analyses conducted on and off the wear track. However, these differences do not appear to be as substantial when comparing between data sets. It is worth noting again at this point that comparison between data sets is one which should be conducted with care as the measured concentration of each species is dependent on its peers also present on the area of surface under analysis, the totals of which sum to $100 \%$. In addition, the surface topography of the wear track area differs greatly from that outside. Given the friction modifier additives are polar species, both the amount and state of the surface available for reaction is likely to be of great importance. A typical trend suggests the increased presence of hydrocarbon film outside the wear track. However, data relating to the Erucylamide measurement suggests the inverse of this relationship. This could of course be the product of the change in the proportion of $\mathrm{O} 1 \mathrm{~s}$ species measured at the same time, or equally the reciprocal could be the case. Generally then, it is possible to hypothesise the conclusion that the films do not necessarily manifest themselves as 'tribofilms', films that form as a result of contact mechanics, but as independent surface films.

It was stated earlier that the $\mathrm{C} 1 \mathrm{~s}$ detection was almost completely associated with $\mathrm{C}-\mathrm{C}$ hydrocarbon groups, with a low level of non-specific $\mathrm{C}-\mathrm{O}$ bonding found (section 4.9). Given this information, it is possible that a lot of the measured signal was due to adsorbed test fluid residue, especially outside the wear track. However, analysis of the detected concentrations reveals there was more $\mathrm{C} 1 \mathrm{~s}$ detected on specimens tested with friction modifier than that tested without. The surface depth under analysis is likely to consist of the hydrocarbon tails of the polar acid 
molecules, thus increasing the intensity of the C1s peak measurement. Presence of C1s is particularly pronounced where acid based friction modifier was used, suggesting there may be a greater quantity of $\mathrm{C}-\mathrm{C}$ bonds on the specimen surface. This hypothesis corroborates the suggestion made earlier that Linoleic acid friction modifier forms either in thicker films, or more probably, films with more complete surface coverage than those formed by the amide. Interestingly, the disc subjected to both acid and amide exhibits the greatest proportion of $\mathrm{C} 1 \mathrm{~s}$ which may suggest the thickest, or most dense, hydrocarbon film is formed as a result of the synergistic relationship between the species.

It is worth noting at this point that the analyses conducted provides no evidence for the presence of robust chemically bonded surface films. However, qualitative analysis guides the hypothesis stating that organic friction modifiers do interact with the metallic specimen surfaces resulting in the creation of low friction contacts.

FTIR analysis of the ball and disc specimens shows the technique is capable of detecting and determining between the films formed on the samples run with different additive chemistries. However, it is difficult to identify specific compounds, since the spectra are complex and not very well resolved.

Detectable levels of additive chemistry on the surfaces of both ball and disc confirm the findings of the XPS analysis which suggested some friction modifier film is attached to the surface disc specimens, but go further to show some friction modifier presence on the ball speeimens. However, the resolution of the recorded spectra is not high enough to determine the state of the attachment on either ball or disc specimen.

Where acid and amide are run separately, results suggest that the carboxylic acid, Linoleic acid, surface film might be more complete than that of the amide, Erucylamide. Relatively high levels of adsorption in the carbonyl region were seen where Linoleic acid was used compared with trace adsorptions seen in the amide region, where Erucylamide was used. Ball and disc specimens run with EruLin show a reasonably strong presence of carboxylic acid, Linoleic acid, and weak presence of Erucylamide. When similar FTIR analyses were conducted on cylinder liner specimens run in the TE-77 reciprocator in section 3.6.5, the reverse of this finding was seen. Where Erucylamide was used, a strong adsorption in the region associated with amide formation was clearly detectable on both piston ring and cylinder liner specimen. Where Linoleic acid was used there was little evidence in the absorbance spectra to suggest the presence of carboxylic acid. Where EruLin was tested in the TE-77, evidence of amide formation was seen with no evidence of carboxylic acid formation at all. It is hypothesised that the discontinuity between 
FTIR analyses conducted in section 3.6 .5 and section 4.10 is a result of many factors, the most important and influential of which are listed below:

- The specimens were subjected to different test conditions. In the TE-77 tests the conditions were particularly arduous and operation was always in the boundary lubrication regime, under pure sliding. In the MTM tests the conditions cycled between elastohydrodynamic, mixed and the boundary lubrication regime, in both sliding and rolling.

- The temperatures were conducted at different temperatures. In the TE77 tests the liner surface temperature was controlled at $110^{\circ} \mathrm{C}$. In the MTM tests the temperature was controlled at $125^{\circ} \mathrm{C}$.

- The base oil used in each test was different. In the TE-77 tests were conducted with XHVI-4TM. In the MTM the tests were conducted with a more viscous XHVI-8.2 $2^{\mathrm{TM}}$ base oil.

- The surface roughnesses of the specimen surfaces were considerably different. In the TE-77 machine the cylinder liner specimen is finished with a cross-hatch hone giving an $\mathrm{Ra}$ of approximately $0.83 \mu \mathrm{m}$. The disc specimen was finished to an $\mathrm{Ra}$ of approximately $0.01 \mu \mathrm{m}$, to permit surface analysis.

The information provided through surface analysis of test specimens describes both the composition of the surface films, and gives an indication as to their size, but does not describe the way in which they form, or under what conditions they are stable, or even effective. To further understand the friction modifier films and their ability to reduce friction in the contact it is necessary to analyse the coefficient of friction data taken from the MTM tests themselves.

It is clear from all previous results that lubricant containing Erucylamide is thought to act as a friction modifier in a distinctly different manner from that of Linoleic acid. The acid is thought to form thick films in the mixed regime which have the capability to reduce friction when compared to that of base oil alone, but Erucylamide appears to create films which are more difficult to shear down to a film of lower shear strength, films which maintain dominance over the frictional characteristics of the contact well into the low speed region. However, again at low speeds promoting boundary lubrication a marked decrease in friction coefficient indicates the reduction of the thick film to that of a thin solid-like film with lower shear strength. The data suggests more tenacious films are formed in metal/amide contacts than in metal/fatty acid contacts, as this thinning process occurs under more arduous conditions where amide is used. In addition, there appears to be little time related effect associated with the formation of amide films. Between the first run, 
and any proceeding, little changes. This may indicate the recurring formation and removal of films on the surface, as opposed to the acid films formation which appears to be one in which a monolayer attaches to the surface, and allows further layers to build on top.

The synergistic action of the amide and the acid suggests, from a frictional characteristic point of view, that the combination of chemical compounds leads to lower friction in the contact throughout the test. The acid dominates initial film formation on the metal surfaces resulting in low friction in the mixed lubrication regime, and well into that of the boundary. At low speeds the amide film takes advantage of the weak attachment of the acid, and subsequent partial removal from the surface, by forming on the clean metal surface. This partial film is not detached as a result of the increasingly arduous conditions. With time it seems that the acid films continual formation serves to create ever thicker films with even lower shear strengths resulting in further reduced friction in the contact. In fact the growth of these films appears to promote the onset of elastohydrodynamic/hydrodynamic behaviour in the contact, indicated by the rise in coefficient of friction displayed in the high speed region. In contrast to the acid, the amide exhibits little time related behaviour, although some evidence suggests it too promotes the transition from the mixed lubrication regime to that of the boundary. The blend of the two additives appears to have beneficial synergistic effects resulting in the lowest friction operation throughout the complete curve. Final coefficients of friction are slightly elevated compared with those measured in run 1, and are perhaps a result of modifications to the surface caused by slight wear of the samples.

\subsection{Summary}

In this chapter a set of Mini Traction Machine tests are reported, whose purpose was to both investigate the performance of novel organic friction modifiers over the mixed and boundary regime, and to create some specimens on which surface films suitable for detailed surface analysis have been formed. The chapter describes the results, analysis and interpreted meaning of the various surface analysis results. This chapter is of great significance in the investigation of novel organic compounds for use as gasoline friction modifiers as a substantial amount has been learnt through the detailed analysis of the portfolio of data. On consideration of all data presented in this chapter, the following summary can be made.

- The results of the MTM tests suggest the addition of the organic friction modifiers to base oil can reduce boundary friction by more than half. The most effective boundary friction modifier was ERULIN, a 
lubricant blend containing both $1 \mathrm{wt} \%$ Linoleic acid and $1 \mathrm{wt} \%$ Erucylamide. A typical value of boundary friction coefficient measured in this test was 0.08 for ERULIN.

- Both acid and amide friction modifiers exhibited some friction reduction capability in the mixed lubrication regime, the former appearing the most effective. When combined in a lubricant blend, the acid appears to dominate film formation over the amide in the mixed regime.

- When operating in the mixed lubrication regime, both acid and amide are thought to form thick films on the metal substrate surfaces which serve to act like a viscous film. The frictional characteristics of the films are believed to be dominated by the shearing of layers of additive molecules between themselves.

- In pure boundary lubrication conditions, the viscous films which dominate the contact in the mixed lubrication regime are thinned down to a thin solid-like film of low shear strength. Thick films created by amide layers appear more difficult to thin down than those created by acids, as they maintain dominance over the frictional characteristics of the contact well into the region where pure boundary lubrication should dominate.

- In contrast to the acid film, the amide additive exhibits little time related effects in film formation. This possibly suggests the recurring formation and removal of films on the surface, as opposed to the acid film formation which appears to be one in which a monolayer attaches to the surface, and further layers build on top.

- When both acid and amide are employed in the lubricant blend, the amide appears to be capable of complementing the action of the acid by allowing the acid to dominate film formation over the conditions at which it excels at reducing boundary friction, and by assisting in friction reduction at conditions where acid films can be removed.

- The presence of fatty acid in a lubricant appears to reduce wear on the running surfaces, whereas the presence of amide appears to increase wear of the surfaces. It is hypothesised that the amide reaction with the substrate metal is one severe enough to remove iron molecules from the surface. The action of the amide friction modifier appears corrosive. 
- Where both Linoleic acid and Erucylamide are present, the wear profile results suggest the Linoleic acid has had a dominant effect over the amide as the wear remains low.

- XPS analysis of the disc surfaces proved inconclusive, but highlighted the presence of relatively neutral $\mathrm{N}$ species and $\mathrm{C}-\mathrm{O}$ bonding which is thought to be evidence of amide and acid films respectively. Given the level of cleaning the specimens were subject to prior to the XPS analysis, it seems wrong to suggest these films were merely physically attached to the surface, yet there is no evidence to suggest the presence of dense or robust chemical films.

- Films formed by Erucylamide and Linoleic acid do not necessarily form 'tribofilms', which are films formed as a result of contact mechanics, but form as independent surface films.

- Where both amide and acid were present in the lubricant formulation qualitative analysis of surface concentrations and species binding energies detected by XPS analysis suggests the acid formation dominates the film. 


\section{Novel Fuel Formulation Engine Tests}

Previous chapters have described the investigation into the hypothesis stating engine friction can be reduced through the application of gasoline administered friction modifiers. Chapter 2 described lubricant sampling tests which not only validated the hypothesis that additive molecules can be administered to both the piston top ring groove and the cylinder liner oil film via gasoline, but that the lubrication system at these locations permits additive accumulation. Chapter 3 gave explanation of the results of novel additive screening tests conducted on the Cameron Plint TE-77 bench-top tribometer, where tests were conducted in the boundary lubrication regime to simulate the arduous lubrication condition at the top dead centre location. Analysis of data allowed the additives to be categorised in terms of performance characteristics, identifying those thought to be most effective in the engine environment. Chapter 4 described the testing of additives using the Mini Traction Machine, scoping the performance of selected high performing additives in the elastohydrodynamic, mixed and boundary lubrication regimes. Following on from the findings of these chapters, this chapter describes a series of engine tests where the effects of gasoline doped with friction modifier additive are tested in a firing engine. Engine tests conducted fall into two distinct categories. First, a series of scouting tests were conducted where engine performance was analysed when running on standard or FM gasoline. Second, a series of tests were conducted where piston assembly friction was measured directly using the Instantaneous Mean Effective Pressure (IMEP) method when running on standard or Friction Modified (FM) gasoline, allowing the determination of areas within the piston cycle where the novel friction modifiers perform best.

\subsection{Engine Testing}

The Ricardo Hydra gasoline engine presented in Chapter 2 was used for all engine tests described in this chapter.

Having seen the promising effects of the novel friction modifier additives in the bench-top tribometer, it was necessary to assess their performance in an engine. Although the effects of the novel friction modifier additives were quite dramatic in the tribometer tests, their impact in the engine were expected to be less. For this reason engine tests were structured accordingly. The first set of tests served to scout the performance of the FM gasoline and were designed to look for an increase in the dynamometer measured brake torque when the fuel supply was switched from 
standard to FM gasoline. The second set of tests involved instrumenting the engine with bespoke components which facilitate the measurement of piston assembly friction through the application of the IMEP method. It was thought that if there was little difference between the dynamometer measured torque when running on Standard and FM gasoline, there was little point in instrumenting the engine for IMEP friction measurements, a task which is not considered trivial.

\subsubsection{Test Fluids}

Despite the promising effects seen in previous chapters of the 'ERULIN' friction modifier blend, it was not tested in the engine due to problems encountered with blending. When blending the Erucylamide component with base oil, as was the case for previous bench-top tribometer based tests, the mixture was heated to temperatures in excess of $79^{\circ} \mathrm{C}$ to allow the additive to exceed its melting point. As this series of tests required the amide to be blended with gasoline, heating the mixture was not an option. Various methods of blending the amide with gasoline were conceived, but were considered too dangerous to attempt in-house. For this reason, only Linoleic acid was tested in the engine, its form being liquid at room temperature facilitating safe and easy blending in-house. The FM gasoline was added to a standard unleaded (ULG) 95 RON gasoline without an additive package, which was excluded to limit complications. Details of the two fuels used in the following tests are given below.

\begin{tabular}{ccc}
\hline Name & Base Fuel & Additive Package \\
\hline STD & $100 \%$ Standard ULG 95 & None \\
\hline FM & 98 wt\% Standard ULG 95 & 2 wt\% Linoleic Acid \\
\hline
\end{tabular}

Table 5-1 Fuels Used in Engine Tests

For the crankcase oil it was decided to use additive free XHVI-8.2 ${ }^{\mathrm{TM}}$ (Shell) base oil. This type of lubricant was used in all tests conducted in this chapter. As was the case with the gasoline, additives were excluded to limit complications. However, it is important to note that for this set of tests the valve train was fed from a second sump containing a Shell SAE $5 \mathrm{~W}-30$ fully formulated lubricant. This focussed the effects of the fuel administered friction modifier to the crankcase side of the engine, principally to the piston assembly. It was also not considered desirable to have XHVI-8.2 ${ }^{\mathrm{TM}}$ and friction modifier lubricating the valve train. As such, any measured effect is only the result of friction modifier effects on piston assembly friction. 


\subsubsection{Running-In}

Prior to testing, a new piston assembly featuring a chrome coated piston ring pack, as described in section 3.3.4, was run-in against a new cylinder liner equipped with a TDC near-surface thermocouple. The standard running-in procedure was not used for these specimens, as running-in took place with additive free fluids. providing minimal protection to component surfaces. The running-in procedure was designed to ensure components were suitably run-in for the conditions under which the following tests would be conducted. It is known that the IMEP friction measurement tests have greatest resolution at speeds not in excess of $2000 \mathrm{rpm}$, a speed which also protects against component failure (Mufti, 2004). Given this information it was decided that tests would not be conducted beyond a maximum condition of $2000 \mathrm{rpm}$ and full load. For this reason running-in beyond this condition was not thought to be necessary. In addition, running-in at excessive speed and loads with unformulated oils is dangerous and can result in catastrophic engine failure. The running-in procedure followed is detailed in Table 5-2.

\begin{tabular}{ccccc}
\hline Step & Duration (Hrs) & Speed (rpm) & Load $(\%$ of full $)$ & Temp $\left({ }^{\circ} \mathrm{C}\right)$ : Oil/Coolant \\
\hline 1 & 6 & 1500 & 50 & $90 / 90$ \\
\hline 2 & 6 & 2000 & 50 & $90 / 90$ \\
\hline 3 & 10 & 2000 & 100 & $90 / 90$ \\
\hline
\end{tabular}

Table 5-2 Running-in Procédure

\subsubsection{Engine Performance Testing}

The main aim of the Engine Performance tests was to investigate the performance related effects of fuel administered friction modifier on gasoline engine brake torque. Tests were designed to accurately assess the difference in engine brake torque as a result of changing from STD gasoline to FM gasoline without disruption to engine operation. To ensure the measurement of engine torque was as accurate as possible the dynamometer was calibrated prior to testing, and checked post-test. To do this a series of weights were hung on the calibration arms which extend from the side of the main unit. Through applying a series of static loads and measuring the associated torque, the calibration of the machine could be checked against the manufacturer's guidelines. The engine dynamometer calibration result is shown in Figure 5-1.

To accurately measure the brake torque a bespoke LabVIEW program was developed. As well as monitoring various fluid temperatures around the engine, the 
program simultaneously monitored the thrust side near-surface cylinder liner temperature at TDC and the signal from the exhaust gas oxygen sensor mounted in the exhaust down-pipe. Measurements were taken and recorded using the LabVIEW system at 20 second intervals.

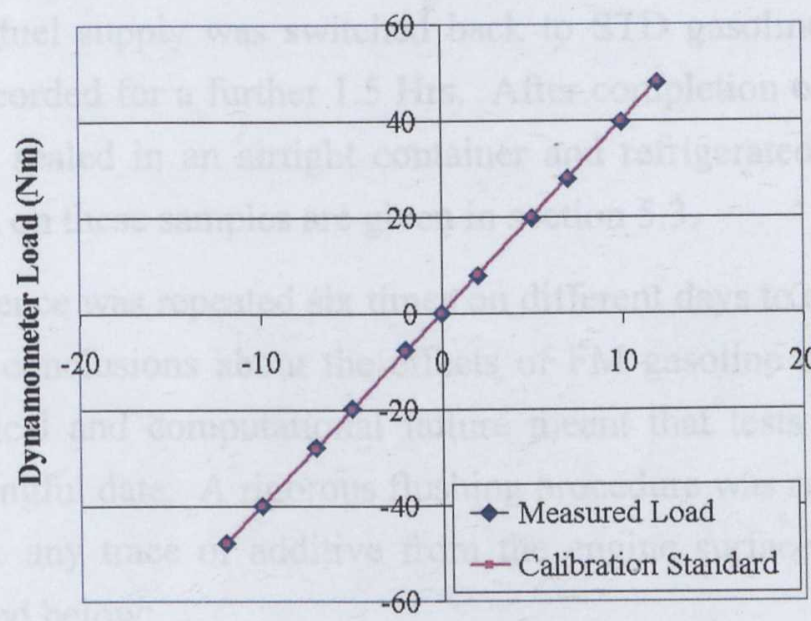

Static Load (Kg)

Figure 5-1 Engine Dynamometer Calibration

The test programme began by running the engine on STD gasoline for approximately 2 Hrs until thermal equilibrium had been reached and engine operation stabilised. From this point no further alteration to engine operating condition was allowed and engine controls were locked. The engine test conditions for the performance tests are shown in Table 5-3.

\begin{tabular}{cc}
\hline Parameter & Condition \\
\hline Speed & $2000 \mathrm{rpm}$ \\
\hline Load & $25 \mathrm{Nm}(80 \%$ of full $)$ \\
\hline Mixture & Stoichiometric \\
\hline Ignition & $11^{\circ} \mathrm{BTDC}$ \\
\hline Coolant Temperature & $90^{\circ} \mathrm{C}($ Controlled $)$ \\
\hline Crank Sump Temperature & $90^{\circ} \mathrm{C}($ Controlled $)$ \\
\hline Cam Sump Temperature & $78^{\circ} \mathrm{C}$ \\
\hline Air Inlet Temperature & $41^{\circ} \mathrm{C}$ \\
\hline Fuel Inlet Temperature & $25^{\circ} \mathrm{C}$ \\
\hline
\end{tabular}


Once running at thermal equilibrium conditions the test began with a baseline brake torque measurement taken for a period of two hours. The fuel supply was then changed to one containing FM gasoline without disruption to engine operation. A brake torque measurement was recorded for a 3 hour period with the engine running on gasoline doped with friction modifier. After this period, again without disruption to the engine, the fuel supply was switched back to STD gasoline. Engine brake torque was then recorded for a further 1.5 Hrs. After completion of the test a sump sample was taken, sealed in an airtight container and refrigerated. Details of the analyses conducted on these samples are given in section 5.3.

The test sequence was repeated six times on different days to create a firm base on which to form conclusions about the effects of FM gasoline on engine torque. However, mechanical and computational failure meant that tests 2 and 5 did not produce any meaningful data. A rigorous flushing procedure was employed between all tests to remove any trace of additive from the engine surfaces. This flushing procedure is detailed below:

\begin{tabular}{cc}
\hline Step & Action \\
\hline 1 & Change engine oil. \\
& Drain oil filter and replace. \\
\hline 2 & Run engine on STD gasoline for $1 \mathrm{Hr}$. \\
\hline 3 & Change engine oil. \\
& Change oil filter. \\
\hline
\end{tabular}

Table 5-4 Engine Flushing Procedure

It would have been beneficial to conduct a test where the engine was run on STD gasoline for a full day, to map change in power output with only time as a variable. However, data collected for the sixth repeat test showed signs indicating the engine was not performing well. Performance had decreased to such an extent that an engine strip-down and inspection was conducted, the results of which are displayed in Figure 5-2. The inspection revealed that large amounts of varnish and deposits had formed on the surfaces of all the key components, primarily a result of running tests without any additive package. The cylinder liner walls were thick with varnish, to the extent that it was unlikely that any significant amount of metal was exposed to react with the friction modifier additive (Figure 5-2a). The piston crown was black with a thick covering layer of burnt deposits, particularly on the intake side, shown on the right hand side of Figure 5-2b. Both the intake and exhaust 
valves were coated with deposits, to such an extent that the valve seat was obstructed, thus compromising the seal (Figure 5-2c). The piston itself was also coated with a thick layer of varnish and deposits (Figure 5-2d). The movement of the top compression ring appeared to be slightly restricted due to the deposits found in the ring groove area. The engine was considered to be in such a state that if further testing was conducted, failure would have surely resulted from piston ring seizure. It was for this reason that subsequent testing was not conducted and the engine was stripped down and cleaned thoroughly.

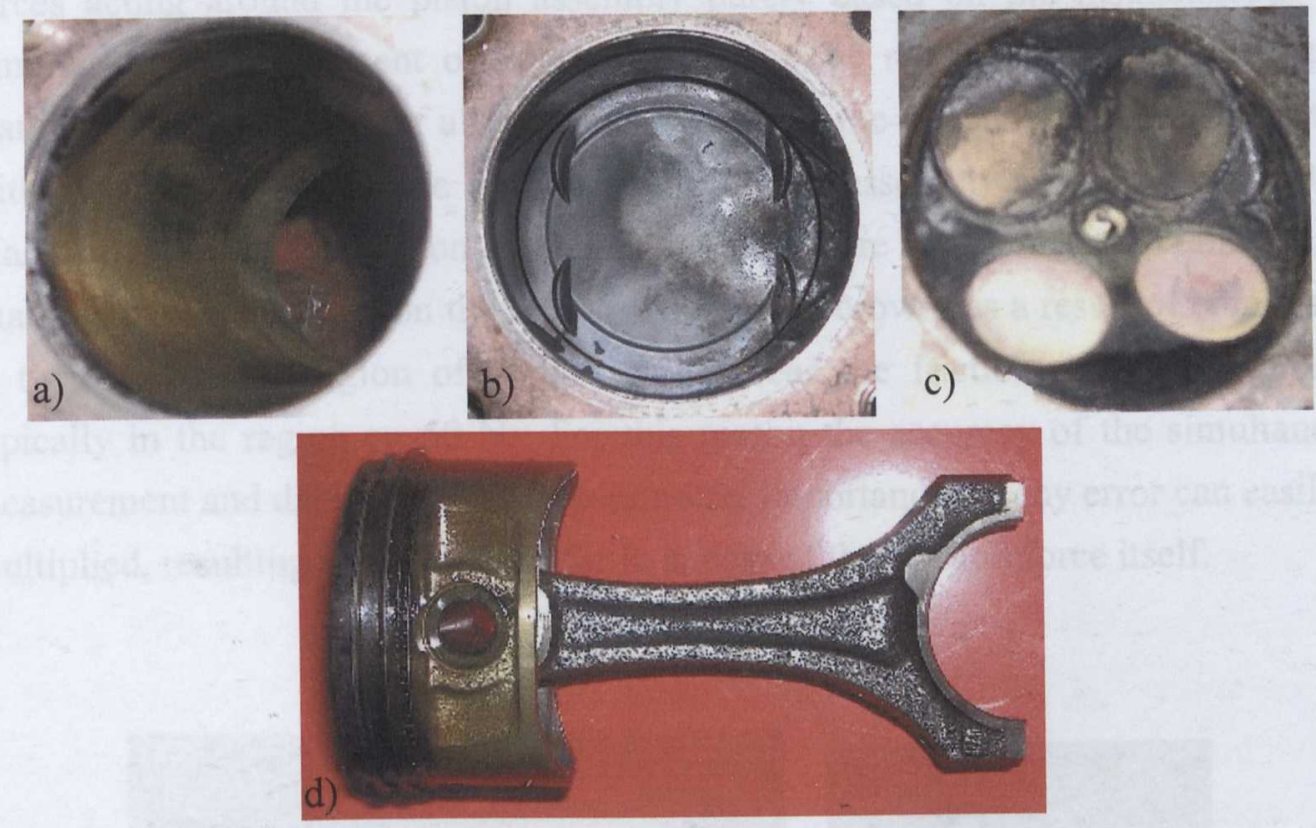

Figure 5-2 Inspection of Components After Sixth Engine Test: a) Cylinder Bore, b) Piston Crown, c) Valves, d) Piston and Connecting Rod.

\subsection{Engine Friction Testing}

The main aim of the engine friction tests was to investigate the performance related effects of fuel administered friction modifier on measured gasoline engine friction. To do this the engine was stripped down to its component parts, cleaned with acetone and rebuilt with the bespoke apparatus needed to make the IMEP total engine friction measurement possible, Figure 5-3. The IMEP total engine friction measurement system was created by Riaz Mufti, and is the subject of his $\mathrm{PhD}$ thesis (Mufti 2004; Mufti and Priest 2005). The work described in this chapter utilises this system as a tool to measure piston assembly friction under differing lubrication conditions, but also goes further to develop the technique. The equation governing the piston assembly friction (f) is given in Equation 6-1. Figure 5-3 gives details of 
the bespoke components and the measurements they make possible with reference to the notation given in Equation 5-1.

$$
f=\left(P_{1}-P_{2}\right) A_{p}-F_{S T G} \cos (\phi)+m_{p} g+F_{c} \cos (\phi)+m_{p} a_{0} \quad \text { Equation 5-1 }
$$

The technique measures physical forces acting on and around the piston assembly, whilst calculating inertia forces which are the result of the assembly motion. Even the calculation which determines the inertial forces is based on information given by a physical measurement taken from the crankshaft encoder, which measures rotation with respect to time. Essentially the formula equates the forces acting around the piston assembly purely based on the instantaneous and simultaneous measurement of key parameters. The resulting equation practically manifests itself as a sum of all the forces acting on the piston assembly as a system, with the unknown being the friction acting on the assembly as a result of motion. Many of the forces acting on the piston assembly are of enormous magnitude, for example the force acting on the area $A_{p}$ (the piston crown) as a result of combustion is typically in the region of $20,000 \mathrm{~N}$, whereas the friction force of interest is typically in the region on $50 \mathrm{~N}$. For this reason the accuracy of the simultaneous measurement and data capture is of paramount importance, as any error can easily be multiplied, resulting in magnitudes far in excess of the friction force itself.
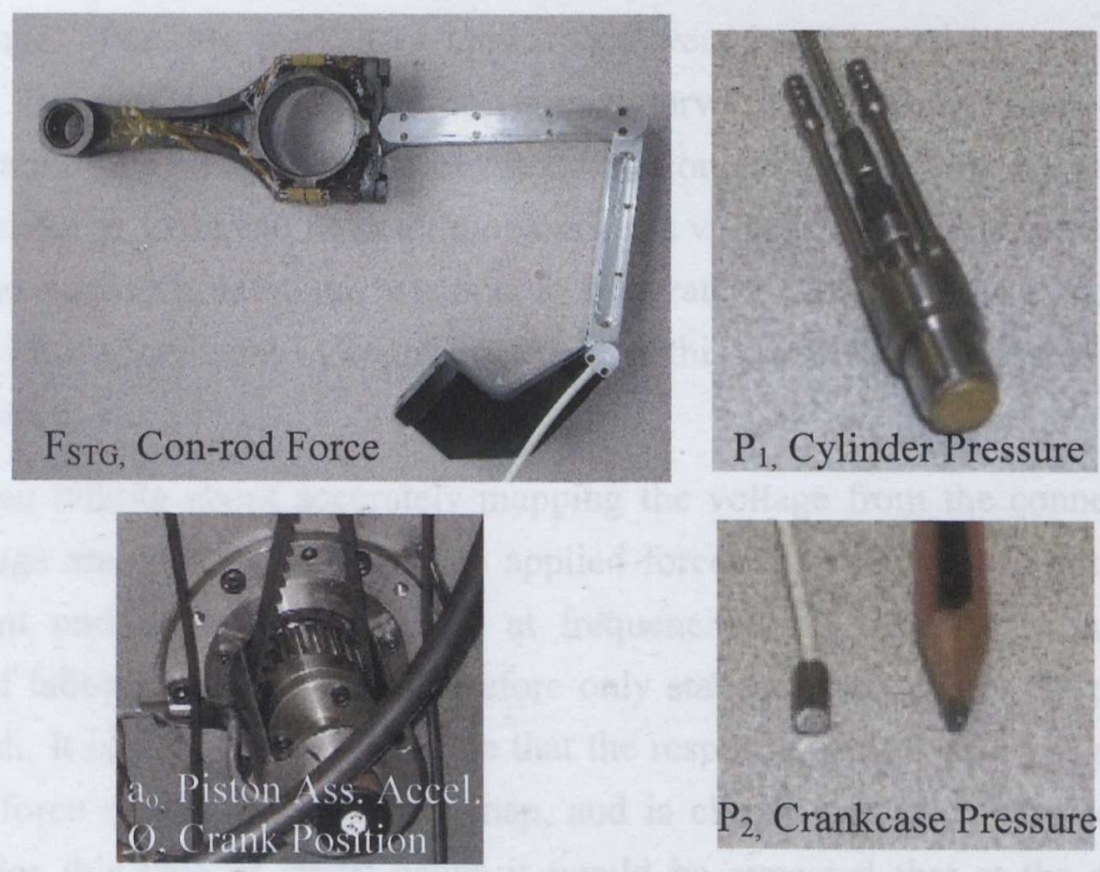

Figure 5-3 Piston Assembly Friction Measurement Apparatus 
Inertia forces are also a key influence on the ability of the system to accurately measure piston assembly friction. To ensure the accuracy of the system was at a maximum, prior to testing all components were weighed in grams to 3 decimal places, and the resultant information included in the program which was used to calculate the component inertia forces.

Despite every effort to ensure maximum accuracy of the system, preliminary testing of the system revealed some error. This error was traced back to the calibration of the strain gauge fitted to the connecting rod. Subsequently the connecting rod was removed from the engine and re-calibrated in the laboratory using a hydraulic Dartec apparatus. The amplified strain gauge voltage signal was recorded against the applied force between the limits of $20000 \mathrm{~N}$ (compression) and $-2000 \mathrm{~N}$ (tension), forces which are typical of those experienced in general operation. Although on a macro scale, the voltage from the strain gauge amplifier can be mapped to the applied force using the simple straight line approximation where the relationship 1997 N/V is valid, it is clear from inspection of Figure 5-4b that this approximation is not valid at low force values. This highlights a problem of magnitude when it is considered that during the cycle of the engine, the connecting rod only experiences forces in the region of $20000 \mathrm{~N}$ once, briefly, after ignition. For the most part of the engine cycle the connecting rod experiences forces in the region of 2000 to $-2000 \mathrm{~N}$, during the three pumping strokes. For this reason it is not valid to use a straight line approximation to calculate the force acting on the strain gauge. For this work, two approaches were investigated to overcome this problem. The first uses a $3^{\text {rd }}$ order polynomial curve fit which maps more closely to the calibration line. The second uses the calibration curve as a 'look-up' table, from which a value of force can be determined given a voltage. The preferred solution is the second approach, although attempts to integrate it with the core program were fraught with complication. Graphs presented in this work were computed using the first approach.

When talking about accurately mapping the voltage from the connecting rod strain gauge amplifier with respect to applied force it is necessary to note that the component undergoes force changes at frequencies and magnitudes outside the bounds of laboratory simulation, therefore only static calibration can be practically conducted. It is also important to note that the response of the strain gauge through the zero force region is difficult to map, and is clearly an area where some error exists. For this type of strain gauge it would be expected that at the zero force condition, a reading of zero volts be measured. From inspection of Figure $5-4 \mathrm{~b}$ it is clear this is not the case, thus it is acknowledged that this offset represents some error in the measurement system. 


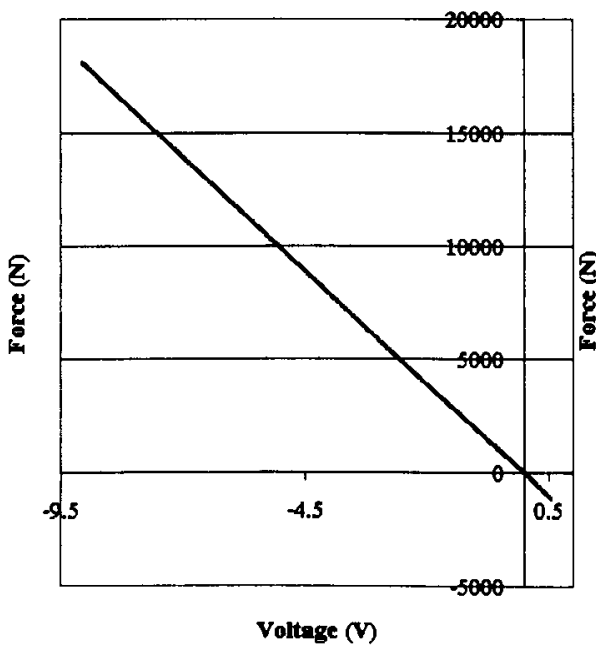

a)

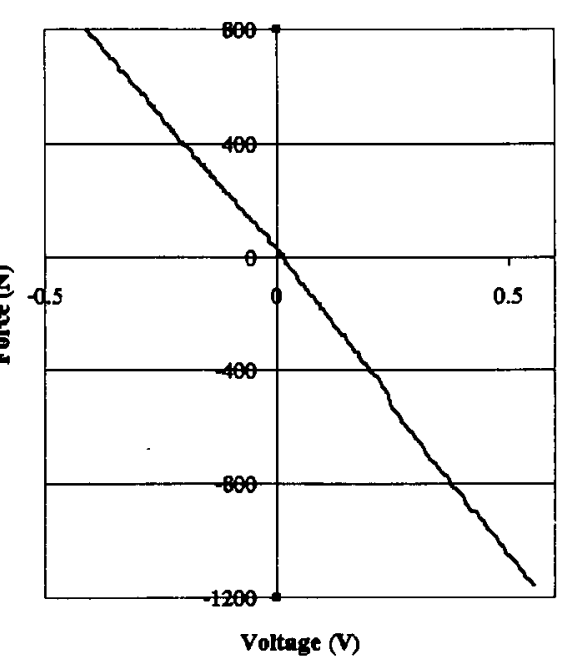

b)

Figure 5-4 Connecting Rod Calibration Graph

\subsubsection{Standard Engine Friction Test Methodology}

Test conditions were selected to simulate typical engine operation at steady state conditions, where engine speed might be relatively low, but load relatively high. However, the test conditions also represent the compromise made as a result of the application of the IMEP friction apparatus. As discussed in previous sections there are particular conditions where the friction data capture system has been shown to capture more meaningful data with less noise. The selected engine test conditions are detailed in Table 5-5.

\begin{tabular}{cc}
\hline Parameter & Condition \\
\hline Speed & $2000 \mathrm{rpm}$ \\
\hline Load & $19 \mathrm{Nm}(60 \%$ of full $)$ \\
\hline Mixture & Stoichiometric \\
\hline Ignition & $11^{\circ} \mathrm{BTDC}$ \\
\hline Coolant Temperature & $90^{\circ} \mathrm{C}($ Controlled $)$ \\
\hline Crank Sump Temperature & $90^{\circ} \mathrm{C}($ Controlled $)$ \\
\hline Cam Sump Temperature & $90^{\circ} \mathrm{C}($ Controlled $)$ \\
\hline Air Inlet Temperature & $41{ }^{\circ} \mathrm{C}$ \\
\hline Fuel Inlet Temperature & $25^{\circ} \mathrm{C}$ \\
\hline
\end{tabular}


To measure the effect of FM gasoline on total engine friction the following test procedure was created. Before the test was started the engine was run for approximately 2 hours on STD gasoline until thermal equilibrium had been established. From this point, engine controls were locked and the test started. A measurement of total engine friction was recorded at 20 minute intervals for $1 \mathrm{Hr}$ using the friction data capture procedure developed by Mufti, detailed in Table 5-6. At this point, and without disruption to the engine, the fuel supply was changed to one containing $2 \mathrm{wt} \%$ Linoleic acid (FM gasoline). Friction data was then captured every 20 minutes for a further hour. After switching back to STD gasoline, friction data captures were again conducted every 20 minutes for a final hour. At this point the flushing procedure detailed in Table 5-4 was carried out to ensure all traces of additive were removed from the engine before the next test.

After the test a sample of sump lubricant was taken in an air tight container and stored in a refrigerator prior to analysis.

\begin{tabular}{cc}
\hline Step & Action \\
\hline & Stop engine. \\
& Cut power to engine controls. \\
Remove timing belt. \\
1 & Zero $\mathrm{P}_{1}, \mathrm{P}_{2}$, Con-rod and Valve train amplifiers. \\
& Refit timing belt. \\
& Reboot power to engine controls. \\
& Restart engine. \\
\hline 2 & Run engine for 5 minutes \\
\hline 3 & Fire data capture system \\
\hline
\end{tabular}

Stop engine.

Cut power to engine controls.

Remove timing belt.

Measure and record amplifier offsets.

Refit timing belt.

Reboot power to engine controls.

Restart engine. 


\subsubsection{Modified Engine Friction Test Methodology}

As with the standard engine friction tests, the aim of the modified engine friction tests was to investigate the performance related effects of fuel administered friction modifier on measured gasoline engine friction. However, modification of the test procedure not only allowed the test series to act as a repeat for the first test series, but also served to give resolution to the time related action of the FM gasoline and allowed the evaluation of the friction data capture procedure detailed in Table 5-6. Like the standard test methodology the test began by warming the engine up to thermal equilibrium, a process which took approximately 2 hours. At this point steps 1, 2 and 3 of Table 5-6 were conducted. It was thought that the measurement of the amplifier offsets (step 4, Table 5-6) was intrusive and disturbed the operation of the engine to a greater extent than the drift it attempted to correct, for this reason it was not conducted. Friction data captures were recorded every 10 minutes when running on STD gasoline for a 30 minute period. At the 30 minute mark the fuel supply was switched to one containing FM gasoline without disruption to the engine. From this point friction data captures were taken every 2 minutes for the next 30 minutes, followed by 30 minutes where friction data was captured at ten minute intervals. The fuel supply was then switched back to STD gasoline and friction data captured every 10 minutes for a 30 minute period. It is important to note that the engine was not stopped at any point in the test, thus removing the instability associated with rebooting power to the engine controls. In addition, the exclusion of step 4 from the friction data capture procedure allows the firing of the system at 2 minute intervals, a feat not possible when adhering to the standard friction data capture procedure which requires much time to complete.

Tests were conducted at the conditions detailed in Table 5-5, although for this test series engine load was reduced to $16.5 \mathrm{Nm}$ ( $50 \%$ of full), a load which gives greater resolution to the friction data through reducing noise associated with the high load condition.

\subsection{Post Test Lubricant Analysis}

As noted previously, sump samples were collected after each test and stored in air tight containers in a refrigerated environment to preserve them prior to analysis.

\subsubsection{Fourier Transform InfraRed Microscopy (FTIR)}

The science underpinning the analysis of chemical bonds through application of the FTIR technique is described in detail in Chapter 3, section 3.5.1. However, in Chapter 3 the technique was applied with a microscope attachment, where the 
infrared beam was focused on the surface of a reflective specimen, and the reflected portion analysed for information regarding the chemical bonds. For the analysis of fluid samples such as engine lubricant the system is used in pure absorbance mode, where a small film of lubricant is sandwiched between two $\mathrm{KBr}$ discs through which the infrared beam passes. The energy absorbed through the interaction between the beam and the sample is measured by a sensitive detector capable of detailing the wave numbers at which specific absorption occurred.

Before analysing the engine sump lubricant samples, a model formulation of 2 wt\% Linoleic acid in XHVI-8.2 ${ }^{\mathrm{TM}}$ (Shell) was analysed to give a reference spectrum for a known quantity of additive in fresh sump lubricant. Subsequent analysis of sump samples was then compared with the reference spectrum giving an approximation to the level of Linoleic acid contamination in the sample. These analyses were conducted at Shell Global Solutions (UK) Analytical Laboratories by Rob Wetton.

\subsubsection{Fuel Dilution Measurement by Gas Chromatography}

Gas Chromatography (GC) analysis was conducted on sump samples at Shell Global Solutions (UK) Analytical Laboratories for fuel dilution. The process is described in detail is Chapter 3, section 2.5.1.

\subsection{Engine Performance Tests Results and Analysis}

Six identical tests were conducted to measure the effect on engine torque as a result of switching from STD to FM gasoline. As discussed in section 5.1.3 tests 2 and 5 did not produce any useful data as a result of mechanical and computational failure respectively. As a result of this, results presented in this section are those of test 1 and 3. Test 6 showed similar results to that of test 1 and 3, although with some noise in the data as a result of the poor cleanliness of the engine components as discussed in section 5.1.3. For this reason, and for concision, test 6 data has been omitted from this section.

The result taken for the first test is shown in Figure 5-5. On first inspection of the figure it is clear that changing the fuel supply from STD gasoline to FM gasoline has a measurable effect on the output torque of the engine. Although the raw trace showing engine torque variation with time shows some fluctuation in reading, the moving average trace shown in black clarifies the situation. The difference in output torque between phase 1 and phase 2 is approximately $+1.8 \%$. Interestingly, this effect is not reversed when switching back from FM gasoline to STD (Phase 2-3), although it was noted that some air was entrained into the fuel feed circuit during the 
supply switching process which made the fuel line pressure fluctuate. This problem would normally be remedied by stopping the engine and bleeding the fuel system, a procedure which was not employed in this case as it would have effectively ended the tests. Instead the engine was kept running as it was hoped the trapped air would naturally bleed from the system with time, returning the engine to stable operation. Unfortunately this was not the case as the trapped air was not expelled from the system and continued to compromise the torque measurement for the remainder of the test.

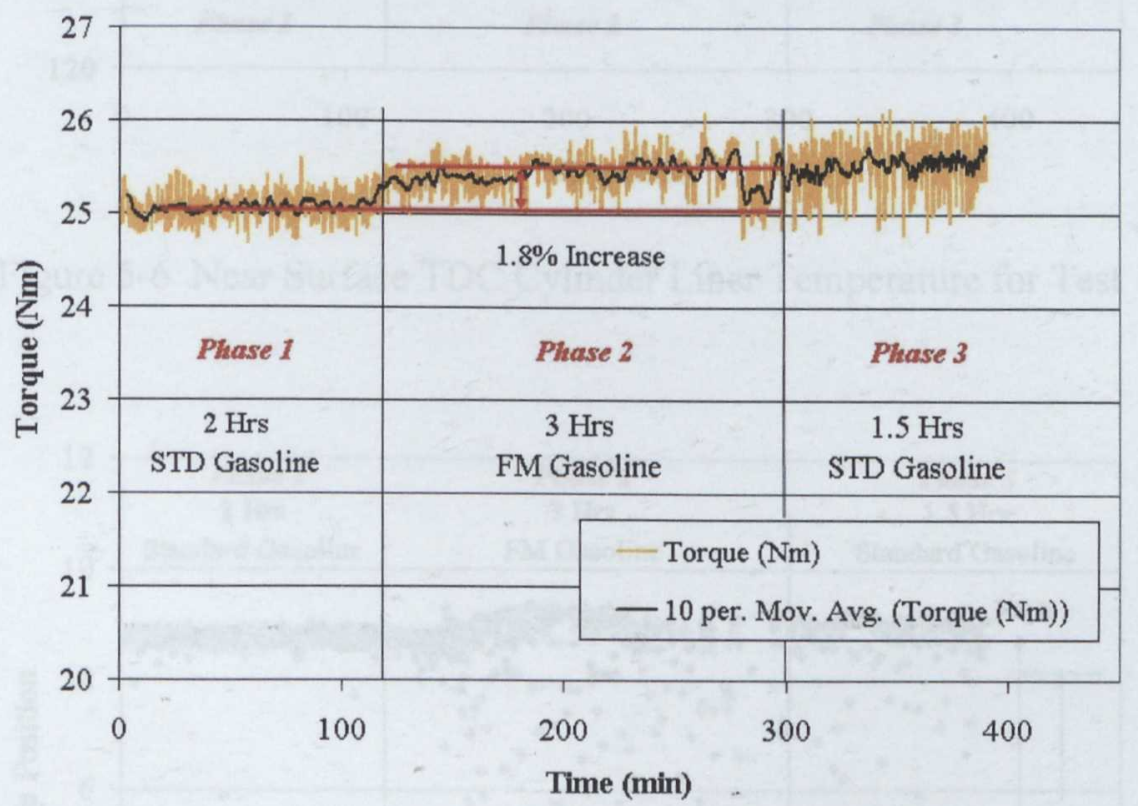

Figure 5-5 Engine Torque Measurement for Test 1

The near-surface TDC cylinder liner temperature measurement recorded for test 1 is shown in Figure 5-6. At the start of the test, in phase 1, the average near surface TDC is approximately $131^{\circ} \mathrm{C}$. This temperature appears to decrease slightly before the introduction of FM gasoline into the system (Phase 2), although remains within limits of variation previously experienced for phase 1 . The near-surface TDC temperature continues to decrease throughout phase 2 and reaches a stable value of approximately $126^{\circ} \mathrm{C}$ in phase 3 .

The exhaust gas oxygen sensor, or lambda sensor, is usually the key sensor in the engine fuel control feedback loop. In this work the sensor was used in a standalone configuration to give information regarding the stoichiometry of the combustion. The lambda sensor position response for test 1 is shown in Figure 5-7. 


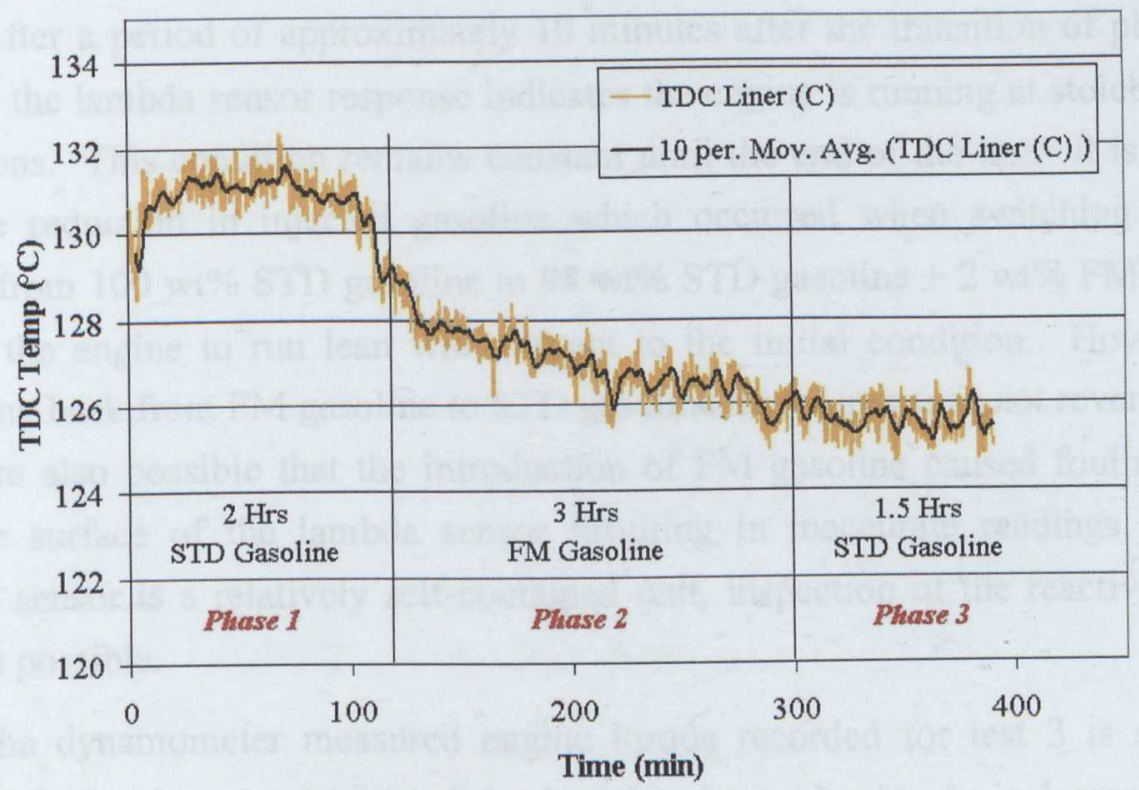

Figure 5-6 Near Surface TDC Cylinder Liner Temperature for Test 1

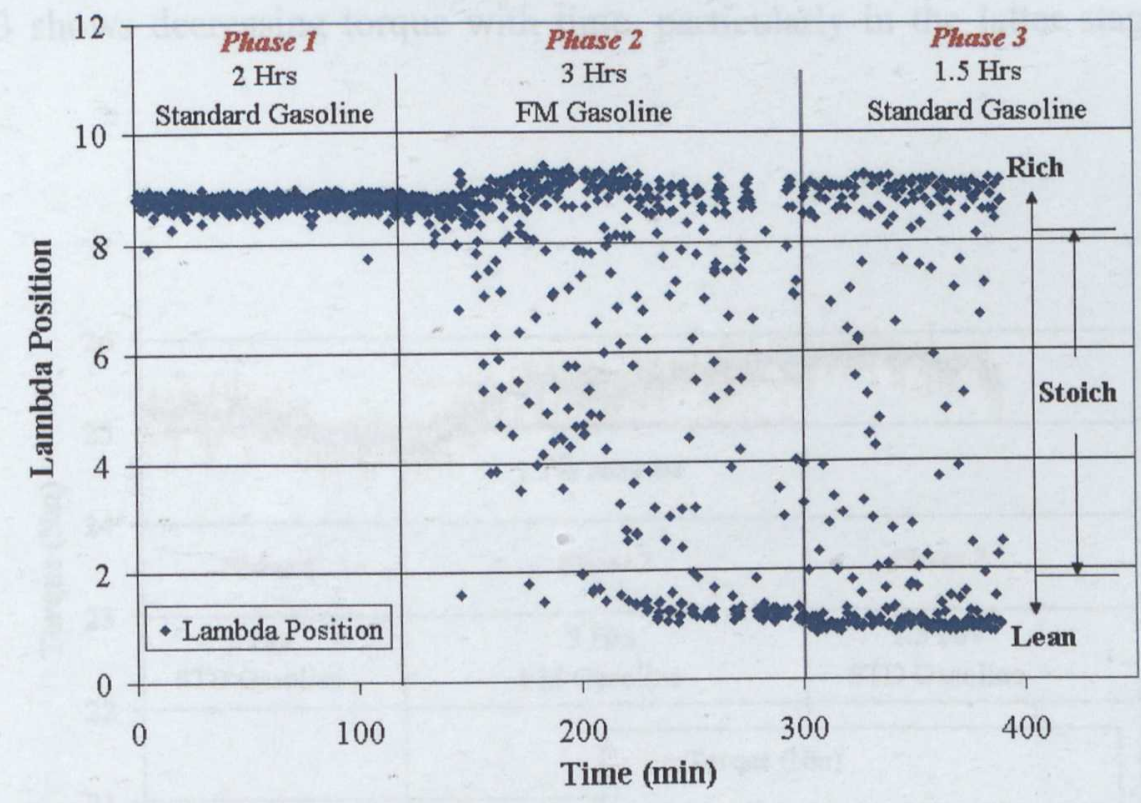

Figure 5-7 Lambda Sensor Position Response for Test 1

Interpretation of the lambda position sensor response data presented in Figure 5-7 is not as straight forward as the interpretation of the preceding graphs. The lambda position scale is non-linear with respect to variation in exhaust gas oxygen content. Typically readings in the range $2-8$ on the position scale are considered to be stoichiometric. Readings above 8 are typically indicative of a rich combustion condition, whereas those below 2 suggest the mixture condition is lean. It is clear from inspection of Figure 5-7 that the test begins with the engine running slightly 
rich. After a period of approximately 10 minutes after the transition of phase 1 to phase 2 the lambda sensor response indicates the engine is running at stoichiometric conditions. This condition remains constant until the end of the test. It is possible that the reduction in injected gasoline which occurred when switching the fuel supply from $100 \mathrm{wt} \%$ STD gasoline to $98 \mathrm{wt} \%$ STD gasoline $+2 \mathrm{wt} \%$ FM gasoline caused the engine to run lean with respect to the initial condition. However, on switching back from FM gasoline to STD gasoline this response is not reversed. It is therefore also possible that the introduction of FM gasoline caused fouling of the reactive surface of the lambda sensor resulting in inaccurate readings. As the lambda sensor is a relatively self-contained unit, inspection of the reactive surface was not possible.

The dynamometer measured engine torque recorded for test 3 is shown in Figure 5-8. In phase 1 of the test it is clear that the engine torque is decreasing with time until approximately 10 minutes into phase 2 where FM gasoline was introduced to the engine. From this point engine torque continues to rise throughout the 3 hour period with an average increase of $1.7 \%$ compared with the baseline set in phase 1 . Phase 3 shows decreasing torque with time, particularly in the latter stages of the test.

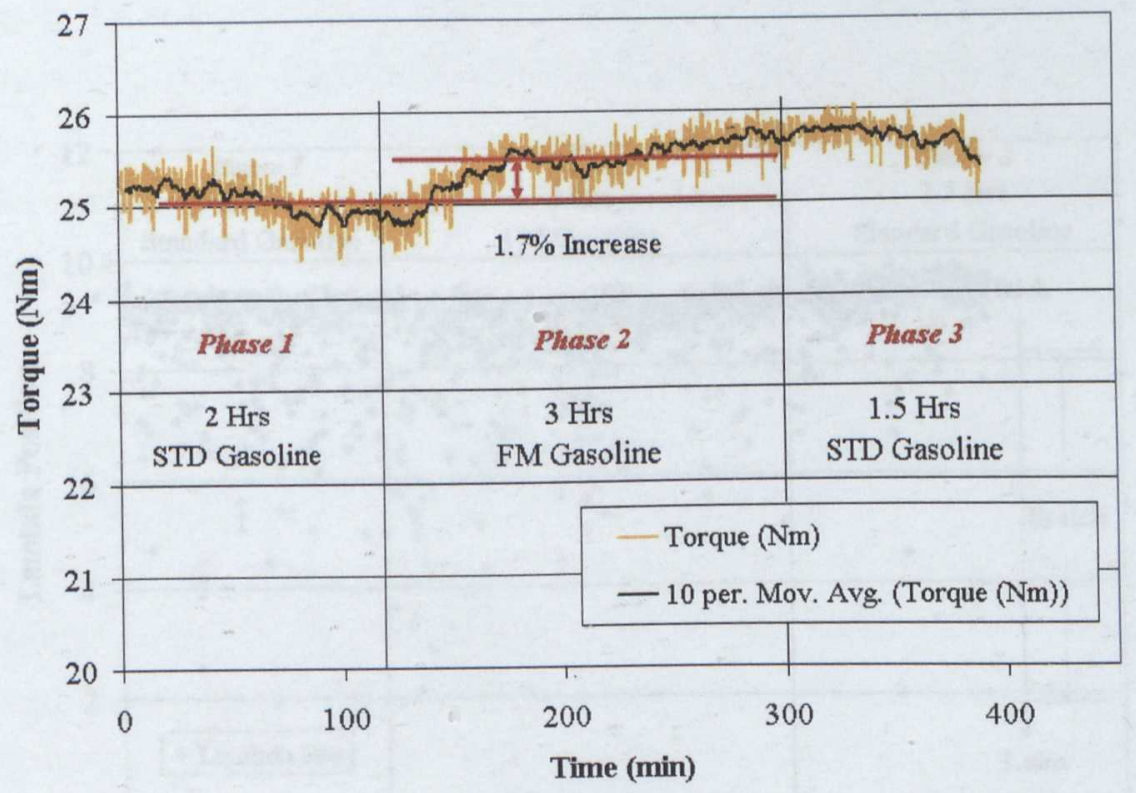

Figure 5-8 Engine Torque Measurement for Test 3

The near-surface TDC cylinder liner temperature recorded for test 3 is shown in Figure 5-9. Phase 1 shows a decreasing temperature trend with time, with temperature falling approximately $1^{\circ} \mathrm{C}$ per hour for the duration of the two hour period. Phase 2 initially shows a similar rate of decrease in temperature as that 
shown in phase 1 , although after approximately 30 minutes the temperature drops rapidly at a rate of approximately $2.5^{\circ} \mathrm{C}$ per hour settling at a value around $122^{\circ} \mathrm{C}$ for the remaining duration of the phase. Immediately after transition to phase 3 the near-surface TDC temperature rises rapidly, settling at a value around $124^{\circ} \mathrm{C}$.

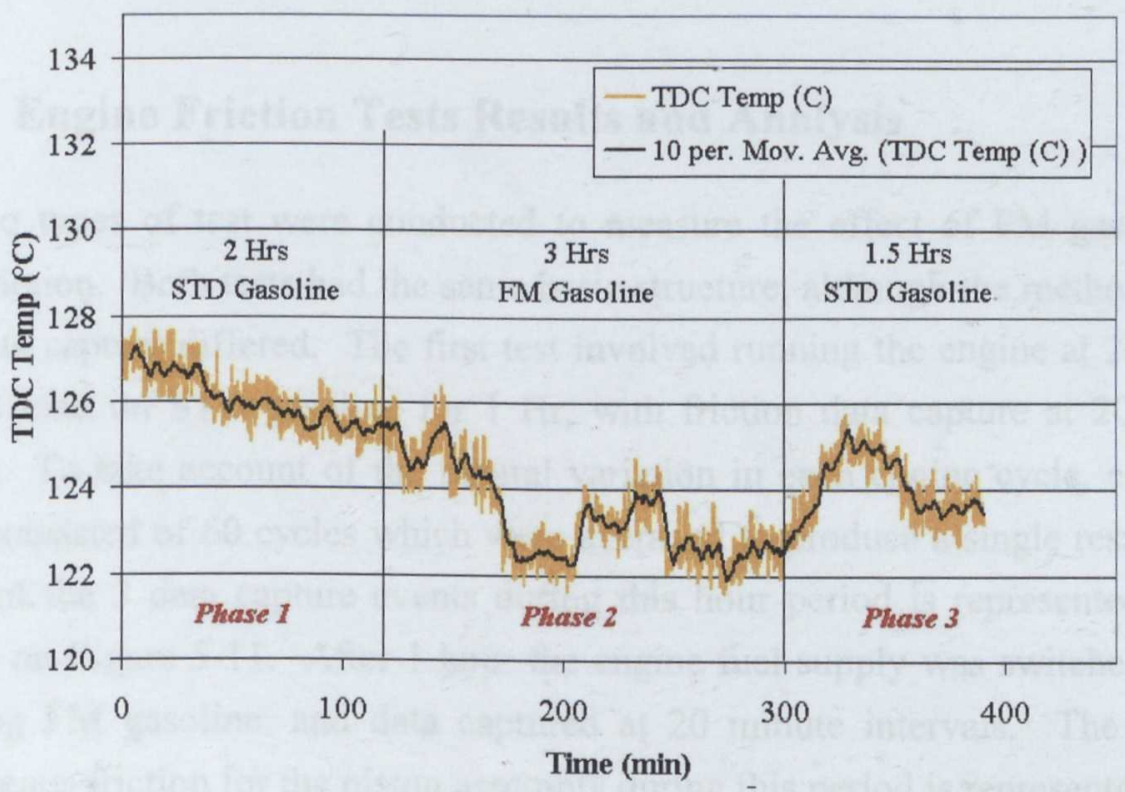

Figure 5-9 Near-surface TDC Cylinder Liner Temperature for Test 3

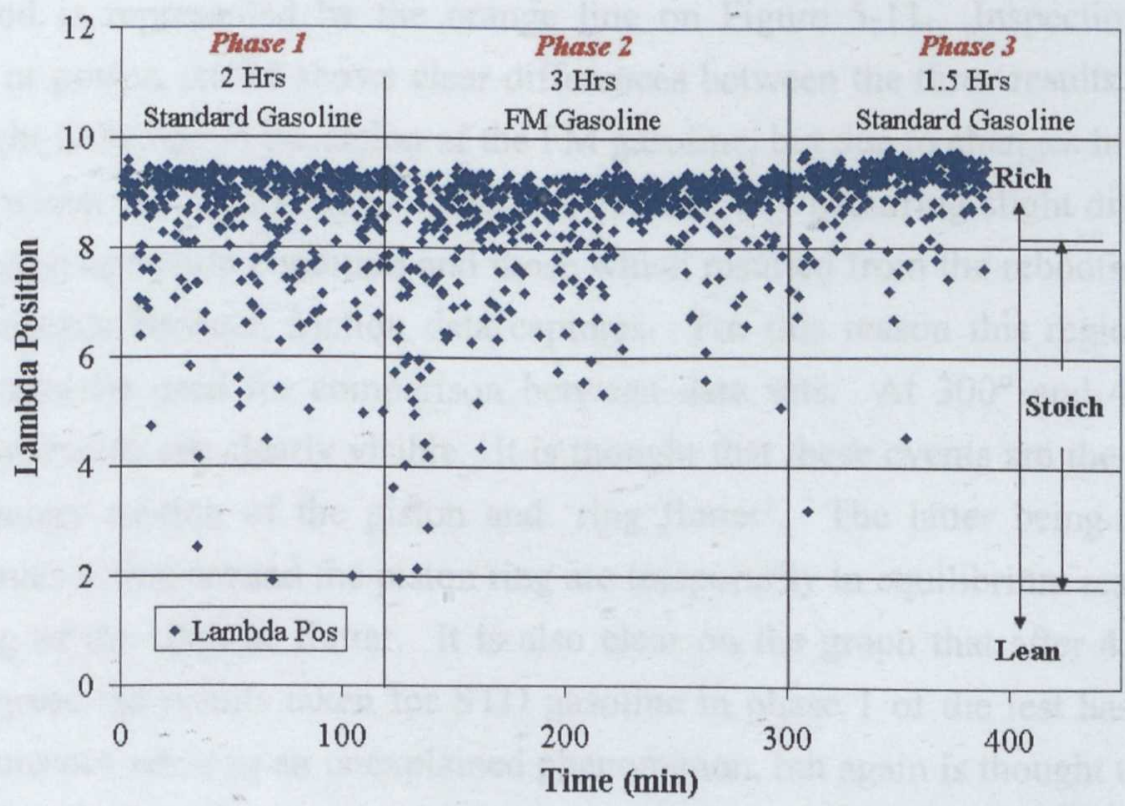

Figure 5-10 Lambda Sensor Position Response for Test 3

The lambda sensor response for test 3 is shown in Figure 5-10. Before each test the lambda sensor was checked by varying the air / fuel ratio of the engine and 
ensuring the response was both correct and immediate. In phase 1 of the test the sensor response shows the engine is running at a slightly rich condition, a condition which remains unchanged throughout phase 2 where FM gasoline is introduced to the engine. During phase 3 of the test the response of the lambda sensor suggests the engine was running slightly richer than the preceding phases, although not by any significant amount.

\subsection{Engine Friction Tests Results and Analysis}

Two types of test were conducted to measure the effect of FM gasoline on engine friction. Both tests had the same basic structure, although the methodologies of the data capture differed. The first test involved running the engine at $2000 \mathrm{rpm}$ and $60 \%$ load on STD gasoline for $1 \mathrm{Hr}$, with friction data capture at 20 minute intervals. To take account of the natural variation in each engine cycle, each data capture consisted of 60 cycles which were averaged to produce a single result. The average of the 3 data capture events during this hour period is represented by the blue line on Figure 5-11. After 1 hour the engine fuel supply was switched to one containing FM gasoline, and data captured at 20 minute intervals. The average instantaneous friction for the piston assembly during this period is represented by the pink line on Figure 5-11. Finally, the fuel supply was switched back to one containing STD gasoline and run for $1 \mathrm{Hr}$, again with friction data capture at 20 minute intervals. The average instantaneous friction for the piston assembly during this period is represented by the orange line on Figure 5-11. Inspection of the ignition, or power, stroke shows clear differences between the three results. This is not thought to be due to the action of the FM gasoline, but due to changes in cylinder pressure which occurred both as a result of the naturally occurring slight differences in the engine operation condition and those which resulted from the rebooting of the engine controls between friction data captures. For this reason this region of the graph cannot be used for comparison between data sets. At $300^{\circ}$ and $450^{\circ}$ two significant events are clearly visible. It is thought that these events are the result of the secondary motion of the piston and 'ring flutter'. The latter being an event where forces acting around the piston ring are temporarily in equilibrium resulting in the lifting of the ring, or flutter. It is also clear on the graph that after 450 crank angle degrees the results taken for STD gasoline in phase 1 of the test has drifted. This occurrence remains an unexplained phenomenon, but again is thought to be due to changes to the measurement equipment as a result of rebooting the engine controls. Despite the anomalies associated with this test, there appears to be significant differences between the results which allow differentiation between the 
piston assembly friction condition when running on STD gasoline and FM gasoline. These differences are most easily explained through consideration of Figure 5-12.

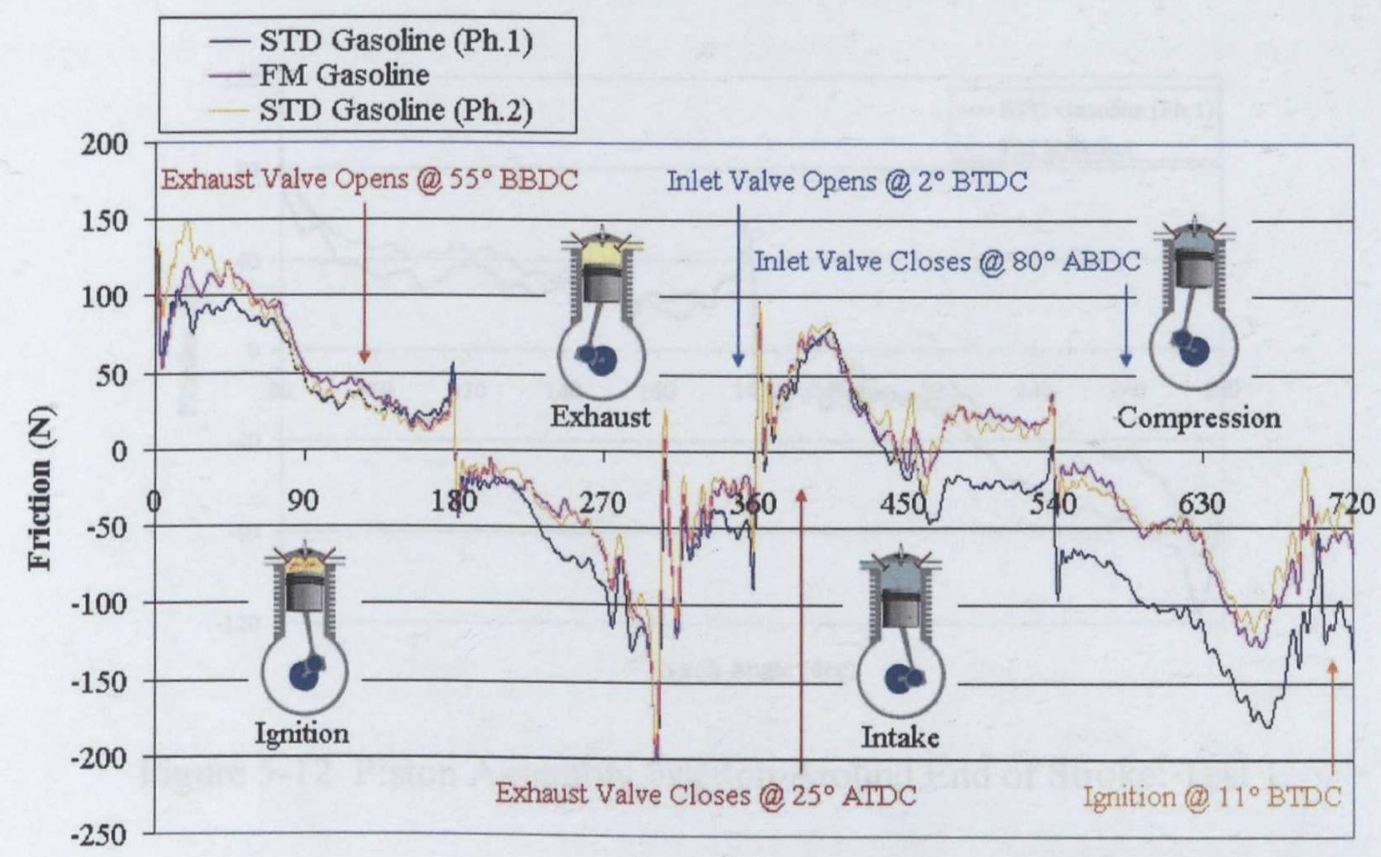

Crank Angle (deg)

Figure 5-11 Piston Assembly Friction Measurement and Key Engine Events: Test 1

The friction force for the piston assembly in the region after combustion, from $80^{\circ}$ to $280^{\circ}$ is shown on Figure 5-12. This figure magnifies the differences between the average instantaneous piston assembly friction when running on STD and FM gasoline. The assembly is thought to enter the mixed lubrication regime at approximately $150^{\circ}$. At this point it is clear that the measured friction force when running on FM gasoline is lower than when running on STD gasoline. This trend continues into the boundary lubrication regime, the area surrounding the piston reversal at $180^{\circ}$. Once the piston motion is reversed, it is clear that the friction force measured for FM gasoline remains considerably less than that for STD gasoline until $280^{\circ}$ where lubrication is thought to be dominated by hydrodynamics. It is interesting to note that the FM gasoline appears to reduce friction on the upstroke more significantly than on the down-stroke, particularly in the area thought to be associated with the mixed lubrication regime. Figure 5-13 shows the impact of the addition of FM gasoline by considering the friction reduction with respect to piston velocity, presenting piston friction power loss against crank angle. The result clarifies the importance of reducing friction in the mixed lubrication regime, where piston velocities are relatively high. The region around $250^{\circ}$ magnifies the energy saving achieved through application of FM gasoline. It is important to remind the 
reader that the large differences between FM gasoline and STD gasoline from $450^{\circ}$ onwards are as a result of drift in the measurement, and not the effect of FM gasoline.

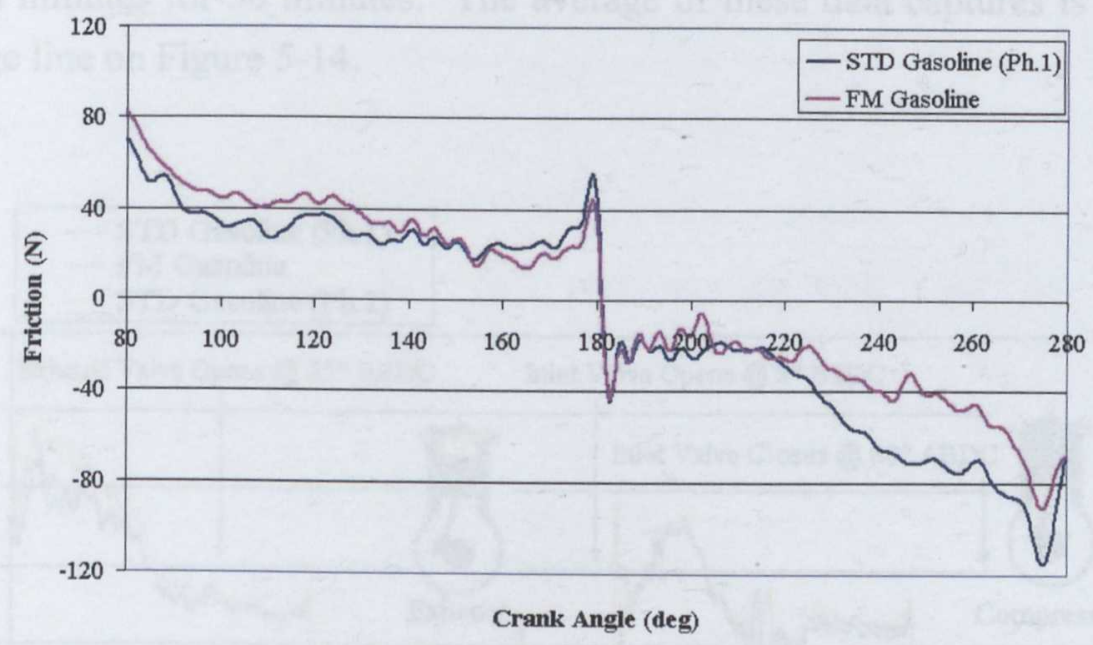

Figure 5-12 Piston Assembly Friction Around End of Stroke: Test 1

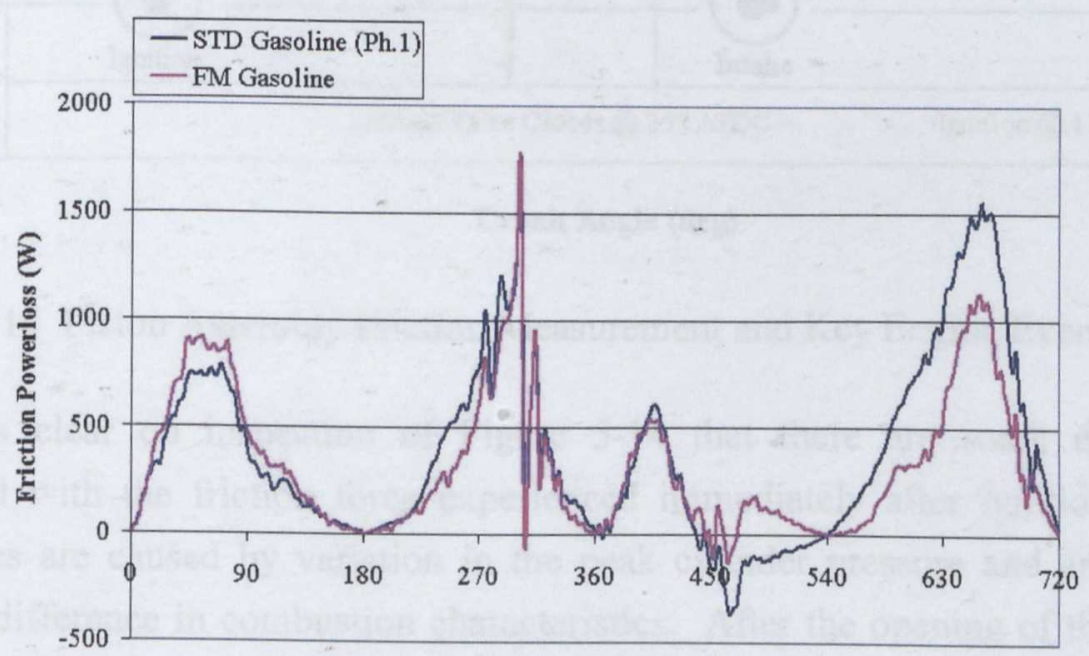

Crank Angle (deg)

Figure 5-13 Piston Assembly Friction Power Loss: Test 1

The second set of tests followed the same basic structure as the first, except the engine was not stopped at any point throughout the test duration. Again a 60 cycle average was conducted at every data capture to take account for the natural variation in engine operation with each cycle. The blue line on Figure 5-14 represents the average of the 3 data capture events which took place every ten minutes for the first 30 minutes. When running on FM gasoline, data capture occurred every 2 minutes for the first 30 minutes, and every 10 minutes for the following 30 minutes. The 
pink line on the graph represents an average of the data captured in this time, excluding the first ten minutes which is the estimated time period it takes for FM gasoline to reach the fuel injector once the supply switch has occurred. In Phase 3 of the test the fuel supply was switched back to STD gasoline and friction data captured every ten minutes for 30 minutes. The average of these data captures is shown by the orange line on Figure 5-14.

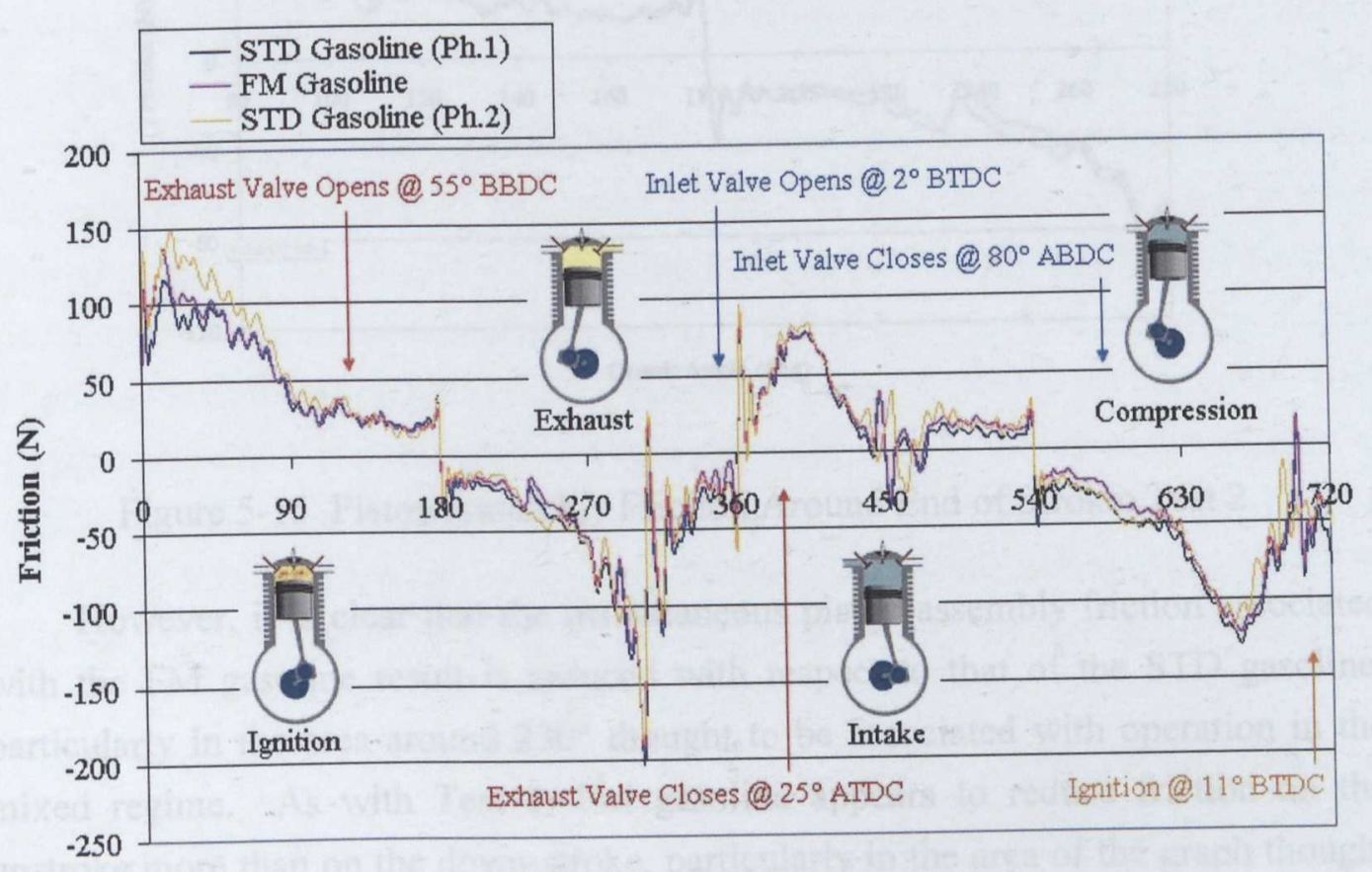

Crank Angle (deg)

Figure 5-14 Piston Assembly Friction Measurement and Key Engine Events: Test 2

It is clear on inspection of Figure 5-14 that there are some differences associated with the friction force experienced immediately after ignition. These differences are caused by variation in the peak cylinder pressure and are a direct result of difference in combustion characteristics. After the opening of the exhaust valve at $125^{\circ}$ the engine is effectively doing pumping work for the remaining $595^{\circ}$ of the cycle. It is after this point that the effects of the FM gasoline can be seen with respect to running on STD gasoline. Similarly to the first set of tests conducted with the standard test methodology, there are events at $300^{\circ}$ and $450^{\circ}$ thought to be due to the secondary motion of the piston and ring flutter respectively. Again the differences in instantaneous piston friction are best quantified by considering a small portion of the cycle in detail, as is depicted in Figure 5-15. On first inspection it is evident that the difference in measured friction between STD and FM gasoline is much less evident than in Figure 5-14 associated with Test 1. It is possible this is the result of the decreased load the engine was run at for these tests, or the build up 
of varnish on the components shown to occur with engine hours as detailed in section 5.1.3.

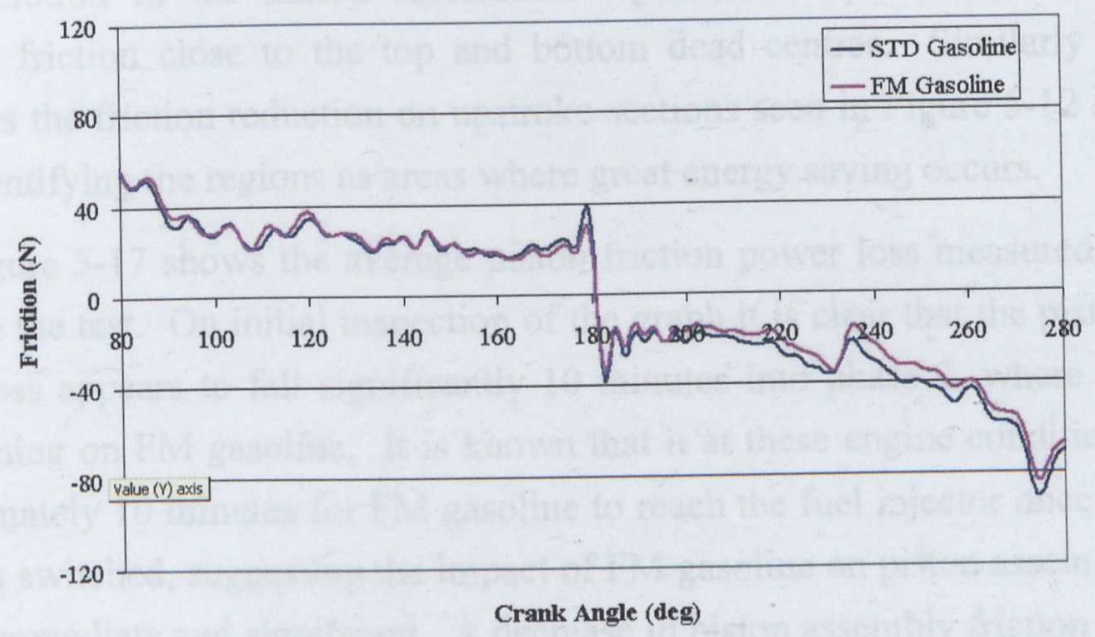

Figure 5-15 Piston Assembly Friction Around End of Stroke: Test 2

However, it is clear that the instantaneous piston assembly friction associated with the FM gasoline result is reduced with respect to that of the STD gasoline, particularly in the area around $230^{\circ}$ thought to be associated with operation in the mixed regime. As with Test $1, \mathrm{FM}$ gasoline appears to reduce friction on the upstroke more than on the down-stroke, particularly in the area of the graph thought to be associated with operation in the mixed regime $\left(\sim 230^{\circ}\right)$.

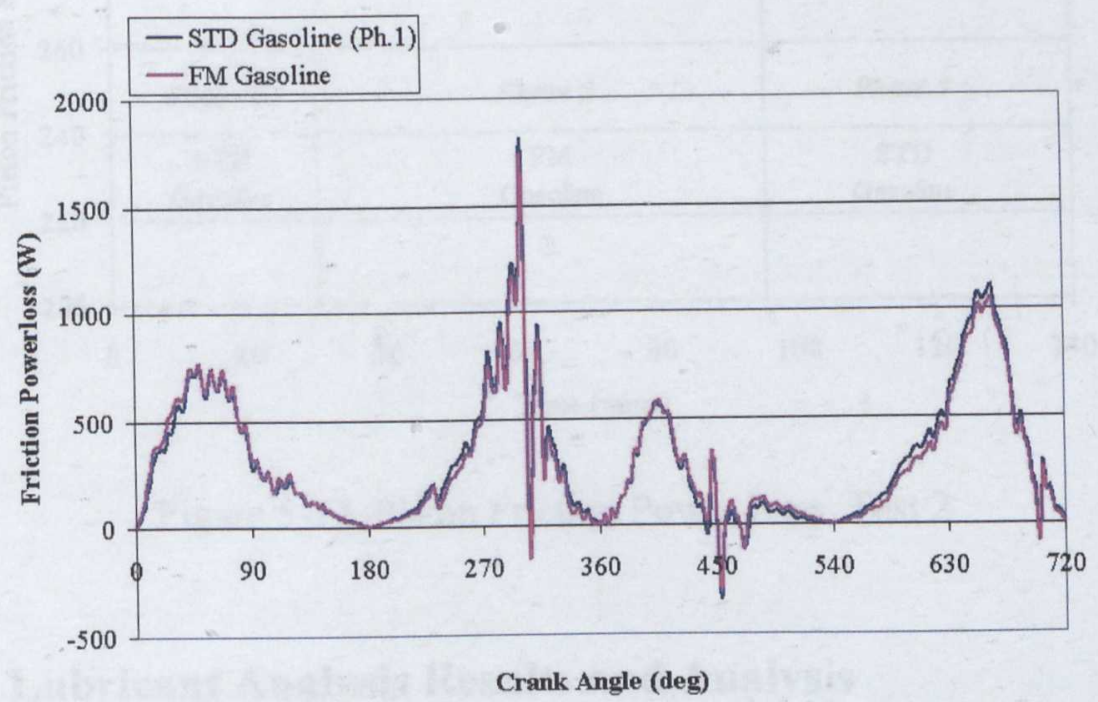

Figure 5-16 Piston Assembly Friction Power Loss: Test 2 
The piston assembly friction power loss for both STD and FM gasoline are shown in Figure 5-16. From this graph it is clear that FM gasoline reduces piston assembly friction power loss most significantly in the areas thought to be associated with operation in the mixed lubrication regime, and quantifies the impact of reducing friction close to the top and bottom dead centres. Similarly the figure quantifies the friction reduction on upstroke sections seen in Figure 5-12 and Figure $5-15$, identifying the regions as areas where great energy saving occurs.

Figure 5-17 shows the average piston friction power loss measured at various stages in the test. On initial inspection of the graph it is clear that the piston friction power loss appears to fall significantly 10 minutes into phase 2 , where the engine was running on FM gasoline. It is known that it at these engine conditions it takes approximately 10 minutes for FM gasoline to reach the fuel injector once the supply has been switched, suggesting the impact of FM gasoline on piston assembly friction is both immediate and significant. A decrease in piston assembly friction power loss of approximately $3.5 \%$ was recorded when running on FM gasoline with respect to running on STD gasoline. It is clear on the graph that the piston assembly friction power loss does not return to baseline levels once the fuel supply is switched back to STD gasoline, the system continues to operate with reduced friction during phase 3.

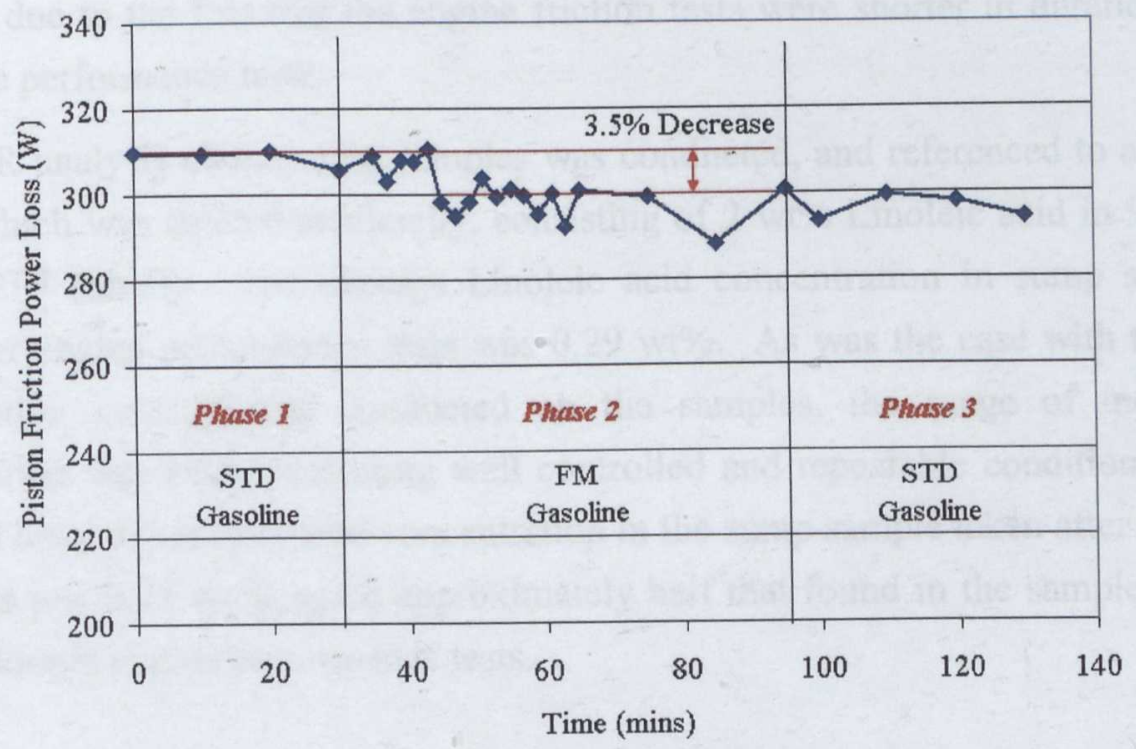

Figure 5-17 Piston Friction Power Loss: Test 2

\subsection{Lubricant Analysis Results and Analysis}

Samples were tested for both fuel and Linoleic acid concentration using gas chromatography and FTIR techniques respectively. The results of analyses are 
presented in Table 5-7. The sump sample associated with Friction test 2 was not analysed due to a spill which occurred during transportation to the analytical laboratory.

\begin{tabular}{ccc}
\hline Test & $\begin{array}{c}\text { Fuel } \\
\text { Concentration (wt\%) }\end{array}$ & $\begin{array}{c}\text { Linoleic Acid } \\
\text { Concentration (wt\%) }\end{array}$ \\
\hline Performance Test 1 & 1.53 & 0.29 \\
\hline Performance Test 3 & 1.56 & 0.28 \\
\hline Performance Test 6 & 1.31 & 0.30 \\
\hline Friction Test 1 & 0.75 & 0.13 \\
\hline
\end{tabular}

Table 5-7 Sump Lubricant Sample Fuel and Linoleic Acid Concentration

The average fuel dilution measured in the sump lubricant after an engine performance test was $1.46 \mathrm{wt} \%$. The similarities in the measured fuel dilutions are an indication that the engine conditions were well standardised, and that tests were uniform. For the friction test, the level of fuel dilution was approximately half of that typically found in sump lubricant taken after engine performance tests. This is primarily due to the fact that the engine friction tests were shorter in duration than the engine performance tests.

FTIR analysis of the sump samples was conducted, and referenced to a model sample which was created artificially, consisting of $2 \mathrm{wt} \%$ Linoleic acid in $98 \mathrm{wt} \%$ XHVI-8.2 ${ }^{\mathrm{TM}}$ (Shell). The average Linoleic acid concentration in sump samples taken after engine performance tests was $0.29 \mathrm{wt} \%$. As was the case with the fuel concentration measurement conducted on the samples, the range of measured concentration was small indicating well controlled and repeatable conditions. The estimated level of Linoleic acid concentration in the sump sample taken after friction test 1 was just $0.13 \mathrm{wt} \%$, again approximately half that found in the samples taken after the longer engine performance tests.

\subsection{Discussion}

\subsubsection{Engine Performance Tests Discussion}

Engine brake torque was shown to significantly increase through the application of gasoline containing friction modifier. It is thought that the friction modifier, which enters the combustion chamber with the gasoline, mixes with the lubricant film on both the cylinder liner and piston assembly where it is protected 
from the combustion event. The additive film then acts to reduce the coefficient of friction in the contact allowing more of the energy supplied to the system by the fuel to be converted into useful work. It was shown in previous chapters that the tenacity of the fatty acid molecule is relatively weak, suggesting additive films are probably readily removed from the surface by the scraping action of the piston rings. However, as additive is administered to the surfaces with every opening of the fuel injector supplies of additive are constantly being replenished, resulting in effective continual modification of the contact condition. It is hypothesised that the system may be operating under what is known as 'Forced Equilibrium' conditions. Although additive molecules are removed from the surface by the scraping action of the piston rings, fresh supplies of additive are always available to form where metal is exposed thus maintaining an effective surface film.

Typical results showed an approximate $2 \%$ increase in brake torque when running on FM gasoline with respect to STD gasoline. However, this effect is not reversed when switching from FM gasoline to STD gasoline, although Figure 5-8 shows some indication that there is a reduction in torque with time. It is possible that had the test run for longer engine torque may have returned to the benchline value established at the start of the test. There are two main hypotheses which explain this phenomenon. The first recognises that the tenacity of the films were shown to be weak in the arduous conditions simulated by bench-top tribometer tests, and conjects that conditions in the engine might be less difficult resulting in the increased tenacity of the additive films. The second is based on the knowledge gained as a result of the lubricant sampling tests which were presented earlier in this thesis where additive was shown to accumulate in both the top ring groove and cylinder liner oil film in considerable quantities. This lead to the hypothesis stating that although FM gasoline was no longer being applied to the system, a significant excess of accumulated active additive in the lubricant continued to maintain an effective friction modifier film for a period of time. The third hypothesis is again based on findings from the lubricant sampling tests which identified two main transport mechanisms of gasoline to the sump lubricant; liquid and vapour phase transport. This hypothesis acknowledges that although the bulk of the gasoline administered friction modifier was probably burnt and exited the engine via the exhaust, a significant quantity of additive is likely to be transported to the sump lubricant by the two mechanisms discussed above. Once in the engine lubricant the FM additive is available to reduce friction in a manner more typical of a fully formulated engine oil containing an FM additive. However, results presented in Table 5-7 show a typical $0.3 \mathrm{wt} \%$ concentration of Linoleic acid in the sump lubricant, a quantity not thought to be large enough to have a significant effect on engine friction. 
Two other interesting effects were recorded during the engine performance tests, a reduction in near-surface cylinder liner temperature and variation in exhaust gas oxygen concentration. When running on FM gasoline the near-surface cylinder liner temperature was typically $2{ }^{\circ} \mathrm{C}$ less than when run on STD gasoline. This result is particularly interesting when given context by the exhaust gas oxygen sensor reading for the same test. Typically the engine was thought to run at a leaner mixture condition when operating on FM gasoline, a result of the fuel supply containing $2 \mathrm{wt} \%$ less gasoline. It is widely understood that running an engine at lean conditions will increase exhaust gas temperature, in turn raising the temperatures of components associated with the combustion chamber. It is possible that the reduction in near-surface cylinder liner temperature measured when FM gasoline was applied to the system is a direct result of reducing engine friction. However, it is the view of the author that the increase in component temperature caused by leaning the combustion mixture would far outweigh any temperature reduction caused by reduced friction in the contact. However, it is equally possible that at the start of the test the engine was running rich enough to absorb the leaning of the mixture caused through the switching of the fuel supply to FM gasoline, remaining predominantly rich and allowing the reduction in near-surface temperature to be a significant measurable quantity.

\subsubsection{Engine Friction Tests Discussion}

Following a similar structure to the engine performance tests, the engine was equipped with apparatus allowing the measurement of piston assembly friction under firing conditions. The first set of engine tests were conducted using a standardised methodology which involved rebooting the power to the test cell after every data capture. Although this procedure is thought to have created some variation in the operation of the engine, the effects of running the engine on FM gasoline were still clearly measurable. Results taken from the test showed reduced friction operation of the piston assembly when operating in both the boundary and mixed lubrication regimes. This is consistent with bench-top tribometer test results presented in earlier chapters suggest Linoleic acid to be effective in both lubrication regimes. The piston assembly friction measurement highlights an interesting effect where the friction measured when operating in the mixed lubrication regime during the upstroke was reduced to a greater extent than on the equivalent down-stroke. The hypothetical explanation for this is that the napier scraper faced profile of the second compression ring is well separated from the liner surface by the additive film on the upstroke due to its wedge shape, but less effectively separated on the down-stroke where its design ensures an aggressive scraping action. Essentially the additive film is capable of reducing the contact friction between cylinder liner and both first and second 
piston rings on the upstroke, but is less effective at reducing the second compression ring friction on the down-stroke, thus friction reduction in this area is mainly associated with the action of the top compression ring.

Calculation of piston assembly friction power loss reveals the areas of the engine cycle where the FM additive enhances energy savings most. Although with FM gasoline friction is shown to be lowered around the piston reversal points, or end-stroke, the low velocity in these regions means energy saving is low. In the mixed regime, where piston speeds are relatively high, the greatest energy saving is achieved.

Analysis of the sump lubricant sample taken on completion of the test showed it to contain $0.75 \mathrm{wt} \%$ gasoline and $0.13 \mathrm{wt} \%$ Linoleic acid. This is approximately half the concentration measured in samples taken after the engine performance tests, and is thought to be a result of the difference in the duration of the tests. During the engine performance tests the FM additive was injected into the engine for 3 hours, whereas for the engine friction tests it was only injected for 1 hour. This level of Linoleic acid contamination in the sump lubricant is not thought to be high enough to significantly affect engine friction.

The second set of piston assembly friction measurement tests were conducted at similar conditions to the first, although at $50 \%$ load instead of $60 \%$. To eliminate engine operation variation as a result of cutting power to the test cells, a procedure conducted to measure the amplifier drifts, the test methodology was changed allowing the engine to run undisturbed from the start of the test to the finish. This methodology allowed the determination of the speed of reaction of the FM additive as a result of the increased test resolution. Data captured during these tests highlights the effectiveness of the method as many of the effects resulting from variation in engine condition as a result of the amplifier drift measurement methodology were eliminated. As with the first test, results highlight the effectiveness of Linoleic acid at reducing piston assembly friction in both the boundary and mixed lubrication regimes, as well as the enhanced ability of the additive to reduce friction on the piston upstrokes. However, the effects of the additive appear to be reduced when compared with those measured in test 1 . There are two hypotheses which can explain this phenomenon. The first suggests the reduced engine load condition used in test 2 results in less arduous asperity contact, thus the need for FM additive is reduced. The second hypothesises that the engine surfaces were coated in a varnish formed due to the lack of detergent in either the lubricant or fuel. The varnish reduces the number of sights onto which the additive can form, thus reducing the effective impact of the friction modifier. 
Consideration of friction reduction with respect to piston velocity, or piston assembly friction power loss, shows us that the FM gasoline saves a great deal of energy through reducing friction where operation is in the mixed lubrication regime, although it is clear on inspection of Figure 5-16 that energy saving occurs through most of the engine cycle when FM additive is used.

The modified test methodology allowed data capture to occur at more frequent intervals, permitting the determination of the speed of reaction of the FM gasoline. Figure 5-17 shows the average piston assembly friction power loss for each data capture conducted throughout the test. From inspection of this figure it is immediately apparent that friction power loss is reduced approximately 10 minutes after switching the fuel supply from STD gasoline to FM gasoline, showing the impact of FM gasoline on friction power loss to be both immediate and significant. As discussed earlier, it is estimated that it took 10 minutes for the FM gasoline to reach the fuel injector once the fuel supply had been switched from STD gasoline. There was a $3.5 \%$ decrease in average friction power loss through the application of FM gasoline with respect to STD gasoline, an effect which did not reverse once switchback to STD gasoline was initiated. The possible reasons why the friction power loss did not return to the benchline value measured at the start of the test were discussed earlier.

\subsection{Summary}

In this chapter a series of engine tests were described where the effects of gasoline doped with friction modifier additive were tested. Preliminary engine tests were conducted to 'scout' the effects of gasoline containing friction modifier additive. These tests measured the effect on dynamometer engine brake torque as a result of switching from standard gasoline to gasoline containing friction modifier. A second series of tests were conducted which used the Instantaneous Mean Effective Pressure method to directly measure changes in piston assembly friction as a result of switching from standard gasoline to gasoline containing friction modifier. On consideration of all results, analysis and discussion presented in this chapter, the following summary can be made.

- Engine output torque was shown to increase approximately $2 \%$ through application of gasoline containing Linoleic acid friction modifier at 2 wt $\%$.

- Friction modifier additive is thought to accumulate in the lubricant film on both piston assembly and cylinder liner and continues to effect the 
piston assembly lubrication after the supply of gasoline containing friction modifier has been removed.

- The additive system is thought to operate in forced equilibrium conditions, where additive molecules removed from the surface are immediately replaced by surrounding molecules maintaining an effective surface film.

- Near-surface cylinder liner temperature was shown to reduce approximately $2^{\circ} \mathrm{C}$ with the application of gasoline containing friction modifier, although varying engine condition makes it impossible to suggest this effect was due solely to friction reduction in the contact between piston assembly and cylinder liner.

- When run on gasoline containing friction modifier, the engine sump lubricant was shown to contain measurable amounts of friction modifier. Friction modifier additive is thought to be transported to the engine sump via both vapour phase and liquid transport mechanisms.

- Gasoline containing friction modifier was shown to considerably reduce piston assembly friction through modification of the lubrication conditions in both the boundary and mixed lubrication regimes.

- Appreciable energy savings were shown to result through application of gasoline containing friction modifier, principally as a result of the reduced friction in the mixed lubrication regime.

- The effect of gasoline containing friction modifier was shown to be most pronounced on the piston upstroke, where the action of the additive is thought to influence the lubrication condition of the second compression ring in addition to that of the top compression ring.

- Gasoline containing friction modifier was shown to have an immediate and significant effect on reducing engine friction after entering the engine.

- The findings of the engine tests presented in this chapter are consistent with bench-top tribometer tests and show fatty acid friction modifier to be effective at reducing the friction of the piston assembly. 


\section{Discussion}

This thesis is presented in a rigid structure where each chapter represents a stand-alone section of work. It is the purpose of this chapter to discuss the research project in its entirety, drawing together the findings from all chapters and interpreting the conclusions with respect to each other. Only in considering all results presented in this thesis as a linked data set can an evaluation of the impact in totality of friction modified gasoline be made.

In this chapter the key findings of the research project are discussed, where possible with reference to the research of others as detailed in Chapter 1, and with reference to the objectives outlined in the same chapter. The interpretation of results which are seminal in this field of research is also conducted, with a view to building up a more knowledgeable picture of the way in which environmentally friendly friction modifier additives can work in automotive applications. Finally the impact of this type of research is discussed with respect to the motorist, society and the world.

\subsection{Key Project Findings}

It was stated in Chapter 1 that the presence of fuel in the piston assembly / cylinder liner lubricant film is a widely observed occurrence, although previous research gives little quantification of the phenomenon. In Chapter 2 a series of incylinder lubricant sampling tests were presented which scoped the possibility of administering friction modifier directly to the piston assembly and cylinder liner walls, whilst giving information detailing fuel contamination in the lubricant. Tests were conducted to extract lubricant from the top ring groove of the piston during firing engine conditions. Using standard gasoline and running at varying engine conditions the typical level of fuel dilution found in the lubricant samples extracted from the top ring groove ranged from $5-11 \mathrm{wt} \%$. This work contrasts with that of Gamble (2002a) where a similar set of tests were conducted on the same engine and show fuel dilution to range between $10-80$ vol\%. However, work conducted by Kim et al (2003) explains that fuel contamination in the cylinder liner oil film is heavily influenced by valve timing, injector timing and engine speed, factors which may explain experimental differences. Tests conducted in this research project did not investigate the impact of varying valve or injector timing, but did investigate the impact of varying engine speed on fuel contamination in the piston assembly lubricant, showing it to have some effect. 
The final boiling point of the gasoline was shown to be a significant factor influencing the level of fuel contamination in the piston assembly lubricant, as was also found by Frottier et al (1996). Research conducted in this project showed 33 $w t \%$ fuel in the top ring zone sample when the engine was run on high boiling point fuel.

The tests also served to investigate the possibility of administering friction modifier to the piston assembly lubricant using gasoline. In the top ring groove, additive was shown to accumulate in proportions up to 77 times greater than the initial treatment level of the fuel. Lubricant extracted from the top of the cylinder liner was shown to contain large amounts of additive; up to a level 5 times greater than the initial treatment level of the fuel. The amount of additive administered to the cylinder liner oil film was shown to decrease towards the bottom of the cylinder liner as expected, although it was still found in significant proportions.

Tests conducted from cold start engine conditions looked at the influence of the final boiling point of the gasoline on fuel dilution in the cylinder liner and sump lubricant (Section 2.7.1). Results of the experiments showed a significant increase in the level of sump fuel dilution when running on high boiling point gasoline with respect to tests conducted on standard gasoline. The level of fuel found in the sump after running on high boiling point fuel for four hours appeared to stabilise at around $1.7 \mathrm{wt} \%$, whereas the level of fuel found in the test run on standard gasoline appeared to stabilise at around $1.1 \mathrm{wt} \%$. It is interesting to compare these findings with those of other researchers who have investigated sump fuel dilution. As discussed in Chapter 1, Thompson (1997) developed a numerical model to simulate the transport and accumulation of fuel in the lubricant sump, the conditions of which are shown in Table 6-1 and are presented alongside the conditions of similar tests conducted by Murakami and Aihara (1991) and the author of this thesis. Interestingly, Thompson's numerical approximation to sump fuel dilution predicted an equilibrium concentration of around $1.9 \mathrm{wt} \%$, whilst Murakami and Aihara measured 1.7 wt\% fuel dilution in the sump under equilibrium conditions. The author of this thesis suggests that the accumulation of fuel in engine sump lubricant is governed by much more complex mechanisms than those which can be defined through use of the parameters described in Table 6-1, although evidence suggests that models based on the parameters can approximate to experimental findings. Research reported in Chapter 2 of this thesis concludes that the transport of fuel to the engine sump is thought to take place by one of two mechanisms; 1) fuel passes the piston rings in the blow-by gasses in a vapour phase, 2) fuel adsorbs into the cylinder liner lubricant, which is returned to the sump in a liquid phase through the dynamic action of the piston ring / cylinder liner interface. 
The knowledge that fuel passes the piston assembly and enters the engine sump lubricant combined with lateral and innovative thinking sparks the hypothesis that the phenomenon could be used to 'top-up' lubricant additive packages using a fuel treatment system. It is hypothesised that a lubricant top-up additive package could be blended with the gasoline at extremely low levels, and could help to maintain anti-oxidant and friction modifier performance of the sump lubricant by constantly adding to depleting supplies of additive, thus extending the drain interval of the sump lubricant and maintaining the efficiency of the engine.

\begin{tabular}{cccc}
\hline Test Parameter & $\begin{array}{c}\text { (Thompson, } \\
\text { Radcliffe et al. } \\
1997)\end{array}$ & $\begin{array}{c}\text { (Murakami and } \\
\text { Aihara 1991) }\end{array}$ & $\begin{array}{c}\text { Chapter 2, Section } \\
2.4 .1\end{array}$ \\
\hline Engine Type & $4-$ stroke & $4-$ stroke & $4-$ stroke \\
\hline Bore & $86 \mathrm{~mm}$ & $83 \mathrm{~mm}$ & $86 \mathrm{~mm}$ \\
\hline Stroke & $86 \mathrm{~mm}$ & $83.6 \mathrm{~mm}$ & $86 \mathrm{~mm}$ \\
\hline Fuel Type & Iso-octane & - & ULG 95 \\
\hline Oil Type & $10 \mathrm{~W} / 30$ & $10 \mathrm{~W} / 30$ & $5 \mathrm{~W} / 30$ \\
\hline Engine Load & WOT & $19.6 \mathrm{Nm}$ & $50 \%$ of full \\
\hline Speed & $1500 \mathrm{rpm}$ & $2000 \mathrm{rpm}$ & 1500 rpm \\
\hline Cylinder Liner & $70-150^{\circ} \mathrm{C}$ & $80^{\circ} \mathrm{C}$ & $\sim 90^{\circ} \mathrm{C}$ \\
Temp & Barrel Faced & - & Barrel Faced \\
\hline Top Ring & Napier Scraper & - & Napier Scraper \\
\hline Second Ring & Twin-land, Two & - & Twin-land, Two \\
\hline Oil Control Ring & Piece & & Piece \\
\hline
\end{tabular}

Table 6-1 Comparison of Sump Fuel Dilution Findings

The key information obtained from the tests showed that fuel dilution does exist in both the piston assembly lubricant and the cylinder liner oil film, in large enough quantities to significantly influence the rheology of the lubricant, affecting the lubrication conditions of the system. A potassium marker used to simulate the presence of friction modifier in the gasoline was found in all samples extracted from the piston assembly system lubricant and was found to accumulate in the region around the top of the cylinder liner and in the top piston ring groove. Increasing 
both engine speed and load was found to increase the amount of additive administered to the piston assembly lubricant.

The knowledge gained from investigation conducted in Chapter 2 was used to influence the design of the additive screening tests which are the main subject of Chapter 3. The additive screening tests were designed to scope the effectiveness of environmentally friendly friction modifier additives in the most arduous of incylinder conditions; around TDC where the boundary lubrication regime dominates. In these tests XHVI-4 ${ }^{\mathrm{TM}}$ base oil was selected to simulate the viscosity of lubricant with approximately $30 \mathrm{wt} \%$ fuel dilution, a condition found at the top ring zone when running on high boiling point gasoline. A typical TDC near surface cylinder liner temperature of $110^{\circ} \mathrm{C}$ was measured in Chapter 2, and was used as the surface temperature of the cylinder liner specimen in the additive screening tests. The load placed on the specimens was again representative of the most arduous combustion conditions measured in the engine, and helped to ensure the bench-top simulation was as similar to measured real-life conditions as was possible. The levels of Potassium marker found in the lubricant samples in Chapter 2 were found to be astonishingly high. For this reason the high treatment level of $2 \mathrm{wt} \%$ additive in base oil was selected to reflect the accumulation of additive in the lubricant, as well as to ensure a homogenous supply of additive to the working contact.

The selection of environmentally friendly friction modifier additives for screening using the bench-top test was conducted scientifically. Primarily based on the comprehensive findings of Bowden and Tabor (1950), friction modifer families and species were selected for investigation. However, in the research presented in this thesis, unlike that of Bowden and Tabor, the additives were subjected to incylinder conditions. The research conducted in Chapter 3 focused on gaining a better understanding of the friction modifier additives, allowing the formulation of an environmentally friendly additive package for testing in an engine, as presented in Chapter 5.

Similarly to the tests presented in Chapter 3 , which used a TE-77 bench top reciprocating tribometer, some work was conducted by Castle and Bovington (2003) using a High Frequency Reciprocator Rig (HFRR). In those tests boundary friction coefficients were measured for similar additives using a high-frequency reciprocating rig (HFRR). The differences between the test conditions employed by Castle and Bovington (2003) and those presented in Chapter 3 are outlined in Table 6-2.

Although some of the key test conditions such as temperature and additive concentration are relatively similar, the differences between the two sets of tests are significant. In addition to those differences outline in the above table, there are 
thought to be other differences such as component composition and geometry which can have a considerable influence on the friction measured in the system. Despite these differences a bulk comparison between the findings is still thought to be worthwhile. In order to do this, results have been normalised to read in terms of reduction in coefficient of friction, a percentage value calculated from consideration of the coefficient of friction measured with the pure base oil, and that measured with base oil containing friction modifier.

\begin{tabular}{cccccccc}
\hline Research & Test Type & $\begin{array}{c}\text { Test } \\
\text { Duration } \\
(\mathrm{hrs})\end{array}$ & $\begin{array}{c}\text { Additive } \\
\text { Conc. } \\
(\mathrm{wt} \%)\end{array}$ & $\begin{array}{c}\text { Load } \\
(\mathrm{N})\end{array}$ & $\begin{array}{c}\text { Stroke } \\
(\mathrm{mm})\end{array}$ & $\begin{array}{c}\text { Freq. } \\
(\mathrm{Hz})\end{array}$ & $\begin{array}{c}\text { Temp. } \\
\left({ }^{\circ} \mathrm{C}\right)\end{array}$ \\
\hline $\begin{array}{c}\text { Chapter 3 } \\
\text { Section } \\
3.3 .1\end{array}$ & $\begin{array}{c}\text { Ring on } \\
\text { Liner }\end{array}$ & 1 & 2 & 150 & 5 & 33 & 110 \\
\hline $\begin{array}{c}\text { Castle \& } \\
\text { Bovington }\end{array}$ & $\begin{array}{c}\text { Ball on } \\
\text { Flat }\end{array}$ & 1 & 1 & 4 & 1 & 20 & 100 \\
$(2003)$ & & & & & & & \\
\hline
\end{tabular}

Table 6-2 Comparison of Test Conditions for Additive Screening Tests

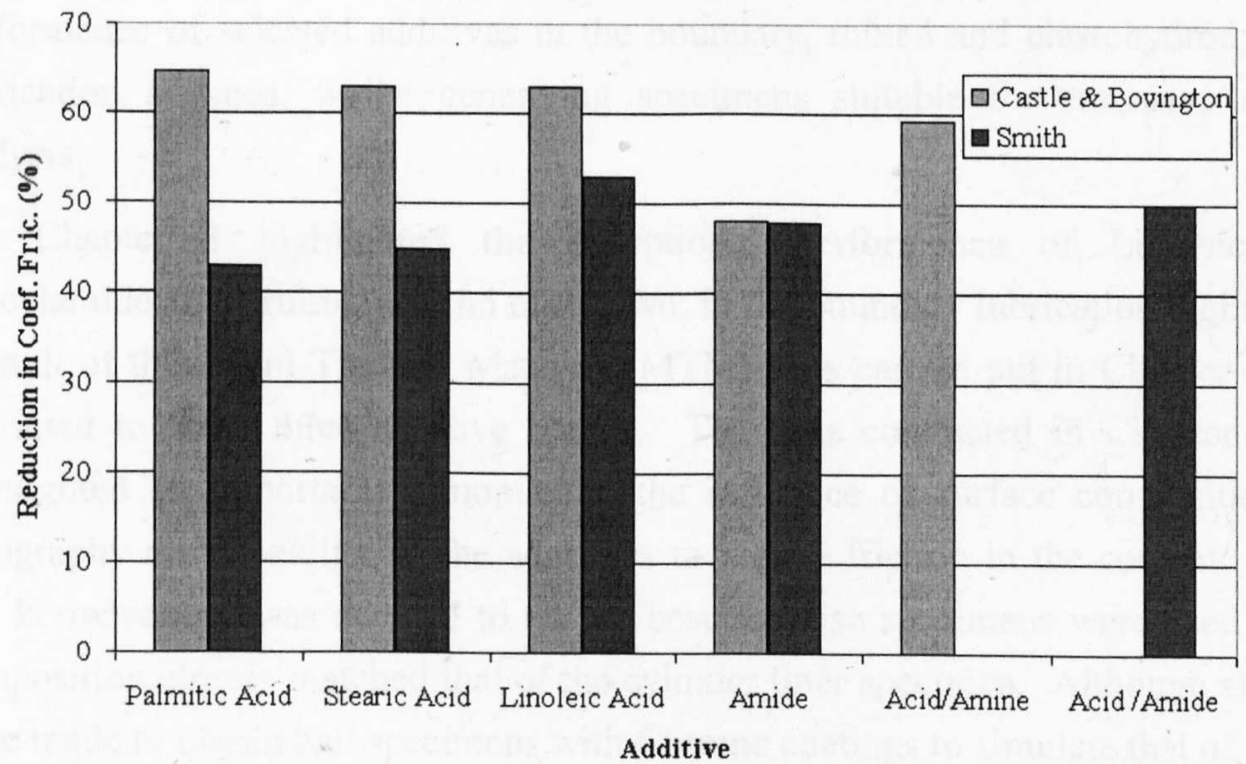

Figure 6-1 Comparison of Results Presented by Castle and Bovington (2003) and Those Presented in Chapter 3 
It is clear on initial inspection of Figure 6-1 that despite normalisation of the data sets there are significant differences between the findings of this thesis and those of Castle and Bovington (2003). This is primarily brought about by the high coefficient of friction measured by Castle and Bovington when running with pure base oil alone, 0.27 for this set of tests. However, bulk comparison of trends is possible, and shows that the friction reducing performance of the additives is significant, particularly that of Linoleic acid which performed strongly in both sets of tests. Interestingly both the tests conducted with amide based friction modifier report similar reductions in coefficient of friction, although given the difference between other data comparisons this is probably more coincidence than anything else. However, blending acid and amine, or acid and amide, both appear to have detrimental effects on the performance of the acid based additive suggesting it is more effective when employed on its own.

The conclusions made by Castle and Bovington (2003) state that findings show that boundary friction coefficients decrease with increasing chain length and higher levels of unsaturation, conclusions which this thesis further validates. However, the novel approach taken to the investigation of additives in this thesis furthers the knowledge of CHON friction modifiers by detailing the formation and removal of surface films, measuring the quality of surface films and investigating the mechanism in which surface films reduce friction.

As with the preceding chapters, the findings of Chapter 3 were used to guide the creation of tests conducted in Chapter 4, whose focus was on assessing the performance of selected additives in the boundary, mixed and elastohydrodynamic lubrication regimes, whilst generating specimens suitable for extensive surface analysis.

Chapter 3 highlighted the exceptional performance of Linoleic acid, Erucylamide and EruLin, a blend of the two, in the boundary lubrication regime. As a result of this, Mini Traction Machine (MTM) tests carried out in Chapter 4 were restricted to these three additive blends. The tests conducted in Chapter 3 also highlighted an important phenomenon, the influence of surface composition and topography on the ability of the additives to reduce friction in the contact. Given this knowledge it was decided to ensure bespoke disc specimens were used whose composition closely matched that of the cylinder liner specimen. Although attempts were made to obtain ball specimens with Chrome coatings to simulate that of the top compression ring, it was not possible to acquire samples suitable for testing. As a compromise, bearing steel balls were selected due to their high chrome composition. Unlike the specimens used in the Chapter 3 bench-top tribometer tests, the surface roughness of the specimens used in the Chapter 4 MTM tests was kept deliberately 
low to aid subsequent surface analysis. A customised test program was also developed for the MTM tests, primarily guided by findings of the TE-77 tests presented in Chapter 3, and allowed the investigation of film formation with respect to time whilst ensuring that significant additive films were created on the specimen surfaces for subsequent surface analyses.

As detailed in Chapter 4, the MTM machine provided the opportunity to scope the performance of the best performing additives of the TE-77 tests in the boundary, mixed and elastohydrodynamic lubrication regimes. However, the contact geometry and test conditions varied a great deal between the MTM and TE-77 tests as detailed in Table 6-3.

\begin{tabular}{ccccccc}
\hline Test & Test Config. & $\begin{array}{c}\text { Contact } \\
\text { Type }\end{array}$ & $\begin{array}{c}\text { Base } \\
\text { Specimen } \\
\text { Comp. }\end{array}$ & $\begin{array}{c}\text { Top } \\
\text { Specimen } \\
\text { Comp. }\end{array}$ & $\begin{array}{c}\text { Base Oil } \\
\text { Type }\end{array}$ & $\begin{array}{c}\text { Temp. } \\
\left({ }^{\circ} \mathrm{C}\right)\end{array}$ \\
\hline TE-77 & Ring / Liner & $\begin{array}{c}\text { Reciprocating } \\
\text { Pure Sliding }\end{array}$ & $\begin{array}{c}\text { Grey } \\
\text { Cast Iron }\end{array}$ & $\begin{array}{c}\text { Chrome } \\
\text { Coated }\end{array}$ & XHVI-4TM & 110 \\
\hline MTM & Ball / Disc & Slide / Roll & $\begin{array}{c}\text { Grey } \\
\text { Cast Iron }\end{array}$ & $\begin{array}{c}\text { Chrome } \\
\text { Steel } \\
\text { AISI } \\
52100\end{array}$ & $\begin{array}{c}\text { XHVI- } \\
8.2^{\mathrm{TM}}\end{array}$ & 125 \\
\hline
\end{tabular}

Table 6-3 Comparison of Key Test Parameters in TE-77 and MTM Tests

Despite the many differences between the TE-77 and MTM tests it is worthwhile to make comparison where possible, such as where the lubrication conditions are similar. As both tests investigated the performance of the additives in the boundary lubrication regime an attempt is made at the comparison of additive performance in this region, as shown in Figure 6-2. The calculation of reduction in boundary friction coefficient for the MTM tests was based on the differences between the average results for the base oil alone and those where additive was present, as presented in Figure 4-5 and Figure 4-10 respectively.

Inspection of Figure 6-2 shows some similarities between the boundary friction coefficient measurements recorded in the TE-77 tests, and those recorded during the MTM tests. Of the three additive blends tested, Erucylamide performs worst in both TE-77 and MTM tests, although the reduction in friction brought about by the additive is still considerable. The best performer in the TE-77 tests was Linoleic acid, closely followed by EruLin. For the MTM tests the reciprocal of this relationship is shown, with EruLin performing best. 


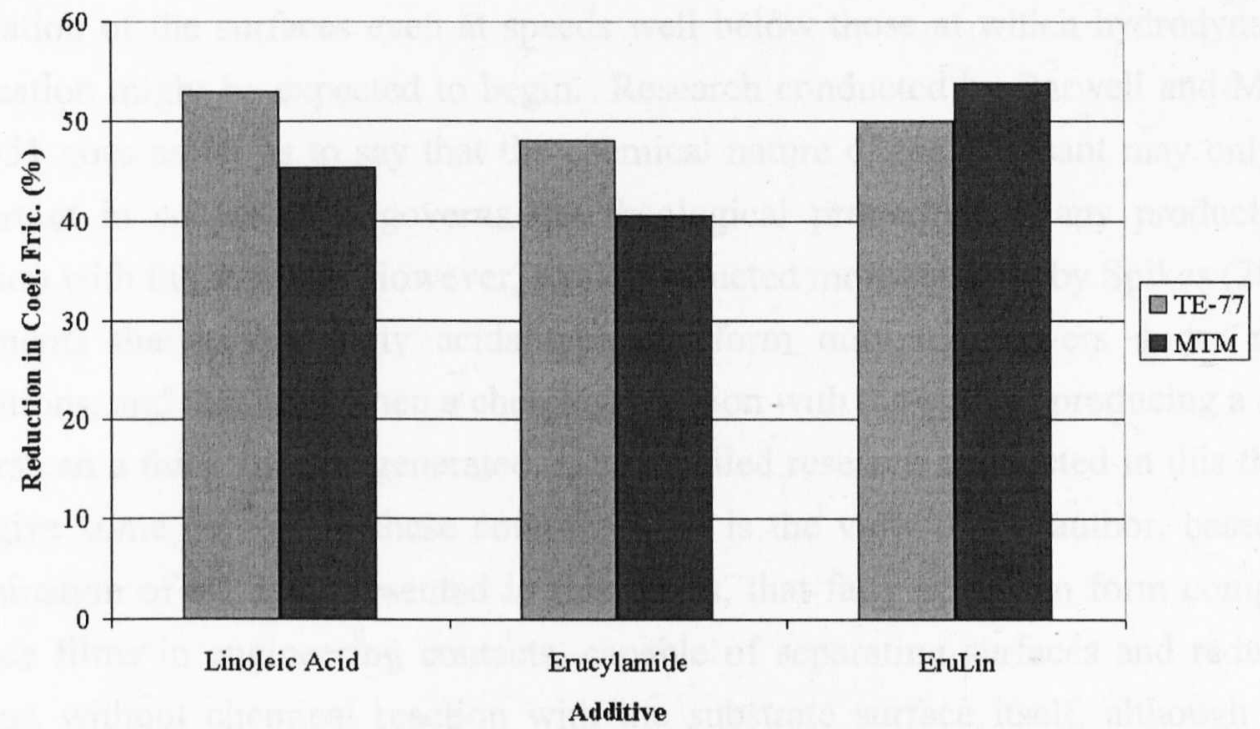

Figure 6-2 Comparison of Boundary Friction Coefficient for TE77 and MTM Tests

It is discussed in Chapter 5 that considerable energy savings can be made in the engine if a friction modifier additive capable of reducing friction in the mixed lubrication regime is successfully employed. MTM tests which investigated the performance of Linoleic acid, Erucylamide and EruLin highlighted the effectiveness of the fatty acid molecule at reducing friction in the mixed regime. Erucylamide was shown to have comparatively little effect in the mixed lubrication regime, although when blended with Linoleic acid, as EruLin, some slight improvement on the performance of the acid alone was seen, Figure 4-10. However, the presence of the amide was shown to be damaging to the cast iron surface, resulting in considerable corrosive wear. For this reason Linoleic acid was selected for use in Chapter 5, 'Novel Fuel Formulation Engine Tests'.

In addition to aiding the selection of additive for use in Chapter 5, the MTM tests conducted in Chapter 4 served to create samples suitable for detailed surface analyses using optical microscopy, profilometry, FTIR microscopy, SEM / EDX and XPS techniques. The direct analysis of surface films found on the specimens after the test sequence is in many respects a seminal piece of work. The surface analyses were conducted to further the understanding of the way in which $\mathrm{CHON}$ friction modifiers reduce friction in contacts, a mechanism which has been a point of contention between researchers for many years, as described in Chapter 1. Many researchers have commented on the friction reduction mechanism of films created through application of fatty acid friction modifier, most concluding on the formation of a thick additive film. This thick, or viscous, film theory was discussed by Bowden and Tabor (1950) where they observed that the formation of a metallic soap can create a thick viscous layer between sliding surfaces leading to relatively large 
separation of the surfaces even at speeds well below those at which hydrodynamic lubrication might be expected to begin. Research conducted by Barwell and Milne in 1951 goes as far as to say that the chemical nature of the lubricant may only be important in so far as it governs the rheological properties of any products of reaction with the surface. However, work conducted more recently by Spikes (2002) comments that simple fatty acids typically form only monolayers under most conditions, and that only when a chemical reaction with the surface producing a soap occurs can a thick film be generated. The detailed research conducted in this thesis can give some context to these comments. It is the view of the author, based on examination of all data presented in this thesis, that fatty acids can form complete surface films in engineering contacts, capable of separating surfaces and reducing friction without chemical reaction with the substrate surface itself, although it is thought that given the correct conditions, some level of chemical reaction can occur. Where chemical reaction of the fatty acid occurs it does not transform the surface of the bounding solid (like ZDDP) but rather takes elements from it to create a metallic soap. This phenomenon is thought to be heavily influenced by the composition of the surface with which it reacts. It is hypothesised, although no hard evidence exists, that fatty acids can form thick, multi-layer, films during mixed lubrication conditions which can significantly reduce contact friction. Similarly amide friction modifiers are thought to be capable of forming multi-layer surface films, although there is more substantial evidence to suggest their reaction with the surface is mostly chemical rather than physical.

Given the exceptional performance of Linoleic acid in both TE-77 (Chapter 3) and MTM tests (Chapter 4) the friction modifier additive was chosen for testing in a set of engine tests described in Chapter 5. Engine tests were designed to accurately measure the power increase as a result of switching from standard gasoline to gasoline containing friction modifier. Results of these tests showed an increase in the brake power of the engine in the region of $2 \%$ when running on friction modified gasoline. Interestingly the effect was not seen to be immediately reversed on switching back to standard gasoline. Subsequent analysis of the sump lubricant showed small quantities of additive, although not in substantial enough quantities to have had a significant effect on the friction within the system. Based on this information it was concluded that either the tenacity of the additive films was greater than that which was measured in the bench-top tests, or more probably that a significant accumulation of additive occurred in the lubricant film on both the piston assembly and cylinder liner and continued to effect the piston assembly lubrication after the supply of gasoline containing friction modifier was removed. 
As neither of the bench-top tribometer simulations attempted to simulate all incylinder conditions experienced by the piston assembly, it is not valid, nor was it ever the intention, to look at the bench-top results as a means by which to predict the exact performance of the additive in the engine. However, some comparison can be made at the point of stroke reversal between the TE-77 reciprocator ring on liner tests and the reversal point of the entire piston assembly as measured in the engine tests. Conditions used in the TE-77 tests were chosen to simulate the operation of the top compression ring near the top dead centre after ignition, so comparison with engine data captured at a similar point is a valid exercise.

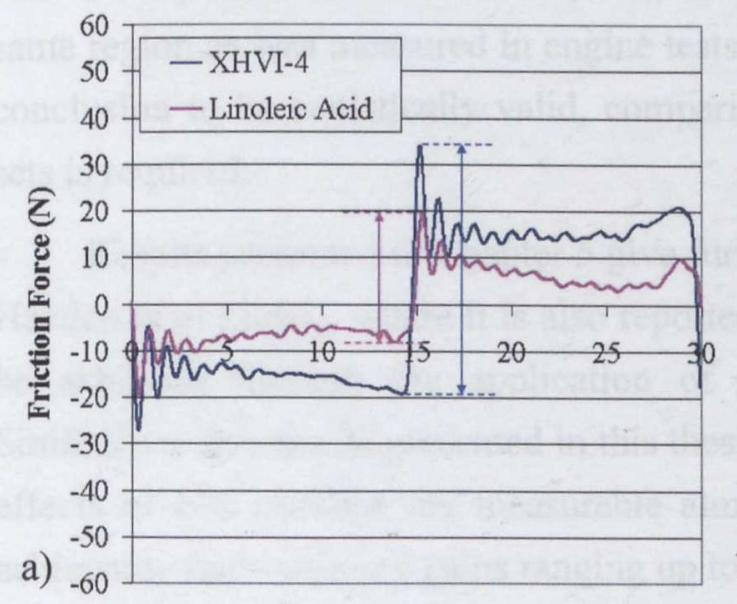

Time (ms)

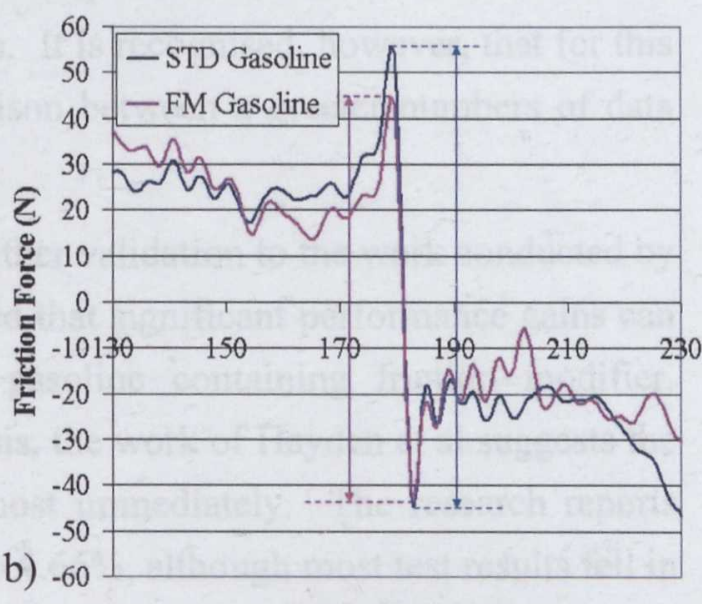

Crank Angle (deg)

Figure 6-3 Comparison of End-of-Stroke Reversal Between a) TE-77 and b) Engine Tests

Initial inspection of Figure 6-3 shows that peak friction measured at reversal is considerably greater in the engine tests than was measured in the TE-77 tests, not surprising as the measurement is for the full piston assembly, not just the top compression ring, as was the case in the bench-top test. Modelling work conducted by Mufti (2004) simulated the same piston assembly in the same engine with similar operating conditions, although at $800 \mathrm{rpm}$ rather than $2000 \mathrm{rpm}$ which was the engine speed for tests conducted in Chapter 5. Mufti estimated the reversal friction to be approximately $60 \mathrm{~N}$ at this point in the cycle, a value which is in relatively good agreement with the results presented in Figure 6-3b.

To allow comparison between the bench-top and engine tests the reduction of stroke reversal friction between the non additive and friction modified trace was calculated between the limits indicated on the diagram in Figure 6-3. For the TE-77 tests, Figure 6-3a, the addition of Linoleic acid to the base oil reduced the reversal friction by $47 \%$. For the engine test, Figure 6-3b, the presence of Linoleic acid in 
the fuel reduced the reversal friction by just approximately $13 \%$. However, the simulations conducted by Mufti (2004) suggest that the top compression ring, operating under similar conditions, is responsible for approximately $32 \%$ of the overall piston assembly friction. If it is assumed that at this point in the engine cycle the only ring whose profile and lubrication condition allow its friction to be influenced by the presence of friction modifier additive was the top compression ring, a reduction of $47 \%$ for the top ring friction would reduce the total piston assembly friction by approximately $15 \%$. Thus it can be suggested that there is some scientifically based correlation between the performance of the friction modifier additive in the bench-top test, which was designed to simulate in-cylinder conditions near the top dead centre reversal point, and the performance of the additive in the same region as was measured in engine tests. It is recognised, however, that for this conclusion to be statistically valid, comparison between a greater numbers of data sets is required.

Results presented in Chapter 5 give further validation to the work conducted by Hayden et al (2001), where it is also reported that significant performance gains can be achieved through the application of gasoline containing friction modifier. Similarly to the results presented in this thesis, the work of Hayden et al suggests the effects of FM gasoline are measurable almost immediately. The research reports achievable fuel economy gains ranging up to $4.66 \%$, although most test results fell in the region of $2 \%$. Achievable fuel economy gains were shown to be heavily influenced by the initial treatment level of the gasoline, the temperature of the engine in which it was employed as well as the vehicle. Therefore the typical fuel economy gain of approximately $2 \%$ measured as a result of application of FM gasoline in this project looks to be in good agreement with that of Hayden et al (2001).

\subsubsection{The Success of FM Gasoline}

In Chapter 1 it was stated that in the 1970's it was thought that there were really only two ways in which a gasoline additive could increase the fuel economy of the vehicle; by increasing the calorific value of the fuel or by increasing the efficiency with which the chemical energy of the fuel is converted to useful work. Work conducted in this project broadens the interpretation of the latter mechanism by suggesting that increases in fuel economy can be achieved through increasing the efficiency with which the kinetic energy of the piston is converted to useful work. Of the eight listed views presented in Chapter 1, Section 1.5, commenting on claims made by inventors of fuel additives that can improve economy (Robinson 1977), the success of FM gasoline reported in this project relates best to numbers 2, 5 and 8 as reviewed below: 
2. It will, if it is valid, probably result in small benefit (say $5 \%$ at most) compared with benefits from mechanical modifications.

5. It may have deleterious side effects both in the short and long term.

8. It might indeed give a real benefit by some as yet poorly realized and understood mechanism.

The first of the viewpoints presented above is, in the view of the author, extremely naive as a $5 \%$, or $2 \%$ (as is the case in this study), increase in vehicle fuel economy is a substantial saving when considered with a global perspective, as further explained in the following section.

Point 5 above states there may be deleterious side effects associated with the application of the gasoline additive, as was the case in this study. In Chapter 5, section 5.1.3, it was stated that the use of FM gasoline might have contributed to deposits on, and the fouling of, engine components. However, as tests were conducted in absence of both fuel and lubricant additive package, both of which would usually contain a detergent, it is possible to suggest that this fouling may not be a significant factor. Another deleterious effect of the implementation of Linoleic acid as a gasoline friction modifier is the fact that it is an unsaturated molecule. As was discussed in Chapter 3, section 3.7.1, having polyunsaturated, or indeed unsaturated, molecules in the sump lubricant is undesirable as they are prone to cross linking, and as such would be likely to form sludge degrading the performance of the oil. However, it is also necessary to note that should the molecules employed be saturated, there should be no degrading effect on the engine lubricant, and their performance is thought to be only marginally poorer than their unsaturated equivalents.

Point 8 shown above states that fuel economy benefits can sometimes be achieved through poorly realised and understood mechanisms. This thesis presents a vast quantity of research on environmentally friendly or ' $\mathrm{CHON}$ ' friction modifiers and the mechanism through which they can reduce engine friction and increase fuel economy. This thesis adds greatly to the understanding of $\mathrm{CHON}$ friction modifiers and presents tests which realise the capabilities of such technologies. However, work is still needed to be done in the area to completely understand the complex workings of organic friction modifiers, much of which is listed in the final chapter of this thesis. 


\subsection{The Global Impact Of The Research}

It was stated in Chapter 1 that the driving force behind the need for research into technologies which increase vehicle fuel economy was the recognition of society that the world needs to use its resources more efficiently. As well as reducing the rate of consumption of limited oil reserves, a reduction in emissions created through the burning of hydrocarbon products is also needed. In response to the needs of society, this project focussed on the development of a technology which has the power to reduce the gasoline consumption of the motor vehicle, which inherently slows the rate of consumption of hydrocarbon based fuels and consequently reduces the amount of vehicle emissions, but also acts to maintain the efficiency of the catalytic converter, a device essential to controlling exhaust emissions.

On completion of this research project it is not enough to simply state that gasoline administered friction modifier can work to both reduce gasoline consumption and limit exhaust emissions, it is necessary to relate the success of technologies such as CHON FM gasoline to the potential global impact of such products. This section assesses the impact of successful fuel economy saving technologies based on the findings of this research project and statistics which detail the way in which we function as a society in the UK.

Figure 6-4 presents the gasoline consumption in the UK over the past ten years. Each year since 1996 the consumption of gasoline in the UK has decreased; this is as a result of many factors such as advances in vehicle design, engine design, combustion technology as well as advances in fuel and lubricant technology. However, it is thought that the most influential factor has been the consumer's choice of diesel powered vehicles, which gain popularity every year.

To assess the impact on gasoline consumption in the UK as a result of successful implementation of FM gasoline, a number of assumptions need to be made. If it is considered that during 2005 there were no further advances in vehicle technologies, and the amount of gasoline and diesel powered vehicles remained constant, the gasoline consumption saving in the UK in 2006 achievable solely as a result of successful implementation of FM gasoline would be as represented by the final data point (red triangle) shown on Figure 6-4. 


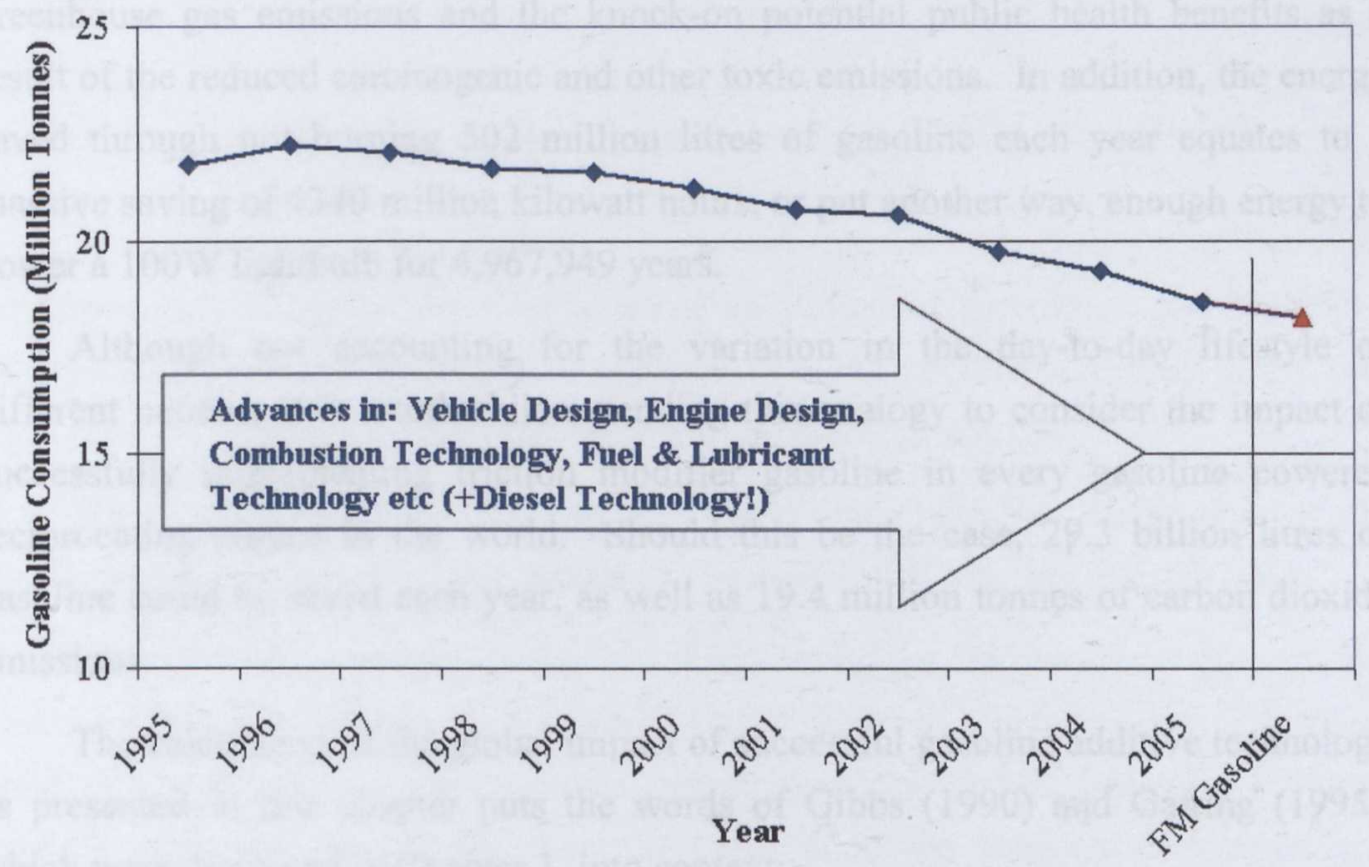

Figure 6-4 Gasoline Consumption in the UK and Calculated Saving Made Through Implementation of Friction Modified Gasoline. After (DfT 2006)

Contrary to the belief of Robinson in 1977, a small energy saving made through the application of a gasoline additive can have a significant effect which rivals that of mechanical modification. Yearly savings have been calculated based on the research conducted in this project and statistical data detailing the day-to-day lifestyle of the UK resident in 2005. The following lists the hypothetical annual savings which could be made as a result of the application of FM gasoline in the UK:

- 502 million litres of gasoline

- $£ 160$ for the motorist (or 1233 miles free, or more power!)

- 388000 tonnes carbon $\left(\mathrm{CO}_{2}\right.$ emissions)

- 23420 tonnes carbon (CO emissions)

- 4900 tonnes nitrogen (NO emissions)

- 138 tonnes particulates (PM10)

- 58 tonnes benzene

- 18 tonnes butadiene

- 48 tonnes sulphur dioxide

Savings in gasoline consumption of the internal combustion engine results in financial savings for the consumer, as well as environmental savings due to reduced 
greenhouse gas emissions and the knock-on potential public health benefits as a result of the reduced carcinogenic and other toxic emissions. In addition, the energy saved through not burning 502 million litres of gasoline each year equates to a massive saving of 4340 million kilowatt hours, or put another way, enough energy to power a $100 \mathrm{~W}$ lightbulb for $4,967,949$ years.

Although not accounting for the variation in the day-to-day lifestyle of different nations, it is worthwhile extending this analogy to consider the impact of successfully implementing friction modifier gasoline in every gasoline powered reciprocating engine in the world. Should this be the case, 29.3 billion litres of gasoline could be saved each year, as well as 19.4 million tonnes of carbon dioxide emissions.

The calculation of the global impact of successful gasoline additive technology as presented in this chapter puts the words of Gibbs (1990) and Gairing (1995), which were discussed in Chapter 1, into context;

"Today's additive technology can improve the quality of any gasoline tremendously. Given the right commitment, careful selection of the right additives, and the right follow through, the end result will be a superb product."

Lewis M. Gibbs (1990), Consulting Engineer at Chevron Oronite.

"Fuel additives are like alloy agents in metal, they are both essential and indispensable."

Max Gairing (1995), Senior Manager of Operational Fluids at Mercedes-Benz. 


\section{Conclusions \& Recommendations for Future Work}

The purpose of this chapter is to present the final conclusions of the work described in this thesis, drawing together all points of discussion and summary. Conclusions presented consider all areas of research conducted in this thesis, and have been organised into a priority sequence, most important first.

Recommendations for future work are also made based on the findings of this thesis, detailing areas which are thought to be essential for further investigation.

The need for technologies capable of increasing the fuel economy of the motor vehicle is becoming desperate, the health of the planet in which we live, indeed our children's world, relies heavily on the advances made through research. Research such as that presented in this thesis is essential to the progress of technologies capable of significantly reducing hydrocarbon consumption and exhaust emissions which harm our environment.

The originality of this thesis comes from the approach taken when tackling the main aim; furthering the fuel economy of the gasoline engine. At every step of the way, scientific knowledge disseminated from the research of others was considered logically and laterally forming a sound base from which innovation could be used to further understanding.

\subsection{Conclusions}

- The hypothetical impact of research which increases fuel economy, such as is presented in this thesis, was illustrated by calculations detailing the effects as seen by both the motorist and society in general. Based on the research conducted in this project and statistical data detailing the day-to-day lifestyle of the UK resident in 2005 , the following key yearly savings were calculated:

- 502 million litres of gasoline

- $£ 160$ for the motorist (or 1233 miles free, or more power)

- 388,000 tonnes of carbon (from $\mathrm{CO}_{2}$ )

- In-cylinder lubricant sampling techniques were both created and developed to gain a better understanding of the phenomenon of fuel contamination in the piston assembly lubricant, and provided a means 
by which to assess the possibility of administering friction modifier to the piston assembly lubricant via gasoline.

- Experiments undertaken at a range of engine operating conditions showed fuel dilution to be a prevalent phenomenon in both the top ring zone and cylinder liner oil film. Typical fuel dilutions in the top ring zone ranged from $5-11 \mathrm{wt} \%$, whereas those of the cylinder liner ranged from $0.2-3.6 \mathrm{wt} \%$. However, the final boiling point of the fuel was shown to be the most significant factor influencing the fuel dilution of the piston assembly lubricant. The measured levels of dilution were shown to be significant enough to affect the viscosity of the lubricant, heavily influencing the lubrication of the piston assembly.

- Research leads to the conclusion that the transport of fuel from the combustion chamber to the engine sump takes place via one of two mechanisms; 1) fuel passes the piston rings in the blow-by gases in a vapour phase, 2) fuel adsorbs into the cylinder liner lubricant, which is returned to the sump in a liquid phase through the natural action of the piston assembly. These mechanisms were also shown to transport gasoline additive to the sump, fathering the hypothesis that the system could be used to 'top-up' the sump lubricant additive package with fresh supplies of chemicals, extending the drain interval of the oil.

- Two categories of lubricant residence times have been hypothesised, one associated directly with the lubrication of the piston assembly, and one the cylinder liner. The residence time for the piston assembly is thought to be considerably longer than that of the cylinder liner, allowing the accumulation of large amounts of additive administered via the gasoline. Accumulation of additive also takes place in the cylinder liner oil film, although in smaller quantities.

- A bench-top test was developed to rapidly screen the performance of environmentally friendly gasoline friction modifiers under arduous incylinder boundary lubrication conditions using sections of engine components in a TE-77 tribometer. The test proved capable of generating a vast amount of information relating to the films created by the candidate friction modifiers, including their formation and removal as well as the mechanism of their action.

- Results of the TE-77 bench-top screening tests showed fatty acids to be effective friction modifier additives capable of successful operation in arduous boundary lubrication conditions. The findings suggest that the 
friction reducing performance of fatty acid molecules increases with increasing hydrocarbon chain length and unsaturation level. Linoleic acid was shown to perform best and was found to be capable of reducing the boundary friction coefficient by $53 \%$.

- Tests showed some evidence to support the hypothesis that the combination of two carefully selected friction modifier additives in an additive package can lead to the creation of more effective friction modifier films, capable of reducing the boundary friction coefficient further than that achievable by either of the additives on their own.

- Given that piston rings experience multiple lubrication regimes in each engine cycle, a series of tests were conducted using a Mini Traction Machine (MTM) which tested the effectiveness of selected friction modifier additives in multiple lubrication regimes. The tests also allowed investigation into the effects of friction modifiers on specimen wear. The presence of Linoleic acid in base oil was shown to reduce specimen wear during MTM tests by approximately 38\% when compared with the result for the base oil alone. The presence of amide friction modifier was shown to increase specimen wear by approximately $85 \%$ compared to the base oil result, as such its action is thought to be corrosive.

- In tests conducted in a Mini Traction Machine, Linoleic acid was shown to significantly reduce the coefficient of friction in both the mixed and boundary lubrication regimes, as much as $45 \%$ in the latter when compared with the result for base oil alone. The combination of Linoleic acid and Erucylamide was shown to be the best performing additive blend in these tests and reduced the boundary friction coefficient by approximately $54 \%$.

- Surface analyses conducted on specimens operated in lubrication systems containing fatty acid and/or amide friction modifiers showed evidence of film formation. Given the arduous specimen cleaning procedure employed prior to XPS analyses, it seems wrong to suggest the detected surface films were merely physically attached to the surface, yet hard evidence was not found to suggest the presence of dense or robust chemical films.

- Based on the findings of this research it is thought that fatty acids can form complete surface films in engineering contacts, capable of separating surfaces and reducing friction without chemical reaction 
with the substrate surface itself, although it is thought that given the correct conditions, some level of chemical reaction can occur. Where chemical reaction of the fatty acid occurs it does not transform the surface of the bounding solid (like ZDDP) but rather takes elements from it to create a metallic soap. This phenomenon is thought to be heavily influenced by the composition of the surface with which it reacts. A monolayer of complete and densely packed film of fatty acid is thought to be capable of effectively reducing contact friction where conditions are arduous. Multi-layer films of fatty acid molecules are thought to form where lubricating conditions allow and may act like a viscous layer between sliding surfaces.

- Gasoline containing friction modifier was shown to immediately reduce piston assembly friction through modification of the lubrication conditions in both the boundary and mixed lubrication regimes. The effects of gasoline administered friction modifier were not shown to be immediately reversible with the supply removed, suggesting accumulation of the friction modifier species in the piston assembly lubrication system continues to reduce friction.

- Engine power was shown to increase by approximately $2 \%$ through the application of gasoline administered Linoleic acid friction modifier. Tests conducted in a single-cylinder firing gasoline engine using an instantaneous mean effective pressure total friction measurement system showed piston assembly friction was significantly reduced as a result of the gasoline administered friction modifier.

\subsection{Recommendations for Future Work}

The following are areas identified for either further work or future research, the former detailing small increments to this study which were not possible due to time constraints, the latter detailing new areas of research guided by the findings of this research project.

Despite advances in technologies which are less dependant on hydrocarbon fuels, the hydrocarbon powered vehicle is likely to be around for many years to come. It is not therefore an exaggeration to suggest that the future of the planet depends on advances in motor vehicle technology. Future work on technologies which can further the fuel economy of the motor vehicle is therefore essential. 


\subsubsection{Further Work}

- Work should be conducted to test the performance of the Three Point Sampling (TPS) system at speeds in excess of $3000 \mathrm{rpm}$ at full load. The system may provide a unique opportunity to gain understanding of the piston assembly lubrication of a gasoline engine at high speed conditions, something not thought to have been achieved before.

- The Three Point Sampling (TPS) system should be developed to include a bespoke mechanism for clearing, or resisting the creation of, deposits in the sampling pipes. This would lead to greater efficiency of the system.

- The additive screening test developed in this thesis should be conducted on a long chain $\left(\mathrm{C}_{22}\right.$ or greater) polyunsaturated amine friction modifier. Once sourced, the hypothesis made in this thesis that films formed by this chemical would result in exceptionally low coefficients of sliding friction could be tested.

- Tests should be conducted to investigate the interesting effects seen when performing additive screening tests using molybdenum flame sprayed coated piston rings. It is thought that effects seen may have been as a result of either the coating material itself or the rough topography of the ring. These variables need decoupling in order to further understand the interesting phenomena.

- The high speed dynamic mapping of the response of the strain gauge equipped connecting rod as it passes through the zero-load state should be conducted. Information taken from this test could then be incorporated in the post processing program to map the measured voltage with the true force experienced by the connecting rod, thus increasing the accuracy of the system.

\subsubsection{Future Research}

- Due to the continual increase in the popularity of the diesel powered vehicle it is suggested further work be conducted to assess the effectiveness and impact of diesel containing friction modifier on engine friction.

- A laboratory technique should be developed to allow the accurate quantification of friction modifier concentration in lubricant samples extracted during in-cylinder sampling firing tests. This would allow incylinder lubricant sampling tests to be conducted when firing with 
friction modified gasoline as opposed to a marker simulating friction modifier.

- Further research is needed to understand the nature of the films formed through the application of fatty acid friction modifiers. The development and application of in-depth surface analysis conducted on engineering surfaces extracted from real systems should help to explain the exact mechanism of friction reduction as brought about by fatty acid films.

- Investigate the performance of fatty acid friction modifiers outside model conditions, in systems containing fully formulated fuels and lubricants. Information gathered from these tests would give further quantification to the impact of the addition of fatty acid to an additive package in terms of friction reduction, engine cleanliness and sump lubricant contamination and degradation.

- Contact potential measurements taken between an electrically isolated top compression ring and the cylinder liner during firing engine conditions would allow the direct investigation into the effects of insitu friction modifier film formation on the lubrication condition of the top compression ring, thus aiding the development of successful additive packages.

- Development of an in-situ method to analyse the formation of organic friction modifier films on cylinder liner and piston ring surfaces in the engine, perhaps using FTIR techniques, would give invaluable information about film formation under firing conditions.

- The combustion related effects of gasoline containing friction modifier are thought to be of interest. Large treatment levels of friction modifier were used in this research and were thought to have some effect on the combustion characteristics of the engine. Investigation into these effects should help further assessment of the impact of the technology and help to identify the level of friction modifier necessary for friction reduction, whilst not significantly deteriorating combustion.

- Direct measurement of exhaust emissions when running on friction modified gasoline is thought to be necessary in order to fully assess the true environmental impact of the technology. 


\section{References}

(ATC), T.T.C. o. P.A.M. i. E. (1993). "Lubricant Additives and The Environment."

Anghel, V., C. Bovington, et al. (1999). "Thick-Boundary-Film Formation by Friction Modifier Additives." Lubrication Science 11(4): 313-335.

Aruna, M. A. and M. F. Fox (2004). "Differentiation of Lubricant Degradation in the Piston Ring Groove and Crown Land by Multivariate Data Analysis." Proceedings of the Institute of Mechanical Engineers 218(J): 1503-1511.

B.P. (2005). "Statistical Review of World Energy." B.P. Online (www.bp.com).

Bailey, G. (2003). Refinery Fuel Additives. Additives 2005, Octel.

Bair, S. and F. Qureshi (2003). "The High Pressure Rheology of Polymer-Oil Solutions." Tribology International 36: 637-645.

Bardasz, E. A. (2005). Future Engine Fluids Technologies: Durable, Fuel-Efficient, and Emissions-Friendly. 11th Diesel Engine Emissions Reduction Conference, Chicago, III.

Barnes, A. M., K. D. Bartle, et al. (2001). "A Review of Zinc Dialklydithiophosphates (ZDDPS): Characterisation and Role in the Lubricating Oil." Tribology International 34: 398-395.

Barrell, D. J. W. (2004). "Top Ring Zone Lubricant Sampling and Engine Friction Project " Shell Internal

Barrell, D. J. W., M. Priest, et al. (2004). "Experimental Simulation of Impact and Sliding Wear in the Top Piston Ring Groove of a Gasoline Engine." Proceedings of the Institute of Mechanical Engineers 218(J): 173-183.

Barwell, F. T. and B. A. Milne (1951). "The Lubrication of Rough Steel Surfaces by a Series of Metallic Soaps." British Journal of Applied Physics 2: 44-48.

Batt, R. J., J. A. McMillan, et al. (1996). "Lubricity Additives - Performance and No-Harm Effects in Low Sulfur Fuels." SAE 961943. 
BBC News and S. Mulvey (2006). "EU car CO2 Fight Only Beginning." www.bbc.co.uk/news.

Beltzer, M. and S. Jahanmir (1988). "Effect of Additive Molecular Structure on Friction." Lubrication Science 1(1): 3-26.

Blau, P. J. (2005). "On the Nature of Running-in." Tribology International 38: 10071012.

Bowden, F. P. and D. Tabor (1950). The Friction and Lubrication of Solids. Oxford, Oxford University Press.

Bowden, F. P. and D. Tabor (1964). The Friction and Lubrication of Solids. Oxford, Oxford University Press.

Braithwaite, E. R. and A. B. Greene (1978). "A Critical Analysis of the Performance of Molybdenum Compounds in Motor Vehicles." Wear 46: 405-432.

Burnett, P. J. (1992). "Relationship Between Oil Consumption, Deposit Formation and Piston Ring Motion for Single Cylinder Diesel Engines." SAE 920089.

Burnett, P. J. (1993). "Characterisation of the Ring Pack Lubricant and its Environment." Shell Internal.

Burnett, P. J. (1993). "Characterisation of the ring pack lubricant and its environment."

Bush, G. P., M. F. Fox, et al. (1991). "Composition of Lubricating Oil in the Upper Ring Zone of an Internal Combustion Engine." Tribology International 24(4): 231233.

Castle, R. C. and C. H. Bovington (2003). "The Behaviour of Friction Modifiers Under Boundary and Mixed EHD Conditions." Lubrication Science 15(3): 253-263.

Cheng, W. K., D. Hamrin, et al. (1993). "An Overview of Hydrocarbon Emissions Mechanisms in Spark-Ignition Engines." SAE 932708. 
Chibber, V. K., R. B. Chaudhary, et al. (2006). "Anti Wear and Anti Friction Characteristics of Tribochemical Films of Alkyl Octa-decanoates and their Derivatives." Lubrication Science 18: 63-76.

Clarke, D. G., I. Sherrington, et al. (1991). Simultaneous Measurement of Piston / Piston Ring Friction and Oil Film Thickness in an I C Engine.

Colgan, T. and J. C. Bell (1989). "A Predictive Model for Wear In Automotive Valve Train Systems." $\underline{\text { SAE } 892145 .}$

Cook, S. (2003). Fuel Borne Catalysts (FBC) for Diesel Particulate Filter (DPF) Regeneration. OCTEL, OCTEL.

Costello, M. T. and I. I. Riff (2005). "Study of Hydroforming Lubricants with Overbased Sulfonates and Friction Modifiers." Tribology Letters 20(3-4): 201-208.

Coy, R. C. (1991). "Engine Lubrication - Art or Science." Tribology Series 18(Leeds Lyon 17): 407-417.

Dai, W., E. Newman, et al. (1996). "Predictions of In-Cylinder Tumble Flow and Combustion in SI Engines with a Quasi-Dimensional Model." SAE 961962.

Dallas, T. and K. Dasgupta (2004). "Light at the End of the Tunnel: Recent Analytical Applications of Liquid-Core Waveguides." Trends in Analytical Chemistry 23(5): 1-8.

Danilov, A. M. (2001). "Fuel Additives: Evolution and Use In 1996-2000." A Review: Chemistry and Technology of Fuels and Oils 37(6): 444-455.

Das, S., T. Perry, et al. (2006). "Effect of Surface Etching on the Lubricated Sliding Wear of an Eutectic Aluminium - Silicon Alloy." Tribology Letters 21(3): 193-204.

Davis, F. A. and T. S. Eyre (1990). "The Effect of Friction Modifier on Piston Ring and Cylinder Bore Friction and Wear." Tribology International 23(3).

De Barros Bouchet, M. I., J. M. Martin, et al. (2005). "Mechanisms of MoS2 Formation by MoDTC in Presence of ZnDTP: Effect of Oxidative Degradation." Wear 258: 1643-1650. 
De Barros Bouchet, M. I., J. M. Martin, et al. (2005). "Boundary Lubrication Mechanisms of Carbon Coatings by MoDTC and ZDDP Additives." Tribology International 38: 257-264.

De Barros Bouchet, M. I., I. Raoult, et al. (2003). "Friction Reduction by Metal Sulfides in Boundary Lubrication Studied by XPS and XANES Analyses." Wear 254: 863-870.

Dearlove, J. and W. K. Cheng (1995). "Simultaneous Piston Ring Friction and Oil Film Thickness Measurements in a Reciprocating Test." $\underline{\text { SAE } 952470 .}$

Devaprakasam, D., O. P. Khatri, et al. (2005). "Boundary Lubrication Additives for Aluminium: A Journey from Nano to Macrotribology." Tribology International 38: 1022-1034.

Devlin, M. T., W. Y. Lam, et al. (1998). "Critical Oil Physical Properties that Control the Fuel Economy Performance of General Motors Vehicles." SAE 982503.

DfT (2006). "Transport Statistics Great Britain."

Dohner, B. R. and M. A. Wilk (1995). "Formulating for ILSAC GF-2 - Part 2: Obtaining Fuel Economy Enhancement From a Motor Oil in a Modern Low Friction Engine." SAE 95343.

Dowson, D. (1996). Friction Modelling for Internal Combustion Engines, Institute of Tribology: 301-318.

Dowson, D. (1999). "The Role of the Interface and Surface Layers in the Thin Film and Boundary Regime." Tribology Series 36: 611-619.

Dunaevsky, V. and B. Vick (2004). "Friction Temperature of the Piston Rings with Consideration of the Cylinder Wall Thickness." $\underline{\text { SAE 2004-01-0612. }}$

Duveneck, G. L., M. A. Bopp, et al. (2001). "Evanescent-Field-Induced Two-Photon Flourescence: Exitation of Macroscopic Areas of Planar Waveguides." Applied Physics B 73: 869-871. 
Fansier, T. D., D. T. French, et al. (1995). "Fuel Distribution in a Firing Direct Injection Spark-Ignition Engine Using Laser-Induced Fluorescence Imaging." $\underline{\text { SAE }}$ 950110.

Finch, G. I. and H. Wilman (1937). "Study of Surface Structure by Electron Diffraction." Ergebn. exakt. Naturwiss 16: 353-436.

Floch, A., J. V. Frank, et al. (1995). "Comparison of the Effects of Intake-Generated Swirl and Tumble on Turbulence Characteristics in a 4-Valve Engine." SAE 952457.

Fox, M. F., D. J. Picken, et al. (1997). "Paramagnetic Species in I/C Engine Top Ring Zone Lubricant Samples." Tribology International 30(6): 417-422.

Frewing, J. J. (1944). "The Heat of Adsorption of Long-Chain Compounds and their Effect on Boundary Lubrication." Proceedings of the Royal Society of London. Series A, Mathematical and Physical Sciences 182(990): 270-285.

Frolund, K., J. Schramm, et al. (1997). "An Investigation of the Cylinder Wall Oil Film Development During Warm-Up of an SI Engine Using Laser Induced Flourescence." SAE 971699.

Frottier, V., J. B. Heywood, et al. (1996). "Measurement of Gasoline Absorption into Engine Lubricating Oil." SAE 961229.

Fujita, H. and H. Spikes (2004). "The Formation of Zinc Dithiophosphate Antiwear. Films." Proceedings of the Institute of Mechanical Engineers 218(J): 265-277.

Furey, M. J. (1961). "Metallic Contact and Friction Between Sliding Surfaces." ASLE Transactions 4: 1-11.

Gacoin, E., C. Fretigny, et al. (2006). "Measurement of the Mechanical Properties of Thin Films Mechanically Confined within Contacts." Tribology Letters 21(3): 245252.

Gairing, M. (1995). "Mercedez-Benz views on Fuel and Lubricant Additives." Industrial Lubrication and Tribology 47(3): 3.

Gamble, R. J. (2002a). The Influence of Lubrication Degradation on Piston Assembly Tribology. Mechanical Engineering. Leeds, The University of Leeds: 241. 
Gamble, R. J. (2002b). "Detailed Analysis of Oil Transport in the Piston Assembly of a Gasoline Engine." Tribology Letters 14(2): 147-156.

Gangopadhyay, A. K., E. Soltis, et al. (2004). "Valvetrain Friction and Wear: Influence of Surface Engineering and Lubricants." Proceedings of the Institute of Mechanical Engineers 218(J): 147-156.

Gangopadhyay, A. K., J. Sorab, et al. (1996). "Prediction of ASTM Sequence VI and VIA Fuel Economy Based on Laboratory Bench Tests." SAE 961140.

Gibbs, L. M. (1990). "Understanding Gasoline Additives." Automotive Engineering 98(1): 43-48.

Glidewell, J. and S. Korcek (1998). "Piston Ring / Cylinder Bore Friction Under Flooded and Starved Lubrication Using Fresh and Aged Engine Oils." SAE 982659.

Graham, J., H. Spikes, et al. (2001). "The Friction Reducing Properties of Molybdenum Dialklydithiocarbamate Additives: Part II - Durability of Friction Reducing Capability." Tribology Transactions 44(4): 637-647.

Graham, J., H. Spikes, et al. (2001). "The Friction Reducing Properties of Molybdenum Dialkyldithiocarbamate Additives: Part I - Factors Influencing Friction Reduction." Tribology Transactions 44(4): 626-636.

Grandin, H. M., B. Stadler, et al. (2006). "Waveguide Excitation Flourescence Microscopy: A New Tool for Sensing and Imaging at the Biointerface." Biosensors and Bioelectronics 21: 1476-1482.

Greenhill, E. B. (1949). "The Adsorption of Long Chain Polar Compounds from Solution on Metal Surfaces." Trans. Farad. Soc. 45: 625-635.

Gresham, R. M. (2003). Solid Film Lubricants -Unique Products For Unique Lubrication. Tribology and Lubrication Technology: 28-31.

grossiard, C., J. M. Martin, et al. (2000). "Tribochemical Interactions Between ZnDTP, MoDTC and Calcium Borate." Tribology Letters 8: 203-212.

Grossiard, C., K. Varlot, et al. (1998). "MoS2 Single Sheet Lubrication by Molybdenum Dithiocarbamate." Tribology International 31(12): 737-743. 
Gunsel, S. (1999). "The Elastohydrodynamic Friction and Film Forming Properties of Lubricant Base Oils." Tribology Transactions 42(3): 559-569.

Haluska, P. and L. Guzzella (1998). "Control Orientated Modeling of Mixture Formation Phenomena in Multi-Port Injection SI Gasoline Engines." SAE 980628.

Haury, E. J. (1996). "A Fleet Test Evaluation of the Effect of a Unique Gasoline Additive on Vehicle Acceleration." $\underline{\text { SAE } 962007 .}$

Hayden, T. E., C. A. Ropes, et al. (2001). "The Performance of a Gasoline Friction Modifier Fuel Additive." SAE 2001-01-1961.

Hoshino, K., H. Kawai, et al. (1998). "Fuel Efficiency of SAE 5W-20 Friction Modified Gasoline Engine Oil." SAE 982506.

Hsu, S. M. and R. S. Gates (2005). "Boundary Lubricating Films: Formation and Lubrication Mechanism." Tribology International 38: 305-312.

ILSAC (2004). "ILSAC GF-4 Standard for Passenger Car Engine Oils." www.ilma.org/resources/ilsac finalstd011404.pdf.

Jang, H. (1998). "The Effect of Solid Lubricants on Friction Characteristics." SAE 982235.

Jenkin, R. J., E. H. James, et al. (1996). "Thermal Boundary Layer Modelling in 'Motored' Spark Ignition Engines." SAE 961965.

Jensen, R. (2005). "Extending the Retention of Friction-Reducing Capability in Low-Phosphorus Engine Oils." Lubrication Science 17(2): 147-162.

Jost, P. (1966). "Lubrication (Tribology) Education and Research." $\underline{\text { HMSO. }}$

Kaiser, E., W. Seigl, et al. (1994). "Time-Resolved Measurement of Speciated Hydrocarbon Emissions During Cold Start of a Spark-Ignited Engine." SAE 940963.

Kajdas, C. (1993). Engine Oil Additives: a General Overview.

Kajdas, C., M. J. Furey, et al. (2002). "Triboemission as a Basic Part of the Boundary Friction Regime: A Review." Lubrication Science 14(2): 223-254. 
Kajdas, C. and K. Liu (2004). "Tribochemistry of Aluminium and Aluminium Alloy Systems Lubricated with Liquids Containing Alcohol or Amine Additive Types and Some Other Lubricants - A Review." Lubrication Science 16(3): 267-292.

Kano, M. (2006). "Super Low Friction of DLC Applied to Engine Cam Follower Lubricated With Ester Containing Oil." Tribology International 39(12): 1682-1685.

Kasrai, M., N. Cutler, et al. (1998). "The Chemistry of Antiwear Films Generated by the Combination of ZDDP and MoDTC Examined by X-ray Adsorption Spectroscopy." Tribology Transactions 41(1): 69-77.

Kenbeek, D., T. Buenemann, et al. (2000). "Review of Organic Friction Modifiers Contribution to Fuel Efficiency?" SAE 2000-01-1792.

Kikuchi, T., S. Ito, et al. (2003). "Piston Friction Analysis Using a Direct-Injection Single Cylinder Gasoline Engine." JSAE Review 24: 53-58.

Kikuchi, T., S. Ito, et al. (2003). "Piston Friction Analysus Using a Direct -Injection Single Cylinder Gasoline Engine." JSAE Review 24: 53-58.

Kim, H., S. Yoon, et al. (2003). "Correlating Port Fuel Injection to Wetted Fuel Footprints on Combustion Chamber Walls and UBHC in Engine Start Processes." SAE 2003-01-3240.

Korcek, S., R. Jensen, et al. (1999). "Fuel Efficient Engine Oils, Additive Interactions, Boundary Friction and Wear." Lubrication at the Frontier.

Korcek, S., R. K. Jensen, et al. (2000). "Interactions Leading to Formation of Low Friction Films in Systems Containing Molybdenum Dialkyldithiocarbamate and Zinc Dialkyldithiophosphate Additives." Thinning Films and Tribological Interfaces: 399 407.

Korcek, S. and M. Nakada (1996). "Engine Oil Performance Requirements and Reformulation for Future Gasoline Engines and Systems." SAE 961146.

Korcek, S., J. Sorab, et al. (2000). "Automotive Lubricants for the Next Millenium." Industrial Lubrication and Tribology 52(5): 209-220. 
Kovacs, A. (1995). "Visualisation of Fuel-Lubricant Interaction on the Cylinder Surface in the Combustion Chamber of SI Engines." Lubrication Science 7(2): 149162.

Leach, B., H. Zhao, et al. "Two-Phase Fuel Distribution in an Air Asisted DI Gasoline Engine."

Liu, K., Y. B. Xie, et al. (1998). "A comprehencive study of the friciton and dynamic motion of the piston assembly." Proceedings of the Institute of Mechanical Engineers 212(J): 221-226.

Lundgren, S. M., K. Persson, et al. (2006). "Adsorption of Fatty Acids from Alkane Solution Studied with Quartz Crystal Microbalance." Tribology Letters 22(1): 15-20.

Ma, M.-T. (1997). "Analysis of lubrication and friction for a complete piston-ring pack with an improved oil availability model. Part 1 : circumferential uniform film." Proceedings of the Institute of Mechanical Engineers 211(J): 1-15.

Ma, M.-T., I. Sherrington, et al. (1997). "Analysis of lubrication and friction for a complete piston-ring pack with improved oil availability model. Part II: Circumferentially variable film." Proceedings of the Institute of Mechanical Engineers 211(J): 17-27.

Manni, M., C. Gommellini, et al. (1995). "Effect of Physical Characteristics of Lubricating Oils on Emissions, Fuel Economu and Oil Consumption in a Light Duty Diesel Engine." $\underline{\text { SAE } 952552 .}$

Mansouri, S. H. and V. W. Wong (2004). "Effects of Piston Design Parameters on Piston Secondary motion and Skirt-Liner Friction." SAE 2004-01-2911.

Markley, K. S. (1960). Fatty Acids: Their Chemistry, Properties, Production and Uses, Interscience New York.

Markley, K. S. (1967). Fatty Acids: Thier Chemistry, Properties, Production and Uses, Interscience New York.

Marshall, W. F. (1979). "Potential for Improving Short-Trip Fuel Economy by Fuel Formulation." SAE 790655. 
Matsuo, K., S. Kiga, et al. (2005). "Reduction of Piston System Friction by Applying a Bore Circularity Machining Technique to the Cylinder Block." $\underline{\text { SAE 2005-01-1656. }}$

McCurry, J. D. and B. D. Quimby (2003). "Two-Dimensional Gas Chromatography Analysis of Components in Fuel and Fuel Additives Using a Simplified Heart Cutting GC System." Journal of Chromatographic Science 41: 524-527.

McGeehan, J. A. (1978). "A Literature Review of the Effects of Piston and Ring Friction and Lubricating Oil Viscosity on Fuel Economy." SAE 780673.

Metcalf, M. and J. Reid (1996). Fortran 90/95 Explained. Oxford, Oxford University Press.

Meyer, R. and J. B. Heywood (1997). "Liquid Fuel Transport Mechanisms into the Cylinder of a Firing Port-Injected SI Engine During Start Up." SAE 970865.

Miklozic, K. T., J. Graham, et al. (2001). "Chemical and Physical Analysis of Reaction Films Formed by Molybdenum Dialkl-Dithiocarbamate Friction Modifier Additive Using Raman and Atomic Force Microscopy." Tribology Letters 11(2): 7181.

Minfray, C., J. M. Martin, et al. (2003). "The Role of Mechanical and Chemical Processes in Anti-Wear Properties of ZDDP Tribofilms."

Mitchell, P. C. H. (1984). "Oil-Soluble Mo-S Compounds As Lubricant Additives.". Wear 100: 281-300.

Monaghan, M. L. (1989). "Putting Friction in its Place." Proceedings of the Institute of Mechanical Engineers C375/KN1.

Moreton, D. (2005). "Opportunities for Chemists in the Automotive Additive Industry." Lubrizol Internal Presentation.

Morina, A., J. H. Green, et al. (2003). "Surface and Tribological Characteristics of Tribofilms Formed in the Boundary Lubrication Regime with Application to Internal Combustion Engines." Tribology Letters 15(4): 443-452.

Mufti, R. (2004). Total and Component Friction in a Motored and Fired Engine. Mechanical Engineering. Leeds, The University of Leeds. PhD: 300. 
Mufti, R. and M. Priest (2005). "Experimental Evaluation of Piston Assembly Friction Under Motored and Fired Conditions in a Gasoline Engine." Jour. Tribology, ASME 127(4): 826-836.

Mufti, R., M. Priest, et al. (2004). Experimental and Theoretical Study of Instantaneous Piston Assembly Friction in a Gasoline Engine. 2002 ASME/STLE International Joint Tribology Conference, California.

Murakami, Y. and H. Aihara (1991). "Analysis of Mechanism Intermixing Combustion Products in Engine Oil (Quantity and Composition of Unbured Gasoline in Engine Oil and Crankcase Gas)." Jap. Soc. Mech. Engrs Int. J(Ser. II, 34(4)): 548-556.

Nakada, M. (1993). "Trends in Engine Technology and Tribology." Tribology International 27(1): 3-8.

Nakashima, K., S. Ishihara, et al. (1995). "Influence of Piston Ring Gaps on Lubricating Oil Flow Into The Combustion Chamber." SAE 952546.

Nakayama, K., S. Tamaki, et al. (2000). "The Effect of Crankshaft Offset on Piston

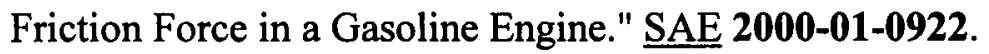

Nice, K. (2007). "How Stuff Works." www.auto.howstuffworks.com.

Nicholls, M. A., T. Do, et al. (2005). "Review of the Lubrication of Metallic Surfaces by Zinc Dialkyl-Dithiophosphates." Tribology International 38: 15-39.

Noorman, M. T., D. Assanis, et al. (2000). "Overview of Techniques for Measuring Friction Using Bench Tests and Fire Engines." SAE 2000-01-1780.

Norris, M. G. and S. Hochgreb (1994). "Novel Experiment on In-Cylinder Desorption of Fuel from the Oil Layer." SAE 941963.

O'Connor, D. J., B: A. Sexton, et al. (2003). Surface Analysis Methods in Materials Science, Springer.

OCTEL (2003). Octel Refinery Specialties. Octel.

OCTEL (2003). Performance Specialties. OCTEL. 
Oncins, G., J. Torrent-Burgues, et al. (2006). "Lateral Force Microscopy Study of Langmuid-Blodgett Films of a Macrocyclic Compound." Tribology Letters 21(3): 175-184.

Paul, A. J. (2005). Organic and Molecular Imaging by ToF-SIMS - At The Cutting Edge. Spectroscopy Europe. 17: 25-27.

Perfiliev, V., A. Moshokovith, et al. (2006). "A New Way to Feed Nanoparticles to Friction Interfaces." Tribology Letters 21(2): 89-93.

Ping, W. D., S. Korcek, et al. (1996). "Comparison of the Lubricity of Gasoline and Diesel Fuels." $\underline{\text { SAE }} 962010$.

Plint (1970). "TE-77 Handbook."

Priest, M. (1996). The Wear and Lubrication of Piston Rings. The Deparment of Mechanical Engineering. Leeds, The University of Leeds. PhD.

Priest, M. (2000). "Automobile Engine Tribology - Approaching the Surface." Wear 241 (2000): 193-203.

Priest, M. (2000). "Factors Influencing Boundary Friction and Wear of Piston Rings." Thinning Films and Tribological Interfaces: 409-416.

Priest, M. (2006). "Lubricants and Lubrication." Unit 3 MECH 3280, Principles of Tribology.

Pryde, E. H. (1985). Fatty Acids, The American Oil Chemists' Society.

Randolph, A. L. (1990). "Methods of Processing Cylinder-Pressure Transducer Signals to Maximise Data Accuracy." SAE 900170.

Reid, J. (2003). Introduction to Associated Octel + Performance Additives. Fuel Additives, Octel.

Reid, R. C., J. M. Prausnitz, et al. (1977). The Properties of Gases and Liquids. New York, McGraw-Hill.

Renondeau, H. (2005). "Science et Surface S. A." 
Richard, G. P. (1982). "Lubricant Properties in the Diesel Piston Ring Zone." $\underline{9}^{\text {th }}$ Leeds-Lyon Symposium on Tribology, Boddington Hall, The University of Leeds, England. Butterworths.

Robinson, I. C. H. (1977), 'The Effect of Gasoline Additives on Fuel Additive Fuel Economy', Fuel Economy of the Gasoline Engine, Blackmore, D. R., Thomas, A. ed. McMillan Press, Chapter 5, 77-88

Ruijun, Z., L. Shenghua, et al. (2006). "Tribochemical Interactions of Piston Ring Coated with Molybdenum Dithiocarbomate Friction Modifier." Journal of Materials Science 41(7): 1911-1915.

Russ, S., E. W. Kaiser, et al. (1995). "Effect of Cylinder Head and Engine Block Temperature on HC Emissions From a Single Cylinder Spark Ignition Engine." $\underline{\mathrm{SAE}}$ 952536.

Ryk, G. and I. Etison (2005). "Testing Piston Rings with Partial Laser Surface Texturing for Friction Reduction." Wear 261(7-8): 792-796.

Sanda, S., M. Murakami, et al. (1997). "Analysis of Lubrication of a Piston Ring Package - Effect of Oil Starvation on Oil Film Thickness." JSME International Journal Series B, 40(3): 478-486.

Sarin, R., D. K. Tuli, et al. (1994). "Additive-Additive Interactions; Search for Synergistic FM-EP-AW Composition." WEAR 174: 93-102.

Saville, S. B., F. D. Gainey, et al. (1988). "A Study of Lubricant Condition in the Piston Ring Zone of Single-Cylinder Diesel Engines Under Typical Operating Conditions." $\underline{\text { SAE } 881586 .}$

Schramm, J. and S. C. Sorenson (1991). "Solubility of Gasoline Components in Different Lubricants for Combustion Engines Determined by Gas-Liquid Partition Chromatography." journal of Chromatography 538: 241-248.

Schulz, C. and V. Sick (2005). "Tracer-LIF Diagnostics: Quantitative Measurement of Fuel Concentration, Temperature and Fuel/Air Ratio in Practical Combustion Systems." Progress in Energy and Combustion Science 31: 75-121. 
Shaub, H., J. Pandosh, et al. (1994). "Engine Durability, Emissions and Fuel Economy Studies with Special Boundary Lubricant Chemistry." SAE 941983.

Shin, Y., W. K. Cheng, et al. (1994). "Liquid Gasoline Behaviour in the Engine Cylinder of a SI Engine." SAE 941872.

Skippon, S. M. and Y. Tagaki (1996). "ILIDS Measurement of the Evaporation of Fuel Droplets During the Intake and Compression Strokes in a Firing Lean Burn Engine." SAE 960830.

Smith, G. (2002). "Intimate Contact." Shell Internal.

Smith, G. C. (1994). Surface Analysis by Electron Spectroscopy: Measurement and Interpretation, Plenum Press, New York.

Smith, O. M. Priest, et al (2006). "Simulated Fuel Dilution and Friction-Modifier Effects on Piston Ring Friction." Proceedings of the Institute of Mechanical Engineers 220(J), $3: 181-189$.

Sorab, J., S. Korcek, et al. (1998). "Friction Reduction in Lubricated Components Through Engine Oil Formulation." SAE 982640.

Spikes, H. (1971). Physical and Chemical Adsorption in Boundary Lubrication. Mechanical Engineering. London, Imperial College. PhD.

Spikes, H. (1999). "Thin Films In Elastohydrodynamic Lubrication: The Contribution of Experiment." Proceedings of the Institute of Mechanical Engineers 213(J): 335-352.

Spikes, H. (2002). "Film-Forming Additives - Direct and Indirect Ways to Reduce Friction." Lubrication Science 14(2): 147-167.

Spikes, H. (2004). "The History and Mechanisms of ZDDP." Tribology Letters 17(3): 469-489.

Spikes, H. and A. V. Olver (2003). "Basics of Mixed Lubrication." Lubrication Science 16(1): 3-28. 
Srivastava, R., C. Bao, et al. (1996). "Planar-Surface-Waveguide Evanescent-Wave Chemical Sensors." Sensors and Actuators 51: 165-171.

Subaru (2006). "www.drivesubaru.com."

Sunqing, Q., D. Junxiu, et al. (1999). "Tribological Properties of CeF3 Nanoparticles as Additives in Lubricating Oils." Wear 230: 35-38.

Swindal, J. C., D. P. Dragonetti, et al. (1995). "In-Cylinder Charge Homogeneity During Cold-Start Studied With Flourescent Tracers Simulating Different Fuel Distillation Temperatures." SAE 950106.

Swindal, J. C., P. A. Furman, et al. (1997). "Fuel Distillation Effects on the Outgassing from a Simulated Crevice in a SI Engine Measured by PLanar LaserInduced Flourescence." $\underline{\text { SAE } 970825 .}$

Tan, Y., W. Huang, et al. (2002). "Molecular Orbital Indexes Criteria For Friction Modifiers In Boundary Lubrication." Tribology International 35: 381-384.

Taylor, C. M. (1998). "Automobile Engine Tribology - Design Considerations for Efficiency and Durability." WEAR 221 (1998) 1-8.

Taylor, I. (2002). Car Lubricants: Fact and Friction. Physics World: 39-43.

Taylor, R. I. (1994). "Lubricants and Lubrication - Validation of a piston ring-pack lubrication model that includes realistic lubricant rheology." Tribology Series 30(21st Leeds Lyon Symposium): 345-354.

Taylor, R. I. (1997). "Engine Friction: The Influence of Lubricant Rheology." Proceedings of the Institute of Mechanical Engineers 211(J): 235-246.

Taylor, R. I. (1997). Engine Friction: the influence of lubricant rheology (2). Fifth CEC International Symposium on the Performance Evaluation of Automotive Fuels and Lubricants, Goteborg, Sweden.

Taylor, R. I. (1998). "The Inclusion of Lubricant Shear Thinning in the Short Bearing Approximation." Proceedings of the Institute of Mechanical Engineers 213(Part J): 35-46. 
Taylor, R. I. (1999). "Engine Friction Lubricant Sensitivities: A Comparison of Modern Diesel and Gasoline Engines." Shell Internal.

Taylor, R. I. (2000). "Heavy Duty Diesel Fuel Economy: Lubricant Sensitivities." SAE 2000-01-2056.

Taylor, R. I. (2002). The Development of Fuel Economy Lubricants. Fuels and Lubes Asia Conference and Exhibition, Singapore.

Taylor, R. I. (2002). "Lubrication, Tribology and Motorsport." 2002-01-3355 SAE.

Taylor, R. I., M. A. Brown, et al. (1995). "Validation of a Piston Ring-Pack Lubrication Model that Includes Realistic Lubricant Rheology." Lubricants and Lubrication 30.

Taylor, R. I., M. A. Brown, et al. (1994). "The Influence of Lubricant Rheology on Friction in the Piston Ring-Pack." SAE 941981.

Taylor, R. I. and R. C. Coy (2000). "Improved Fuel Effeciency by Lubricant Design: a Review." Proceedings of the Institute of Mechanical Engineers 214(J): 1-15.

Taylor, R. I. and P. G. Evans (2004). "In-situ Piston Measurement." Proceedings of the Institute of Mechanical Engineers 218(J): 185-200.

Taylor, R. I., T. Kitahara, et al. (1995). Piston Assembly Friction and Wear: The Influence of Lubricant Viscometry. Proceedings of the International Tribology Conference, Yokahama.

Taylor, R. I., R. Mainwaring, et al. (2005). "Engine Lubricant Trends Since 1990." Proceedings of the Institute of Mechanical Engineers 219(J): 331-345.

Taylor, R. I., N. R. Nagatomi, et al. (2000). "A Screener Test for the Fuel Economy Potential of Engine Lubricants." Shell Internal.

Theil, Y. (2001a). Friction Modifier Delivered to Engine Oil Via Fuel: Predicting and monitoring a Performance Favourable Effect. Asia Fuels and Lubricants Conference. 
Thiel, Y. C. and T. E. Hayden (2001b). "The Fuel Additive / Lubricant Interactions:

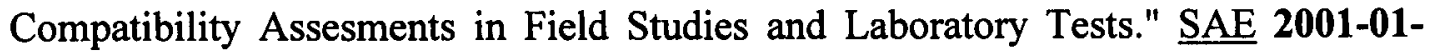
1962.

Thompson, A. (1996). The Tribological Performance of Piston Ring Packs Designed for Low Emissions. Mechanical Engineering. Leeds, The University of Leeds: 218.

Thompson, A., C. D. Radcliffe, et al. (1997). "The Influence of Piston Ring Tribology on the Diffusion of Fuel Through Oil Films." Proceedings of the Institute of Mechanical Engineers 211(J): 223-233.

Thompson, G. P. and W. Cochrane (1939). Theory and Practice of Electron Diffraction, Macmillan.

Thompson, M. (1990). "The Use of Top Ring Zone Sampling and Analysis to Investigate Oil Consumption Mechanisms." IMechE c394/041.

Thring, R. H. (1992). "Engine Friction Modelling." SAE 920482.

Tian, T., V. Wong, et al. (1998). "Modeling the Dynamics and Lubrication of Three Piece Oil Control Rings in Internal Combustion Engines." SAE 982657.

Transportation, U. S. D. o. (2004). Summary of Fuel Economy Performance. J. Finnerman. Washington DC.

Tripaldi, G., V. Vettor, et al. (1996). "Friction Behaviou of ZDDP Films in the Mixed, Boundary / EHD Regime." $\underline{\text { SAE } 962036 .}$

Truhan, J. J., J. Qu, et al. (2005). "A Rig Test to Measure Friction and Wear of Heavy Duty Diesel Engine Piston Rings and Cylinder Liners Using Realistic Lubricants." Tribology International 38: 211-218.

Tseregounis, S. I. and M. L. McMillan (2001). "Fuel Economy Gains with Modern Technology, SAE 5W-20 Engine Oils in a GM Engine as Measured in the EPA FTP Test." $\underline{\text { AAE 2001-01-1900. }}$

Tseregounis, S. I., M. L. Mcmillan, et al. (1998). "Engine Oil Effects on Fuel Economy in GM Vehicles - Separation of Viscosity and Friction Modifier Effects." SAE 982502. 
Tung, S. C. and H. Gao (2003). "Tribological Characteristics and Surface Interaction Between Piston Ring Coatings and a Blend of Energy-Conserving Oils and Ethanol Fuels." Wear 255: 1276-1285.

Tung, S. C. and S. I. Tseregounis (2000). "An Investigation of Tribological Characteristics of Energy-Conserving Engine OIls Using A REciprocating Bench Test." SAE 2000-01-1781.

Tupa, R. C. and C. J. Dorer (1986). "Gasoline and Diesel Fuel Additives for Performance/Distribution Quality - II." SAE 861179.

Unnikrishnan, R., M. C. Jain, et al. (2002). "Additive-Additive Interaction: an XPS Study of the Effect of ZDDP on the AW/EP Characteristics of Molybdenum Based Additives." Wear 252: 240-249.

Vettor, V., G. Tripaldi, et al. (2003). "Mo-Compounds Efficiency and Interactions

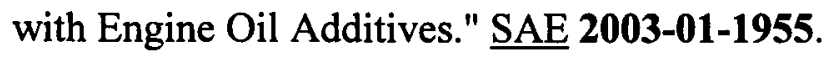

Vickerman, J. C. (1997). Surface Analysis - The Principle Techniques, John Wiley and Sons, Chichester.

Vipper, A. B., W. Bartz, et al. (1995). "Antifriction Action of Lubricant Additives." Lubrication Science 7(3): 247-259.

Vipper, A. B., O. P. Parenago, et al. (1999). "Tribological Performance of Molybdenum and Zinc Dithiocarbamates and Dithiophosphates." Lubrication Science 11(2): 187-196.

Wain, K. S., J. M. Perez, et al. (2005). "Alternative and Low Sulfur Fuel Options: Boundary Lubrication Performance and Potential Problems." Tribology International 38: 313-319.

Wakabayashi, R., M. Takiguchi, et al. (2003). "The Effects of Crank Ratio and Crankshaft Offset on Piston Friction Losses." SAE 2003-01-0983.

Wakuri, Y., T. Hamatake, et al. (1992). "Piston Ring Friction in Internal Combustion Engines." Tribology International 25(5): 299-308. 
Wakuri, Y., M. Soejima, et al. (1995). "Studies on Friction Characteristics of Reciprocating Engines." SAE 952471.

Wanli, Y., Y. Chen, et al. (2004). "Simulation of Non-Steady Multidimensional Heat Transfer Within Piston / Cylinder Liner Sliding Couple of Internal Combustion Engine." SAE 2004-01-0599.

Wedler, G. (1970). Chemisorption: An Experimental Approach, The Butterworth Group.

Wei, D. P., H. Spikes, et al. (1999). "The Lubricity of Gasoline." Tribology Transactions 42(4): 813-823.

Wilk, M. A. and M. S. Newkirk (1998). "Toward Improved Fuel Economy in Passenger Car Motor Oils: An Investigation into the Influence of Detergent System and Friction Modifier as Measured by the EPA Federal Test Procedure and Highway Fuel Economy Test Cycles." SAE 982505.

Willcock, M. (1996). Recent Developments in Piston Assemblies.

Williams, J. A. (1994). Engineering Tribology, Oxford University Press.

Wilson, B. (1995). "Slick 50- A Proven PTFE-Based Boundary Lubricant For Engines." Industrial Lubrication and Tribology 47(4): 6-8.

Winborn, L. D. and P. J. Shayler (2001). "Fuel Losses to the Crankcase and Hydrocarbon Return with Recirculated Oil and Ventilation Flow." Proceedings of the Institute of Mechanical Engineers 215(10): 1117-1130.

Yamada, Y., M. Ishimaru, et al. (2000). "Retention of Friction Reducing Performance of MoDTC-Containing Fuel Efficient Gasoline Engine Oils During Use." $\underline{\text { SAE 2000-01-2053. }}$

Yamada, Y., M. Ishimaru, et al. (2001). "Retention of Friction Reducing Performance of MoDTC-Containing Fuel Efficient Gasoline Engine Oils During Use." $\underline{\text { SAE 2001-01-2053. }}$

Yamaguchi, E. S., S. H. Roby, et al. (2005). "Time-Dependent Film Formation from ZnDTPs and Nonphosphorus Antiwear Agents." Tribology Transactions 48: 57-68. 
Zaslavskii, Y. S., R. N. Zaslavskii, et al. (1973). "Possible Future Developments in the use of Substances Forming Friction Polymers." UDC 665: 716-719.

Zhou, Q., M. C. Lai, et al. (1995). "The Spray Characteristics of Automotive Port Fuel Injection - A Critical Review." SAE 950506.

Zhou, Q., I. Shilling, et al. (2003). "Prediction of total engine friction power loss from detailed component models." Tribological Research and Designs for Engineering Systems: 761-766.

Zughyer, J. R., F. C. Zhao, et al. (2000). "A Visualization Study of Liquid Fuel Distribution and Combustion Inside a Port-Injected Gasoline Engine Under Different Start Conditions." SAE 2000-01-0242. 


\section{Appendix}

\section{A1: Determination of Normal Force Present At the Cylinder Liner / Piston Ring Interface:}

When determining the normal force required in order to accurately simulate the cylinder liner / piston ring interface conditions, it is necessary to have a basic knowledge of operating engine conditions. It is known, therefore, that the maximum cylinder pressure at medium speed conditions, present a few degrees after TDC, is typically in the region of $4 \mathrm{MPa}$. The following table represents the operating conditions as related to maximum cylinder pressure:

\begin{tabular}{ccc}
\hline Engine Speed (rpm) & BMEP (bar) & $\begin{array}{l}\text { Maximum Cylinder } \\
\text { Pressure (MPa) }\end{array}$ \\
\hline 2500 & 5 & 4.0 \\
\hline
\end{tabular}

Typical Hydra Gasoline Engine Data (Gamble 2002a)

For the purpose of this study, it is proposed that the maximum cylinder pressure of $4 \mathrm{MPa}$ should be employed in order to simulate a worst case scenario.

It is assumed that the force created within the cylinder, and subsequently acting behind the piston ring, is significantly greater than any other force acting at the interface. Other forces can consequently be ignored.

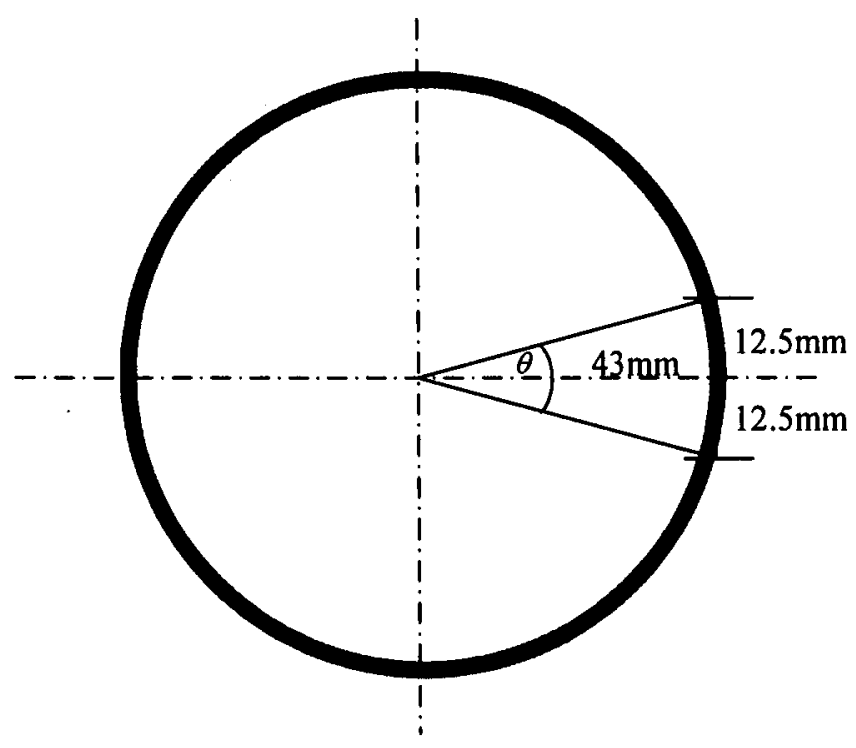




\section{Force Calculation:}

The angle $\theta$, representing half the arc angle $=\sin ^{-1}\left[\frac{12.5}{43}\right]=0.29 \mathrm{rad}$

Therefore, $2 \theta=0.59 \mathrm{rad}$

The fraction of the circumference this represents: $\frac{0.59}{2 \pi}=0.094 \mathrm{rad}$

$$
\text { Hence, length of } \operatorname{arc}=(86 \pi) \bullet 0.094=25.37 \mathrm{~mm}
$$

The area over which the pressure acts, $\mathrm{A}=\operatorname{arc} \mathrm{x}$ Width of Piston Ring

$$
\mathrm{A}=1.5 \times 10^{-3} \cdot 25.37 \times 10^{-3}=3.8 \times 10^{-5} \mathrm{~m}^{2}
$$

Maximum cylinder pressure occurring in the Hydra engine $\mathrm{P}_{\max }=4 \mathrm{MPa}$

$$
\mathrm{P}_{\max }=\frac{F}{A}
$$

Therefore, $F=152 \mathrm{~N}$ 


\section{A2: Shell XHVI-8.2 Datasheet}

\section{Shell XHVI 8.2}

Extra high viscosity index base oil

Shell XHVI (Extra High Viscosity Index) base oils are special products from the Shell XHVI synthesis process. With the VI above 120 they are classified as APIIATIEL

Group III type base oil. XHVI base oils are favourable blending components for high quality speciality lubricants in the automotive and industrial sector, where excellent viscosity-temperature behaviour and low volatility are required.

Typical Physical Characteristics

\begin{tabular}{|c|c|c|c|c|}
\hline & & & XHVI 8.2 & $\begin{array}{c}\text { XHVI } 8.2 \\
\text { Specification }\end{array}$ \\
\hline Appearance & & & bright\&clear & \multirow{3}{*}{$\begin{array}{c}\text { bright\&clear } \\
\max 1.0\end{array}$} \\
\hline Colour (ASTM) & & ASTM D 1500 & Lo.5 & \\
\hline Density at $15^{\circ} \mathrm{C}$ & $\mathrm{kg} / \mathrm{m} 3$ & ASTM D 1298 & 834 & \\
\hline Flashpoint PMCC & ${ }^{\circ} \mathrm{C}$ & ASTM D 93 & 240 & \multirow[t]{2}{*}{$\min 210$} \\
\hline Flashpoint COC & ${ }^{\circ} \mathrm{C}$ & ASTM D 92 & 250 & \\
\hline Pour Point & ${ }^{\circ} \mathrm{C}$ & ASTM D 97 & -15 & \multirow[t]{2}{*}{$\max -15$} \\
\hline KInematlc Viscosity at $40^{\circ} \mathrm{C}$ & $\mathrm{mm} 2 / \mathrm{s}$ & ASTM D 445 & 48 & \\
\hline Kinematic Viscosity at $100^{\circ} \mathrm{C}$ & $\mathrm{mm} 2 / \mathrm{s}$ & ASTM D 445 & 8.3 & \multirow{5}{*}{$\begin{array}{l}7.8 \cdot 8.5 \\
\min 140 \\
\max 0.05\end{array}$} \\
\hline Viscosity Index & & ASTM D 2270 & 147 & \\
\hline Acld Value & $\mathrm{mgKOH} / \mathrm{g}$ & ASTM D 974 & $<0.03$ & \\
\hline Ash & $\% \mathrm{~m}$ & ASTM D 482 & $<0.01$ & \\
\hline Sulphur Content & $\% \mathrm{~m} / \mathrm{m}$ & ASTM D 2622 & $<0.03$ & \\
\hline Noack Volatillity & $\% m$ & ASTM D 5800 & 5 & $\max 5$ \\
\hline
\end{tabular}

These characteristics are typical of current production. Whilst future production will conform

to Shell's specification, veriations in these characteristics may occur. 


\section{A3: Shell XHVI-4TM Datasheet}

\section{Shell XHVI 4.0}

Extra high viscosity index base oil

Shell XHVI (Extra High Viscosity Index) base oils are special products from the Shell XHVI synthesis process. With the VI above 120 they are classifled as API/ATIEL

Group III type base oll. XHVI base oils are favourable blending components for high quality speciality lubricants in the automotive and industrial sector, where excellent viscosity-temperature behaviour and low volatility are required.

\section{Typical Physical Characteristics}

\begin{tabular}{|c|c|c|c|c|}
\hline & & & XHVI 4.0 & $\begin{array}{c}\text { XHVI } 4.0 \\
\text { Specification }\end{array}$ \\
\hline Appearance & & & bright\&clear & \multirow{3}{*}{$\begin{array}{c}\text { bright\&clear } \\
\max 1.0\end{array}$} \\
\hline Colour (ASTM) & & ASTM D 1500 & L0.5 & \\
\hline Density at $15^{\circ} \mathrm{C}$ & $\mathrm{kg} / \mathrm{m3}$ & ASTM D 1298 & 819 & \\
\hline Flashpoint PMCC & ${ }^{\circ} \mathrm{C}$ & ASTM D 93 & 215 & \multirow[t]{2}{*}{$\min 210$} \\
\hline Flashpoint COC & ${ }^{\circ} \mathrm{C}$ & ASTM D 92 & 220 & \\
\hline Pour Point & ${ }^{\circ} \mathrm{C}$ & ASTM D 97 & -18 & \multirow[t]{2}{*}{$\max -18$} \\
\hline Kinematic Viscosity at $40^{\circ} \mathrm{C}$ & $\mathrm{mm} 2 / \mathrm{s}$ & ASTM D 445 & 17 & \\
\hline KInematic Viscosity at $100^{\circ} \mathrm{C}$ & $\mathrm{mm} 2 / \mathrm{s}$ & ASTM D 445 & 4.0 & \multirow{3}{*}{$\begin{array}{l}3.8-4.2 \\
\min 140 \\
\max 0.05\end{array}$} \\
\hline Viscosity Index & & ASTM D 2270 & 141 & \\
\hline Acid Value & $\mathrm{mgKOH} / \mathrm{g}$ & ASTM D 974 & $<0.03$ & \\
\hline Ash & $\% m$ & ASTM D 482 & $<0.01$ & \multirow[b]{3}{*}{$\max 15-17\left(^{*}\right)$} \\
\hline Sulphur Content & $\% \mathrm{~m} / \mathrm{m}$ & ASTM D 2622 & $<0.03$ & \\
\hline Noack Volatility & $\% \mathrm{~m}$ & ASTM D 5800 & 15 & \\
\hline
\end{tabular}

These characteristics are typical of current production. Whilst future production will conform to Shell's specification, variations in these characteristics may occur. 


\section{A4: Gasoline Specifications}

\begin{tabular}{|c|c|c|c|c|c|}
\hline Base fuel & $\begin{array}{l}\text { Additive } \\
\text { package }\end{array}$ & Volume & Potassium & $\begin{array}{l}\text { Name for } \\
\text { Leeds }\end{array}$ & ChIP I.D. \\
\hline $\begin{array}{l}\text { SPL1995/03 } \\
\text { (STD) }\end{array}$ & $\begin{array}{ll}\text { 275ppmw } & \text { of } \\
\text { ADX 766L } & \end{array}$ & $200 \mathrm{~L}$ & $10 \mathrm{mg} / \mathrm{kg}$ & Fuel Aa & TBR9021/03 \\
\hline $\begin{array}{l}\text { SPL1995/03 } \\
\text { (STD) }\end{array}$ & $\begin{array}{ll}550 p p m w & \text { of } \\
\text { ADX 766L } & \end{array}$ & $200 \mathrm{~L}$ & $21 \mathrm{mg} / \mathrm{kg}$ & Fuel $\mathrm{Ab}$ & TBR9023/03 \\
\hline $\begin{array}{l}\text { SPL1995/03 } \\
\text { (STD) }\end{array}$ & $\begin{array}{l}\text { 1100ppmw of } \\
\text { ADX 766L }\end{array}$ & $200 \mathrm{~L}$ & $42 \mathrm{mg} / \mathrm{kg}$ & Fuel Ac & TBR9024/03 \\
\hline $\begin{array}{l}\text { TBR8647/03 } \\
\text { (HBP) }\end{array}$ & $\begin{array}{ll}\text { 275ppmw } & \text { of } \\
\text { ADX 766L }\end{array}$ & $200 \mathrm{~L}$ & $10 \mathrm{mg} / \mathrm{kg}$ & Fuel $\mathrm{Ba}$ & TBR9025/03 \\
\hline
\end{tabular}

\begin{tabular}{|c|c|c|c|}
\hline Base Fuel & & TBR8647/03 & SPL1995/03 \\
\hline Density@15 & $\mathrm{Kg} / \mathrm{L}$ & 0.7748 & 0.7243 \\
\hline$\overline{R V P}$ & mbar & 618 & 574 \\
\hline MON & & 87 & 87.6 \\
\hline \multicolumn{4}{|l|}{ DISTILLATION } \\
\hline IBP & ${ }^{\circ} \mathrm{C}$ & 32.1 & 33.1 \\
\hline FBP & ${ }^{\circ} \mathrm{C}$ & 203.8 & 168.8 \\
\hline E70 & $\% \mathrm{v}$ & 14.1 & 27.9 \\
\hline E100 & $\% \mathrm{v}$ & 29.6 & 48.7 \\
\hline E120 & $\% \mathrm{v}$ & 50.1 & 72 \\
\hline E150 & $\% \mathrm{v}$ & 80.9 & 94.6 \\
\hline Carbon & & 7.08 & 6.77 \\
\hline Hydrogen & & 11.85 & 13.48 \\
\hline Oxygen & & 0 & 0 \\
\hline AFR(stoichiometric) & & 14.28 & 14.74 \\
\hline Enthalpy Comb MJ/Kg (Gas) & & -42.815 & -43.903 \\
\hline Enthalpy Comb MJ/Kg (liq.) & & -42.439 & -43.546 \\
\hline \multirow[t]{3}{*}{ Calculated } & $\mathrm{H} / \mathrm{C}$ & 1.67373 & 1.99114 \\
\hline & $\mathrm{O} / \mathrm{C}$ & 0.00000 & 0.00000 \\
\hline & CWF & 0.87684 & 0.85682 \\
\hline Sulphur & $\mathrm{mg} / \mathrm{Kg}$ & 100 & 12 \\
\hline Unwashed gum & $\mathrm{mg} / 100 \mathrm{ml}$ & 2 & 4 \\
\hline Washed gum & $\mathrm{mg} / 100 \mathrm{ml}$ & 1 & $<1$ \\
\hline
\end{tabular}


A5: TPS System Manufacturing Information
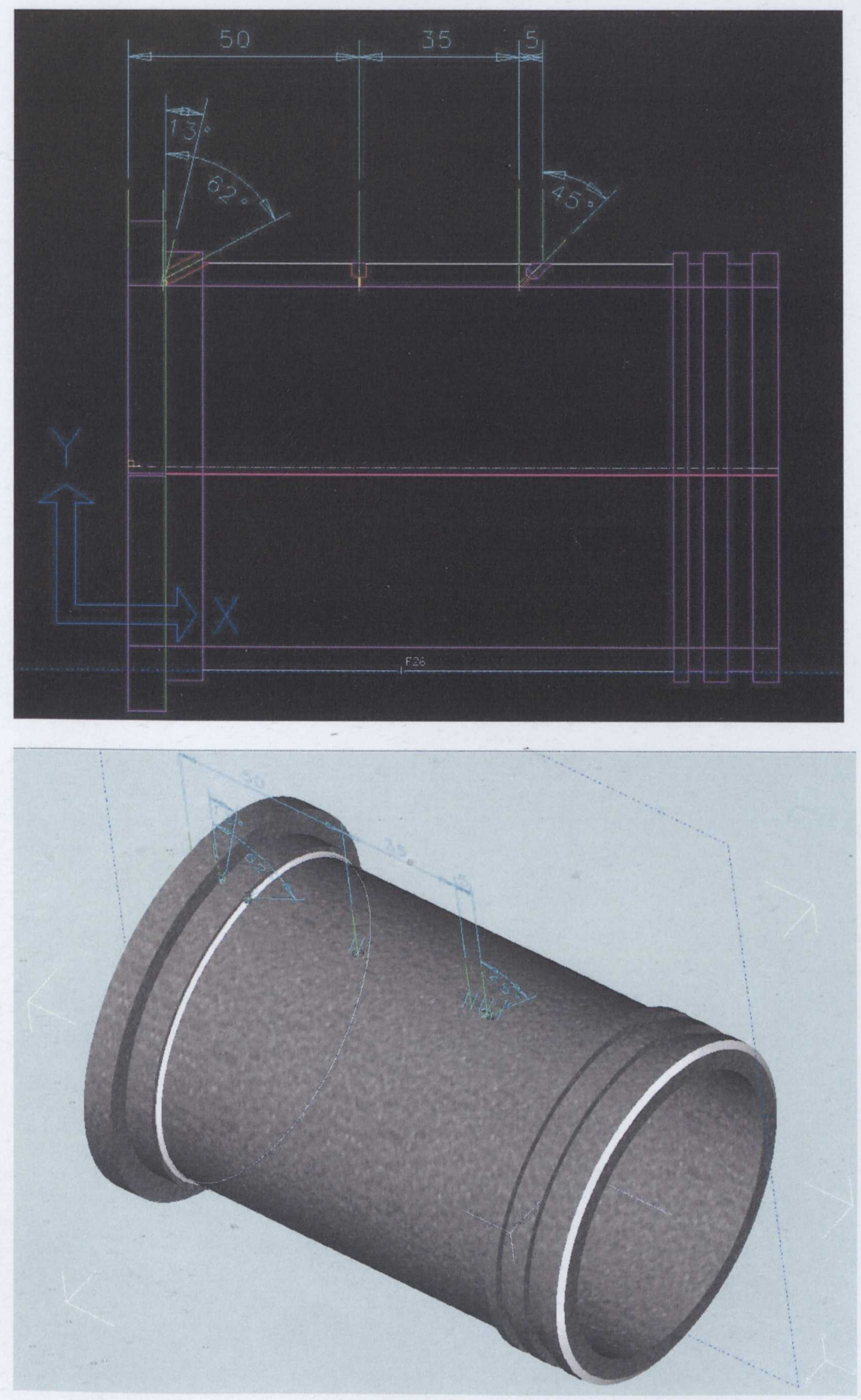